Jỗo de Sá Brasil Lima

\title{
CÁlculo de Sensibilidades \\ Geométricas E NÃo-GEOMÉTRICAS PARA ESCOAMENTOS VISCOSOS INCOMPRESSÍVEIS UTIlizAndo o Método Adjunto
}

Tese apresentada à Escola Politécnica da Universidade de São Paulo para obtenção do Título de Doutor em Ciências. 
Jỗo de Sá Brasil Lima

\title{
CÁlculo de Sensibilidades \\ Geométricas E NÃo-GeométriCAs PARA ESCOAMENTOS VISCOSOS INCOMPRESSÍVEIS UTILIZANDo o MÉtodo Adjunto
}

\author{
Tese apresentada à Escola Politécnica \\ da Universidade de São Paulo para ob- \\ tenção do Título de Doutor em Ciências. \\ Área de concentração: \\ Engenharia Mecânica \\ Orientador: \\ Prof. Dr. Ernani Vitillo Volpe
}


Este exemplar fol revisado e corrigido em relaçăo a versta original, sob responsabilidade única do autor e com a anuencla de seu orlentador.

sto Paulo, de de

Assinatura do autor.

Assinatura do orlentador.

\section{Catalogação-na-publicação}

Brasil Lima, Joäo

Cálculo de Sensibilidades Geométricas e Näo-Geométricas para Escoamentos Viscosos Incompressiveis Utilizando o Método Adjunto / J. Brasil Lima - versăo corr. - Săo Paulo, 2017. $130 \mathrm{p}$.

Tese (Doutorado) - Escola Politécnica da Universidade de Säo Paulo. Departamento de Engenharia Mecárica.

1.Otimizaçäo 2.Método Adjunto 3.CFD 4.Escoamento Incompressivel I.Universidade de Säo Paulo. Escola Politécnica. Departamento de Engenharia Mecânica Il.t. 


\section{DeDicatóRIA}

A meus pais, Guacyra e Celso

À minha namorada, Juliana

À minha avó, Maria

À memória das minhas avós, Imaculada e Vera, e de meus avôs Celso e João 


\section{Agradecimentos}

Não é clichê algum falar que sozinho a realização deste trabalho não seria possível. Portanto vou me estender um pouco nos agradecimentos pois não poderia me furtar de deixar esse singelo "muito obrigado"a todos que menciono aqui.

No âmbito acadêmico, agradeço inicialmente ao meu orientador, Prof. Dr. Ernani Vitillo Volpe, por ter literalmente me orientado durante todo esse tempo em que trabalhamos juntos desde o final da minha graduação. Agradeço por ter dividido comigo todas as emoções e etapas pertinentes a um doutorado: as descobertas, conquistas, dúvidas, dificuldades e angústias. Agradeço também por ter se envolvido em todos os momentos do meu trabalho e por confiar em mim em todos esses anos. E não posso deixar de agradecer também por todos os churrascos de comemoração (ou não) de alguma etapa do nosso grupo de pesquisa. Que venham muitos outros!

Ao Prof. Dr. Bruno Souza Carmo pelas valiosas contribuições ao longo deste trabalho sobretudo no exame de qualificação e pela oportunidade em trabalhar com ele em disciplina de graduação.

Ao Prof. Dr. Rafael dos Santos Gioria, pelas incalculáveis contribuições, sugestões e todos os "socorros"durante este trabalho. Com certeza sem suas contribuições muito do pouco que aqui foi conquistado não seria possível.

Aos demais professores do NDF, representados pelo coordenador do laborátorio Prof. Dr. Julio Meneghini, pela excelente infra-estrutura de trabalho que me foi proporcionada. Agradecimentos extensivos à Lúcia Messa, secretária mais eficiente que um laboratório pode ter, por todos os "galhos"quebrados e problemas resolvidos.

À Profa. Dra. Cristina Cerri do IME-USP por todas as oportunidades que me foram dadas de trabalho em disciplinas de graduação desde 2009 e por sempre confiar no meu trabalho.

Ao Dr. Xuerui Mao, da Universidade de Nottingham, pelas contribuições a respeito do código computacional e por ter me recebido na Universidade de Durham no ano de 2013. 
Ao Prof. Dr. Joseph Saab, pela amizade que se iniciou durante o cumprimento dos créditos e por toda a colaboração entre nós desde então.

Às secretárias da Pós Graduação, Marisa e Regiane, pela prestatividade, competência e disposição em ajudar em todos os momentos (mesmo nos menos convenientes) sempre com bom humor e simpatia

Ao "sócio"e doutorando Bruno Chieregatti, pela amizade que nasceu de forma inesperada, nos uniu como amigos e parceiros de pesquisa e de diversos trabalhos e por toda a ajuda durante o meu doutorado. Ao agora Prof. Dr. Marcelo Hayashi que, juntamente com o Bruno, contribiu para o desenvolvimento deste trabalho. E ao mestre Jairo Cavalcanti, recém integrado ao grupo de pesquisa. E obrigado a os três por todos os diálogos não acadêmicos que tornaram os dias mais leves e divertidos.

Ao matemático mais engenheiro que existe, Tomás Privato, por toda a colaboração dada com sugestões, simulações, brainstorms e tudo mais que ele se dispôs a fazer.

Aos demais colegas de NDF, João Anderson, Murilo Ciccolin e Gustavo Patino pelos diálogos nos corredores ligados à pesquisa ou não (maioria dos casos).

À CAPES pelo apoio financeiro durante todo o doutorado, à FAPESP pelo auxílio para ida a um Congresso e à FUSP pelo apoio financeiro dado durante o tempo em que trabalhei no RCGI concomitantemente ao doutorado.

À Escola Politécnica da USP e à Universidade de São Paulo pela oportunidade de estudar na melhor universidade do país desde 2007.

Nos âmbitos pessoal e familiar, começo agradecendo aos três amores da minha vida: meus pais e minha namorada.

Aos meus pais, Guacyra e Celso, por todo o amor, incentivo e por me apoiarem em todos os meus projetos e decisões da minha vida. E obrigado por terem sempre colocado a minha educação em primeiro lugar, muitas vezes abrindo mão de muito para que eu pudesse ter um estudo de qualidade. Espero honrar esse sacrifício de vocês por toda a minha vida. Amo vocês!

À minha namorada, amiga e companheira de todas as horas, Juliana, por todo o amor, paciência e compreensão não só durante este trabalho mas durante todo o tempo em que estamos juntos. Agradeço por ter me incentivado quando decidi ingressar na pós-graduação e por ter me apoiado sempre que o ânimo caiu. Como diria nosso "lema": Sempre juntos. Amo você! 
Aos meus familiares: minhas tias Gláucia e Maria Angélica; meu tio Nelson; meus primos Pedro, Maria Rita, Maurício e Rodrigo e à minha avó Maria Aparecida.

À minha madrinha Cristina, não só por todo o carinho mas também por ter me dado a honra de ter sido seu aluno um dia.

Aos meus amigos dos tempos de escola e cursinho: Leandro, Fernando, Thiago, Bárbara, Fernando Azevedo, Giancarlo Áquila, Bruno Kim e João Carlos. Aos amigos da Poli: Caio Santos e Renato Orsino. Todos os momentos com vocês foram e são sempre especiais e ajudaram a manter o ânimo aceso para ir até o fim desta etapa.

A todos os amigos do CEAE Perdizes, os quais não citarei nomes para não acabar esquecendo de alguém injustamente.

Aos amigos e sócios Benildo e Ricardo, pela amizade, momentos de descontração e pela compreensão nos meus momentos de ausência.

Aos amigos do Palmeiras, em especial ao Paulo Estêvão, Tito Maule, Renato Tessare e Edmar Zanatta.

Por fim gostaria de agradecer a todos os professores de quem tive a honra de ser aluno. Cada um me marcou de uma determinda forma e contribuiu para a minha formação e escolha em cumprir esta etapa. Do Colégio Pentágono, agradeço aos Profs. Américo Santos, Yuri Sano, Marcly Castro, Evaristo Martins e Marilice Toledo. Do Colégio Rio Branco, agradeço aos Profs. Ulisses Andreis, Pedro D’incao, Henrique Araujo, Maria Clara Leite, Roberto Fiori, Silvio Higa, João Jonas e Ocimar Morais.

Se tiver esquecido algúem deixo aqui meu pedido de desculpas pois são muitas pessoas para lembrar o que me deixa ainda mais feliz por ter, em algum momento, tido contato e contado com tante gente. Muito obrigado! 

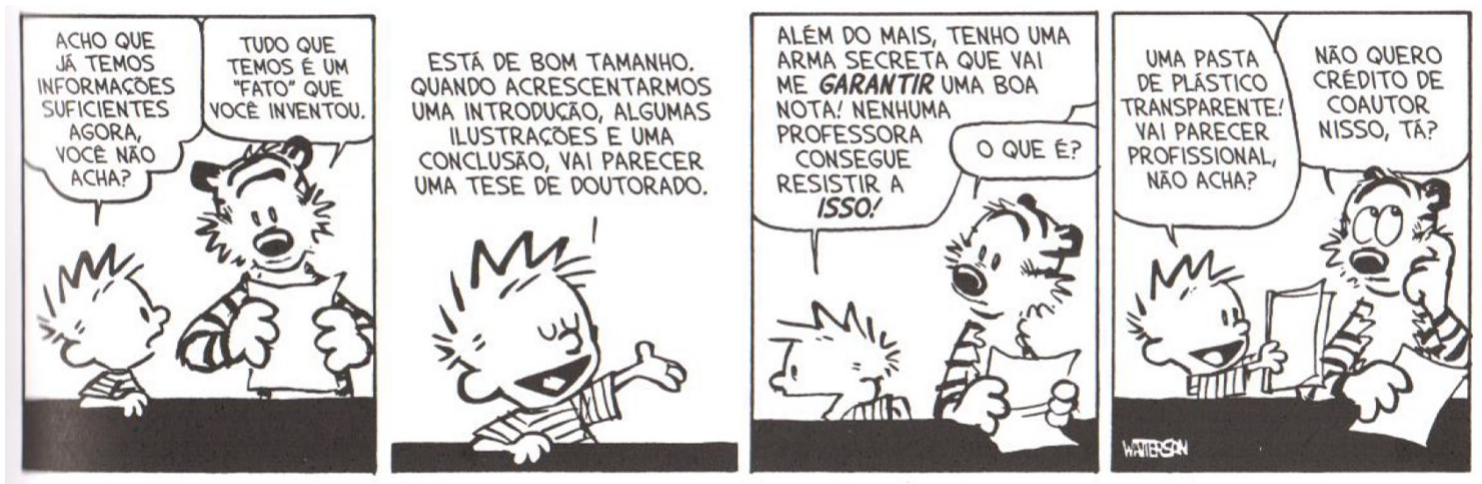


\section{RESUMO}

Problemas de otimização se fazem cada vez mais presentes nos mais diversos ramos da Engenharia. Encontrar configurações ótimas para um determinado problema significa, por exemplo, melhorar desempenho, reduzir custos entre outros ganhos. Existem hoje diversas maneiras de atacar um problema de otimização, cada qual com suas particularidades, vantagens e desvantagens. Dentre os métodos de otimização que utilizam gradientes de sensibilidade, o cálculo numérico dos mesmos consiste em uma importante etapa do projeto que, dependendo do problema, pode acarretar em custos computacionais muito elevados inviabilizando a abordagem escolhida. Este trabalho visa desenvolver e apresentar uma nova metodologia para o cálculo desses gradientes de sensibilidade, com base no Método Adjunto. O Método Adjunto é um método amplamente estudado e com diversas aplicações principalmente em Engenharia Aeronáutica. Nesse trabalho, todo o conhecimento prévio é utilizado para a derivação do método para aplicá-lo a escoamentos viscosos e incompressíveis. É desenvolvido também o cálculo do gradiente de sensibilidade com respeito a parâmetros geométricos e não geométricos. Para validar a metodologia proposta são feitas simulações numéricas das equações governantes do escoamento e adjuntas utilizando dois códigos computacionais distintos, SEMTEX e FreeFem ++ , o primeiro baseado no Método dos Elementos Espectrais e o segundo no Método dos Elementos Finitos, mostrando assim a independência do Método Adjunto na sua formulação contínua em relação a métodos computacionais. Para a validação são escolhidos problemas simples cujos gradientes possam ser calculados de outras formas permitindo comparações para calibrar e aperfeiçoar o cálculo do gradiente de sensibilidade

Palavras chave: otimização, Método Adjunto, CFD, escoamento incompressível. 


\section{ABSTRACT}

Optimization problems are widely present in differents fields of Engineering. Finding optimal configurations in a problem means, for example, improving performance, reducing costs, among other achievements. There are several wellknown ways to tackle an optimization problem, each one has its own advantages and disadvantages. Considering the gradient-based optimization methods, the step of their numerical calculation is extremely important, as it may result in huge computational costs, thus making the chosen method impracticable. This work aims to develop and present a new methodology to compute these sensitivity gradients based on the Adjoint Method. The Adjoint Method is a widely studied method with several applications chiefly in Aeronautical Engineering. In the present work, all the previous knowledge will be used to derive the equations of the method in order to apply them to viscous incompressible flows. The calculation of the sensitivity gradient, with respect to both geometric and non-geometric paramatersm will be developed as well. To validate the proposed methodology, numerical simulations of the governing and adjoint equations are carried out, using two computational codes called SEMTEX and FreeFem ++ , the former is based on the Spectral Element Method and the later, on the Finite Element Method, thus showing that the Adjoint Method, in its continuous formulation, is independent of the particular numerical method that is used. In order to validate the algorithm, simple problems are chosen, for which the gradients can be computed by other methods. This choice admits comparison between numerical values of gradients in order to calibrate and improve the methodology proposed.

Key words: optimization, Adjoint Method, CFD, incompressible flow. 


\section{SUMÁRIO}

Lista de Figuras vii

$\begin{array}{ll}\text { Lista de Tabelas ix } & \text { ix }\end{array}$

Lista de Símbolos $\quad$ x

1 Introdução 1

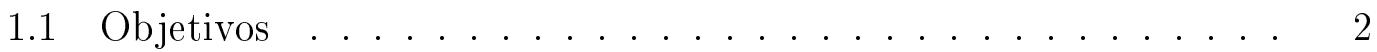

1.2 Escopo . . . . . . . . . . . . . . . . . . . 3

1.3 O Método Adjunto . . . . . . . . . . . . . . . . 5

1.4 Ciclo de Projeto . . . . . . . . . . . . . . . . . . . . 7

$\begin{array}{llr}2 & \text { Revisão Bibliográfica } & 9\end{array}$

2.1 Otimização de Sistemas Fluido-Mecânicos . . . . . . . . . . . 9

2.2 Método Adjunto . . . . . . . . . . . . . . . . . . . . . . 11

2.3 Conclusões . . . . . . . . . . . . . . . . . . . . . . . . . . . . 14

3 O Método Adjunto $\quad 16$

3.1 Formulação Geral . . . . . . . . . . . . . . . . . . . . . . . 16

3.2 Navier-Stokes Incompressível . . . . . . . . . . . . . . . . . . . . 21

3.2.1 Considerações Preliminares . . . . . . . . . . . . . . . 21

3.2.2 Equações Adjuntas . . . . . . . . . . . . . . . . . . . . . . 24

3.2.3 Condições de Contorno . . . . . . . . . . . . . . . . . . 31

3.3 Sensibilidades Não-Geométricas . . . . . . . . . . . . . . . . 38

3.3.1 Metodologia de Cacuci . . . . . . . . . . . . . . 40

3.3.2 Reformulação das Condições de Contorno . . . . . . . . . 41 
3.3.3 Considerações Sobre a Derivação . . . . . . . . . . . . 46

4 Simulações Numéricas $\quad 50$

4.1 Modelo Matemático do Escoamento . . . . . . . . . . . . . . 51

4.2 Equações Adimensionais . . . . . . . . . . . . . . . . . . . 51

4.3 Método dos Elementos Finitos . . . . . . . . . . . . . . . . 52

4.3.1 Método dos Resíduos Ponderados . . . . . . . . . . . 53

4.3.2 Formulação de Galerkin . . . . . . . . . . . . . . . . . 54

4.3.3 Método dos Elementos Espectrais para Navier-Stokes . . . 55

$\begin{array}{llr}5 & \text { Análise de Sensibilidade } & 60\end{array}$

5.1 Validação da Solução Adjunta . . . . . . . . . . . . . . . . . . 60

5.2 Sensibilidades não geométricas . . . . . . . . . . . . . . . 62

5.2.1 Escoamento Através de Um Canal 2-D . . . . . . . . . . 62

5.2.2 Escoamento Através de Um Canal 2-D - Variação de Espessura ...................... 75

5.3 Sensibilidades geométricas . . . . . . . . . . . . . . 81

5.3.1 Escoamento Através de um Canal com Restrição . . . . . . 81

6 Conclusões e Trabalhos Futuros $\quad 90$

6.1 Conclusões . . . . . . . . . . . . . . . . . . . . . . 90

6.2 Trabalhos Futuros . . . . . . . . . . . . . . . . . . . 92

$\begin{array}{ll}\text { Referências } & 93\end{array}$

Apêndice A - Equações de Navier-Stokes Generalizadas $\quad 98$

A.1 Introdução . . . . . . . . . . . . . . . . . . . . . . . 98

A.2 Álgebra Tensorial Generalizada . . . . . . . . . . . . . . . 100

A.3 Navier-Stokes Reduzido. . . . . . . . . . . . . . . . 106

A.4 Conclusões . . . . . . . . . . . . . . . . . . . . . . . . . . . . 108 


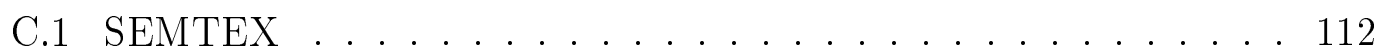

C.1.1 Quantidade de Iterações . . . . . . . . . . . . . . . . . 112

C.1.2 Grau do Polinômio . . . . . . . . . . . . . . . . . . 113

C.1.3 Passo de Integração . . . . . . . . . . . . . . . . . . . . . 114

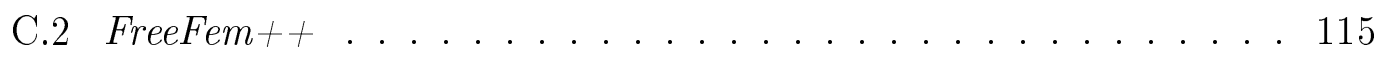

C.2.1 Quantidade de Elementos . . . . . . . . . . . 115

$\begin{array}{ll}\text { Apêndice D - Redução do Gradiente } & 118\end{array}$

D.1 Introdução . . . . . . . . . . . . . . . . . . . . . . . . 118

D.2 Variação do Jacobiano . . . . . . . . . . . . . . . . . . . . . . 122

D.3 Variação de $J \beta_{j^{\prime}}^{i} \ldots \ldots \ldots$. . . . . . . . . . . . . . . 123

D.4 Pseudo-Variações Físicas . . . . . . . . . . . . . . . . . . . . . . . 124

D.5 Integração por Partes . . . . . . . . . . . . . . . . . . . . . . 129

D.6 Conclusões . . . . . . . . . . . . . . . . . . . . . . . 130 


\section{LISTA DE FiguRAS}

1.1 Ciclo de Projeto . . . . . . . . . . . . . . . . 8

5.1 Solução Escoamento e Adjunta - P.50 . . . . . . . . . . . . . . 61

5.2 Escoamento Através de um canal 2D . . . . . . . . . . 63

5.3 Malha Computacional - Canal 2D ............ 66

5.4 Solução Escoamento e Adjunta - P.10 . . . . . . . . . . . . . . . 67

5.5 Gradientes - Canal 2D - Semtex - Adjunto x DF . . . . . . . . 68

5.6 Gradientes - Canal 2D - Semtex - Adjunto x Analitico . . . . . . 68

5.7 Gradientes - Canal 2D - Semtex - Adjunto x DF - $U_{\infty}=2,0 \mathrm{~m} / \mathrm{s} . \quad 69$

5.8 Gradientes - Canal 2D - Semtex - Adjunto x Analitico - $U_{\infty}=2,0 \mathrm{~m} / \mathrm{s} 70$

5.9 Malha Computacional - Canal 2D - FreeFem $++\ldots . . . . . .70$

5.10 Solução Escoamento e Adjunta - P.10 . . . . . . . . . . . . . 71

5.11 Gradientes - Canal 2D - FreeFem ++ - Adjunto x DF . . . . . . 72

5.12 Gradientes - Canal 2D - FreeFem ++ - Analitico x DF . . . . . . 72

5.13 Gradientes - Canal 2D - FreeFem ++ - Adjunto x DF $-U_{\infty}=2,0 \mathrm{~m} / \mathrm{s} 73$

5.14 Gradientes - Canal 2D - FreeFem ++ - Analitico x DF $-U_{\infty}=2,0 \mathrm{~m} / \mathrm{s} 73$

5.15 Gradientes - Canal 2D - FreeFem $++\mathrm{x}$ Semtex . . . . . . . . . 74

5.16 Gradientes - Canal 2D - FreeFem $++\mathrm{x}$ Semtex $-U_{\infty}=2,0 \mathrm{~m} / \mathrm{s} . .75$

5.17 Escoamento Através de um canal 2D com variação de espessura . 75

5.18 Malha Computacional - Canal 2D com variação de espessura . . . 76

5.19 Solução Escoamento e Adjunta - P.10 . . . . . . . . . . . . . 77

5.20 Gradientes - Canal 2D com Variação de Espessura - SEMTEX Adjunto x DF . . . . . . . . . . . . . . . 78

5.21 Malha Computacional - Canal 2D com Variação de Espessura FreeFem+t . . . . . . . . . . . . . . . . . 78 
5.22 Solução Escoamento e Adjunta - P.10 . . . . . . . . . . . . . . 79

5.23 Gradientes - Canal 2D com Variação de Espessura - FreeFem ++ Adjunto x DF . . . . . . . . . . . . . . . . . . . 80

5.24 Gradientes - Canal 2D com Variação de Espessura - FreeFem ++ x Semtex . . . . . . . . . . . . . . . . 80

5.25 Escoamento Através de um canal 2D com variação de espessura . 81

5.26 Malha Computacional - Canal 2D com Restrição . . . . . . . . . . 84

5.27 Solução Escoamento e Adjunta - P.10 . . . . . . . . . . . . . . . . 85

5.28 Gradientes - Canal 2D com Restrição - Parâmetro $a_{k_{0}}$ - Adjunto x

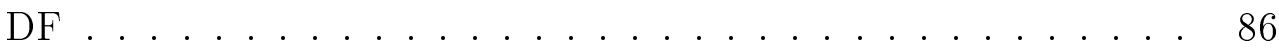

5.29 Gradientes - Canal 2D com Restrição - Parâmetro $a_{k_{1}}$ - Adjunto x

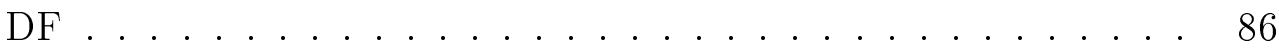

5.30 Gradientes - Canal 2D com Restrição - Parâmetro $a_{k_{0}}$ - Adjunto Reduzido x DF .................... . . 87

5.31 Gradientes - Canal 2D com Restrição - Parâmetro $a_{k_{1}}$ - Adjunto Reduzido x DF ...................... . 88

5.32 Gradientes - Canal 2D com Restrição - Parâmetro $a_{k_{0}}$ - Estendido x Reduzido . . . . . . . . . . . . . . . . . . 88

5.33 Gradientes - Canal 2D com Restrição - Parâmetro $a_{k_{1}}$ - Estendido x Reduzido . . . . . . . . . . . . . . . . . . . . . 89

A.1 Espaços físico e transformado . . . . . . . . . . . . . . . . . . . . 99 


\section{LISTA DE TABELAS}

3.1 Condições de Contorno . . . . . . . . . . . . . . . . . . . . . . 47

4.1 Coeficientes do esquema stiffly stable . . . . . . . . . . . . . . 58

C.1 Teste de Convergência - N - Canal 2D . . . . . . . . . . . . . . 113

C.2 Teste de Convergência - N - Canal 2D com Variação de Espessura 113

C.3 Teste de Convergência - P - Canal 2D . . . . . . . . . . . . . . 114

C.4 Teste de Convergência - P - Canal 2D com Variação de Espessura 114

C.5 Teste de Convergência - $\Delta t$ - Canal 2D . . . . . . . . . 115

C.6 Teste de Convergência - $\Delta t$ - Canal 2D com Variação de Espessura 115

C.7 Teste de Convergência - $N_{\text {entrada }}$ - Canal 2D . . . . . . . . . . 116

C.8 Teste de Convergência - $N_{\text {saida }}$ - Canal 2D com Variação de Espessura116

C.9 Teste de Convergência - $N_{\text {topo/baixo }}$ - Canal 2D com Variação de Espessura . . . . . . . . . . . . . . . . 116

C.10 Teste de Convergência - $N_{\text {entrada }}$ - Canal 2D com Variação de Espessura .......................... 116

C.11 Teste de Convergência - $N_{\text {saida }}-$ Canal 2D . . . . . . . . . . 117

C.12 Teste de Convergência - $N_{\text {topo/baixo }}-$ Canal 2D . . . . . . . . . 117 


\section{LisTa DE SÍMBolos}

X Vetor do espaço de estados

$\mathcal{A} \quad$ Variáveis do escoamento

$\mathcal{B} \quad$ Parâmetro qualquer do problema

$\mathcal{C}$ Geometria das fronteiras do escoamento

$\mathcal{J} \quad$ Funcional aumentado

$\mathcal{N} \quad$ Equações de Navier-Stokes

$\mathcal{X} \quad$ Vetor de coordenadas do problema

$\nu \quad$ Viscosidade cinemática

$\Omega \quad$ Domínio do escoamento

$\vec{e} \quad$ Determinada direção do escoamento

$\vec{n} \quad$ Direção normal a um corpo ou ao escoamento

$G \quad$ Força exercida por um fluido em um corpo em uma dada direção $\vec{e}$

I Medida de mérito

$a^{k} \quad$ Parâmetros de controle

$\mathrm{d} A \quad$ Elemento de Área

$e^{i j k} \quad$ Símbolo de Permutação

$g^{i j} \quad$ Componente contravariante do tensor métrico

$g^{i j} \quad$ Tensor métrico

$J \quad$ Jacobiano da transformação

L,S Operadores associados às equações de Navier-Stokes

$p \quad$ Pressão 
Q Termo forçante

T Período

$t \quad$ tempo

u Vetor velocidade

$v \quad$ Volume específico

$x^{j^{\prime}} \quad$ Coordenadas cartesianas

\section{Símbolos Gregos}

$\beta_{i}^{j^{\prime}} \quad$ Operador de transformação

$\delta_{j}^{i} \quad$ Delta de Kronecker

$\epsilon^{i j k} \quad$ Tensor de permutação em componentes contravariantes

$\Gamma_{i j k} \quad$ Símbolo de Christoffel

$\mu \quad$ Viscosidade

$\boldsymbol{\psi} \quad$ Vetor de velocidades adjuntas

$\boldsymbol{\sigma} \quad$ Tensor das tensões

$\boldsymbol{\tau}$ Tensor das tensões viscosas

$\theta \quad$ Pressão adjunta

$\xi^{i} \quad$ Coordenadas generalizadas 


\section{INTRODUÇÃO}

A engenharia como ciência desenvolve modelos matemáticos para descrever situações e problemas reais. Dessa forma é possível prever, projetar e estudar diferentes situações. Quanto mais o modelo conseguir reproduzir a realidade, melhor ele se torna para o propósito para o qual ele foi criado. Com a evolução tecnólogica que trouxe novas ferramentas para o estudo, projeto e previsão de fenômenos, as pesquisas em engenharia ganharam importantes aliadas para validar modelos já existentes, aperfeiçoá-los, criar novos ou ainda explorar novos problemas.

Porém o desafio não se limita somente a desenvolver modelos capazes de estudar as mais diferentes situações. Outro ponto de interesse é desenvolver modelos que encontrem uma configuração ótima do problema. Em outras palavras, problemas de otimização. A otimização não se limita somente à engenharia, mas dentro da mesma, nas mais diferentes áreas, encontram-se problemas com esse objetivo, nos quais se deseja encontrar qual configuração de parâmetros relacionados ao problema irão levar a um valor máximo ou mínimo de uma determinada função previamente especificada. Maximizar o rendimento mecânico de um equipamento, minimizar o gasto energético de uma instalação e minimizar forças viscosas em corpos que se movimentam em fluidos são exemplos de problemas de otimização.

Dentro do contexto da Mecânica de Fluidos, no qual este trabalho se encaixa, há uma extensa gama de problemas de otimização. Entretanto, devido à complexidade matemática da maioria dos modelos utilizados para descrever escoamentos, não é fácil estudar tais problemas do ponto de vista analítico. Durante muitos anos, a alternativa tem sido o estudo experimental, que permite o estudo de escoamentos de forma muito mais próxima da realidade. Alternativa essa extremamente importante até os dias de hoje em diferentes situações e utilizada também na obtenção de resultados para fins comparativos com outros tipos de modelos.

Em algumas situações, o estudo experimental é caro e exige uma grande es- 
trutura para ser realizado. Nesse cenário, o crescimento do uso do computador se tornou mais um importante aliado nesses estudos pois possibilitou a implementação de métodos e modelos numéricos para o estudos de escoamentos. Nasceu assim a Dinâmica dos Fluidos Computacional, mais conhecida pela sua sigla CFD ${ }^{1}$.

A CFD desempenha papel cada vez mais relevante na engenharia. Sua função é especialmente importante na fase de concepção de sistemas e equipamentos, por permitir que se analisem configurações alternativas a custos relativamente baixos. A combinação de ferramentas de simulação do escoamento com métodos de projeto inverso e otimização pode ter grande impacto nesta etapa do ciclo de projeto.

As últimas três décadas testemunharam intensa atividade de pesquisa no sentido de responder a essa demanda, com resultados fartamente documentados na literatura. Dentre as abordagens mais relevantes do problema, aquela que ficou conhecida como Método Adjunto se destaca por permitir excepcional redução do custo computacional no cálculo do gradiente de sensibilidade. Também são seus atrativos o fato de que não se prende a um modelo particular da física do escoamento, e de que pode servir tanto à finalidade de projeto inverso como a de otimização. Tais características fizeram do método adjunto um prolífico tema de pesquisa.

Nos últimos anos, o grupo de pesquisa do qual faço parte tem desenvolvido aplicações do método adjunto para escoamentos compressíveis não viscosos, nos regimes sub e supersônico. Este trabalho representa uma continuidade das atividades de pesquisa ao aplicar o método adjunto para escoamentos viscosos incompressíveis. Os objetivos do trabalho estão descritos a seguir.

\section{$1.1 \quad$ Objetivos}

Este trabalho tem os seguintes objetivos:

- Obter as equações adjuntas para escoamentos incompressíveis bem como o conjunto de condições de contorno correspondente.

- Desenvolver metodologia para cálculo de sensibilidades de funções objetivo em relação a parâmetros geométricos e não geométricos para escoamentos viscosos incompressíveis. Esta última é a principal contribuição do trabalho.

\footnotetext{
${ }^{1}$ (do inglês Computational Fluid Dynamics)
} 
- Obter a formulação reduzida para sensibilidades em relação a parâmetros geométricos (Apêndice D)

- Validar as metodologias propostas comparando os gradientes calculados com outros métodos de cálculo (diferenças finitas e aproximações teóricas).

Nos últimos anos, nosso grupo tem desenvolvido aplicações do método adjunto para escoamentos compressíveis não viscosos, nos regimes sub e supersônico. Na continuidade das atividades de pesquisa, este trabalho tem por finalidade o desenvolvimento de uma metodologia de cálculo de sensibilidades geométricas e não geométricas para escoamentos viscosos incompressíveis de modo que possam ser, futuramente, utilizadas em problemas de otimização. Vale ressaltar que o cálculo de sensibilidades é uma etapa muito importante em um problema de otimização. Desse modo será possível, também, implementar a abordagem propostas em diversos problemas de relevância para diferentes áreas da engenharia tais como aeronáutica, petróleo, biomédica entre outras.

\subsection{Escopo}

O objetivo deste trabalho é desenvolver uma metodologia de cálculo de sensibilidades geométricas e não geométricas aplicável a escoamentos viscosos incompressíveis. Como já mencionado, tal metodologia será desenvolvida via Método Adjunto, utilizando sua formulação contínua.

O estudo, que é aqui apresentado, discute toda a derivação do método voltada para a aplicação em escoamentos viscosos incompressíveis. Dessa forma, englobará a definição da medida de mérito, obtenção das equações adjuntas, estabelecimento das condições de contorno e a equação para o cálculo do gradiente de sensibilidade da medida de mérito em relação a quaisquer parâmetros do escoamento.

Serão utilizados dois códigos computacionais. O primeiro deles, o SEMTEX, foi desenvolvido por H. M. Blackburn (BARKLEY; BLACKBURN; SHERWIN, 2002) que desenvolve colaborações com o grupo de pesquisa do Prof. S. Sherwin do Imperial College. Este último professor, por sua vez, desenvolve intensa colaboração de pesquisas com os Profs. J. R. Meneghini e B. Carmo do PME-EPUSP. Assim, através deles entrou-se em contato com um membro do grupo do Prof. Sherwin, o Dr. Xuerui Mao (MAO, 2010), que permitiu fazer uso do referido código para desenvolver as aplicações que são o objetivo desse trabalho. O código resolve 
as equações de Navier-Stokes e adjuntas utilizando o Método de Elementos Espectrais (ou método $h p$, como é também conhecido). Esse método, de forma bastante simplificada, é a combinação de métodos espectrais na sua forma pura e do método de elementos finitos. Dessa combinação resulta o Método de Elementos Espectrais que conta com a convergência exponencial oriunda dos métodos espectrais e que trabalha com a divisão do domínio computacional em elementos, tal como no método de elementos finitos. O segundo código, o FreeFem++, é um código baseado no Método dos Elementos Finitos (HECHT, 2012) que resolve as equações de Navier-Stokes utilizando o Método dos Resíduos Ponderados. O estudo aprofundado e detalhado destes métodos não faz parte do escopo desse trabalho, assim sendo serão apresentados no capítulo 4 somente alguns conceitos fundamentais dos mesmos que são relevantes para o presente trabalho.

De posse das soluções do escoamento e adjunta é possível prosseguir nas etapas do loop de projeto, apresentado na seção 1.4. Calcula-se o gradiente de sensibilidade da medida de mérito escolhida em relação a um ou mais parâmetros do escoamento. Esse cálculo, seguindo a metodologia proposta nesse trabalho, apresenta um custo computacional muito inferior se comparado ao mesmo cálculo via diferenças finitas. O motivo dessa redução no custo computacional será explicado no final da seção 1.3. O gradiente de sensibilidade via diferenças finitas é calculado da seguinte forma:

$$
\frac{\partial I}{\partial \mathcal{B}_{i}}=\frac{I\left(\mathcal{B}_{i}+\delta \mathcal{B}_{i}\right)-I\left(\mathcal{B}_{i}\right)}{\delta \mathcal{B}_{i}}
$$

Onde $I$ é a medida de mérito escolhida, $\mathcal{B}_{\rangle}$é um parâmetro qualquer do problema e $\delta \mathcal{B}\rangle$ representa uma variação infinitesimal desse parâmetro e $i$ é o índice do parâmetro escolhido. Para calcular o gradiente de sensibilidade para cada parâmetro em um problema que possui $n$ deles, serão necessárias $(2 n+1)$ simulações computacionais do escoamento para diferenças finitas centradas e $(n+$ 1) simulações no caso de diferenças finitas de 1 ordem. Utilizando a metodologia que será, aqui, proposta, esse cálculo terá o custo de apenas 2 (duas) simulações: solução base do escoamento e equações adjuntas. Isso é verdade uma vez que o gradiente em relação a qualquer parâmetro, seja qual for sua equação, depende exclusivamente de variáveis das soluções do escoamento e adjunta.

Inicialmente serão estudados casos simples de escoamentos viscosos incompressíveis com o intuito de validar a metodologia proposta. Para tal faz-se a comparação dos resultados obtidos com soluções analíticas (quando houver) e/ou 
via diferenças finitas. Finalizada a validação pode-se partir para o estudo de problemas mais desafiadores, ou seja, problemas com funções objetivo que dependam de muitos parâmetros.

\section{$1.3 \quad$ O Método Adjunto}

Aqui é apresentada uma formulação geral do método adjunto. A formulação específica voltada para escoamentos viscosos incompressíveis está descrita no capítulo (3)

De modo geral, medidas de mérito relevantes às aplicações fluido-dinâmicas envolvem funcionais ou funções que dependem das variáveis do escoamento $\mathcal{A}$ neste caso vetor de velocidades e pressão - e da geometria de suas fronteiras $\mathcal{C}$ (JAMESON; KIM, 2003b; REUTHER, 1996).

$$
I=I(\mathcal{A}, \mathcal{C})
$$

Em geral, $\mathcal{C}$ pode ser representada como função das coordenadas $\mathcal{X}$, especificada por um conjunto de parâmetros $\mathcal{B}: \mathcal{C}=\mathcal{C}(\mathcal{X}, \mathcal{B})$. Variações da geometria $\delta \mathcal{C}$ podem ser produzidas por alterações nos parâmetros $\delta \mathcal{B}$ e, naturalmente, elas implicam em variações no campo do escoamento $\delta \mathcal{A}$. Assim, pode-se escrever

$$
\delta I=\frac{\partial I}{\partial \mathcal{A}}^{T} \delta \mathcal{A}+\frac{\partial I}{\partial \mathcal{C}}^{T} \delta \mathcal{C}
$$

Em princípio, a sensibilidade da medida de mérito a variações da geometria é medida pelo gradiente

$$
\frac{\partial I}{\partial \mathcal{B}}=\frac{\partial I^{T}}{\partial \mathcal{A}} \frac{\partial \mathcal{A}}{\partial \mathcal{B}}+\frac{\partial I^{T}}{\partial \mathcal{C}} \frac{\partial \mathcal{C}}{\partial \mathcal{B}}
$$

Para $\mathcal{C}(\mathcal{X}, \mathcal{B})$ conhecida, as derivadas $\partial \mathcal{C} / \partial \mathcal{B}$ podem ser estimadas sem maiores dificuldades. Entretanto o termo $\partial \mathcal{A} / \partial \mathcal{B}$ tem avaliação mais complicada, uma vez que, em geral, não se conhece explicitamente a dependência das variáveis do escoamento com respeito aos parâmetros que controlam a geometria. Há ainda a questão da realizabilidade das variações no campo do escoamento $\delta \mathcal{A}$, que não foi imposta à eq. (1.4). Essa realizabilidade consiste em garantir que os campos de velocidade e pressão obtidos com as suas respectivas variações atendam às equações que governam o escoamento.

O método de diferenças finitas apresentado anteriormente na eq. (1.2) procedimento assegura a realizabilidade das variações $\delta \mathcal{A}$, à medida em que busca 
uma solução convergida do escoamento para cada uma das perturbações. Entretanto, ele o faz a custa de grande aumento do esforço computacional, justamente porque requer pelo menos uma solução convergida para cada perturbação. É evidente que o custo computacional desta abordagem fica proibitivo à medida em que aumenta o número de parâmetros que controlam $\mathcal{C}(\mathcal{X}, \mathcal{B})$.

Em princípio, uma forma de contornar essa dificuldade seria restringir a priori as variações das grandezas físicas $\delta \mathcal{A}$ ao espaço das soluções realizáveis. $\mathrm{O}$ método adjunto faz justamente isso, ao impor as equações que governam o escoamento como restrições ao problema variacional. Para tanto, considera-se que a solução dessas equações depende das variáveis do escoamento e da forma de suas fronteiras, em termos gerais,

$$
\mathbf{N}(\mathcal{A}, \mathcal{C})=0
$$

Portanto, todas as variações realizáveis $\delta \mathcal{A}$ devem satisfazer a condição $\delta \mathbf{N}=0$, isto é

$$
\delta \mathbf{N}=\frac{\partial \mathbf{N}}{\partial \mathcal{A}} \delta \mathcal{A}+\frac{\partial \mathbf{N}}{\partial \mathcal{C}} \delta \mathcal{C}=0
$$

A restrição é introduzida no problema fazendo-se o produto da eq. (1.6) por um multiplicador de Lagrange $\Psi$ e somando-a a eq. (1.3). Com isso se obtém uma variação funcional aumentada que, a princípio, restringe $\delta \mathcal{A}$ ao espaço das soluções realizáveis $-\delta \mathcal{A} \Rightarrow \delta \mathbf{N}=0$.

$$
\begin{aligned}
& \delta I=\frac{\partial I}{\partial \mathcal{A}}^{T} \delta \mathcal{A}+\frac{\partial I}{\partial \mathcal{C}}^{T} \delta \mathcal{C}-\Psi^{T}\left(\frac{\partial \mathbf{N}}{\partial \mathcal{A}} \delta \mathcal{A}+\frac{\partial \mathbf{N}}{\partial \mathcal{C}} \delta \mathcal{C}\right) \\
& =\underbrace{\left[\frac{\partial I^{T}}{\partial \mathcal{A}}-\Psi^{T} \frac{\partial \mathbf{N}}{\partial \mathcal{A}}\right]}_{(a)} \delta \mathcal{A}+\underbrace{\left[{\frac{\partial I^{T}}{\partial \mathcal{F}}}^{T} \Psi^{T} \frac{\partial \mathbf{N}}{\partial \mathcal{C}}\right]}_{(b)} \delta \mathcal{C}
\end{aligned}
$$

No lado direito da segunda igualdade (1.7) agrupam-se os termos que envolvem variações da solução do escoamento $(a)$ e aqueles que envolvem variações de geometria (b). Escolhendo-se $\Psi$ de modo a satisfazer a equação

$$
\frac{\partial \mathbf{N}}{\partial \mathcal{A}} \Psi=\frac{\partial I}{\partial \mathcal{A}}
$$

elimina-se o termo (a) na eq.(1.7), e a variação da medida de mérito resulta numa expressão simplificada, que é independente de $\delta \mathcal{A}$

$$
\delta I=\left[{\frac{\partial I^{T}}{\partial \mathcal{F}}}^{T}-\Psi^{T} \frac{\partial \mathbf{N}}{\partial \mathcal{C}}\right] \delta \mathcal{C}
$$

A expressão (1.8) representa a equação adjunta. A simplificação que ela permite em (1.9) é consequência direta da imposição da realizabilidade de $\delta \mathcal{A}$, via eq. 
(1.6). Ao lado disso, a independência da variação $\delta I$ com relação a $\delta \mathcal{A}$ permite que se calcule o gradiente de sensibilidade com respeito a qualquer número de parâmetros $\mathcal{B}_{i}$, sem a necessidade de simulações adicionais do escoamento. Essa é a essência do método adjunto, proposto por Pirroneau (PIRRONEAU, 1973) inicialmente para problemas elípticos e posteriormente para problemas hiperbólicos por Jameson (JAMESON, 1988; JAMESON, 1994).

Ainda numa forma genérica, a eq. (1.8) permite antever algumas propriedades fundamentais da equação adjunta: ambos os termos $(\partial \mathbf{N} / \partial \mathcal{A})$ e $(\partial I / \partial \mathcal{A})$ são independentes de $\Psi$, de onde resulta sua linearidade; sua estrutura concentra no lado esquerdo os termos que dependem apenas das equações que governam o escoamento. O termo não homogêneo, no lado direito, é o único que envolve a particular medida de mérito adotada.

Se, por um lado, a linearidade traz benefícios claros para as aplicações; por outro, a estrutura da equação adjunta tem profundas consequências na formulação do método. A separação entre os termos que dependem das equações da mecânica dos fluidos e os que envolvem a medida de mérito implica na existência de um operador adjunto associado a cada modelo da física do escoamento. Ao menos em princípio, então, em se obtendo o operador associado ao modelo em questão, poder-se-ia otimizar uma ampla gama de medidas de mérito, pois isto envolveria modificar apenas o termo não homogêneo. Outra consequência imediata desta separação é que, em grande medida, o algoritmo para resolver a eq. (1.8) depende do particular operador adjunto considerado, e não do termo forçante. Isso permite enorme flexibilidade na definição das medidas de mérito.

\subsection{Ciclo de Projeto}

A metodologia de otimização segue o seguinte ciclo, ou loop exibido na figura 1.1:

O ciclo de projeto inicia com a escolha de um problema e sua respectiva geometria. No passo seguinte gera-se a malha computacional. Nesse trabalho as malhas computacionais foram geradas no programa comercial Gambit. Seguindo o ciclo, a próxima etapa é obter a solução do escoamento. Nesse trabalho, como já mencionado, são utilizados os códigos SEMTEX e FreeFem ++ nessa etapa. Logo após a solução do escoamento, são resolvidas as equações adjuntas, utilizando novamente ambos os códigos citados. Em seguida, avalia-se o gradiente da medida de mérito em relação a um determinado parâmetro do projeto. Caso o critério de 


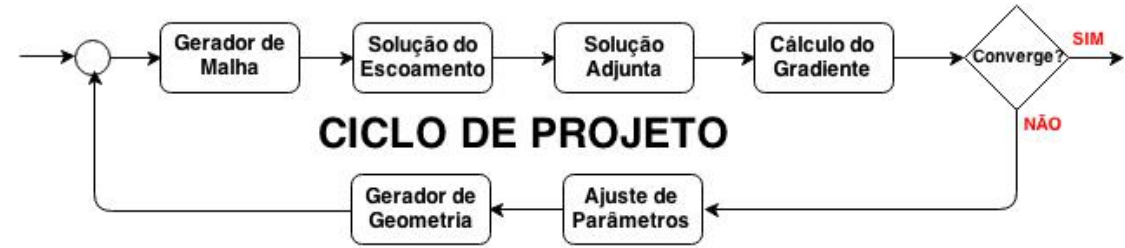

Figura 1.1: Loop de testes 2-D para rotina de otimização e projeto inverso baseada no método adjunto. Os ciclos de otimização envolvem uma seqüência de: geração da malha, simulação do escoamento, solução adjunta, avaliação do gradiente e eventual modificação de geometria para satisfazer a medida de mérito.

convergência seja atendido, o loop é finalizado. Caso não seja atendido, efetuamse mudanças nos parâmetros do problema para chegar a uma nova configuração de geometria e reinicia-se o ciclo até a convergência do módulo do gradiente. É bom frisar que realizar o loop completo de otimização não está no escopo deste trabalho. Aqui apresenta-se o ciclo completo para ilustrar onde a etapa de cálculo de sensibilidade (essa sim faz parte do escopo da tese) se encaixa em um problema de otimização. 


\section{REVISÃo BIBLIOGRÁFICA}

\subsection{Otimização de Sistemas Fluido-Mecânicos}

O enorme progresso experimentado pela CFD ensejou uma revolução na metodologia de projeto de sistemas fluido-dinâmicos. O desenvolvimento de métodos robustos e confiáveis e a crescente disponibilidade de recursos computacionais deram-lhe o status de ferramenta de uso corrente na indústria, desempenhando função complementar à atividade experimental (MACCORMACK, 1993).

É especialmente na fase de concepção do projeto, que as simulações computacionais têm maior impacto, à medida em que permitem analisar configurações alternativas a custos significativamente mais baixos que a experimentação(GILES, 1997; JAMESON, 1997). Ainda assim, a estratégia geralmente adotada nessa etapa continua a ser a tentativa e erro. Isto é, propõe-se uma configuração e analisamse os resultados das simulações, com vistas a obter o efeito desejado (SANTOS, 1993). Por exemplo, considerando um problema onde deseja-se encontrar um perfil de asa que maximize a sustentação. Resolver esse problema com tentativa e erro consistiria em realizar várias simulações com diferentes configurações de perfil e para cada uma delas calcular a sustentação. Analisam-se os resultados e o perfil que apresentar a maior sustentação é considerado o perfil ótimo.

Essa abordagem depende em larga margem da experiência acumulada do projetista, e suas chances de sucesso diminuem sensivelmente à medida em que se impõe condições mais elaboradas ao projeto. Isso ocorre porque não há como garantir que um método de tentativa e erro explore adequadamente o espaço de soluções viáveis. Os métodos de projeto inverso e otimização podem ter um impacto muito positivo nessa situação. Combinados com recursos de CFD, eles permitem uma exploração mais eficiente e sistemática do espaço de soluções de projeto.

Uma caracterização geral dos métodos de projeto inverso e otimização fluidodinâmicos pode ser encontrada em (SANTOS, 1995). Os primeiros distinguem-se por comparar a distribuição de uma quantidade física de interesse a uma distri- 
buição desejada, especificada previamente pelo usuário - por exemplo a pressão. Com base nessa comparação, os métodos de projeto inverso estimam alterações da geometria que produziriam o efeito desejado. Em geral, não há garantia de que a distribuição desejada seja factível, ou que implique em melhor desempenho, uma vez realizada. Além disso, não se pode garantir que a configuração obtida represente uma solução ótima para as condições prescritas. Assim, a experiência acumulada mantém sua relevância, apenas num nível diferente. Não mais se procura especificar uma geometria que permita alcançar efeitos desejáveis, mas uma distribuição objetivo que corresponda a tais efeitos.

Os métodos de otimização, por outro lado, proporcionam uma abordagem mais abrangente do projeto fluido-dinâmico. Em essência, esses métodos buscam extremos de funções de mérito previamente definidas. Os extremos representam pontos de máximo ou mínimo locais, no espaço de projeto. As funções de mérito permitem definições bastante variadas: podem representar forças fluidodinâmicas, ou mesmo uma medida do erro com respeito a uma distribuição desejada de pressões. Naturalmente, o último caso representa uma aplicação de projeto inverso que, colocada nesses termos, pode ser vista como um caso particular de otimização.

De qualquer modo, a existência de um extremo, ainda que local, deve corresponder a uma configuração ótima com respeito à particular medida de mérito adotada. A questão da realizabilidade é um pouco mais complexa. Em princípio, ela implica que se imponham restrições ao problema que a garantam. Em mecânica dos fluidos, as condições de realizabilidade são, em última análise, as equações que governam o escoamento. E a imposição destas como restrições ao problema de otimização depende da forma como o problema é equacionado.

A teoria de controle de sistemas governados por equações diferenciais (LIONS, 1971) proporciona os fundamentos conceituais e o formalismo necessários a essa atividade. O método proposto por Jameson (JAMESON, 1988) faz uso desses recursos com dupla vantagem: de um lado, ao impor as equações da mecânica dos fluidos como restrições ao problema variacional, projetam-se as variações da medida de mérito no espaço das soluções realizáveis; de outro, essas restrições permitem uma simplificação excepcional no cálculo do gradiente de sensibilidade, com a consequente redução do seu custo computacional. No que segue, apresentam-se os fundamentos conceituais do método proposto por Jameson, que ficou conhecido como método adjunto. Uma breve revisão bibliográfica acerca desse método é exibida no próximo item. 


\subsection{Método Adjunto}

Dentre as diversas abordagens possíveis para um problema de otimização, como foi visto, há os métodos de projeto inverso. Nesse contexto a utilização do Método Adjunto mostra-se bastante propensa pela sua flexibilidade quanto ao modelo físico do escoamento bem como na escolha de medidas de mérito a serem otimizadas.

O Método Adjunto foi proposto inicialmente por Pirroneau em uma série de artigos do autor. No primeiro, (PIRRONEAU, 1973) o autor deseja obter a geometria do corpo que apresenta mínimo arrasto em um escoamento com baixo número de Reynolds (Escoamento de Stokes). Para tal, utiliza métodos variacionais, que é o caso do Método Adjunto. No segundo artigo (PIRRONEAU, 1974) o autor estende a metodologia de solução do problema para escoamentos laminares com números de Reynolds superiores aos do seu primeiro trabalho nessa linha. Ambos os problemas abordados eram elípticos. O mesmo autor, dessa vez em um livro, conjuntamente com Mohamadi, (MOHAMADI; PIRRONEAU, 2001) apresenta métodos de otimização da geometria de corpos em relação a funções dadas. No capítulo 4 apresenta o Método Adjunto como método eficiente no cálculo de gradientes de sensibilidade face outros métodos conhecidos tais como diferenças finitas, passo complexo, etc. Dando continuidade às aplicações do Método Adjunto, Jameson utiliza o método para escoamentos transônicos regidos pelas equações de Euler (JAMESON, 1988). O objetivo do problema é propor modificações na geometria de aerofólios, por exemplo, para melhorar a performance da aeronave. Nesses primeiros trabalhos analisados destaca-se o potencial do Método Adjunto no cálculo de gradientes de sensibilidade, que é o objeto desse trabalho.

Com o passar dos anos, o Método Adjunto passou a ser objeto de diferentes linhas de pesquisa e empregado nas mais diferentes áreas e aplicações, indo desde reatores termo-nucleares a ciências atmosféricas. Dentre todas essas aplicações é interessante citar algumas. Em (ALONSO; KROO, 2002) o grupo desenvolve métodos de análise e projeto de aeronaves com foco na redução do estrondo sônico (ruído causado por ondas de choque). Dentre as frentes de trabalho dos autores há métodos de otimização baseados no cálculo de gradientes de sensibilidade. Nessas frentes o método adjunto foi empregado, aumentando assim a gama de parâmetros da aeronave que puderam ser modificados para melhorar a performance da mesma. No trabalho (HALL; CACUCI, 1983) os autores empregam o Método Adjunto também no cálculo de gradientes porém para modelos de fenômenos atmosféricos. 
Dentre as diversas aplicações do método, as relacionadas à aerodinâmica se destacam pelo seu desenvolvimento conjunto com métodos de otimização na área. O Método Adjunto mostra-se muito eficiente em projeto inverso e aplicações relativas à mudança de geometria dos problemas estudados. Em (DUTA; GILES; CAMPOBASSO, 2002) os autores utilizam o método adjunto para analisar e minimizar os efeitos da vibração nas pás de uma turbina. Em (KIM; NAKAHASHI, 2005) os autores desejam modificar a geometria de um aerofólio com flaps de modo a maximizar a sustentação sobre ele. No trabalho é desenvolvido um código baseado em um método de otimização por gradientes no qual o Método Adjunto, na sua forma discreta, é empregado por permitir maior flexibilidade quanto à complexidade da geometria a ser estudada sem elevar consideravelmente o custo computacional. Muito semelhante ao anterior, o artigo (KIM; ALONSO; JAMESON, 2004) também tem como objetivo a maximização da sustentação em um aerofólio com slat e flap. Para tal é derivado um método de otimização baseado no Método Adjunto para as equações de Navier-Stokes, porém utilizando a forma contínua do método. Para elevados números de Reynolds as equações constitutivas para o método passam a ser as equações médias de Reynolds ${ }^{1}$.

Há também aplicações do Método Adjunto em problemas com escoamentos transitórios, como em (NADARAJAH; JAMESON, 2007) onde é apresentado um método de controle ótimo para escoamentos transitórios, com base no Método Adjunto. Para validação do mesmo são apresentados os resultados da utilização do método em um aerofólio bidimensional oscilante. Em trabalho posterior (NADARAJAH; JAMESON, 2007) são apresentados o método e resultados para um aerofólio tridimensional. Outras aplicações do Método Adjunto consistem na análise e quantificação de erros nos funcionais (medidas de mérito) (GILES; PIERCE; SüLI, 2004),(GILES; SüLI, 2002) e em técnicas para redução dos mesmos tal como adaptação de malhas (VENDITTI; DARMOFAL, 1999), (VENDITTI; DARMOFAL, 2003) e (VENDITTI; DARMOFAL, 2000).

Dentro das aplicações aerodinâmicas presentes em literaturas aqui citadas, as medidas de mérito estudadas dependem das variáveis do escoamento e da localização das fronteiras (JAMESON; MARTINELLI; PIERCE, 1998; JAMESON; SRIRAM; MARTINELLI, 2003). Abaixo, alguns exemplos de medidas de mérito utilizadas

\footnotetext{
${ }^{1}$ Em inglês Reynolds-averaged Navier-Stokes - RANS
} 
em aplicações aerodinâmicas:

$$
I=\oint_{B} \frac{\left(C_{p}-C_{p t}\right)^{2}}{2} \mathrm{~d} S \quad ; \quad K=\int_{\mathcal{D}} \frac{\rho u^{2}}{2} \mathrm{~d} V \quad ; \quad \overline{C_{f}}=\frac{1}{T} \int_{0}^{T} \oint_{B} \frac{\mathbf{n} \cdot \boldsymbol{\sigma} \cdot \mathbf{e}}{q_{\infty}} \mathrm{d} S \mathrm{~d} t
$$

A primeira integral calcula o erro quadrático de uma distribuição de pressão $C_{p}$, em relação a uma distribuição previamente estabelecida como desejada $\left(C_{p t}\right)$, na superfície de um corpo $(B)$. Esse é um funcional de interesse em problemas de projeto inverso no qual deseja-se encontrar a geometria de uma asa que apresentará a distribuição de pressão desejada. A segunda integral representa a energia cinética de todo o domínio do escoamento $(\mathcal{D})$. Por fim, a terceira integral expressa a média temporal da força exercida pelo fluido na superfície do corpo $(\mathbf{n} \cdot \boldsymbol{\sigma})_{B}$, projetada numa dada direção $(\mathbf{e})$.

Em aplicações mais recentes, grupos de pesquisa estão utilizando o Método Adjunto no cálculo de gradientes para otimização geométrica de carros e outros veículos, com o objetivo de reduzir o arrasto aerodinâmico, o consumo de combustível e melhorar a eficiência desses veículos (XU; JAHN; MüLLER, 2013). Em (KARPOUZAS et al., 2015), o grupo também utiliza o método para a otimização geométrica de veículos e destaca a escassez de códigos comerciais que resolvam as equações adjuntas e computem sensibilidades geométricas. Neste artigo, o grupo desenvolve o código computacional em um software não comercial (código aberto), medida que também será adotada no presente trabalho. Alguns pesquisadores desse mesmo grupo exploraram outros problemas na área automotiva não se limitando apenas à redução de arrasto aerodinâmico em veículos automotores mas extendendo essas aplicações para outras medidas de mérito como, por exemplo, ruído sonoro causado pelo escoamento ao redor de espelhos retrovisores visando a redução desse ruído (KIACHAGIAS et al., 2015).

Na literatura é possível encontrar tanto em artigos mais antigos como em outros mais recentes, autores e grupos de pesquisa que ora utilizaram a formulação discreta do método adjunto ora utilizaram a formulação contínua. Há ainda, quem comparou ambas as formulações. Em (NADARAJAH; JAMESON, 2000) os autores comparam ambos os métodos em uma aplicação de redução de arrasto em perfis de aerofólios e concluem que a diferença entre os gradientes calculados entre os métodos contínuo e discreto é muito pequena em relação ao gradiente calculado via diferenças finitas e essa diferença se torna ainda menor a medida em que o tamanho da malha computacional aumenta. Em artigos mais recentes (PETER; 
DWIGHT, 2010), (KIACHAGIAS; GIANNAKOGLOU, 2015) é visto que aplicações e resultados com ambas as abordagens do Método Adjunto são válidas e apresentam resultados satisfatórios. Os gradientes calculados por ambos os métodos nas aplicações apresentadas nesses e em outros trabalhos apresentam valores bastante próximos (diferença inferior a 1\%) entre si e se comparados aos mesmos gradientes calculados via diferenças finitas ou outros métodos. De todos esses trabalhos é importante aproveitar algumas colocações e conclusões desses trabalhos que comparam ambas as abordagens. Para pontuar essas colocações é conveniente explicitar que a formulação contínua do método adjunto é obtida diretamente das equações do problema primal, ou seja as equações do escoamento, enquanto a formulação discreta é obtida da forma discretizada das equações do escoamento. Com isso, conclui-se que a implementação computacional da formulação contínua é mais fácil pois independe da discretização das equações do escoamento o que traz ainda uma flexibilidade e liberdade quanto ao uso do código computacional para resolver as equações adjuntas. Os artigos citados anteriormente atestam ainda que o custo computacional para resolver as equações adjuntas é menor no caso contínuo.

Concentrando os olhares para aplicações fluido-dinâmicas em especial para escoamentos incompressíveis regidos pelas equações de Navier-Stokes, destaca-se o trabalho de (KAVVADIAS; KIACHAGIAS; GIANNAKOGLOU, 2015) onde são apresentadas as expressões matemáticas para gradientes de sensibilidades em relação a parâmetros geométricos. É feita ainda uma comparação entre duas expressões para o mesmo gradiente: uma contendo integrais de superfície e volume e outra contendo somente integrais de superfície. O procedimento para obtenção do gradiente somente com integral de superfície é similar ao contido em (JAMESON; KIM, 2003a).

\subsection{Conclusões sobre a Revisão Bibliográfica}

Assim, a despeito dos enormes desafios indicados por este levantamento preliminar, verifica-se que o Método Adjunto mostra-se uma importante ferramenta nos problemas de otimização sobretudo naqueles baseados em gradientes. O método mais tradicional do cálculo dos mesmos consiste em utilizar diferenças finitas. Porém esse método requer, para cada parâmetro, uma simulação convergida do escoamento. Aumentando-se o número de parâmetros, o número de simulações necessárias e o custo computacional também aumenta.

Desse modo, para desenvolver uma metodologia de otimização para escoamen- 
tos viscosos incompressíveis, baseada no cálculo de gradientes, o método adjunto aparece como o mais indicado para esse propósito. Isso é verdade uma vez que permite uma exploração sistemática do espaço de soluções com grande fidelidade à física do escoamento, e com baixo custo computacional visto que para o cálculo dos gradientes de sensibilidade em relação a qualquer número de parâmetros serão necessárias apenas duas simulações computacionais o que representa um ganho significativo de custo. A metodologia seguirá com a idéia base exibida nos trabalhos citados nesse capítulo: impor as equações constitutivas como restrições do problema de otimização, eliminando, assim, a necessidade de mais simulações.

Este trabalho adotará a abordagem contínua do método pela facilidade na derivação das equações em comparação à abordagem discreta e também pela liberdade em relação ao código computacional utilizado. Como já mencionado, serão utilizados dois códigos computacionais distintos justamente para comprovar essa liberdade que a formulação contínua proporciona. Não está no escopo deste trabalho comparar as duas abordagens em nenhum aspecto portanto a partir deste ponto tudo relacionado ao Método Adjunto será única e exclusivamente relacionado à abordagem contínua. A derivação do Método Adjunto e suas respectivas condições de contorno para os propósitos desse trabalho estão exibidas no capítulo 3 . 


\section{O MÉTodo AduUnto}

Nesta seção será exibida a formulação do Método Adjunto, tanto da maneira mais geral, quanto a formulação específica para as equações Navier-Stokes caso incompressível.

\subsection{Formulação Geral}

A formulação apresentada a seguir segue a mesma linha de Cacuci et al. (CACUCI et al., 1980), porém de uma maneira mais resumida. Partindo-se de uma medida de mérito qualquer de um sistema físico conhecido, ou seja, um funcional que pode ser expresso da seguinte forma:

$$
I[\mathbf{X}, \mathbf{a}]=\int_{\Omega} F[\mathbf{X}(\rho), \mathbf{a}(\rho), \rho] \mathrm{d} \rho
$$

O vetor $\mathbf{X}$ representa as coordenadas do referido sistema no espaço de estados. O vetor $\rho$ expressa as coordenadas no espaço físico dos pontos pertencentes a um domínio de interesse $\Omega$. Esse domínio depende do tipo de aplicação para a qual a formulação será utilizada. Já o vetor a expressa o conjunto de parâmetros de controle do sistema. A escolha desses parâmetros também dependerá diretamente do tipo de aplicação desejada. Genericamente, escreve-se:

$$
\begin{aligned}
\mathbf{X}(\rho) & =\left[X_{1}(\rho), \ldots X_{k}(\rho)\right] \\
\rho & =\left(\rho_{1}, \ldots, \rho_{j}\right) \\
\mathbf{a}(\rho) & =\left[\mathbf{a}_{1}(\rho), \ldots \mathbf{a}_{i}(\rho)\right]
\end{aligned}
$$

Partindo para a variação do funcional, calcula-se a primeira derivada de Fréchet de (3.1), chegando, assim, na expressão:

$$
\delta I=\underbrace{\int_{\Omega} F_{X}^{\prime} \delta \mathbf{X}(\rho) \mathrm{d} \rho}_{\delta I_{X}}+\underbrace{\int_{\Omega} F_{\mathbf{a}}^{\prime} \delta \mathbf{a}(\rho) \mathrm{d} \rho}_{\delta I_{\mathbf{a}}}
$$


A variação total do funcional é expressa como soma de duas parcelas. A primeira, $\delta I_{X}$, representa a parcela física da variação total. A segunda diz respeito a variações paramétricas da aplicação sobre a qual o método será empregado. Normalmente, o termo $F_{\mathbf{a}}^{\prime} \delta \mathbf{a}(\rho)$ é conhecido e, portanto, sua variação $\delta I_{\mathbf{a}}$ pode ser obtida analiticamente.

Mas o termo $\delta I_{X}$ é, normalmente, desconhecido. Como forma de eliminá-lo da variação total, o método adjunto impõe as equações que governam a física do fenômeno no problema variacional como restrições ao mesmo. Assumindo-se que o operador $\mathbf{N}$ é composto por um número $K$ de EDPs ${ }^{1}$ não lineares, as quais, por sua vez, estão sujeitas a um conjunto de $\mathbf{B}$ condições de contorno, tem-se:

$$
\begin{aligned}
\mathbf{N}[\mathbf{X}(\rho), \mathbf{a}] & =\mathbf{Q}(\rho, \mathbf{a}) \\
\mathbf{B}[\mathbf{X}(\rho), \mathbf{a}]_{s} & =0
\end{aligned}
$$

O subscrito [ ] indica que as condições de contorno estão impostas nas fronteiras adequadas, ou seja cada condição de contorno na fronteira correspondente (condições de entrada na fronteira de entrada, condições de saída na fronteira de saída, etc.). O espaço físico possui dois produtos internos, um para o domínio e outro para a superfície, que são assumidos como sendo da seguinte forma:

$$
\begin{aligned}
\langle\Phi, \Psi\rangle & =\int_{\Omega} \Phi \cdot \Psi \mathrm{d} \Omega \\
\langle\Phi, \Psi\rangle_{S} & =\int_{S} \Phi \cdot \Psi \mathrm{d} \Omega
\end{aligned}
$$

Baseando-se nas equações exibidas até aqui, parte-se, agora, para o problema variacional já com as devidas restrições, oriundas das equações que governam a física do fenômeno. As restrições no problema variacional são impostas através de multiplicadores de Lagrange, que resulta na seguinte formulação para o funcional aumentado:

$$
\mathcal{J}(\mathbf{X}, \mathbf{a}, \Phi, \beta, \alpha)=I[\mathbf{X}, \mathbf{a}]-\langle\Phi, \mathbf{N}-\mathbf{Q}\rangle-\langle\beta, \mathbf{B}\rangle_{s}-\left\langle\alpha, \mathbf{a}-\mathbf{a}_{o}\right\rangle
$$

onde $\mathcal{J}$ é o funcional aumentado e $\Phi, \beta$ e $\alpha$ são multiplicadores de Lagrange. Do lado direito da equação há quatro termos. O primeiro é o funcional da equação (3.1), O segundo, com o multiplicador $\Phi$, impõe as equações governantes do escoamento, o terceiro impõe as condições de contorno, ao passo que o quarto termo assegura que os parâmetros de controle atendam a valores previamente

\footnotetext{
${ }^{1}$ Equações Diferenciais Parciais
} 
especificados na solução base, ou seja, $\mathbf{a}=\mathbf{a}_{o}$

Para determinar o(s) valor(es) crítico(s) de $\mathcal{J}$ é necessário conhecer a sua variação $\delta \mathcal{J}$. Partindo das equações (3.6) e (3.7), calculam-se suas derivadas de Fréchet:

$$
\begin{aligned}
\mathbf{L} \delta \mathbf{X} & =\mathbf{S} \delta \mathbf{a} \\
\mathbf{B}_{X}^{\prime} \delta \mathbf{X} & =-\mathbf{B}_{\mathbf{a}}^{\prime} \delta \mathbf{a}
\end{aligned}
$$

Acima foram introduzidos dois operadores, $\mathbf{L}$ e $\mathbf{S}$. O operador $\mathbf{L}$ representa a forma linearizada das equações governantes enquanto $\mathbf{S}$ carrega as variações dos parâmetros de controle. Tais operadores são definidos como:

$$
\begin{aligned}
& \mathbf{L} \equiv \mathbf{N}_{X}^{\prime} \\
& \mathbf{S} \equiv \mathbf{Q}_{\mathbf{a}}^{\prime}-\mathbf{N}_{\mathbf{a}}^{\prime}
\end{aligned}
$$

Prosseguindo com o cálculo das variações dos funcionais necessários para a determinação de $\delta \mathcal{J}$, calculam-se as derivadas de Fréchet dos segundo e terceiro termos da equação (3.10) e substitui-se a equação (3.14) no termo correspondente. Em seguida, faz-se uso do teorema de Gauss para transferir o operador diferencial do vetor de estado $\mathbf{X}$ para o multiplicador de Lagrange $\Phi$. Com esses passos chega-se a:

$$
\langle\Phi, \mathbf{L} \delta \mathbf{X}\rangle=\left\langle\mathbf{L}^{*} \Phi, \delta \mathbf{X}\right\rangle-P[\Phi, \delta \mathbf{X}]_{s}
$$

onde o termo $P[\Phi, \delta \mathbf{X}]_{s}$ é o concomitante bilinear, a integral de superfície gerada pela operação. Além disso, o operador $\mathbf{L}^{*}$ é o operador adjunto de $\mathbf{L}$, presente no primeiro termo do lado direito da equação 3.15 .

Calculando-se as derivadas de Fréchet dos funcionais restantes, lembrando que a derivada do funcional $I$ já foi calculada na equação (3.5), combinando as expressões obtidas acima, obtém-se a expressão da variação do funcional aumentado $\delta \mathcal{J}$, dada por:

$$
\begin{aligned}
\delta \mathcal{J}= & -\langle\delta \Phi, \mathbf{N}-\mathbf{Q}\rangle-\langle\delta \beta, \mathbf{B}\rangle_{s}-\left\langle\delta \alpha, \mathbf{a}-\mathbf{a}_{o}\right\rangle+\left\langle\mathbf{L}^{*} \Phi-F_{X}^{\prime}, \delta \mathbf{X}\right\rangle+ \\
& -\left\langle\beta, \mathbf{B}_{X}^{\prime} \delta X\right\rangle_{s}-\left[\left\langle P_{1}(\Phi), \mathbf{B}_{X}^{\prime} \delta \mathbf{X}\right\rangle_{s}+\left\langle\mathbf{B}^{*}(\Phi), \mathbf{A} \delta \mathbf{X}\right\rangle_{s}\right]+\left\langle\mathbf{F}_{\mathbf{a}}^{\prime}, \delta \mathbf{a}\right\rangle+ \\
& +\langle\Phi, \mathbf{S} \delta \mathbf{a}\rangle-\langle\alpha, \delta \mathbf{a}\rangle-\left\langle\beta, \mathbf{B}_{\mathbf{a}}^{\prime} \delta \mathbf{a}\right\rangle_{s}
\end{aligned}
$$

Nota-se que o concomitante bilinear presente em (3.15) foi escrito, na equação (3.16), na forma de uma soma de dois produtos internos, que estão entre colchetes. Ambos os termos, por apresentarem o subscrito $<>_{s}$, devem ser calculados sobre 
as fronteiras adequadas. Ainda sobre esses dois termos, nota-se que o primeiro envolve um $P_{1}(\Phi)$ e o operador de contorno linearizado $\mathbf{B}_{X}^{\prime} \delta \mathbf{X}$ enquanto o segundo envolve o operador de contorno adjunto $\mathbf{B}^{*}(\Phi)$ e o termo $\mathbf{A} \delta \mathbf{X}$.

A decomposição do concomitante bilinear $P$ não é única, bem como as definições de $P_{1}$ e A também não são. Na verdade, a única restrição que é imposta na formulação é a independência linear entre o operador $\mathbf{A}$ e $\mathbf{B}_{X}^{\prime}$.

Naturalmente, o funcional aumentado $\mathcal{J}$ atinge um valor crítico quando $\delta \mathcal{J}$ expresso na equação (3.16) vai a zero num extremo. Isto implica em variações arbitrárias, porém realizáveis, de seus parâmetros:

$$
\delta \mathcal{J}=0 \forall\{\delta \mathbf{X}, \delta \mathbf{a}, \delta \Phi, \delta \beta, \delta \alpha\} \in\{\text { lugar de realizabilidade }\}
$$

Porém, para que sentença acima seja verdadeira, algumas condições devem ser atendidas:

I.- As equações que regem o fenômeno físico (3.6) e suas respectivas condições de contorno (3.7) estão satisfeitas para a solução base. Adicionalmente, os parâmetros de controle devem ser idênticos aos valores prescritos para o problema, $\mathbf{a}=\mathbf{a}_{o}$. Essas condições implicam que os três primeiro termos de (3.16) são identicamente nulos para quaisquer variações dos multiplicadores de Lagrange.

II.- Ao impor a condição:

$$
\beta=-P_{1}(\Phi)
$$

a soma dos quinto e sexto termos de (3.16)será nula. Esta equação fornece, ainda, a solução de $\beta$ em função de $\Phi$.

III.- O vetor $\Phi$ deve satisfazer a equação adjunta, presente no quarto termo de (3.16), dada por:

$$
\mathbf{L}^{*} \Phi-F_{X}^{\prime}=0
$$

O operador a seguir, expresso no sétimo termo de (3.16) fornece as condições de contorno adjuntas:

$$
\mathbf{B}^{*}(\Phi)=0
$$

A equação acima (3.20) deve determinar o vetor $\Phi$ nos contornos, juntamente com $\beta$.

IV.- Reunindo-se os termos restantes da equação (3.16), o vetor $\alpha$ é especificado 
da seguinte forma:

$$
\langle\alpha, \delta \mathbf{a}\rangle=\left\langle\mathbf{F}_{\mathbf{a}}^{\prime}, \delta \mathbf{a}\right\rangle+\langle\Phi, \mathbf{S} \delta \mathbf{a}\rangle-\left\langle\beta, \mathbf{B}_{\mathbf{a}}^{\prime} \delta \mathbf{a}\right\rangle_{s}
$$

chegando $\delta \mathcal{J}=0$. Aliás, essa é a parte cabível ao gradiente de sensibilidade, $\delta I$.

Para comprovar a afirmativa feita no final do item acima a respeito do gradiente de sensibilidade (CACUCI et al., 1980), basta comprovar que as equações governantes (3.6) e (3.7) estão satisfeitas para uma variação $\Delta \mathcal{J}$ qualquer. Em seguida, para chegarmos ao conceito de gradiente de sensibilidade, parte-se da definição $\mathcal{J}$ dada pela equação (3.10), chegando-se a:

$$
\begin{aligned}
& \Delta \mathcal{J}=\Delta I-\langle\alpha, \Delta \mathbf{a}\rangle \\
& \text { onde }\left\{\begin{aligned}
\Delta \mathcal{J} & \equiv \mathcal{J}\left(X_{2}, \mathbf{a}_{2} ; \Phi_{2}, \beta_{2}, \alpha_{2}\right)-\mathcal{J}\left(X_{1}, \mathbf{a}_{1} ; \Phi_{1}, \beta_{1}, \alpha_{1}\right) \\
\Delta I & \equiv I\left(X_{2}, \mathbf{a}_{2}\right)-I\left(X_{1}, \mathbf{a}_{1}\right) \\
\Delta \alpha & \equiv \mathbf{a}_{2}-\mathbf{a}_{1}
\end{aligned}\right.
\end{aligned}
$$

onde 1 e 2 são diferentes configurações de parâmetros. Em particular, para uma variação infinitesimal $\Delta \mathcal{J} \rightarrow \delta \mathcal{J}$, sob as condições dadas e garantindo que $\Phi$, a e $\beta$ atendem aos itens I-IV, chega-se a um valor estacionário para $\mathcal{J}$. Por fim, é possível expressar:

$$
\begin{aligned}
\delta \mathcal{J} & =\delta I-\langle\alpha, \delta \mathbf{a}\rangle=0 \\
\delta I & =\langle\alpha, \delta \mathbf{a}\rangle \\
\delta I & =\left\langle\mathbf{F}_{\mathbf{a}}^{\prime}, \delta \mathbf{a}\right\rangle+\left\langle\Phi,\left(\mathbf{Q}_{\mathbf{a}}^{\prime}-\mathbf{N}_{\mathbf{a}}^{\prime}\right) \delta \alpha\right\rangle+\left\langle P_{1}(\Phi), \mathbf{B}_{\mathbf{a}}^{\prime} \delta \mathbf{a}\right\rangle_{s}
\end{aligned}
$$

que corresponde exatamente à equação (3.21), combinada com a equação (3.18) e a definição do operador $\mathbf{S}$, exibida na equação (3.14). A equação acima (3.23), nos permite calcular o gradiente de sensibilidade com base na solução adjunta dada pelo vetor $\Phi$ em relação a variações dos parâmetros de controle $\delta$ a, individualmente.

Vale ressaltar que todas as variações que dizem respeito à física do fenômeno, $\delta \mathbf{X}$ foram removidas da expressão do gradiente de sensibilidade. Analisando a equação (3.23), o primeiro termo do lado direito é exatamente $\delta I_{\mathbf{a}}$, o segundo calcula os efeitos das variações dos parâmetros $\delta \alpha$ nas equações governantes e, por fim, o terceiro é diretamente relacionado às condições de contorno do problema. 


\subsection{Formulação Para as Equações de Navier-Stokes, Caso Incompressível}

Após formular o método Adjunto de sua forma mais geral, nesta seção, serão feitas as derivações do método para a aplicação específica das equações de NavierStokes, juntamente com suas respectivas condições de contorno.

A derivação aqui apresentada seguirá basicamente a metodologia empregada por Jameson (NADARAJAH; JAMESON, 2007; JAMESON; MARTINELLI; PIERCE, 1998). Esta metodologia apresenta diferenças da proposta por Cacuci et al. (CACUCI et al., 1980), na qual as condições de contorno do escoamento não estão impostas explicitamente no problema variacional. Há diferenças também no modo de expressar o funcional aumentado, descrito na seção (3.1). Outros detalhes dessas diferenças serão abordados mais adiante.

\subsubsection{Considerações Preliminares}

O objetivo do emprego do método Adjunto para as equações de Navier Stokes é encontrar quais configurações do escoamento acarretarão em um valor extremo de uma determinada medida de mérito, que dependerá única e exclusivamente da aplicação desejada. Tal medida de mérito é normalmente um funcional das variáveis do escoamento. Pode ser uma integral sobre o domínio do escoamento $(\mathcal{D})$, uma integral de superfície sobre alguma fronteira ou até mesmo de um corpo.

Para exemplificar, partir-se-á de um funcional da força exercida pelo fluido em um corpo em uma dada direção, funcional esse expresso por:

$$
I=\frac{1}{T} \int_{0}^{T} \oint_{B} G(\mathbf{n} \cdot \boldsymbol{\sigma} \cdot \mathbf{e}) \mathrm{d} S \mathrm{~d} t
$$

onde $\boldsymbol{\sigma}$ é o tensor das tensões, $\boldsymbol{\sigma}=\boldsymbol{\tau}-p \mathbf{I}$, e os vetores $\mathbf{n}$ e e representam a normal unitária à superfície do corpo e a direção de projeção da força, respectivamente. Além disso, assume-se que $G$ é uma função bem comportada, ou seja, contínua e limitada em todos os pontos do domínio $\Omega$ e com derivadas também contínuas em todo o domínio.

Em se tratando de escoamentos incompressíveis e isotérmicos, o estado do sistema é completamente caracterizado em termos dos campos de velocidade e pressão $(\mathbf{u}, p)$. Esses campos descrevem um espaço de estados e, consequente- 
mente, definem um vetor de estados dado por:

$$
\mathbf{q} \equiv\left(u^{1}, u^{2}, u^{3}, p\right)^{T} \Rightarrow\left\{\begin{array}{l}
q^{i}=u^{i} \text { para } i=1,2,3 \\
q^{4}=p
\end{array}\right.
$$

O espaço físico é representado por $\left(x^{i^{\prime}}, t\right)$ em coordenadas cartesianas. O domínio do escoamento $\mathcal{D}$ e o tempo $T$ definem o domínio completo do problema $\Omega=$ $\mathcal{D} \times(0, T)$.

Há diversas aplicações possíveis para o método Adjunto nesse caso. Uma delas, consiste em encontrar uma configuração de geometria que acarretaria um valor mínimo do funcional (3.24), sob certas condições do escoamento em torno do corpo. Para essa aplicação, assume-se que a geometria do corpo é descrita por um conjunto finito de parâmetros.

$$
S\left(x^{i^{\prime}} ; a^{k}\right) \Leftrightarrow S\left(\xi^{j} ; a^{k}\right)
$$

A geometria do corpo pode ser descrita em coordenadas cartesianas $\left(x^{i^{\prime}}\right)$ ou generalizadas $\left(\xi^{j}\right)$. Para distinção de ambas, denominar-se-á a descrição cartesiana por $S^{\prime}$ e a generalizada por $S$

Como mencionado, deseja-se minimizar o funcional $I$. Estabelece-se que $a^{k}$ são os parâmetros de controle do problema e o funcional $I$ é a medida de mérito correspondente a tais parâmetros. Com isso, é de grande utilidade saber como essa medida $I$ se comporta face a variações desses. Em outras palavras, é de grande valia saber a sensibilidade de $I_{o}$ em relação aos parâmetros de controle. Essa sensibilidade é calculada por meio do, já citado, gradiente de sensibilidade $\partial I / \partial a^{k}$.

Entretanto tanto a integral em (3.24) quanto a física do escoamento sofrem alterações sob qualquer variação de $a^{k}$. Neste momento aparece a necessidade de separação das variações físicas e geométricas. Uma maneira de realizar essa separação é definindo uma transformação de coordendas $\left(x^{i^{\prime}} \rightleftharpoons \xi^{j}\right)$, na qual o corpo é descrito sobre um plano coordenado constante. Tal mudança pode ser vista, de forma esquemática em A.1.

Nessa mudança. a representação geométrica da superfície do corpo no espaço transformada é fixa. Isso implica que ela permanece inalterada para quaisquer variações dos parâmetros de controle $\delta a^{k}$, mesmo que haja mudanças físicas na forma. Somente os operadores de transformação e os termos da métrica $\beta$ e $\beta^{-1}$ serão alterados com $\delta a^{k}$ bem como a métrica do espaço transformado. Dessa maneira, variações físicas e geométrica estão devidamente separadas. Além de 
permitir uma melhor análise das variações de $I_{o}$, a mudança descrita facilita o problema variacional, pois o domínio de integração permanece fixo no espaço transformado (GELFAND; FOMIN, 1963). Partindo do vetor de velocidades descrito em (A.2)

$$
\begin{aligned}
u^{i} & =\beta_{j^{\prime}}^{i} u^{j^{\prime}} \\
\delta u^{i} & =\underbrace{\beta_{j^{\prime}}^{i} \delta u^{j^{\prime}}}_{\left(\delta u^{i}\right)_{T}}+\underbrace{\left.\delta u^{i}\right)_{P}}+\left(\delta u^{i}\right)_{M}
\end{aligned}
$$

A variação total $\left(\delta u^{i}\right)_{T}$ é expressa como soma de dois termos. O primeiro representa a parcela física da variação para uma transformação fixa ao passo que o segundo corresponde à variação geométrica no espaço físico sob dadas condições fixas do escoamento. Para diferenciar variações físicas e geométricas, serão utilizados os índices ()$_{P}$ and ()$_{M}$, respectivamente, quando necessários.

Voltando à expressão da medida de mérito $I_{o}$, é necessário escrever a equação (3.24) no espaço transformado e, em seguida, calcular o gradiente de sensibilidade:

$$
\begin{aligned}
I & =\frac{1}{T} \int_{0} \oint_{B} G\left|\frac{d S^{\prime}}{d S}\right| \mathrm{d} S \mathrm{~d} t \\
\frac{\partial I}{\partial a^{k}} & =\frac{1}{T} \int_{0}^{T} \oint_{B}\left[\frac{\partial G}{\partial q^{i}} \frac{\partial q^{i}}{\partial a^{k}}\left|\frac{d S^{\prime}}{d S}\right|+G \frac{\partial}{\partial a^{k}}\left|\frac{d S^{\prime}}{d S}\right|\right] \mathrm{d} S \mathrm{~d} t
\end{aligned}
$$

O termo $\left|\mathrm{d} S^{\prime} / \mathrm{d} S\right|$ representa uma razão entre elementos de área no espaço cartesiano e generalizado. Sua derivação se encontra no apêndice B.

O primeiro termo dentro da integral inferior diz respeito à sensibilidade da física do escoamento em relação aos parâmetros de controle. O segundo termo é uma medida de como a geometria do corpo se altera em resposta a mudanças nos parâmetros de controle. Dessa forma fica claro que a mudança proposta anteriormente separa variações físicas e geométricas. Adicionalmente, os termos $\left(\partial G / \partial q^{i}\right)$ and $\left(\partial\left|d S^{\prime} / d S\right| / \partial a^{k}\right)$ são conhecidos graças às expressões de $G$ and $S^{\prime}$.

O desafio relativo à equação (3.28) consiste no termo que diz respeito à variação física $\left(\partial q^{i} / \partial a^{k}\right)$, o qual muito raramente é conhecido. Este é o termo que faz com que diversas simulações do escoamento sejam necessárias, uma para cada variação de parâmetro de controle $\delta a^{k}$, tomada separadamente. Isso acarreta a necessidade, a princípio, de restringir tais variações a um espaço limitado de soluções possíveis. 


\subsubsection{Equações Adjuntas}

Para um escoamento incompressível e isotérmico, as equações de NavierStokes são as exibidas abaixo.

$$
\left\{\begin{aligned}
\partial_{o} \mathbf{u}+(\mathbf{u} \cdot \nabla) \mathbf{u}+v \nabla p-\nu \nabla^{2} \mathbf{u} & =0 \\
\nabla \cdot \mathbf{u} & =0
\end{aligned}\right.
$$

Acima, u é o campo de velocidades do escoamento; $p$, a pressão; $v$, o volume específico do fluido e $\nu$, a viscosidade cinemática. Na derivação a seguir, admitese que as propriedades do fluido são constantes. Neste trabalho sempre que houver referência às equações de Navier-Stokes, estarão sendo consideradas as equações de conservação da quantidade de movimento e da continuidade.

Como já visto, as equações de Navier-Stokes funcionam como restrições não holonômicas no problema variacional (GELFAND; FOMIN, 1963). Isso significa que, essas restrições não limitam o domínio do problema mas sim o domínio das variações dos parâmetros. Além disso, uma restrição não holonômica consiste em equações não integráveis que envolvam os parâmetros do problema e suas respectivas derivadas.

Com essa imposição, o funcional aumentado passa a ser expresso da seguinte maneira:

$$
\begin{aligned}
\mathcal{J} & =\frac{1}{T} \int_{0} \oint_{B} G(\mathbf{n} \cdot \boldsymbol{\sigma} \cdot \mathbf{l})\left|\frac{d S^{\prime}}{d S}\right| \mathrm{d} S \mathrm{~d} t+\frac{1}{T}\left\{\int_{\Omega} \theta \nabla \cdot \mathbf{u} \mathrm{d} \Omega+\right. \\
& \left.-\int_{\Omega} \boldsymbol{\psi} \cdot\left[\partial_{o} \mathbf{u}+(\mathbf{u} \cdot \nabla) \mathbf{u}+v \nabla p-\nu \nabla^{2} \mathbf{u}\right] \mathrm{d} \Omega\right\}
\end{aligned}
$$

onde $\theta$ e $\psi$ são multiplicadores de Lagrange, cujos sinais foram escolhidos por conveniência algébrica. É conveniente definir uma notação para uma integração sobre o domínio do escoamento $\Omega$. A integração obedece a seguinte notação:

$$
\int_{\Omega} f\left(x^{j^{\prime}}, t\right) \mathrm{d} \Omega \equiv \int_{0}^{T} \int_{\mathcal{D}} f\left(x^{j^{\prime}}, t\right) \mathrm{d} V \mathrm{~d} t=\int_{0}^{T} \int_{\mathcal{D}} f\left(\xi^{k}, t\right) J \mathrm{~d} \xi^{1} \mathrm{~d} \xi^{2} \mathrm{~d} \xi^{3} \mathrm{~d} t
$$

O símbolo $J$ corresponde ao Jacobiano da transformação de coordenadas do sistema cartesiano para o sistema descrito por coordenadas generalizadas (A.2).

Adotando o mesmo procedimento usado na equação (3.28) com a equação (3.30) é necessário calcular a primeira derivada do funcional aumentado $I \mathrm{em}$ função de variações físicas e geométricas. Porém, nesta etapa, isso acarreta que a 
mesma avaliação seja feita, também, para as equações de Navier-Stokes. No espaço de estados, é possível escrever essas equações na forma de operadores matriciais como $\mathbf{N q}=0$, onde $\mathbf{N}$ é definido da seguinte forma (BARKLEY; BLACKBURN; SHERWIN, 2002):

$$
\left(\begin{array}{c|c}
\partial_{o}+(\mathbf{u} \cdot \nabla)-\nu \nabla^{2} & v \nabla \\
\hline \nabla \cdot & 0
\end{array}\right)\left(\begin{array}{c}
\mathbf{u} \\
\hline p
\end{array}\right)=\left(\begin{array}{c}
0 \\
\hline 0
\end{array}\right)
$$

Define-se um vetor de variáveis de estado, contínuo e diferenciável até, no mínimo, $2^{\mathrm{a}}$ ordem em todo o domínio, da forma $\mathbf{q}=(\mathbf{u}, p)^{T}$. Assim, utiliza-se a forma simplificada da derivada de Fréchet para calcular as primeira derivadas do vetor de estado (CACUCI et al., 1980; LUSTERNICK; SOBOLEV, 1961).

$$
\mathbf{N}_{, q} \delta \mathbf{q}+\mathbf{N}_{, a} \delta \mathbf{a}=\frac{\partial}{\partial \varepsilon_{1}}\left[\mathbf{N}\left(\mathbf{q}+\varepsilon_{1} \delta \mathbf{q}\right)\right]_{\varepsilon_{1}=0}+\frac{\partial}{\partial \varepsilon_{2}}\left[\mathbf{N}\left(\mathbf{a}+\varepsilon_{2} \delta \mathbf{a}\right)\right]_{\varepsilon_{2}=0}=0
$$

Baseando-se no conjunto completo de equações (A.39), é simples calcular a primeira derivada em relação às variáveis de estado $\delta \mathbf{q}$. Conhecendo-se as expressões para derivadas covariantes disponíveis no apêndice A e considerando todos os termos da métrica como sendo constantes, a derivação prossegue da seguinte forma:

$$
\mathbf{N}_{, q} \delta \mathbf{q}=\left(\begin{array}{c}
\partial_{o} \delta u^{i}+\left.\left[u^{i} \delta u^{j}+u^{j} \delta u^{i}+g^{i j} v \delta p-\nu\left(\left.\delta u^{i}\right|^{j}+\left.\delta u^{j}\right|^{i}\right)\right]\right|_{j} \\
\left.\delta u^{i}\right|_{i}
\end{array}\right)
$$

Ressalta-se que o tensor das tensões completo (A.37) foi considerado na variação física da equação do momento. Adiante, ficarão claras as razões para tal procedimento.

$$
v \delta \tau^{i j}=\nu\left(\left.\delta u^{i}\right|^{j}+\left.\delta u^{j}\right|^{i}\right)
$$

No apêndice A é mostrado que ambas as formas completa (A.39) e reduzida (A.40) da equação de Navier-Stokes generalizadas são equivalentes fisicamente. Porém, a última se apresenta de uma forma mais simples no que diz respeito aos termos relativos à métrica, o que facilita o cálculo da variações de tais termos. Faz-se a derivação mantendo todos os parâmetros físicos como constantes:

$$
\mathbf{N}_{, a} \delta a=\left(\begin{array}{c}
\partial_{o}\left(\delta J u^{r^{\prime}}\right)+\left[\delta\left(J \beta_{n^{\prime}}^{j}\right)\left(u^{r^{\prime}} u^{n^{\prime}}+g^{r^{\prime} n^{\prime}} p v-\nu u_{, p^{\prime}}^{r^{\prime}} g^{p^{\prime} n^{\prime}}\right)\right]_{, j} \\
{\left[\delta\left(J \beta_{q^{\prime}}^{k}\right) u^{q^{\prime}}\right]_{, k}}
\end{array}\right)
$$

A primeira derivada do funcional aumentado (3.30) é obtida combinando as 
equações (3.28), (3.34) e (3.36):

$$
\begin{aligned}
& T \delta I=\underbrace{\int_{0} \oint_{B}\left(\frac{\partial G}{\partial p} \delta p+\frac{\partial G}{\partial \mathcal{F}^{k}} \delta \mathcal{F}^{k}\right)\left|\frac{d S^{\prime}}{d S}\right| \mathrm{d} S \mathrm{~d} t}_{I_{1}}+\underbrace{\left.\int_{\Omega} \theta \delta u^{k}\right|_{k} \mathrm{~d} \Omega}_{I_{2}}+ \\
& -\underbrace{\int_{\Omega} \psi_{i}\left\{\partial_{o} \delta u^{i}+\left.\left[u^{i} \delta u^{j}+u^{j} \delta u^{i}+g^{i j} v \delta p-\nu\left(\left.\delta u^{i}\right|^{j}+\left.\delta u^{j}\right|^{i}\right)\right]\right|_{j}\right\} \mathrm{d} \Omega}_{I_{3}}+ \\
& +\underbrace{\int_{0}^{T} \oint_{B} G \delta\left|\frac{d S^{\prime}}{d S}\right| \mathrm{d} S \mathrm{~d} t}_{I_{4}}+\underbrace{\int_{\Omega} \theta\left[\delta\left(J \beta_{q^{\prime}}^{k}\right) u^{q^{\prime}}\right]_{, k} \mathrm{~d} \Omega}_{I_{5}}+ \\
& -\underbrace{\int_{\Omega} \psi_{r^{\prime}}\left\{\partial_{o}\left(\delta J u^{r^{\prime}}\right)+\left[\delta\left(J \beta_{n^{\prime}}^{j}\right)\left(u^{r^{\prime}} u^{n^{\prime}}+g^{r^{\prime} n^{\prime}} p v-\nu u_{, p^{\prime}}^{r^{\prime}} g^{p^{\prime} n^{\prime}}\right)\right]\right.}_{I_{6}}\} \mathrm{d} \Omega
\end{aligned}
$$

O símbolo $\mathcal{F}^{k}$ que aparece na integral $I_{1}$ denota os componentes da força resultantes que o fluido exerce sobre uma superfície, cuja normal é $\mathbf{n}$, expressa por: $\mathcal{F}^{k}=\tau^{k j} n_{j} \Rightarrow \delta \mathcal{F}^{k}=\delta \tau^{k j} n_{j}$. Isso nada mais é do que uma outra maneira de expressar $\partial G / \partial q^{i}$ para $1 \leq i \leq 3$. O passo mais importante na derivação matemática que pode ser feito na equação (3.37), como já mencionado, é a divisão das seis integrais em dois blocos distintos. O primeiro bloco contém as integrais que levam em conta apenas variações físicas $\left(\left\{I_{1}, I_{2}, I_{3}\right\}\right)$ ao passo que o segundo bloco contém as integrais que dizem respeito à variações métricas $\left(\left\{I_{4}, I_{5}, I_{6}\right\}\right)$. O segundo bloco é mantido na sua forma original, como parte do cálculo do gradiente de sensibilidade enquanto o primeiro está diretamente ligado ao problema adjunto.

A idéia central no método adjunto consiste em eliminar da expressaão do gradiente de sensibilidade, os termos correspondentes à variações físicas. Isso é feito por meio do Teorema de Gauss (A.41). O procedimento visa transferir operadores que originalmente agem diretamente sobre as variáveis do escoamento para as variáveis adjuntas, como aparece nas equações (3.15) e (3.16). Isso só é possível admitindo-se que todas as variáveis físicas são contínuas e diferenciáveis no interior do domínio $\Omega$, o que é uma hipótese razoável considerando-se campos de velocidade e pressão, dadas as condições do escoamento aqui estabelecidas.

Em relação aos termos da equação (3.37) sobre os quais será aplicado o Teorema de Gauss, é conveniente analisá-los separadamente. A primeira integral, $I_{1}$, é calculada sobre a superfície do corpo e em um intervalo de tempo, e perma- 
necerá rigorosamente desta forma. Em $I_{2}$ tem-se a primeira integral de volume. Para tratá-la, juntamente com as integrais $I_{3}, I_{5}$ e $I_{6}$, é definido o elemento de volume no espaço transformado da seguinte forma, baseado na equação (3.31):

$$
\mathrm{d} \Omega=\mathrm{d} V \mathrm{~d} t=J \mathrm{~d} \xi^{1} \mathrm{~d} \xi^{2} \mathrm{~d} \xi^{3} \mathrm{~d} t \equiv J \mathrm{~d} \bar{\xi} \mathrm{d} t
$$

onde o símbolo $\mathrm{d} \bar{\xi}$ representa o elemento de volume no espaço transformado. A integral $I_{2}$ pode ser escrita na forma:

$$
\left.\int_{\Omega} \Theta \delta u^{k}\right|_{k} \mathrm{~d} \bar{\xi} \mathrm{d} t=\int_{0 \partial \mathcal{D}}^{T} \oint_{\Omega} \Theta \delta u^{k} n_{k} \mathrm{~d} S \mathrm{~d} t-\left.\int_{\Omega} \delta u^{k} \Theta\right|_{k} \mathrm{~d} \bar{\xi} \mathrm{d} t
$$

onde definiu-se $\Theta \equiv J \theta$.

O primeiro termo em $I_{3}$ corresponde a uma derivada temporal. A idéia aqui é inverter a ordem de integração ao invés de utilizar o Teorema de Gauss. Isso é feito admitindo que as variáveis do escoamento são contínuas e diferenciáveis no interior do domínio $\Omega$

$$
\int_{\Omega} \varphi_{i} \partial_{o} \delta u^{i} \mathrm{~d} \bar{\xi} \mathrm{d} t=\left[\int_{\mathcal{D}} \varphi_{i} \delta u^{i} \mathrm{~d} \bar{\xi}\right]_{0}^{T}-\int_{\Omega} \delta u^{i} \partial_{o} \varphi_{i} \mathrm{~d} \bar{\xi} \mathrm{d} t
$$

onde foi definido $\varphi_{i} \equiv J \psi_{i}$.

Os segundo e terceiro termos do funcional $I_{3}$ serão tratados conjuntamente. Chama-se, por conveniência essa integral de $\Delta_{c}$. Utilizando a equação da continuidade presente na segunda linha de (3.34) e (A.39), pode-se escrever:

$$
\begin{aligned}
& \Delta_{c}=\left.\int_{\Omega} \varphi_{i}\left(u^{j} \delta u^{i}+u^{i} \delta u^{j}\right)\right|_{j} \mathrm{~d} \Omega=\int_{\Omega} \varphi_{i}\left(\left.u^{j} \delta u^{i}\right|_{j}+\left.u^{i}\right|_{j} \delta u^{j}+\left.u^{i} \delta u^{j}\right|_{j}\right) \mathrm{d} \bar{\xi} \mathrm{d} t \\
&= \int_{0 \partial \mathcal{D}}^{T} \oint_{i} \varphi_{i} u^{j} \delta u^{i} n_{j} \mathrm{~d} S \mathrm{~d} t-\left.\int_{\Omega} u^{j} \delta u^{i} \varphi_{i}\right|_{j} \mathrm{~d} \bar{\xi} \mathrm{d} t+\int_{\Omega} \varphi_{i}\left(\left.u^{i}\right|_{j} \delta u^{j}+\left.u^{i} \delta u^{j}\right|_{j}\right) \mathrm{d} \bar{\xi} \mathrm{d} t \\
&= \int_{0 \partial \mathcal{D}}^{T} \oint_{i} u^{j} \delta u^{i} n_{j} \mathrm{~d} S \mathrm{~d} t-\int_{\Omega} \delta u^{i}\left(\left.u^{j} \varphi_{i}\right|_{j}-\left.\varphi_{j} u^{j}\right|_{i}\right) \mathrm{d} \bar{\xi} \mathrm{d} t \\
&-\int_{\Omega} \varphi_{i} u^{i}\left[\delta\left(J \beta_{q^{\prime}}^{k}\right) u^{q^{\prime}}\right]_{, k} \mathrm{~d} \bar{\xi} \mathrm{d} t
\end{aligned}
$$

Na derivação acima foi realizada integração por partes somente no primeiro termo 
de $\Delta_{c}$. Tratando, agora, o quarto termo do funcional $I_{3}$

$$
\int_{\Omega} \varphi_{i}\left(g^{i j} v \delta p\right) \mid{ }_{j} \mathrm{~d} \bar{\xi} \mathrm{d} t=\int_{0 \partial \mathcal{D}}^{T} \oint_{\Omega} v \delta p \varphi^{j} n_{j} \mathrm{~d} S \mathrm{~d} t-\int_{\Omega} v \delta p \varphi^{j}{ }_{j} \mathrm{~d} \bar{\xi} \mathrm{d} t
$$

Na passagem acima, utilizou-se a propriedade de que a derivada covariante do tensor métrico é identicamente nula (A.24).

Em seguida é feito o tratamento nos dois últimos termos de $I_{3}$. Aqui, serão necessárias duas integrações por partes. Integrando sem a constante $\nu$, obtem-se:

$$
\begin{aligned}
\left.\int_{\Omega} \varphi_{i}\left(\left.\delta u^{i}\right|^{j}+\left.\delta u^{j}\right|^{i}\right)\right|_{j} \mathrm{~d} \bar{\xi} \mathrm{d} t & =\int_{0 \partial \mathcal{D}}^{T} \varphi_{i}\left(\left.\delta u^{i}\right|^{j}+\left.\delta u^{j}\right|^{i}\right) n_{j} \mathrm{~d} S \mathrm{~d} t+ \\
& -\left.\int_{\Omega} \varphi_{i}\right|_{j}\left(\left.\delta u^{i}\right|^{j}+\left.\delta u^{j}\right|^{i}\right) \mathrm{d} \bar{\xi} \mathrm{d} t \\
& =\int_{0 \partial \mathcal{D}}\left[\varphi_{i}\left(\left.\delta u^{i}\right|^{j}+\left.\delta u^{j}\right|^{i}\right) n_{j}+\right. \\
& +\int_{\Omega}\left(\left.\left.\delta u^{i} \varphi_{i}\right|_{j}\right|^{j}+\left.\left.\delta u^{j} \varphi_{i}\right|_{j}\right|^{i}\right) \mathrm{d} \bar{\xi} \mathrm{d} t
\end{aligned}
$$

Como $\nu$ é constante, multiplica-se o mesmo pela equação (3.43). Com isso, aparece o termo de variação $\delta \tau^{i j}$ presente na equação (3.35). Fazendo $\left.\varphi^{i}\right|_{i}=$ $\left.\left(\varphi_{j} g^{j i}\right)\right|_{i}=\left.\varphi_{j}\right|^{j}$, chega-se a:

$\int_{\Omega} \frac{\left.\varphi_{i} \delta \tau^{i j}\right|_{j}}{\rho} \mathrm{d} \bar{\xi} \mathrm{d} t=\int_{0 \partial \mathcal{D}}^{T} \oint_{\varphi_{i} \delta \mathcal{F}^{i}}-\nu \delta u^{i}\left(\left.\varphi_{i}\right|_{j}+\left.\varphi_{j}\right|_{i}\right) n^{j} \mathrm{~d} S \mathrm{~d} t+\left.\int_{\Omega} \delta u^{i} \nu\left(\left.\varphi_{i}\right|^{j}+\left.\varphi^{j}\right|_{i}\right)\right|_{j} \mathrm{~d} \bar{\xi} \mathrm{d} t$

chegando a uma forma simétrica e concisa. A equação (3.44) esclarece porque a expressão $\delta \tau^{i j}$ foi mantida na sua forma completa na variação física das Equações de Navier Stokes (3.34)-(3.35). O objetivo era obter essa variação novamente como uma integral de superfície contendo o termo $\delta \mathcal{F}^{i}=\delta \tau^{i j} n_{j}$.

Vale ressaltar que as integrais de superfície presentes nas equações (3.39), (3.41)-(3.44) e o termo dentro de colchetes da equação (3.40), compõem o concomitante bilinear que, posteriormente, dará origem ao problema de contorno adjunto. Por fim, substituindo os resultados obtidos em (3.39)-(3.44) na equação 
(3.37), obtem-se:

$$
\begin{aligned}
T \delta I & =\int_{\Omega}\left\{\left.\varphi^{j}\right|_{j} v \delta p+\left[\partial_{o} \varphi_{i}+\left.u^{j} \varphi_{i}\right|_{j}-\left.\varphi_{j} u^{j}\right|_{i}-\left.\Theta\right|_{i}+\left.\nu\left(\left.\varphi_{i}\right|^{j}+\left.\varphi^{j}\right|_{i}\right)\right|_{j}\right] \delta u^{i}\right\} \mathrm{d} \bar{\xi} \mathrm{d} t+ \\
& -\int_{0 \partial \mathcal{D}}^{T}\left\{\left[u^{j} \varphi_{i} n_{j}-\Theta n_{i}+\nu\left(\left.\varphi_{i}\right|_{j}+\left.\varphi_{j}\right|_{i}\right) n^{j}\right] \delta u^{i}+v\left(\delta p n^{i}-\delta \mathcal{F}^{i}\right) \varphi_{i}\right\} \mathrm{d} S \mathrm{~d} t+ \\
& \left.-\int_{\mathcal{D}} \varphi_{i} \delta u^{i} \mathrm{~d} V\right]_{0}^{T}+\int_{0} \oint_{B}\left[\frac{\partial G}{\partial p} \delta p+\frac{\partial G}{\partial \mathcal{F}^{k}} \delta \mathcal{F}^{k}\right]\left|\frac{\mathrm{d} S^{\prime}}{\mathrm{d} S}\right| \mathrm{d} S \mathrm{~d} t+ \\
& +\int_{0}^{T} \oint_{B} G \delta\left|\frac{\mathrm{d} S^{\prime}}{\mathrm{d} S}\right| \mathrm{d} S \mathrm{~d} t+\int_{\Omega}\left(\frac{\Theta}{J}-\varphi_{i} u^{i}\right)\left[\delta\left(J \beta_{q^{\prime}}^{k}\right) u^{q^{\prime}}\right]_{, k} \mathrm{~d} \Omega+ \\
& -\int_{\Omega} \psi_{r^{\prime}}\left\{\partial_{o}\left(\delta J u^{r^{\prime}}\right)+\left[\delta\left(J \beta_{n^{\prime}}^{j}\right)\left(u^{r^{\prime}} u^{n^{\prime}}+g^{r^{\prime} n^{\prime}} p v-\nu u_{, p^{\prime}}^{r^{\prime}} g^{p^{\prime} n^{\prime}}\right)\right]_{, j}\right\} \mathrm{d} \Omega
\end{aligned}
$$

Todas as variações físicas presentes na equação (3.45) são possíveis de ocorrer uma vez que satisfazem as equações de Navier-Stokes. Porém, apesar disso, são arbitrárias.

Como já comentado neste capítulo, a construção do problema adjunto consiste em eliminar as variações físicas da expressão para o cálculo do gradiente de sensibilidade. Na equação (3.45), esses termos correspondem às quatro primeiras integrais. A derivação a seguir fará com que isso aconteça.

A primeira integral da equação (3.45) é feita ao longo de todo o domínio $\Omega$ e envolve duas variações arbitrárias independentes, $\delta p$ e $\delta \mathbf{u}$. Para que essa integral se anule, é necessário que os termos que multiplicam essas variações sejam nulos. Para que isso ocorra, as variáveis adjuntas $\varphi$ e $\Theta$ devem satisfazer as seguintes condições:

$$
\left\{\begin{array}{r}
\left.\varphi^{j}\right|_{j}=0 \\
\underline{\partial_{o} \varphi_{i}}+\left.u^{j} \varphi_{i}\right|_{j}-\left.\varphi_{j} u^{j}\right|_{i}-\left.\Theta\right|_{i}+\underline{\left.\left.\nu \varphi_{i}\right|^{j}\right|_{j}}=0
\end{array}\right.
$$

onde o último termo do lado esquerdo da equação da segunda equação foi simplificado em relação à sua forma original em (3.45). Além disso, a derivada temporal e o termo difusivo da segunda equação estão sublinhados pois tem o mesmo sinal. Isso implica que, posteriormente não se comportará como mecanismo difusivo e sim como um termo amplificador de perturbações na solução adjunta. Isso faz com que as EDPs se tornem extremamente instáveis (BARKLEY; BLACKBURN; SHERWIN, 2002). 
A solução para esse problema é bem difundida na literatura (BARKLEY; BLACKBURN; SHERWIN, 2002), e consiste em inverter a integração temporal. As equações são integradas de um estado final conhecido no sentido contrário do tempo a até um estado inicial desconhecido. Esse procedimento é consistente com as condições de tempo adjuntas, conforme será mostrado adiante. A integração no sentido contrário é feita definindo uma variável adjunta de tempo:

$$
t^{*} \equiv T-t \Rightarrow \mathrm{d} t^{*}=-\mathrm{d} t \quad ; \quad\left\{\begin{array}{l}
t=0 \Rightarrow t^{*}=T \\
t=T \Rightarrow t^{*}=0
\end{array}\right.
$$

com uma notação compacta correspondente: $\partial_{o *} \equiv \partial() / \partial t^{*}$.

Substituindo essa definição na derivada correspondente na equação (3.46):

$$
\left\{\begin{array}{r}
\left.\varphi^{j}\right|_{j}=0 \\
\partial_{o *} \varphi_{i}-\left.u^{j} \varphi_{i}\right|_{j}+\left.\varphi_{j} u^{j}\right|_{i}+\left.\Theta\right|_{i}-\left.\left.\nu \varphi_{i}\right|^{j}\right|_{j}=0
\end{array}\right.
$$

Essas EDPs respresentam as equações adjuntas de Navier-Stokes adjuntas aplicadas a escoamentos isotérmicos e incompressíveis. Observa-se que a equação (3.48) é linear, uma vez que todos os coeficientes nas EDPs são claramente independentes das variáveis adjuntas $(\boldsymbol{\varphi}, \Theta)$.

Comparando a equação (3.48) com a forma original das equação de NavierStokes (A.39) é perceptível que ambas apresentam uma estrutura similar. Isso é ainda mais evidente se a equação adjunta for comparada com a forma linearizada da equação de Navier-Stokes (3.34). A diferença ocorre nos termos convectivos. Apesar disso, a derivada temporal e o termo convectivo apresentam similaridades entre si e esses termos que determinarão a característica da EDP (CARRIER; PEARSON, 1988).

O conjunto de equações de Navier-Stokes (A.5)-(A.6), as equações de energia e momento são parabólicas no domínio espaço-temporal e tornam-se elípticas para escoamentos em regime permanente, enquanto a continuidade é hiperbólica (HIRSCH, 1994a). Esse caráter híbrido apresentado pelas equações permanece o mesmo para escoamentos incompressíveis e, devido à similaridade das equações, o conjunto de equações adjuntos exibido na equação (3.48) apresentará o mesmo comportamento. Tal característica define a natureza matemática do problema de contorno (MORSE; FESHBACH, 1953a; MORSE; FESHBACH, 1953b). E, de outro lado, a física do problema de contorno aparecem nas segunda, terceira e quarta integrais presentes na equação (3.45). 


\subsubsection{Condições de Contorno}

As condições de contorno adjuntas são concernentes às segunda e quarta integrais da equação (3.45), novamente aqui reproduzidas:

$$
\begin{aligned}
& -\int_{0 \partial \mathcal{D}} \oint_{0}\left\{\left[u^{j} \varphi_{i} n_{j}-\Theta n_{i}+\nu\left(\left.\varphi_{i}\right|_{j}+\left.\varphi_{j}\right|_{i}\right) n^{j}\right] \delta u^{i}+v\left(\delta p n^{i}-\delta \mathcal{F}^{i}\right) \varphi_{i}\right\} \mathrm{d} S \mathrm{~d} t+ \\
& +\int_{0} \oint_{B}\left[\frac{\partial G}{\partial p} \delta p+\frac{\partial G}{\partial \mathcal{F}^{k}} \delta \mathcal{F}^{k}\right]\left|\frac{\mathrm{d} S^{\prime}}{\mathrm{d} S}\right| \mathrm{d} S \mathrm{~d} t
\end{aligned}
$$

Nota-se que a quarta integral é calculada sobre a superfície do corpo $B$ e, consequentemente, influencia somente na condição de contorno de parede. As condições de contorno por ora relevantes serão discutidas a seguir:

\subsubsection{Condição de Parede}

A condição de parede consiste em impor nas equações governantes do escoamento a condição de aderência. Isso implica que as variações de velocidade são nulas:

$$
\begin{aligned}
\left.u^{i}\right|_{\text {parede }} & =\left(\beta_{j^{\prime}}^{i} u^{j^{\prime}}\right)_{\text {parede }}=0 \text { for }\left.u^{j^{\prime}}\right|_{\text {parede }}=\left.\delta u^{j^{\prime}}\right|_{\text {parede }}=0 \\
\left.\delta u^{i}\right|_{\text {parede }} & =\left(\beta_{j^{\prime}}^{i} \delta u^{j^{\prime}}+\delta \beta_{j^{\prime}}^{i} u^{j^{\prime}}\right)_{\text {parede }}=0 \\
\left.\delta u^{i}\right|_{\text {parede }} & =0
\end{aligned}
$$

A primeira integral na equação (3.49) é, então, calculada sobre a superfície do corpo $B$, mas sabendo-se da equação (3.50) que $\left.\delta u^{i}\right|_{\text {wall }}=0$, o primeiro termo dessa integral vai a zero, independente do termo interior aos colchetes. Os termos restantes dessa primeira integral são agrupados com a segunda integral (que também é calculada sobre a superfície do corpo e ao longo do tempo) obtendo, assim, a condição de parede:

$$
\int_{0}^{T} \oint_{B}\left[\left(\frac{\partial G}{\partial p}\left|\frac{\mathrm{d} S^{\prime}}{\mathrm{d} S}\right|-v \varphi_{i} n^{i}\right) \delta p+\left(\frac{\partial G}{\partial \mathcal{F}^{i}}\left|\frac{\mathrm{d} S^{\prime}}{\mathrm{d} S}\right|+v \varphi_{i}\right) \delta \mathcal{F}^{i}\right] \mathrm{d} S \mathrm{~d} t
$$

Como as variações $\delta p$ e $\delta \mathcal{F}^{i}$ são arbitrárias e independentes, a unica maneira de fazê-las ir a zero é impor as seguintes condições na solução adjunta, nas fronteiras sólidas:

$$
\left\{\begin{aligned}
\left.\varphi^{i} n_{i}\right|_{p} & =\left(\rho \frac{\partial G}{\partial p}\left|\frac{\mathrm{d} S^{\prime}}{\mathrm{d} S}\right|\right)_{\text {parede }} \\
-\left.\varphi_{i}\right|_{p} & =\left(\rho \frac{\partial G}{\partial \mathcal{F}^{i}}\left|\frac{\mathrm{d} S^{\prime}}{\mathrm{d} S}\right|\right)_{\text {parede }}
\end{aligned}\right.
$$


Essas relações impõe uma restrição na medida de mérito (3.24). Para que haja consistência na equação acima, a função $G$ deve satisfazer a seguinte identidade:

$$
\left.\frac{\partial G}{\partial p}\right|_{\text {parede }}=-\left.n^{i} \frac{\partial G}{\partial \mathcal{F}^{i}}\right|_{\text {parede }}
$$

Como resultado, qualquer uma das duas equações em (3.52) é uma condição de contorno adjunta adequada para o problema, acompanhada da identidade em (3.53). Além disso, todas as condições apresentadas em (3.52) são do tipo Dirichlet e restringem o mesmo número de variáveis $\varphi^{k}$ quanto a condição de não deslizamento restringe $u^{k}$. A variável $\Theta$ na parde é determinada pela solução adjunta da mesma forma que pressão é resultado da solução do escoamento ali. Dessa forma, as condições de contorno adjuntas na parede devem ser completamente consistentes com a estruturação do problema.

Uma maneira de demonstrar como essa última condição pode ser restritiva, parte-se de um caso no qual a função $G$ é uma projeção da força que o fluido exerce sobre o corpo em uma dada direção e

$$
G(\mathbf{n} \cdot \boldsymbol{\sigma} \cdot \mathbf{e})=\mathcal{F}^{k} e_{k}-p n^{k} e_{k}
$$

Aplicando a equação (3.53) na equação acima:

$$
\left.\frac{\partial G}{\partial p}\right|_{\text {parede }}=-\left.n^{i} \frac{\partial G}{\partial \mathcal{F}^{i}}\right|_{\text {parede }}=-n^{k} e_{k}
$$

enquanto ambas as equações (3.52) levam à seguinte condição de parede:

$$
\left.\varphi_{k}\right|_{p}=-\left(\rho e_{k}\left|\frac{\mathrm{d} S^{\prime}}{\mathrm{d} S}\right|\right)_{\text {parede }}
$$

Esse exemplo mostra que a restrição representada na equação (3.53) impõe na função $G$ a mesma relação entre $p g^{i j}$ e $\tau^{i j}$ que é expressa também na forma completa do tensor das tensões $\sigma^{i j}$.

\subsubsection{Escoamento ao Longe: Entrada}

Para esta condição, assume-se que o vetor normal é orientado para fora do domínio, de forma que a seção de entrada corresponda a região de fronteira do escoamento ao longe na qual $\mathbf{u} \cdot \mathbf{n}<0$. O vetor de velocidades imposto é conhecido 
e não nulo. Dessa forma, tem-se a seguinte condição linearizada para a entrada:

$$
\begin{aligned}
\left.u^{j^{\prime}}\right|_{e n} & =\left(\beta_{i}^{j^{\prime}} u^{i}\right)_{e n}=\text { conhecido } \\
\left.\delta u^{j^{\prime}}\right|_{e n} & =\left(\beta_{i}^{j^{\prime}} \delta u^{i}+\delta \beta_{i}^{j^{\prime}} u^{i}\right)_{e n}=\left.0 \quad \mathrm{e} \quad \delta \beta_{i}^{j^{\prime}}\right|_{\infty} \rightarrow 0 \\
\left.\delta u^{i}\right|_{e n} & =0
\end{aligned}
$$

onde admitiram-se que todas as variações métricas na fronteira de entrada são desprezíveis, hipótese essa tida como válida uma vez que a fronteira de entrada deve estar localizada suficientemente longe do corpo. Embora pareça uma simplicação mais apropriada para escoamentos externos é importante ressaltar que também é válida para escoamentos internos onde pode haver alguma liberdade no posicionamento da fronteira de entrada. Os resultados exibidos no capítulo 5 comprovam isso visto que os testes de validação neste trabalho foram realizados com escoamentos internos.

A condição linearizada é praticamente similar ao caso anterior, porém, agora, somente a primeira integral da equação (3.49) que se aplica para a condição de entrada no escoamento ao longe. Impondo a equação (3.57) em (3.49):

$$
\int_{0 \partial \mathcal{D}}^{T} \oint v\left(\delta \mathcal{F}^{i} \varphi_{i}-\delta p n^{i} \varphi_{i}\right) \mathrm{d} S \mathrm{~d} t
$$

Novamente, as variações físicas $\delta \mathcal{F}^{i}$ e $\delta p$ são arbitrárias e independentes. Consequentemente a única maneira de fazer com que a integral seja nula é assumindo que:

$$
\left\{\begin{array}{c}
\varphi_{i}=0 \\
n^{i} \varphi_{i}=0
\end{array}\right.
$$

Isoladamente a segunda equação impõe somente que $\boldsymbol{\varphi} \perp \mathbf{n}$. Entretanto, a primeira equação de (3.59) garante que a primeira integral da equação (3.49) se anule identicamente, com o mesmo efeito em (3.45). Como resultado, condições do tipo Dirichlet homogêneas são impostas na variável $\varphi$ fronteira de entrada do escoamento ao longe.

$$
\left.\varphi\right|_{i n}=0
$$

Novamente as condições de entrada adjuntas são similares às impostas nas variáveis $\mathbf{u}$ e $\delta \mathbf{u}$ nas equações do escoamento. Além disso, $\Theta$ é livre nessa fronteira bem como $p$ é na solução do escoamento. O fato de tanto as equações do escoamento quanto as adjuntas apresentarem o mesmo comportamento matemático, a equação (3.60) deve ser consistente com a estrutura do problema. 


\subsubsection{Escoamento ao Longe: Saída}

Admitindo a mesma orientação do vetor normal que foi admitida para a condição de entrada, a seção de saída é a fronteira do escoamento ao longe na qual u.n $\geq 0$.

Como foi proposto por Barkley et al. (BARKLEY; BLACKBURN; SHERWIN, 2002), na fronteira de saída impõe-se um valor constante de pressão e gradientes de velocidade na direção normal nulos. Como trata-se de um escalar, a condição de pressão ( $p=$ constant) não acarreta nenhuma variação métrica e, portanto, requer apenas que a parcela física da variação seja nula $(\delta p=0)$. Entretanto a derivada da velocidade certamente acarreta variações métricas.

$$
\begin{aligned}
u^{i^{\prime}} \mid j^{j^{\prime}} n_{j^{\prime}} & =\left.\beta_{k}^{i^{\prime}} u^{k}\right|^{r^{\prime}} \delta_{r^{\prime}}^{j^{\prime}} n_{j^{\prime}}=0 \\
& =\left.\beta_{k}^{i^{\prime}} u^{k}\right|^{r^{\prime}} \beta_{r^{\prime}}^{p} \beta_{p}^{j^{\prime}} n_{j^{\prime}}=\left.\beta_{k}^{i^{\prime}} u^{k}\right|^{p} n^{p} \\
\left.u^{k}\right|^{p} n_{p} & =0 \\
\left.\delta u^{i^{\prime}}\right|^{j^{\prime}} n_{j^{\prime}} & =\left.\delta \beta_{k}^{i^{\prime}} u^{k}\right|^{p} n_{p}+\left.\beta_{k}^{i^{\prime}} \delta u^{k}\right|^{p} n_{p}=0 \\
\left.\delta u^{k}\right|^{p} n_{p} & =0
\end{aligned}
$$

Dessa forma, impõe-se o seguinte conjunto de restrições no problema linearizado:

$$
\begin{cases}\mu \delta u^{i} \mid{ }^{j} n_{j} & =0 \\ \delta p & =0\end{cases}
$$

A primeira equação em (3.62) implica que $\delta \mathcal{F}^{i}=\delta \tau^{i j} n_{j}=\left.\mu \delta u^{j}\right|^{i} n_{j}$. Além disso, a equação (3.61) foi multiplicada por $\mu$ em (3.62), para indicar que deve ser imposta somente para escoamentos viscosos. Essa condição na sua forma original (3.61), não atende ao critério de viscosidade limite - isto é, não recuperará a condição de saída de Euler $\delta p=0$, no limite em que $\mu \rightarrow 0$. Utilizando-se desses resultados para simplificar a integral de superfície correspondente em (3.49), chega-se a:

$$
\int_{0 \partial \mathcal{D}}^{T}\left[\Theta n_{i}-u^{j} \varphi_{i} n_{j}-\nu\left(\left.\varphi_{i}\right|_{j}+\left.\varphi_{j}\right|_{i}\right) n^{j}\right] \delta u^{i} \mathrm{~d} S \mathrm{~d} t+\left.\int_{0 \partial \mathcal{D}}^{T} \oint_{j} \nu n_{j} \delta u^{j}\right|^{i} \varphi_{i} \mathrm{~d} S \mathrm{~d} t
$$

Assumindo, a princípio, que variações da forma $\delta u^{i}$ e $\left.n_{j} \delta u^{j}\right|^{i}$ são completamente independentes, abre-se a possibilidade de simplesmente fazer com que os termos que multiplicam tais variações sejam nulos e, assim, eliminar as integrais de superfície. Porém, ao tomar tal caminho, chega-se em seis condições de contorno 
na saída, o que sobrespecifica as equações adjuntas (HIRSCH, 1994a).

Uma forma de tratar o problema é considerar a segunda integral em (3.63) separadamente e tentar encontrar uma relação entre $\delta u^{i}$ e $\left.n_{j} \delta u^{j}\right|^{i}$. Para isso, lança-se mão do Teorema de Gauss. A integral é calculada na superfície de um volume de controle com espessura infinitesimal, $V_{o}$, que é definido sobre a fronteira de saída.Aqui, a constante $\nu$ é suprimida na derivação:

$$
\begin{aligned}
&\left.\int_{0 \partial V_{o}} \oint_{j} \delta u^{j}\right|^{i} \varphi_{i} \mathrm{~d} S \mathrm{~d} t=\iint_{0 V_{o}}^{T}\left(\left.\delta u^{j}\right|^{i} \varphi_{i}\right){ }_{j} \mathrm{~d} \bar{\xi} \mathrm{d} t=\iint_{0 V_{o}}\left(\left.\left.\delta u^{j}\right|_{j}\right|^{i} \varphi_{i}+\left.\left.\delta u^{j}\right|^{i} \varphi_{i}\right|_{j}\right) \mathrm{d} \bar{\xi} \mathrm{d} t \\
&\left.\int_{0 \partial V_{o}}^{T} n_{j} \delta u^{j}\right|^{i} \varphi_{i} \mathrm{~d} S \mathrm{~d} t=\iint_{0 V_{o}}^{T}\left[\left.\left(\left.\delta u^{j}\right|_{j} \varphi_{i}\right)\right|^{i}-\left.\left.\delta u^{j}\right|_{j} \varphi_{i}\right|^{i}+\left.\left(\left.\varphi_{i}\right|_{j} \delta u^{j}\right)\right|^{i}-\left.\left.\varphi_{i}\right|^{i}\right|_{j} \delta u^{j}\right] \mathrm{d} \bar{\xi} \mathrm{d} t \\
&\left.\int_{0 \partial V_{o}}^{T} n_{j} \delta u^{j}\right|^{i} \varphi_{i} \mathrm{~d} S \mathrm{~d} t=\int_{0 \partial V_{o}}^{T}\left\{\left.n^{i} \varphi_{i}\right|_{j} \delta u^{j}-\varphi_{i} n^{i}\left[\delta\left(J \beta_{q^{\prime}}^{k}\right) u^{q^{\prime}}\right]_{, k}\right\} d S d t \\
&=\left.\int_{0 \partial V_{o}}^{T} n^{i} \varphi_{i}\right|_{j} \delta u^{j} \mathrm{~d} S \mathrm{~d} t \\
&\left.\int_{0 \partial V_{o}}^{T} \oint_{j} \delta u^{j}\right|^{i} \varphi_{i} \mathrm{~d} S \mathrm{~d} t=\left.\int_{0 \partial V_{o}}^{T} n^{i} \varphi_{i}\right|_{j} \delta u^{j} \mathrm{~d} S \mathrm{~d} t
\end{aligned}
$$

onde a ordem da segunda derivada covariante mudou, como discutido no apêndice A, e utilizou-se a propriedade solenoidal que o campo $\varphi_{i}$ apresenta. Considera-se, também, que nenhuma variação geométrica afeta a fronteira de saída. Aqui ressalta-se que, como em qualquer fonteira fixa, todas as variações métricas desaparecem suavemente nas vizinhanças da fronteira de saída (seção D.6). É importante ressaltar que a equação (3.64) é uma propriedade de $\left(\left.n_{j} \delta u^{j}\right|^{i} \varphi_{i}\right)$, a qual o termo $\left(\varphi_{i} \delta u^{i} \mid{ }^{j} n_{j}\right)$ não apresenta. Isso é facilmente demonstrado fazendo com que o termo passe pelo mesmo procedimento descrito.

Aparentemente $\delta u^{i}{ }^{j} n_{j}$ é a única parcela de $\delta \mathcal{F}^{i}$ que independe de $\delta u^{i}$. Afinal, quando se fixa $\delta u^{i}$ em uma determinada superfície, só se pode variá-la na direção normal àquela superfície. Ocorre que a componente normal da variação é exatamente o que (3.62) torna igual a zero. Substituindo a equação (3.64) para 
o termo correspondente em (3.63), chega-se a:

$$
\begin{aligned}
& \int_{0 \partial \mathcal{D}}^{T}\left\{\left[\Theta n_{i}-u^{j} \varphi_{i} n_{j}-\nu\left(\left.\varphi_{i}\right|_{j}+\left.\varphi_{j}\right|_{i}\right) n^{j}\right] \delta u^{i}+\left.\nu \varphi_{j}\right|_{i} \delta u^{i} n^{j}\right\} \mathrm{d} S \mathrm{~d} t= \\
= & \int_{0 \partial \mathcal{D}}^{T}\left[\Theta n_{i}-u^{j} \varphi_{i} n_{j}-\nu \varphi_{i} \mid{ }_{j} n^{j}\right] \delta u^{i} \mathrm{~d} S \mathrm{~d} t
\end{aligned}
$$

e para fazer com que a integral acima se anule para uma variação $\delta u^{i}$ arbitrária, devem ser impostas as seguintes condições para as variáveis adjuntas na fronteira de saída.

$$
\left.\Theta n_{i}\right|_{\text {out }}=u^{j} n_{j} \varphi_{i}+\left.\nu \varphi_{i}\right|_{j} n^{j}
$$

A equação (3.66) representa um conjunto de três condições de saída mistas. Dessa forma, são impostas menos restrições para as variáveis adjuntas do que as atribuídas para a solução do escoamento. Além disso, elas são diferentes das condições exibidas em (3.62), e não é possível estabelecer uma analogia entre os dois conjuntos de condições.

Todavia, existe uma solução possível para essa incompatibilidade entre os conjuntos de condições de contorno. Atribuindo-se um valor constante porém arbitrário a $\Theta$ na saída, da mesma maneira que a pressão no escoamento é imposta na forma de uma condição de Dirichlet para escoamentos incompressíveis. Assim, chega-se ao seguinte conjunto de condições de contorno:

$$
\left\{\begin{array}{l}
\Theta=0 \\
u^{j} n_{j} \varphi_{i}=-\left.\nu \varphi_{i}\right|_{j} n^{j}
\end{array}\right.
$$

que atende ao propósito dessa derivação.

\subsubsection{Planos de Simetria}

O princípio básico da condição de simetria é que a velocidade normal e a derivada normal da pressão no plano de simetria são identicamente nulas. Há duas abordagens diferentes quanto às condições impostas ao vetor velocidade. Neste trabalho, será utilizada a abordagem na qual apenas a derivada normal da velocidade tangencial seja nula no plano de simetria. A velocidade tangencial é obtida por meio do operador de projeção $P_{j}^{i}$. Com isso, o conjunto de condições 
de contorno de simetria é dado por:

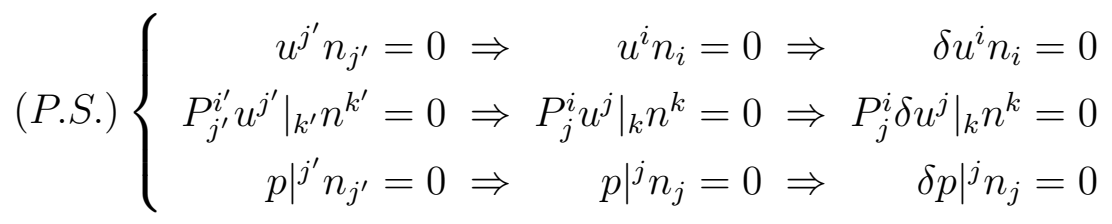

Substituindo $\delta \mathcal{F}^{i}$ pela sua definição e as duas primeiras equações de (3.68) pelos seus termos correspondentes em (3.49), chega-se a:

$$
\begin{aligned}
& \int_{0 \partial \mathcal{D}}^{T}\left[\nu \delta u^{i} \varphi_{i}\left|{ }_{j} n^{j}+\nu n^{j} \varphi_{j}\right|{ }_{i} \delta u^{i}+v \delta p n^{i} \varphi_{i}-\nu \varphi_{i} n^{i}\left(n_{k} \delta u^{k}\right)\left|{ }^{j} n_{j}-\nu n_{j} \delta u^{j}\right|^{i} \varphi_{i}\right] \mathrm{d} S \mathrm{~d} t \\
- & \int_{0 \partial \mathcal{D}}^{T}\left\{\nu \delta u^{k} P_{k}^{i} \varphi_{i} \mid{ }_{j} n^{j}+\varphi_{i} n^{i}\left[v \delta p-\nu\left(n_{k} \delta u^{k}\right) \mid{ }_{j} n^{j}\right]\right\} \mathrm{d} S \mathrm{~d} t
\end{aligned}
$$

onde utilizou-se a equação (3.64). A integral de superfície resultante leva às seguintes condições de contorno adjuntas:

$$
\left\{\begin{aligned}
n_{i} \varphi^{i} & =0 \\
\left.P_{k}^{i} \varphi_{i}\right|_{j} n^{j} & =0
\end{aligned}\right.
$$

as quais são análogas às do escoamento, exibidas na equação (3.68). Essas condições necessitam somente que $\boldsymbol{\varphi} \cdot \mathbf{n}$ e que a derivada normal da projeção tangencial de $\varphi$ no plano de simetria sejam iguais a zero. A equação (3.70) implica que:

$$
\left.\varphi_{k}\right|_{j} n^{j}=\left.n_{k}\left(n^{i} \varphi_{i}\right)\right|_{j} n^{j}
$$

que, por sua vez, leva ao adjunto do tensor das tensões, na forma:

$$
\nu\left(\left.\varphi_{i}\right|_{j}+\left.\varphi_{j}\right|_{i}\right) n^{j}=\nu\left[\left.n_{i}\left(n^{k} \varphi_{k}\right)\right|_{j} n^{j}+\left.\left(n^{j} \varphi_{j}\right)\right|_{i}\right]
$$

e integrando-se (3.72) sobre a superfície de um volume de controle com espessura infinitesimal sobre o plano de simetria, chega-se a:

$$
\left.\nu\left(\left.\varphi_{i}\right|_{j}+\left.\varphi_{j}\right|_{i}\right)\right|^{j}=\left.\left.\nu n_{i}\left(n^{k} \varphi_{k}\right)\right|_{j}\right|^{j}
$$

onde é importante ressaltar que o termo acima não é necessariamente nulo.

Uma projeção da segunda equação adjunta exibida em (3.48) na direção normal ao plano se simetria, combinada com as equações (3.68)-(3.73), leva a:

$$
\begin{aligned}
\partial_{o *}\left(n^{i} \varphi_{i}\right)-\left(n^{i} \varphi_{i}\right)\left|{ }_{j} u^{j}+\varphi_{j} u^{j}\right|{ }_{i} n^{i}+\Theta \mid{ }_{i} n^{i} & =\left.\left.\nu n^{i} \varphi_{i}\right|^{j}\right|_{j} \\
-\left[\left(n^{i} \varphi_{i}\right) \mid{ }_{k} n^{k}\right] n_{j} u^{j}+\varphi_{j} n^{j}\left(n_{k} u^{k}\right)\left|{ }_{i} n^{i}+\Theta\right|{ }_{i} n^{i} & =\left.\left.\nu\left(n^{k} \varphi_{k}\right)\right|_{j}\right|^{j} \\
\Theta \mid{ }_{i} n^{i} & =\left.\left.\nu\left(n^{k} \varphi_{k}\right)\right|_{j}\right|^{j}
\end{aligned}
$$


O resultado acima prova que a condição $\Theta \mid{ }_{i} n^{i}=0$, deve ser imposta para que não haja fluxo de quantidade de movimento através do plano de simetria.

Como resultado, a condição de derivada na direção normal da pressão adjunta deve ser imposta juntamente com as equações de (3.70). Dessa forma, as condições de contorno que devem ser impostas no plano de simetria são dadas por

$$
\left\{\begin{array}{r}
\left.\varphi^{i} n_{i}\right|_{s i m}=0 \\
\left.\left.P_{i}^{k} \varphi_{k}\right|_{j} n^{j}\right|_{s i m}=0 \\
\left.\left.\Theta\right|_{j} n^{j}\right|_{s i m}=0
\end{array}\right.
$$

que são análogas ao conjunto de condições de contorno do escoamento.

\subsection{Sensibilidades Não-Geométricas}

Nesta seção, será descrita uma metodologia para o cálculo de gradientes em relação a parâmetros não necessariamente relacionados à geometria do problema. Será utilizado o termo sensibilidades não geométricas, para quaisquer cálculos dessa natureza.

Como mencionado na seção 3.2, o problema adjunto foi modelado e derivado seguindo a metodologia de Jameson e seus colaboradores (NADARAJAH; JAMESON, 2007; JAMESON; MARTINELLI; PIERCE, 1998). Porém, na seção 3.1 mostrou-se a metodologia de Cacuci, que será utilizada agora. Ambas as metodologias apresentam muitas semelhaçnas porém se diferem em pontos específicos que serão brevemente explanados aqui.

A medida de mérito apresentada na equação (3.24) é a mesma utilizada por Cacuci, porém representada pelo funcional $I$ exibido na equação (3.1).

Aqui, nota-se que a equação (3.1) define o funcional $R$ como uma intergal sobre todo o domínio do escoamento $\mathcal{D}$ ao invés de uma integração sobre a superfície do corpo, como na equação (3.24). Na verdade, a equação (3.1) permite uma definição mais geral do problema. Para isso há uma infinidade de possibilidade para o trato das integrais de volume mas é possível recuperar as integrais de superfície por meio de distribuições. Reduz-se uma integração sobre o domínio $\mathcal{D}$ para uma sobre a superfície $B$, utilizando-se uma distribuição delta de Dirac:

$$
I=\frac{1}{T}\left\langle F[\mathbf{X}, \mathbf{a}, \rho], \delta_{(B)}\right\rangle
$$

onde $B$ representa uma superfície no espaço transformado, e o produto interno no domínio espaço-temporal é aquele exibido na equação (3.81) a seguir. Como 
está simplificado para uma integração sobre a superfície do corpo, o funcional $I$ pode receber o mesmo tratamento descrito na seção 3.2 .

Em ambas as metodologias, as equação de Navier-Stokes aparecem como restrição, uma vez que rege o fenômeno físico. As equações de Navier-Stokes estão expressas no conjunto (3.32), na forma de operadores. Na notação de Cacuci, corresponde à equação (3.6), que define o operador diferencial $\mathbf{N}$ e mostra que não há termo forçante $(\mathbf{Q}=0)$, posto que as EDPs são homogêneas:

$$
\begin{gathered}
\mathbf{N}[\mathbf{X}] \\
\left(\begin{array}{c|c}
\partial_{o}+(\mathbf{u} \cdot \nabla)-\nu \nabla^{2} & v \nabla \\
\hline \nabla \cdot & 0
\end{array}\right)\left(\begin{array}{c}
\mathbf{u} \\
\hline p
\end{array}\right)=\left(\begin{array}{c}
0 \\
\hline 0
\end{array}\right)
\end{gathered}
$$

Nota-se que as equações governantes não dependem explicitamente dos parâmetros de controle $\alpha$, quando formuladas diretamente no espaço físico.

O conjunto linearizado da equação (3.11) na notação de Cacuci é divido em duas partes: variações físicas expressas em (3.34) e variações geométricas expressas em (3.36):

$$
\begin{array}{r}
\mathbf{L} \delta \mathbf{X}-\mathbf{S} \delta \mathbf{a}=0 \\
\mathbf{N}_{X}^{\prime} \delta \mathbf{X}+\mathbf{N}_{\mathbf{a}}^{\prime} \delta \mathbf{a}=0
\end{array}
$$

A forma que o operador $\mathbf{B}[\mathbf{X}(\rho), \alpha]$ assume depende de cada caso. Porém, já fo visto que, embora haja dependência dos termos relativos à métrica e dos parâmetros de controle, a dependência desses últimos desaparece no caso linearizado. E isto vale para todas as condições exibidas na tabela 3.1,

$$
\begin{aligned}
\mathbf{B}[\mathbf{X}(\rho), \mathbf{a}] & =0 \\
\mathbf{B}_{X}^{\prime} \delta \mathbf{X} & =0
\end{aligned}
$$

A única exceção pode ser a fronteira de entrada, se essa não estiver suficientemente longe do corpo.

Como ambas as metodologias utilizam os mesmos operadores linearizados (L), a consequência disso é que tais operadores são efetivamente os mesmos. Em termos da equação (3.15), tem-se que:

$$
\langle\Phi, \mathbf{L} \delta \mathbf{X}\rangle=\left\langle\mathbf{L}^{*} \Phi, \delta \mathbf{X}\right\rangle-P[\Phi, \delta \mathbf{X}]_{s}
$$

onde $\Phi$ representa os multiplicadores de Lagrange. Este aspecto é a semelhança mais importante entre as metodologias. É conveniente associar o produto interno 
exibido na equação (3.8) com integrais de volume sobre o domínio $\Omega=\mathcal{D} \times(0, T)$ :

$$
\langle\mathbf{F}, \mathbf{G}\rangle=\int_{\Omega} \mathbf{F} \cdot \mathbf{G} \mathrm{d} \Omega=\int_{0}^{T} \int_{\mathcal{D}} \mathbf{F} \cdot \mathbf{G} J \mathrm{~d} \bar{\xi} \mathrm{d} t
$$

É também conveniente definir um outro produto interno sobre a superfície, como na equação (3.9) na qual o concomitante linear $P[\Phi, \delta \mathbf{X}]_{s}$ é calculado:

$$
\langle\mathbf{F}, \mathbf{G}\rangle_{S}=\int_{0}^{T} \int_{S} \mathbf{F} \cdot \mathbf{G} \mathrm{d} S \mathrm{~d} t
$$

Em ambos os casos o produto tensorial $(\mathbf{F} \cdot \mathbf{G})$ são calculados no espaço de estados enquanto as integrais são calculadas no espaço físico.

\subsubsection{Metodologia de Cacuci}

A principal diferença entre as metodologias de Jameson e Cacuci consiste na definição do funcional aumentado. Na abordagem proposta por Cacuci, as condições de contorno são impostas como restrições no problema variacional. Baseando-se na equação (3.10), chega-se a:

$$
\mathcal{J}(\mathbf{X}, \mathbf{a}, \Phi, \beta, \alpha)=I[\mathbf{X}, \mathbf{a}]-\langle\Phi, \mathbf{N}-\mathbf{Q}\rangle-\langle\beta, \mathbf{B}\rangle_{s}-\left\langle\alpha, \mathbf{a}-\mathbf{a}_{o}\right\rangle
$$

Como mencionado anteriormente, somente o operador das condições de contorno apresenta dependência explícita dos parâmetros de controle:

$$
\begin{aligned}
\mathbf{B}[\mathbf{X}(\rho), \mathbf{a}] & =0 \\
\mathbf{B}_{X}^{\prime} \delta \mathbf{X}+\mathbf{B}_{\mathbf{a}}^{\prime} \delta \mathbf{a} & =0
\end{aligned}
$$

onde $\mathbf{L} \equiv \mathbf{N}_{X}^{\prime}$ e $\mathbf{S} \equiv \mathbf{Q}_{\mathbf{a}}^{\prime}-\mathbf{N}_{\mathbf{a}}^{\prime}$. A variação toal de $G$ é dada pela equação (3.16):

$$
\begin{aligned}
\delta \mathcal{J}= & -\langle\delta \Phi, \mathbf{N}-\mathbf{Q}\rangle-\langle\delta \beta, \mathbf{B}\rangle_{s}-\left\langle\delta \alpha, \mathbf{a}-\mathbf{a}_{o}\right\rangle+\left\langle\mathbf{L}^{*} \Phi-F_{X}^{\prime}, \delta \mathbf{X}\right\rangle+ \\
& -\left\langle\beta, \mathbf{B}_{X}^{\prime} \delta X\right\rangle_{s}-\left[\left\langle P_{1}(\Phi), \mathbf{B}_{X}^{\prime} \delta \mathbf{X}\right\rangle_{s}+\left\langle\mathbf{B}^{*}(\Phi), \mathbf{A} \delta \mathbf{X}\right\rangle_{s}\right]+\left\langle\mathbf{F}_{\mathbf{a}}^{\prime}, \delta \mathbf{a}\right\rangle+ \\
& +\langle\Phi, \mathbf{S} \delta \mathbf{a}\rangle-\langle\alpha, \delta \mathbf{a}\rangle-\left\langle\beta, \mathbf{B}_{\mathbf{a}}^{\prime} \delta \mathbf{a}\right\rangle_{s}
\end{aligned}
$$

onde o operador $P[\Phi, \delta \mathbf{X}]_{s}$ foi decomposto de uma maneira não singular, porém assegurando que $\mathbf{A}$ é linearmente independente de $\mathbf{B}_{X}^{\prime}$.

Sempre que, as condições linearizadas não dependem explicitamente dos parâmetros de controle, como na equação (3.79), isso implica em $\mathbf{B}_{\mathbf{a}}^{\prime} \delta \mathbf{a}=0$. Consequentemente os quinto e último termo do lado direito da equação (3.85) são 
identicamente nulos:

$$
\left\langle\beta, \mathbf{B}_{X}^{\prime} \delta X\right\rangle_{s}=\left\langle\beta, \mathbf{B}_{\mathbf{a}}^{\prime} \delta \mathbf{a}\right\rangle_{s}=0
$$

Desse modo, o valor de $\beta$ é irrelevante e a abordagem proposta por Cacuci se mostra similar à proposta por Jameson. Assim sendo, o problema de contorno adjunto pode ser desenvolvido da mesma maneira proposta na seção 3.2. Porém a derivação ainda envolve uma decomposição do concomitante bilinear $P[\Phi, \delta \mathbf{X}]_{s}$ para obter o operador $\mathbf{B}^{*}(\Phi)$.

Um tipo de situação diferente ocorre quando as condições de contorno linearizadas dependem explicitamente dos parâmetros de controle. Quando isso ococrre, deve-se seguir a metodologia de Cacuci. Ou seja, quando $\mathbf{B}_{\mathbf{a}}^{\prime} \delta \mathbf{a} \neq 0$, a equação (3.84) deve ser considerada na sua forma completa, e a condição:

$$
\beta=-P_{1}(\Phi)
$$

passa a ser o ponto central do problema: não apenas é uma condição para $\delta G=0$ e para encontrar $\beta$ em termos de $\Phi$, mas é também parte do gradiente de sensibilidade. O gradiente de sensibilidade simplificado expresso na equação (3.21), para um extremo de $G$,

$$
\langle\alpha, \delta \mathbf{a}\rangle=\left\langle\mathbf{F}_{\mathbf{a}}^{\prime}, \delta \mathbf{a}\right\rangle+\langle\Phi, \mathbf{S} \delta \mathbf{a}\rangle-\left\langle\beta, \mathbf{B}_{\mathbf{a}}^{\prime} \delta \mathbf{a}\right\rangle_{s}
$$

onde $\beta$ é a variável que introduz a sensibilidade às condições de contorno em relação aos parâmetros de controle.

\subsubsection{Reformulação das Condições de Contorno}

Como já visto, ambas as metodologias, Jameson e Cacuci, apresentam semelhanças e modelam corretamente o problema adjunto. O único ponto da formulação que ainda necessita de tratamento é a decomposição do concomitante bilinear:

$$
P[\Phi, \delta \mathbf{X}]_{s}=\left\langle P_{1}(\Phi), \mathbf{B}_{X}^{\prime} \delta \mathbf{X}\right\rangle_{s}+\left\langle\mathbf{B}^{*}(\Phi), \mathbf{A} \delta \mathbf{X}\right\rangle_{s}
$$

e a questão crucial é como essa decomposição levará a um operador A que é linearmente independente de $\mathbf{B}_{X}^{\prime}$.

Para isso, cada condição de contorno será analisada nova e separadamente. Para os casos nos quais não há dependência dos parâmetros de controle, os operadores $\mathbf{A}$ e $P_{1}(\Phi)$ serão derivadas com base nas formas conhecidas $\mathbf{B}_{X}^{\prime}$ e $\mathbf{B}^{*}(\Phi)$ e, então checa-se se $\mathbf{A}$ e $\mathbf{B}_{X}^{\prime}$ são linearmente independentes. Na condição de 
entrada, onde $\mathbf{B}_{\mathbf{a}}^{\prime} \delta \mathbf{a} \neq 0$, a metodologia de Cacuci será seguida completamente.

A parte do concomitante bilinear $P[\Phi, \delta \mathbf{X}]_{s}$ relacionada às condições de contorno foi identificada em (3.49), e é reproduzida aqui em termo de produtos internos:

$$
\begin{aligned}
P[\Phi, \delta \mathbf{X}]_{s}= & -\left\langle\left(u^{j} \varphi_{i} n_{j}-\Theta n_{i}+\mathcal{H}_{i}\right) \delta u^{i}+v\left(\delta p n^{i}-\delta \mathcal{F}^{i}\right) \varphi_{i}\right\rangle_{s}+ \\
& +\left\langle\left(\frac{\partial G}{\partial p} \delta p+\frac{\partial G}{\partial \mathcal{F}^{k}} \delta \mathcal{F}^{k}\right)\left|\frac{\mathrm{d} S^{\prime}}{\mathrm{d} S}\right|\right\rangle_{B}
\end{aligned}
$$

O segundo termo no lado direito da integral é calculada sobre a superfície do corpo $B$ e, consequentemente, afeta só e diretamente a condição de contorno de parede. Além disso, os símbolos $\delta \mathcal{F}^{i}$ e $\mathcal{H}_{i}$ foram introduzidos para representarem os vetores:

$$
\begin{aligned}
\delta \mathcal{F}^{i} & \equiv \mu\left(\left.\delta u^{i}\right|^{j}+\left.\delta u^{j}\right|^{i}\right) n_{j} \\
\mathcal{H}_{i} & \equiv \nu\left(\left.\varphi_{i}\right|_{j}+\left.\varphi_{j}\right|_{i}\right) n^{j}
\end{aligned}
$$

\subsubsection{Condição de Parede}

Na condição de parede, todos os termos da equação (3.90) são relevantes. A primeira simplificação possível de ser feita é impor $\mathbf{u} \cdot \mathbf{n}=0$ na parede, já que o escoamento base atende às condições de contorno.

$$
\begin{aligned}
P[\Phi, \delta \mathbf{X}]_{B} & =\left\langle\left(\Theta n_{i}-\mathcal{H}_{i}\right) \delta u^{i}\right\rangle_{B}+\left\langle\left(\frac{\partial G}{\partial p}\left|\frac{\mathrm{d} S^{\prime}}{\mathrm{d} S}\right|-v \varphi_{i} n^{i}\right) \delta p\right\rangle_{B}+ \\
& +\left\langle\left(\frac{\partial G}{\partial \mathcal{F}^{i}}\left|\frac{\mathrm{d} S^{\prime}}{\mathrm{d} S}\right|+v \varphi_{i}\right) \delta \mathcal{F}^{i}\right\rangle_{B}
\end{aligned}
$$

De acordo com as equações (3.50) e (3.52), as condições de contorno podem ser escritas na forma de operadores como:

$$
\begin{array}{ll}
\mathbf{B}_{X}^{\prime} \delta \mathbf{X}=0 & \Rightarrow\left(\delta_{k}^{i} u^{k}+0 p\right)_{B}=0 \\
\mathbf{B}^{*}(\Phi) & \Rightarrow\left(\delta_{i}^{k} \varphi_{k}+0 \Theta\right)_{B}
\end{array}
$$

onde a equação para $\mathbf{B}^{*}(\Phi)$ deve considerar que a condição de parede adjunta não é homogênea.

Os termos $\mathbf{A} \delta \mathbf{X}$ e $P_{1}(\Phi)$ são dados por:

$$
\begin{aligned}
& \mathbf{A} \delta \mathbf{X}=v\left(\delta \mathcal{F}^{i}-\delta p n^{i}\right) \Leftrightarrow v\left[\mu\left(\left.\delta_{k}^{i}\right|^{j}+\left.\delta_{k}^{j}\right|^{i}\right) n_{j},-n^{i}\right] \cdot\left(\delta u^{k}, \delta p\right) \\
& P_{1}(\Phi)=-\left(\mathcal{H}_{i}-\Theta n_{i}\right) \Leftrightarrow-\left[\nu\left(\left.\delta_{i}^{k}\right|_{j}+\left.\delta_{j}^{k}\right|_{i}\right) n^{j},-n_{i}\right] \cdot\left(\varphi_{k}, \Theta\right)
\end{aligned}
$$

onde $\mathbf{A}$ e $P_{1}$ são operadores diferenciais. Com essas definições, os produtos in- 
ternos irão resgatar as seguintes integrais de superfície:

$$
\begin{aligned}
\left\langle P_{1}(\Phi), \mathbf{B}_{X}^{\prime} \delta \mathbf{X}\right\rangle_{B} & =\left\langle\left(\Theta n_{i}-\mathcal{H}_{i}\right) \delta u^{i}\right\rangle_{B} \\
\left\langle\mathbf{B}^{*}(\Phi), \mathbf{A} \delta \mathbf{X}\right\rangle_{B} & =\left\langle\varphi_{i} v\left(\delta \mathcal{F}^{i}-\delta p n^{i}\right)\right\rangle_{B}
\end{aligned}
$$

A equação (3.95) acima, corresponde ao primeiro termo do lado direito da equação (3.92). Entretanto, a equação (3.96) ainda deve ser combinada com o último termo do lado direito da equação (3.90) para obter as duas últimas integrais do lado direito da equação (3.92).

$$
\begin{gathered}
\left\langle\varphi_{i} v\left(\delta \mathcal{F}^{i}-\delta p n^{i}\right)\right\rangle_{B}+\left\langle\left(\frac{\partial G}{\partial p} \delta p+\frac{\partial G}{\partial \mathcal{F}^{k}} \delta \mathcal{F}^{k}\right)\left|\frac{\mathrm{d} S^{\prime}}{\mathrm{d} S}\right|\right\rangle_{B}= \\
=\left\langle\left(\frac{\partial G}{\partial p}\left|\frac{\mathrm{d} S^{\prime}}{\mathrm{d} S}\right|-v \varphi_{i} n^{i}\right) \delta p\right\rangle_{B}+\left\langle\left(\frac{\partial G}{\partial \mathcal{F}^{i}}\left|\frac{\mathrm{d} S^{\prime}}{\mathrm{d} S}\right|+v \varphi_{i}\right) \delta \mathcal{F}^{i}\right\rangle_{B}
\end{gathered}
$$

as quais, por sua vez, resgatam as condições de contorno de parede, exibidas nas equações (3.51) e (3.52).

Comparando-se as equações (3.93) e (3.94) vê-se que $\mathbf{B}_{X}^{\prime} \delta \mathbf{X}$ e $\mathbf{A} \delta \mathbf{X}$ são linearmente independentes.

\subsubsection{Escoamento ao Longe: Entrada}

Admitindo que o perfil de velocidades na entrada é conhecido, com um conjunto finito de parâmetros de controle:

$$
\begin{aligned}
u^{i} & =f^{i}\left(x, y, z, t ; \mathbf{a}^{k}\right) \\
\delta u^{i} & =\frac{\partial f^{i}}{\partial \mathbf{a}^{k}} \delta \alpha^{k}
\end{aligned}
$$

onde as velocidades podem ser espaço e tempo dependentes. Baseando-se na equação (3.84), o operador linearizado pode ser escrito como:

$$
\begin{aligned}
\mathbf{B}_{X}^{\prime} \delta \mathbf{X} & =\delta_{j}^{i} \delta u^{j}+0 \delta p \\
\mathbf{B}_{\mathbf{a}}^{\prime} \delta \mathbf{a} & =-\frac{\partial f^{i}}{\partial \mathbf{a}^{k}} \delta \mathbf{a}^{k}
\end{aligned}
$$

O fato do termo $\delta p$ estar multiplicado por zero significa que o mesmo não é restrito na fronteira de entrada.

O concomitante bilinear $P[\Phi, \delta \mathbf{X}]_{s}$, da equação (3.90) é dado por:

$$
P[\Phi, \delta \mathbf{X}]_{s}=-\left\langle\left(u^{j} \varphi_{i} n_{j}-\Theta n_{i}+\mathcal{H}_{i}\right) \delta u^{i}+v\left(\delta p n^{i}-\delta \mathcal{F}^{i}\right) \varphi_{i}\right\rangle_{s}
$$


Baseado na equação acima, os operadores $P_{1}$ e A são definidos por:

$$
\begin{aligned}
P_{1}(\Phi) & =-\left(\mathcal{H}_{i}-\Theta n_{i}\right)-u^{j} n_{j} \varphi_{i} \\
\mathbf{A} \delta \mathbf{X} & =v\left(\delta \mathcal{F}^{i}-\delta p n^{i}\right) \\
& =v\left[\mu\left(\left.\delta u^{i}\right|^{j}+\left.\delta u^{j}\right|^{i}\right)-\delta p g^{i j}\right] n_{j} \\
& =v \delta \sigma^{i j} n_{j}
\end{aligned}
$$

É possível notar que $\mathbf{A}$ e $\mathbf{B}_{X}^{\prime}$ são linearmente independentes. Substituindo os resultados obtidos acima nos seus respectivos termos na equação (3.89), obtem-se o seguinte operador adjunto de condição de contorno:

$$
\mathbf{B}^{*}(\Phi)\left(\varphi_{i}, \Theta\right)=\delta_{j}^{i} \varphi_{i}+0 \Theta=0
$$

que significa que $\Theta$ é livre, enquanto $\varphi_{i}=0$ na fronteira de entrada. É importante lembrar que a condição de contorno acima é precisamente a mesma da equação (3.60), para variações geométricas. Da equação (3.87) e utilizando a equação (3.104), chega-se a:

$$
\begin{aligned}
\beta_{i}=-P_{1}(\Phi) & =\left(\mathcal{H}_{i}-\Theta n_{i}\right)+u^{j} n_{j} \varphi_{i} \\
\beta_{i} & =\left(\mathcal{H}_{i}-\Theta n_{i}\right)
\end{aligned}
$$

Por fim, baseando-se na equação (3.88), como não há outras variações, o gradiente de sensibilidade fica dado por:

$$
\langle\alpha, \delta \mathbf{a}\rangle=-\left\langle\beta, \mathbf{B}_{\mathbf{a}}^{\prime} \delta \mathbf{a}\right\rangle_{s}=\left\langle\left(-\mathcal{H}_{i}+\Theta n_{i}\right) \frac{\partial f^{i}}{\partial \mathbf{a}^{k}} \delta \mathbf{a}^{k}\right\rangle_{s}
$$

Aqui a integral de superfície é calculada ao longo da fronteira de entrada.

\subsubsection{Escoamento ao Longe: Saída}

As condições de contorno dadas pelas equações (3.62) e (3.66) podem ser escritas na forma de vetor, como:

$$
\begin{aligned}
& \mathbf{B}_{X}^{\prime} \delta \mathbf{X}=\left(\left.\nu \delta u^{i}\right|^{j} n_{j}, v \delta p\right)=0 \\
& \mathbf{B}^{*}(\Phi)=\left(\Theta n_{i}-u^{j} n_{j} \varphi_{i}-\left.\nu \varphi_{i}\right|_{j} n^{j}\right)=0
\end{aligned}
$$


Somente a primeira integral da equação (3.90) representa o concomitante bilinear na fronteira de saída e, reagrupando seus termos, chega-se a:

$$
\begin{aligned}
P[\Phi, \delta \mathbf{X}]_{s} & =\left\langle v\left(\delta \mathcal{F}^{i}-\delta p n^{i}\right) \varphi_{i}\right\rangle_{s}+\left\langle\left[\Theta n_{i}-u^{j} n_{j} \varphi_{i}-\nu\left(\left.\varphi_{i}\right|_{j}+\left.\varphi_{j}\right|_{i}\right) n^{j}\right] \delta u^{i}\right\rangle_{s} \\
& =\left\langle\left(\left.\nu \delta u^{i}\right|^{j} n_{j}-v \delta p n^{i}\right) \varphi_{i}\right\rangle_{s}+\left\langle\left(\Theta n_{i}-u^{j} n_{j} \varphi_{i}-\left.\nu \varphi_{i}\right|_{j} n^{j}\right) \delta u^{i}\right\rangle_{s}
\end{aligned}
$$

O termo $\delta \mathcal{F}^{i}$ foi dividido entre produtos internos e a equação (3.64) foi utilizada para simplificar o segundo assim como foi feito na equação (3.65). Das expressões acima e das definições de $\mathbf{B}_{X}^{\prime} \delta \mathbf{X}$ e $\mathbf{B}^{*}(\Phi)$, é possíve derivar $\mathbf{A} \delta \mathbf{X}$ e $P_{1}(\Phi)$, dados por:

$$
\begin{aligned}
& \mathbf{A} \delta \mathbf{X}=\delta_{k}^{i} \delta u^{k} \\
& P_{1}(\Phi)=\left(\delta_{i}^{k},-n^{k}\right) \varphi_{k}
\end{aligned}
$$

Verificando, é possível notar que, dadas as fórmulas acima, $\mathbf{B}_{X}^{\prime} \delta \mathbf{X}$ e $\mathbf{A} \delta \mathbf{X}$ são linearmente independentes.

\subsubsection{Planos de Simetria}

O conjunto de condições de contorno para planos de simetria é formado pelas equações (3.68) e (3.75) para os problemas físico e adjunto respectivamente. Na forma de operadores, essas condições são expressas da seguinte maneira:

$$
\begin{aligned}
& \mathbf{B}_{X}^{\prime} \delta \mathbf{X}=\left(\delta u^{i} n_{i}, P_{j}^{i} \delta u^{j}\left|{ }_{k} n^{k}, \delta p\right|^{j} n_{j}\right)=0 \\
& \mathbf{B}^{*}(\Phi)=\left(\varphi^{i} n_{i},\left.P_{k}^{i} \varphi_{i}\right|_{j} n^{j}, \Theta \mid{ }_{j} n^{j}\right)=0
\end{aligned}
$$

O concomitante bilinear é, mais uma vez, dado pela primeira integral do lado direito da equação (3.90) sem o seu primeiro termo já que $\mathbf{u} \cdot \mathbf{n}=0$. Entretanto, é conveniente substituir $\delta \mathcal{F}^{i}$ e $\mathcal{H}_{i}$ por suas respectivas definições e utilizar a equação (3.64):

$$
\left\langle\left. n_{j} \delta u^{j}\right|^{i} \varphi_{i}\right\rangle_{s}=\left\langle\left. n^{i} \varphi_{i}\right|_{j} \delta u^{j}\right\rangle_{s}
$$

Chegando a:

$$
\begin{aligned}
P[\Phi, \delta \mathbf{X}]_{s} & =\left\langle\Theta n_{i} \delta u^{i}-v \delta p n^{i} \varphi_{i}+\nu\left(\varphi_{i} \delta u^{i}\left|{ }_{j} n^{j}-\delta u^{i} \varphi_{i}\right|_{j} n^{j}\right)\right\rangle_{s} \\
& =\left\langle\Theta n_{i} \delta u^{i}+\nu \varphi_{i} P_{k}^{i} \delta u^{k}\left|{ }_{j} n^{j}-\nu \delta u^{i} n_{i}\left(n^{k} \varphi_{k}\right)\right|_{j} n^{j}\right\rangle_{s}+ \\
& -\left\langle v \delta p n^{i} \varphi_{i}-\nu \varphi_{i} n^{i}\left(n_{k} \delta u^{k}\right)\left|{ }_{j} n^{j}+\nu \delta u^{i} P_{i}^{k} \varphi_{k}\right|{ }_{j} n^{j}\right\rangle_{s}
\end{aligned}
$$

Nota-se que os operadores $\left.\delta p\right|^{j} n_{j}$ e $\Theta \mid{ }_{j} n^{j}$ não aparecem acima e, além idsso, dois termos parecem estar fora do lugar. Esses termos serão considerados separada- 
mente:

$$
\begin{aligned}
\Delta & \equiv \nu\left\langle\left[\left.\varphi_{i} n^{i}\left(n_{k} \delta u^{k}\right)\right|_{j}-\left.\delta u^{i} n_{i}\left(n^{k} \varphi_{k}\right)\right|_{j}\right] n^{j}\right\rangle_{s} \\
& =\nu\left\langle\left.\left[\left.\varphi_{i} n^{i}\left(n_{k} \delta u^{k}\right)\right|_{j}-\left.\delta u^{i} n_{i}\left(n^{k} \varphi_{k}\right)\right|_{j}\right]\right|^{j}\right\rangle \\
& =\nu\left\langle\left.\left.\left(n^{i} \varphi_{i}\right)\right|^{j}\left(n_{k} \delta u^{k}\right)\right|_{j}+\left.\left.\varphi_{i} n^{i}\left(n_{k} \delta u^{k}\right)\right|_{j}\right|^{j}\right\rangle+ \\
& -\nu\left\langle\left.\left.\left(n^{k} \varphi_{k}\right)\right|_{j}\left(\delta u^{i} n_{i}\right)\right|^{j}+\left.\left.\delta u^{i} n_{i}\left(n^{k} \varphi_{k}\right)\right|_{j}\right|^{j}\right\rangle \\
& =\left\langle\left.\varphi_{i} n^{i} \delta p\right|^{k} n_{k}-\left.\delta u^{i} n_{i} \Theta\right|_{k} n^{k}\right\rangle
\end{aligned}
$$

Os dois primeiros termos nas terceira e quarta linhas tiveram suas variâncias ajustadas por meio do tensor métrico. Com isso, elas simplesmente se cancelam entre si. Os termos restantes estão exibidos na eq. (3.113) representados por $\Delta$, sendo esses os termos faltantes. Baseando-se no resultado acima, a equação (3.112) é expressa em um formato diferente:

$$
\begin{aligned}
P[\Phi, \delta \mathbf{X}]_{s} & =\left\langle\left[\Theta-\nu\left(n^{k} \varphi_{k}\right) \mid{ }_{j} n^{j}\right] \delta u^{i} n_{i}+\left.\nu \varphi_{i} P_{k}^{i} \delta u^{k}\right|_{j} n^{j}\right\rangle_{s}+ \\
& -\left\langle\left[v \delta p-\nu\left(n_{k} \delta u^{k}\right) \mid{ }_{j} n^{j}\right] n^{i} \varphi_{i}+\left.\nu \delta u^{i} P_{i}^{k} \varphi_{k}\right|_{j} n^{j}\right\rangle_{s}
\end{aligned}
$$

Os operadores $\mathbf{A} \delta \mathbf{X}$ e $P_{1}(\Phi)$, são definidos como:

$$
\begin{aligned}
& P_{1}(\Phi)=\left(\left[\Theta-\nu\left(n^{k} \varphi_{k}\right) \mid{ }_{j} n^{j}\right], \nu \varphi_{i}, 1\right) \\
& \mathbf{A} \delta \mathbf{X}=-\left(\left[v \delta p-\left.\nu\left(n_{k} \delta u^{k}\right)\right|_{j} n^{j}\right], \nu \delta u^{i}, 1\right)
\end{aligned}
$$

Onde o terceiro componente dos vetores acima fazem surgir uma nova integral de superfície na equação (3.114):

$$
\left\langle\left.\delta p\right|^{j} n_{j}-\left.\Theta\right|_{j} n^{j}\right\rangle_{s}=0
$$

que corresponde a uma maneira de fugir da dificuldade com os termos faltantes na equação (3.113). De qualquer forma, é fácil notar que $\mathbf{B}_{X}^{\prime} \delta \mathbf{X}$ e $\mathbf{A} \delta \mathbf{X}$ são linearmente independentes.

\subsubsection{Considerações Sobre a Derivação}

O conjunto de equações adjuntas, exibido na equação (3.48), é válido para qualquer sistema de coordenadas, seja qual for a transformação realizada. O conjunto, em notação simbólica:

$$
\left\{\begin{aligned}
\partial_{o *} \boldsymbol{\varphi}-(\mathbf{u} \cdot \nabla) \boldsymbol{\varphi}+(\nabla \mathbf{u})^{T} \cdot \boldsymbol{\varphi}+\nabla \Theta-\nu \nabla^{2} \boldsymbol{\varphi} & =0 \\
\nabla \cdot \boldsymbol{\varphi} & =0
\end{aligned}\right.
$$


Para fins de comparação, exibe-se o conjunto de equações de Navier-Stokes, que regem o fenômeno físico:

$$
\left\{\begin{aligned}
\partial_{o} \mathbf{u}+(\mathbf{u} \cdot \nabla) \mathbf{u}+v \nabla p-\nu \nabla^{2} \mathbf{u} & =0 \\
\nabla \cdot \mathbf{u} & =0
\end{aligned}\right.
$$

Com essa comparação, é evidente que ambas as equações são análogas, com diferença, apenas, nos termos convectivos que apresentam sinais contrários. Entretanto, os mecanismos de evolução no tempo e difusivos são os mesmos.

Um resumo de todas as condições de contorno derivadas nos itens anteriores, está expresso na tabela 3.1. Para fins de comparação, as condições do contorno para o problema físico, estão exibidas lado a lado com as condições de contorno adjuntas.

Tabela 3.1: Condições de contorno para os problemas físico e adjunto. A variável $\varphi$ representa a norma do vetor de velocidades adjunto, $\varphi=\|\varphi\|$, e o sobrescrito ()$^{t}$ denota um vetor que é tangente ao plano de simetria.

\begin{tabular}{lll}
\hline Fronteira & Escoamento & Adjunto \\
\hline Tempo & $\left.\mathbf{u}\right|_{t=0}=\mathbf{u}_{o}$ & $\left.\boldsymbol{\varphi}\right|_{t^{*}=0}=0$ \\
Entrada & $u^{i}=f^{i}\left(\xi^{j}, t ; \alpha^{k}\right)$ & $\boldsymbol{\varphi}=0$ \\
Saída & $\frac{\partial \mathbf{u}}{\partial n}=0$ & $\frac{\partial \boldsymbol{\varphi}}{\partial n}=-\frac{(\mathbf{u} \cdot \mathbf{n}) \boldsymbol{\varphi}}{\nu}$ \\
& $p$ fixa & $\Theta=0$ \\
Parede & $\mathbf{u}=0$ & $\boldsymbol{\varphi}=-\rho \frac{\partial G}{\partial \mathcal{F}}\left|\frac{\mathrm{d} S^{\prime}}{\mathrm{d} S}\right|$ \\
Plano Simet. & $\mathbf{u} \cdot \mathbf{n}=0$ & $\boldsymbol{\varphi} \cdot \mathbf{n}=0$ \\
& $\frac{\partial \mathbf{u}^{t}}{\partial n}=\frac{\partial p}{\partial n}=0$ & $\frac{\partial \boldsymbol{\varphi}^{t}}{\partial n}=\frac{\partial \Theta}{\partial n}=0$ \\
\hline
\end{tabular}

A condição de contorno de parede impõe uma restrição no núcleo do funcional $G(\mathbf{n} \cdot \boldsymbol{\sigma} \cdot \mathbf{e})$,

$$
\left.\frac{\partial G}{\partial p}\right|_{\text {parede }}=-\left.\frac{\partial G}{\partial \mathcal{F}} \cdot \mathbf{n}\right|_{\text {parede }}
$$

Fazendo com que as variáveis adjuntas sejam solução das equações acima e substituindo-as na variação aumentada da equação (3.45), eliminam-se todas as variações físicas da mesma. Como resultado, a expressão de $\delta I$ (considerando 
apenas variações geométricas) se reduz a:

$$
\begin{aligned}
T \delta I & =\int_{0} \oint_{B} G \delta\left|\frac{d S^{\prime}}{d S}\right| \mathrm{d} S \mathrm{~d} t+\int_{\Omega}\left(\frac{\Theta}{J}-\varphi_{i} u^{i}\right)\left[\delta\left(J \beta_{q^{\prime}}^{k}\right) u^{q^{\prime}}\right]_{, k} \mathrm{~d} \Omega+ \\
& -\int_{\Omega} \psi_{r^{\prime}}\left\{\partial_{o}\left(\delta J u^{r^{\prime}}\right)+\left[\delta\left(J \beta_{n^{\prime}}^{j}\right)\left(u^{r^{\prime}} u^{n^{\prime}}+g^{r^{\prime} n^{\prime}} p v-\nu u_{, p^{\prime}}^{r^{\prime}} g^{p^{\prime} n^{\prime}}\right)\right]_{, j}\right\} \mathrm{d} \Omega
\end{aligned}
$$

Essa equação pode ser utilizada para calcular o gradiente de sensibilidade sem a necessidade de simulações computacionais adicionais para o escoamento base, como são necessárias em outros métodos para o cálculo de gradientes. O método adjunto oferece essa vantagem no quesito custo computacional. Independente de quantos sejam os parâmetros de controle, não serão necessárias novas simulações. Outro ponto no qual o método se mostra vantajoso é quanto à flexibilidade para se modificar a medida de mérito, permitindo, assim, um extenso conjunto de aplicações hidrodinâmicas possíveis.

Dadas todas as condições de contorno expressas segundo a abordagem de Cacuci, a expressão do gradiente de sensibilidade quando há apenas variações não-geométricas é dado por:

$$
\langle\alpha, \delta \mathbf{a}\rangle=\int_{0}^{T} \oint_{S_{i}}\left[-\nu\left(\left.\varphi_{i}\right|_{j}+\left.\varphi_{j}\right|_{i}\right) n^{j}+\Theta n_{i}\right] \frac{\partial f^{i}}{\partial \mathbf{a}^{k}} \delta \mathbf{a}^{k} \mathrm{~d} S \mathrm{~d} t
$$

Como já mencionado, a metodologia adotada por Cacuci difere da adotada por Jameson no cálculo do funcional aumentado e Cacuci ainda impões restrições nas condições de contorno. Porém a linha seguida por Cacuci permite o cálculo de sensibilidades não geométricas de maneira bastante eficiente. Dessa forma, foram obtidas duas equações para parcelas distintas do gradiente: a eq. (5.25) referente à variações de geometria e a eq. (3.121) referente à variações não geométricas. É importante frisar que as metodologias não são excludentes. Naquela proposta por Cacuci é possível calcular sensibilidades além das geométricas. Se forem consideradas apenas variações geométricas a metodologia proposta por Cacuci pode ser simplificada e resultar na de Jameson. Assim, nos resultados exibidos nos próximos capítulos, será utilizada a Metodologia de Cacuci para o cálculo de sensibilidades não geométricas e a de Jameson para o cálculo de sensibilidades geométricas.

Com as fórmulas para os gradientes definidas é possível resolver as equações do escoamento e adjuntas para, em seguida, calcular numericamente tais 
gradientes. Os códigos computacionais que serão utilizados são o SEMTEX e o FreeFem ++ , como já mencionado. No capítulo a seguir, capítulo 4, serão exibidas as metodologias do método número empregado em cada código. 


\section{SimulaÇÕeS NuMÉRICAS}

Neste capítulo serão apresentadas as metodologias que embasam os métodos numéricos empregados nas simulações computacionais que resolvem as equações do escoamento e adjuntas. Todas as simulações tanto das equações do escoamento quanto das adjuntas foram realizadas com dois códigos computacionais: SEMTEX e FreeFem ++ . O primeiro código foi desenvolvido por H.M. Blackburn. com o objetivo de estudar instabilidades hidrodinâmicas. Um dos autores de (BARKLEY; BLACKBURN; SHERWin, 2002), Prof. S. Sherwin do Imperial College, em conjunto com seu grupo, participou na elaboração do código SEMTEX. Um dos membros desse grupo, Prof. Xuerui Mao da Universidade de Nottingham (MAO, 2010), permitiu que esse código fosse utilizado neste trabalho e, por meio dos Profs. B. S. Carmo e J. R. Meneghini, foi obtido acesso a esse código. Já o FreeFem + + foi desenvolvido por F. Hecht (HECHT, 2012) em colaboração com outros pesquisadores para resolver sistemas de equações diferenciais parciais.

Ambos os códigos são capazes de resolver tanto as equações governantes do escoamento quanto as equações adjuntas. A diferença entre ambos está basicamente no método utilizado por cada um. O SEMTEX utiliza o método dos elementos espectrais. Este código se torna mais apropriado para os propósitos desse trabalho pois têm implementada a condição de contorno adjunta para a saída, de maneira similar à exibida na tabela (3.1). Aliás, em contato com um membro do grupo do Prof. S. Sherwin, Dr. X. Mao (MAO, 2010), soube-se que o SEMTEX tem implementadas todas as condições listadas na tabela, embora sua concepção visasse outras finalidades distintas daqueles deste trabalho. Já o FreeFem ++ utiliza o método de elementos finitos, baseado na forma fraca das equações a serem resolvidas. O FreeFem ++ permite a implementação das equações do escoamento e adjuntas bem como das respectivas condições de contorno na forma apresentada na tabela citada anteriormente.

A seguir serão apresentados o modelo matemático do escoamento e os métodos dos elementos espectrais e de elementos finitos. 


\subsection{Modelo Matemático do Escoamento e Equa- ções Adjuntas}

Neste trabalho todos os problemas de estudo serão escoamentos viscosos e incompressíveis regidos pelas equações de Navier-Stokes. Com essas duas características, as equações na sua forma dimensional são dadas por:

$$
\left\{\begin{aligned}
\partial_{o} \mathbf{u}+(\mathbf{u} \nabla) \mathbf{u}+v \nabla p-\nu \nabla^{2} \mathbf{u} & =0 \\
\nabla \mathbf{u} & =0
\end{aligned}\right.
$$

onde $\mathbf{u}=(u, v, w)$ é o vetor de velocidades, $p$ é o campo de pressão e $\nu$ é a viscosidade cinemática do fluido. O dado sistema de equações além de governar os escoamentos estudados, é utilizado como base para a derivação das equações adjuntas. Como neste trabalho o Método Adjunto será utilizado na sua forma contínua, as equações adjuntas são derivdas diretamente das equações constitutivas. As equações adjuntas foram exibidas já na seção (3.3.3), e são resgatadas abaixo;

$$
\left\{\begin{array}{r}
\partial_{o} * \boldsymbol{\varphi}-(\mathbf{u} \cdot \nabla) \boldsymbol{\varphi}+(\nabla \mathbf{u})^{T} \cdot \boldsymbol{\varphi}+\nabla \Theta-\nu \nabla^{2} \boldsymbol{\varphi}=0 \\
\nabla \cdot \boldsymbol{\varphi}=0
\end{array}\right.
$$

\subsection{Equações Adimensionais}

É possível encontrar a forma adimensional das equações (4.1) e (4.2). Para tal, faz-se uso das seguintes relações:

$$
\begin{aligned}
& \tilde{x}=\frac{x}{L_{R}} \quad \tilde{\mathbf{u}}=\frac{\mathbf{u}}{U_{R}} \quad \tilde{\boldsymbol{\varphi}}=U_{R}^{2} \boldsymbol{\varphi} \\
& \tilde{t}=\frac{U_{R} t}{L_{R}} \quad ; \quad \tilde{p}=\frac{p}{\rho U_{R}^{2}} \quad ; \quad \tilde{\Theta}=U_{R} \Theta \\
& \tilde{t}^{*}=\frac{U_{R} t^{*}}{L_{R}} \quad \tilde{\tau}=\frac{\tau}{\rho U_{R}^{2}} \quad \tilde{T}=\frac{U_{R} T}{L_{R}}
\end{aligned}
$$

onde $L_{R}$ e $U_{R}$ são as escalas de referência (fixas) do comprimento e da velocidade, respectivamente. É importante ressaltar que em escoamentos bidimensionais $\mathrm{d} S^{\prime}$ é um diferencial sobre a superfície que é definido como $\mathrm{d} \tilde{S}^{\prime}=\mathrm{d} S^{\prime} / D$ e, também define-se: $\mathrm{d} t / T=\mathrm{d} \tilde{t} / \tilde{T}$. Com essas definições é possível obter as equações de 
Navier-Stokes adimensionais:

$$
\left\{\begin{array}{r}
\tilde{\partial}_{o} \tilde{\mathbf{u}}+(\tilde{\mathbf{u}} \cdot \tilde{\nabla}) \tilde{\mathbf{u}}+\tilde{\nabla} \tilde{p}-\frac{1}{R e} \tilde{\nabla}^{2} \tilde{\mathbf{u}}=0 \\
\tilde{\nabla} \cdot \tilde{\mathbf{u}}=0
\end{array}\right.
$$

e, consequentemente, as equações adjuntas adimensionais:

$$
\left\{\begin{aligned}
\tilde{\partial}_{o} \tilde{\boldsymbol{\varphi}}-(\tilde{\mathbf{u}} \cdot \tilde{\nabla}) \tilde{\boldsymbol{\varphi}}+(\tilde{\nabla} \tilde{\mathbf{u}})^{T} \cdot \tilde{\boldsymbol{\varphi}}+\tilde{\nabla} \tilde{\Theta}-\frac{1}{R e} \tilde{\nabla}^{2} \tilde{\boldsymbol{\varphi}} & =0 \\
\tilde{\nabla} \cdot \tilde{\boldsymbol{\varphi}} & =0
\end{aligned}\right.
$$

\subsection{O Método dos Elementos Finitos}

Esta seção tem como objetivo descrever sucintamente os fundamentos do Método dos Elementos Finitos, sobre o qual está baseado o FreeFem ++ e que serve como bsae para o Método dos Elementos Espectrais/hp, sobre o qual o código SEMTEX está baseado. As formulações aqui exibidas foram extraídas e adaptadas do livro (KARNIADAKIS; SHERWIN, 2005) e das notas de aula do curso do Prof. Bruno (CARMo, 2014). Não é a intenção deste trabalho se aprofundar no método mas sim fazer uso dele de forma consciente. Desta forma, se o leitor desejar mais detalhes do mesmo, está convidado a consultar as referências citadas.

O Método dos Elementos Finitos (MEF) basicamente, um método que visa resolver sistemas de equações diferenciais parciais utilizando a discretização do conjunto universo (domínio) desse sistema de equações. Para isso, o domínio é discretizado em elementos e a solução da equação diferencial do problema é obtida a partir de aproximações locais em cada elemento. A obtenção dessas aproximações é feita a partir de um conjunto de funções (base de expansão) que é estabelecido para formar aproximações por meio de combinações lineares e restrições para assegurar a continuidade das aproximações entre elementos. O Método dos Elementos Espectrais (MEE) combina propriedades do Método de Elementos Finitos e dos Métodos Espectrais (ME). Do MEF são utilizadas as propriedades já citadas enquanto do ME, absorve-se a idéia de utilizar funções de alta ordem como funções base, garantindo a convergência exponencial característica dessa classe de métodos. É utilizada uma representação única de uma função $u(x)$ em todo o domínio do problema, truncando uma expansão em série (KARNIADAKIS; SHERWIN, 2005):

$$
u(x) \approx u_{N}(x)=\sum_{n=0}^{N} \hat{u_{n}} \phi_{n}
$$

sendo $\phi$ as funções de base. Dessa forma, a aproximação da função $u(x)$ é subs- 
tituida na equação do problema (seja ela integral ou diferencial) e, por meio de um método de minimização do resíduo proveniente do truncamento da série, os coeficientes ${ }_{n}$ são determinados. Para o MEE - SEMTEX -, são utilizados, normalmente, como funções base polinômios de Chebyshev $T_{n}(x)$ ou polinônios da família dos polinômios de Jacobi $P_{n}^{\alpha, \beta}$. Uma importante característica dessa família de polinômios é a ortogonalidade dos mesmos em relação à função $(1-x)^{\alpha}(1+x)^{\beta}$ no intervalo $[-1,1]$. Para o FreeFem ++ são utilizados polinômios de Lagrange.

O emprego do MEE é recomendado para problemas que necessitam de simulações por longos intervalos de tempo o que consequentemente acarreta na necessidade de longas integrações temporais. É também conhecido por Método $/ h p$ pois possibilita dois tipos de convergência a saber: $h$ e $p$. A primeira ocorre na redução do tamanho dos elementos da malha ( $h$ é a dimensão do elemento). Já a segunda ocorre com o aumento grau do polinômio interpolador da base.

Novamente, destaca-se que serão utilizados dois códigos neste trabalho para fins de comparação entre eles de modo a contornar eventuais dificuldades numéricas encontradas em cada um dos códigos. Isso ilustra, também, a independência do Método Adjunto na sua forma contínua em relação ao código computacional escolhido. As subseções seguintes (4.3.1) e (4.3.2) se aplicam tanto ao SEMTEX quanto ao FreeFem ++ .

\subsubsection{O Método dos Resíduos Ponderados}

Os Métodos de Resíduos Ponderados são métodos de aproximação utilizados para a solução de equações (ou sistemas de equações) diferenciais, parciais ou não. A idéia por trás do método consiste em aproximar a solução por uma série truncada conforme mostrado na eq. (4.6) de modo que ela satisfaça um conjunto finito de condições. Esse conjunto especificado definirá o método numérico empregado na solução do problema (CARMO, 2005). Dentre os disponíveis vale citar o Método Geral, Método de Colocação, Método de Newton, Método do Subdomínio, Método dos Mínimos Quadrados e Método de Galerkin.

Para explicar sucintamente a idéia do Método dos Resíduos Ponderados, considera-se uma equação diferencial linear em um domínio $\Omega$, sujeita a condições iniciais e de contorno apropriadas, dada por:

$$
\mathbb{L}(u)=0
$$


A solução aproximada tem a seguinte forma (KARNIADAKIS; SHERWIN, 2005):

$$
u(\vec{x}, t) \approx u^{\delta}(x, t)=u_{0}(\vec{x}, t)+\sum_{n=1}^{N_{g l}} \hat{u}_{n}(t) \Phi_{n}(\vec{x})
$$

onde $\Phi(x)$ são as funções de base e $\hat{u_{n}}$ são os coeficientes a serem determinados, $u_{0}(x, t)$ satisfaz as condições iniciais e de contorno e $N_{g l}$ é o número de graus de liberdade da aproximação. Substituindo a eq. (4.8) na eq. (4.7), que governa o problema, incutirá em um resíduo não nulo provieniente da aproximação feita, dado por:

$$
\mathbb{L}\left(u^{\delta}\right)=R\left(u^{\delta}\right)
$$

Para que haja uma única maneira de determinar os coeficientes ${ }_{n}$, impõe-se uma restrição ao resíduo de forma que a eq. (4.9) seja reduzida a um sistema de equações diferenciais ordinárias em $\hat{u}_{n}$. Se a eq. (4.7) for independente do tempo, os coeficientes serão determinados através da solução de um sistema de equações algébricas (CARMO, 2014). A restrição imposta ao resíduo é:

$$
<v_{i}(x), R>=0, i=1, \ldots, N_{g l}
$$

onde $v(x)$ é chamada função de teste e $<f, g>$ denota o produto interno dado por:

$$
<f, g>=\int_{\Omega} f(x) g(x) d \vec{x}
$$

A metodologia numérica de solução para o Método dos Resíduos Ponderados varia de acordo com a escolha da função teste. Tanto o MEE como o MEF utilizam o Método dos Resíduos Ponderados com formulação de Galerkin, portanto neste trabalho será explicada somente essa formulação, de maneira sucinta.

\subsubsection{Formulação de Galerkin}

$\mathrm{Na}$ formulação de Galerkin, as funções teste $v_{i}(x)$ são as mesmas funções base $\Phi_{i}(x)$ utilizadas para aproximar a solução no domínio do problema. Para construir a forma fraca de eq. (4.7) calcula-se o produto interno da mesma com a função:

$$
<v, \mathbb{L}(u)>=0
$$

Nessa formulação, as condições de contorno Dirichlet são especificadas de forma explícita. Já as condições de Neumann são especificadas naturalmente no problema, após integrar a eq. (4.12) por partes e assumindo que a função teste na fronteira é nula. Para incluir condições de contorno do tipo Dirichlet não homo- 
gêneas, é necessário que a base de expansão englobe funções que não se anulem na fronteira. Para isso, a aproximação da solução $u^{\delta}$ é decomposta em duas parcelas:

$$
u^{\delta}=u^{\mathbb{H}}+u^{\mathbb{D}}
$$

onde $u^{\mathbb{H}}$ é a parcela que se anula nas fronteiras onde há uma condição de contorno Dirichlet especificada e $u^{\mathbb{D}}$ é a parcela que satisfaz as condições de Dirichlet. Substituindo a eq.(4.13) na eq.(4.12) integrada por partes (Forma fraca do problema) chega-se a uma equação integral possível de ser resolvida.

A idéia do método, de maneira bem geral e resumida, consiste em realizar todas as operações (diferenciação, integração, etc.) em um elemento padrão (quadrilateral ou triangular) e, por meio de um mapeamento paramétrico, convertê-las para qualquer elemento do domínio. Tais operações possibilitam a montagem das matrizes do problema que dependem da natureza do mesmo e de suas equações. Utilizando-se de matrizes de conectividade e de montagem, o sistema matricial global é construído e resolvida por meio da técnica de condensação estática que desacopla alguns graus de liberdade no interior dos elementos, reduzindo o custo computacional.

Evidentemente, suprimiram-se as derivações e detalhamentos matemáticos que dizem respeito a todas as operações comentadas no parágrafo interior pois não faz parte do escopo do trabalho. O livro dos Profs. Karniadakis e Sherwin, já citado, é uma referência completa para elucidar quaisquer detalhes do método.

Tudo que foi apresentado até aqui contempla os conceitos do MEF que são utilizados no FreeFem ++ para os propósitos deste trabalho. Embora tenha mais capacidades a serem exploradas, neste trabalho o FreeFem ++ é utilizado para resolver as equações do estado estacionário dos problemas que serão estudados e apresentados no capítulo (5) enquanto, no SEMTEX, as equações serão integradas também no tempo. A seguir, apresentam-se os conceitos exclusivos do MEE.

\subsubsection{Método dos Elementos Espectrais empregado para a Solução das Equações de Navier-Stokes}

Esta seção é dedicada exclusivamente ao MEE e visa explicar sucintamente como resolver as equações de Navier-Stokes utilizando este método que é um dos propósitos desse trabalho. Vale lembrar que o loop de otimização engloba etapas de solução do escoamento e também que as equações adjuntas são derivadas das equações constitutivas. No caso desse trabalho, tratam-se das equações de 
Navier-Stokes.

Algumas particularidades dessas equações causam dificuldades na obtenção da sua solução. Citando as mais importantes: a presença de um termo não linear e o acoplamento pressão-velocidade. As maneiras de contornar esses obstáculos são, respectivamente, tratar o termo não linear de maneira explícita, além de utilizar um passo de integração adequado e a satisfação de uma condição de compatibilidade, a condição inf-sup (KARNIADAKIS; SHERWIN, 2005), pelos espaços discretos que aproximarão a solução. A descrição detalhada dessa condição pode ser encontrada na referência citada.

\subsubsection{Condições de Contorno e Avanço Temporal}

Aqui serão estabelecidas as condições de contorno para escoamentos viscosos incompressíveis que serão atribuídas nas fronteiras das malhas computacionais para solução das equações de Navier-Stokes utilizando o código SEMTEX. Nos problemas aqui estudados, serão utilizadas as seguintes condições de contorno: entrada, saída, escoamento ao longe, parede e plano de simetria, todas já exibidas na Tabela 3.1.

Para que as equações sejam resolvidas no tempo, faz-se necessária a adoção de uma discretização temporal. Visto que o MEE é um método que possibilita uma alta resolução no espaço é importante que a resolução temporal seja compatível com a alta resolução espacial (CARMO, 2005). A discretização temporal influencia diretamente na forma do sistema de equações a ser resolvido bem como está ligada na solução de problemas transientes. O método de discretização temporal proposto é o stiffly-stable que, em equações diferenciais ordinárias é baseado em diferenciação para trás (KARNIADAKIS; SHERWIN, 2005) Para ilustrar o método, reescreve-se a eq. (4.4):

$$
\tilde{\partial}_{o} \tilde{\mathbf{u}}-N(\tilde{u})=-\nabla p+\frac{1}{R e} L(\tilde{u})
$$

que está submetida à restrição:

$$
\nabla \cdot \tilde{u}=0
$$

Sendo:

$$
\left\{\begin{aligned}
N(\tilde{u})=-(\tilde{u} \nabla) \tilde{u} & =\frac{1}{2}[\tilde{u} \nabla \tilde{u}+\nabla(\tilde{u} \tilde{u})] \\
L(\tilde{u}) & =\nabla^{2} \tilde{u}
\end{aligned}\right.
$$

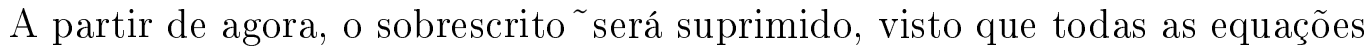


e variáveis, a partir de agora, serão adimensionais. Os termos $\mathbf{N}(\mathbf{u})$ e $\mathbf{L}(\mathbf{u})$ são, respectivamente, os termos convectivo e difusivo da equação de Navier-Stokes adimensional. No método proposto, a derivada temporal da equação (4.14) será aproximada por:

$$
\frac{\partial u}{\partial t}=\frac{1}{\Delta t}\left(\gamma_{o} u^{n+1}-\sum_{i=0}^{J-1} \alpha_{i} u^{n-i}\right)
$$

Por consistência, é necessário que:

$$
\gamma_{o}=\sum_{i=0}^{J-1} \alpha_{i}
$$

Assim, a equação (4.17) pode ser reescrita da seguinte forma:

$$
\frac{\partial u}{\partial t}=\left(\sum_{i=0}^{J-1} \alpha_{i}\left(u^{n+1}-u^{n-i}\right)\right)
$$

Seguindo essa metodologia para as equações de Navier-Stokes, obtém-se (KARNIADAKIS; SHERWIN, 2005):

$$
\frac{\gamma_{o} u^{n+1}-\sum_{q=0}^{J_{i}-i} \alpha_{q} u^{n-q}}{\Delta t}=-\nabla \bar{p}^{n+1}+\sum_{q=0}^{J_{e}-1} \beta_{q} N\left(u^{n-q}\right)+\nu L\left(u^{n+1}\right)
$$

onde $\gamma_{o}$ e $\alpha_{q}$ são os coeficientes do esquema de avanço temporal (stiffly stable) de ordem $J_{i}$ ao passo que $\beta_{q}$ são os coeficientes do esquema explícito de aproximação para $N(u)$ de ordem $J_{e}$. Dada essa formulação, a discretização temporal do problema será feita da seguinte forma, em etapas, seguindo o método splitting:

$$
\begin{gathered}
\frac{\hat{u}-u^{n}}{\Delta t}=\sum_{q=0}^{J_{e}-1} \beta_{q} N\left(u^{n-q}\right) \\
\frac{\hat{\hat{u}}-\hat{u}}{\Delta t}=-\nabla \bar{p}^{n+1} \\
\frac{\gamma_{o} u^{n+1}-\hat{\hat{u}}}{\Delta t}=\nu \nabla^{2} u^{n+1}
\end{gathered}
$$

com condições de Dirichlet em $u_{0}$ :

$$
u^{n+1}=u_{0}
$$

Os coeficientes do esquema stiffly stable são apresentados a seguir na tabela 
Tabela 4.1: Coeficientes do esquema stiffly stable para avanço temporal (KARNIADAKIS; SHERWIN, 2005)

\begin{tabular}{|c|c|c|c|}
\hline Coeficiente & $\mathbf{1}^{\text {a }}$ Ordem & $\mathbf{2}^{\text {a }}$ Ordem & $\mathbf{3}^{\text {a }}$ Ordem \\
\hline $\boldsymbol{\gamma}_{\boldsymbol{o}}$ & 1 & $3 / 2$ & $11 / 6$ \\
\hline $\boldsymbol{\alpha}_{\boldsymbol{o}}$ & 1 & 2 & 3 \\
\hline $\boldsymbol{\alpha}_{\boldsymbol{1}}$ & 0 & $-1 / 2$ & $-3 / 2$ \\
\hline $\boldsymbol{\alpha}_{\boldsymbol{2}}$ & 0 & 0 & $1 / 3$ \\
\hline $\boldsymbol{\beta}_{\boldsymbol{o}}$ & 1 & 2 & 3 \\
\hline $\boldsymbol{\beta}_{\boldsymbol{1}}$ & 0 & -1 & -3 \\
\hline $\boldsymbol{\beta}_{\boldsymbol{2}}$ & 0 & 0 & 1 \\
\hline
\end{tabular}

Os termos $\hat{u}$ e $\hat{\hat{u}}$ presentes nas eqs. (4.21) a (4.23), representam campos de velocidade intermediários. Na eq. (4.21) todos os termos são conhecidos, trata-se de uma expressão explícita. A eq. (4.22) fornece o valor de $\hat{\hat{u}}$ necessário para o cálculo de $u^{n+1}$ na eq. (4.23). Como a eq. (4.22) possui duas icógnitas, $\hat{\hat{u}} \mathrm{e}$ $\bar{p}^{n+1}$ necessita-se de uma equação adicional. Para tal, é assumido que o campo de velocidades temporário $\hat{\hat{u}}$ atende à equação da continuidade, dando origem à terceira equação:

$$
\nabla \cdot \hat{\hat{u}}=0
$$

Substituindo a eq. (4.25) em (4.22), obtém-se a seguinte equação para o campo de pressão (Equação de Poisson para a pressão):

$$
\nabla^{2} \bar{p}^{n+1}=\nabla\left(\frac{\hat{\hat{u}}}{\Delta t}\right)
$$

e para o contorno:

$$
\frac{\partial \bar{p}^{n+1}}{\partial n}=-\frac{\overrightarrow{u_{o}} \cdot n-\hat{u} \cdot n}{\Delta t}
$$

Para evitar que as soluções obtidas pelo método apresentem resultados que não traduzam corretamente o escoamento devido a erros na aplicação de condições de contorno, a correta condição de contorno para a pressão, salvo para fronteiras de saída é dada por:

$$
\frac{\partial \bar{p}^{n+1}}{\partial n}=n \cdot\left[\sum_{q=0}^{J_{e}-1} \beta_{q} N\left(u^{n-q}\right)+\frac{1}{R e} \sum_{q=0}^{J_{i}-1} \gamma_{q} L\left(u^{n+1-q}\right)\right]
$$

Nota-se que a eq.(4.28) possui termos no tempo $n+1$ que são desconhecidos. Para garantir estabilidade na solução, o termo difusivo será reescrito da seguinte forma:

$$
L(u)=\nabla^{2} u=\nabla(\nabla \cdot u)-\nabla \times(\nabla \times u)
$$


O termo $\nabla(\nabla \cdot u)$ será tratado de forma implícita enquanto o termo, $-\nabla \times$ $(\nabla \times u)$ será tratado de forma explícita. Substituindo as novas formas dos termos na eq.(4.28):

$$
\begin{array}{r}
\frac{\partial \bar{p}^{n+1}}{\partial n}=n \cdot\left[\sum_{q=0}^{J_{e}-1} \beta_{q} N\left(u^{n-q}\right)+\frac{1}{R e} \sum_{q=0}^{J_{i}-1} \gamma_{q} \nabla\left(\nabla \cdot u^{n+1-q}\right)\right. \\
\left.+\frac{1}{R e} \sum_{q=0}^{J_{i}-1} \gamma_{q}\left(-\nabla \times\left(\nabla \times u^{n-q}\right)\right)\right]
\end{array}
$$

Como o escoamento é incompressível, implica que $\nabla \cdot u^{n+1}=0$. Dessa forma, o termo $\gamma_{q} \nabla\left(\nabla \cdot u^{n+1}\right)$ se anula. Feito isso todas as velocidades no passo $n+1 \mathrm{da}$ expressão são eliminadas e o único termo desconhecido passa a ser: $\frac{\partial \bar{p}^{n+1}}{\partial n}$.

Resumindo as etapas do avanço temporal (CARMO, 2005):

1. Cálculo do campo de velocidades intermediário $\hat{u}$ com a eq. (4.21);

2. Cálculo do campo de pressões $\bar{p}^{n+1}$ com as eqs. (4.26) e (4.30);

3. De posse da pressão, faz-se o cálculo do campo intermediário $\hat{\hat{u}}$ com a eq. $(4.22)$;

4. Cálculo do campo de velocidades $u^{n+1}$ com a eq. (4.23). 


\section{ANÁlise DE SENSIBILIDADE}

Nesta etapa do trabalho, as equações adjuntas já foram obtidas (3) e obtevese uma expressão para o cálculo do gradiente de sensibilidade, uma das etapas do loop de otimização exibido na figura (1.1). Para a validação dessa expressão, é necessário escolher um caso para estudo e calcular numericamente o gradiente para uma medida de mérito desse problema. Em seguida, o valor obtido é comparado a outro valor do mesmo gradiente porém obtido analítica ou experimentalmente.

A expressão do gradiente depende de variáveis do escoamento e adjuntas que, para o cálculo numérico, são obtidas através dos códigos SEMTEX e FreeFem ++ , como já citado. Tanto as equações quanto a metodologia por trás do método empregado nos códigos foram explicadas no capítulo 4.

Inicialmente foram feitos testes com o único intuito de validar a solução adjunta e verificar se as condições de contorno eram satisfeitas. Posteriormente iniciaram-se os estudos de caso para o cálculo do gradiente de sensibilidade.

\subsection{Validação da Solução Adjunta}

Como primeiro teste do código, simulou-se um caso simples, bidimensional: escoamento ao redor de um cilindro. O principal objetivo desse primeiro teste foi obter uma solução adjunta convergida. Como parâmetros, foram escolhidos para número de Reynolds o valor $R e=30$ e, para o grau da interpolação polinomial o valor igual 8.

Para esse caso, a medida de mérito a ser calculada foi o coeficiente de arrasto sobre o cilindro, que é obtido a partir da equação abaixo:

$$
I_{o}=\bar{C}_{d}=\frac{1}{D T} \int_{0}^{T} \oint_{B} \frac{\mathbf{n} \cdot \boldsymbol{\sigma} \cdot \mathbf{e}_{x}}{q_{\infty}} \mathrm{d} S \mathrm{~d} t
$$

onde $\mathbf{e}_{x}$ é a direção paralela ao escoamento, ao longo do eixo $x, B$ representa 
a superfície do cilindro e $D$ é seu diâmetro. As condições de contorno foram impostas nos problemas físico e adjunto. A condição de parede será expressa, na sua forma adimensional, por:

$$
\tilde{\boldsymbol{\varphi}}=-\frac{\partial \tilde{G}}{\partial \tilde{\mathcal{F}}}\left|\frac{\mathrm{d} S^{\prime}}{\mathrm{d} S}\right|_{B}
$$

onde $\tilde{G}$ e $\left|\mathrm{d} S^{\prime} / \mathrm{d} S\right|$ são assumidos como adimensionais, e $\tilde{\mathcal{F}}$ é definido como $\tilde{\mathcal{F}}=$ $\mathcal{F} / \rho U_{\infty}^{2}$. A condição adjunta de saída ficará na forma:

$$
\left\{\begin{array}{l}
\frac{\partial \tilde{\varphi}}{\partial n}=-\operatorname{Re}(\tilde{\mathbf{u}} \cdot \mathbf{n}) \tilde{\varphi} \\
\tilde{\Theta}=0
\end{array}\right.
$$

No caso particular do escoamento ao redor do cilindro, a condição de parede expressa na equação (5.2) passa a ser dada por:

$$
\tilde{\varphi}=-2 \mathbf{e}_{x}\left|\frac{\mathrm{d} S^{\prime}}{\mathrm{d} S}\right|_{B}
$$

Com as condições de contorno já definidas deseja-se validar a solução adjunta obtida pelo SEMTEX com o intuito de validar o código para as aplicações desejadas neste trabalho. Para tal, escolheu-se um caso teste, fornecido pelos autores do código, que consiste em um escoamento ao redor de um cilindro. O objetivo é obter uma solução covnergida para as equações adjuntas dadas as condições de contorno apresentadas anterioemente. A seguir estão mostradas figuras das soluções do escoamento e adjunta:
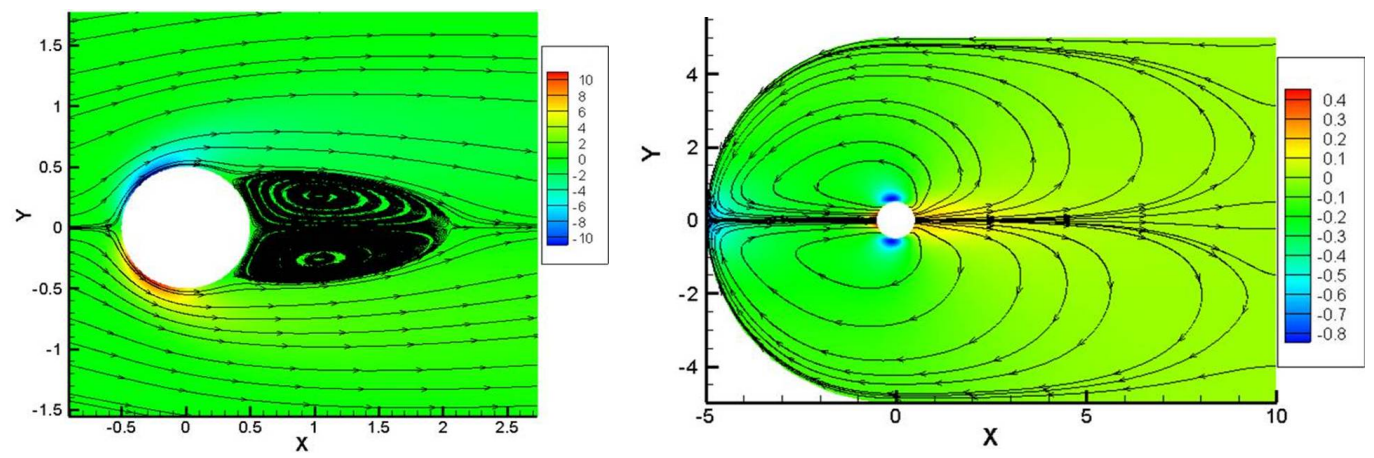

Figura 5.1: Escoamento em torno de um cilindro circular - Soluções para o escoamento 2-D e adjunta utilizando o código SEMTEX, $R e=30$, P.50.

À esquerda, detalhe do escoamento em torno do cilindro com linhas de corrente, e ao fundo a escala de cores corresponde à intensidade da vorticidade $\omega_{z}$. À direita, a solução adjunta com linhas de corrente de velocidade adjunta $\varphi_{k}$, e ao fundo a escala de cores corresponde à intensidade da pressão adjunta $\Theta$. Nota- 
se, observando a figura à direita, que foi obtida uma solução convergida para as equações adjuntas.

\subsection{Cálculo de sensibilidades não geométricas}

Uma vez que bem sucedeu-se a obtenção de uma solução adjunta convergida utilizando ambos os códigos propostos, é possível partir efetivamente para o cálculo dos gradientes de sensibilidade. Recuperando a figura (1.1) após a simulação do escoamento verifica-se se a medida de mérito escolhida já está otimizada. Se a resposta for negativa, é obtida a solução adjunta do problema e em seguida calcula-se o gradiente de sensibilidade da medida de mérito em relação a um ou mais parâmetros de projeto para obter uma direção de variação dos parâmetros de forma a otimizar essa medida de mérito.

Visto que o objetivo desse trabalho é validar a metodologia proposta, os casos iniciais de validação consistem em problemas clássicos de escoamentos laminares nos quais será calculada a sensibilidade do coeficiente de arrasto $C_{d}$ em relação à velocidade do escoamento ao longe $U_{\infty}$. A escolha de um desses casos se dá principalmente pelo fato do mesmo possuir uma expressão analítica (ou semiempírica), embora aproximada, para o cálculo de $C_{d}$ em relação a $U_{\infty}$. Desse modo também é possível obter uma expressão analítica para o gradiente. Foram escolhidos alguns escoamentos internos como o escoamento através de um canal bidimensional com gradiente de pressão (escoamento de Couette) e escoamento através de um canal com variação de diâmetro. As etapas de estudo consistem no cálculo dos gradientes via método adjunto, via diferenças finitas e via expressão analítica (quando aplicável), sendo as duas últimas para fins de comparação com a primeira.

\subsubsection{Escoamento Através de Um Canal Bidimensional - Escoamento de Couette}

\subsubsection{Descrição do Problema}

O primeiro caso de validação corresponde a um escoamento através de um canal bidimensional formado por duas placas paralelas e infinitas. Um esquema do problema é apresentado a seguir:

Neste problema é importante atentar para a escala de comprimento utilizada para o cálculo do Re. Para o canal, essa escala corresponde ao diâmetro hidráulico 


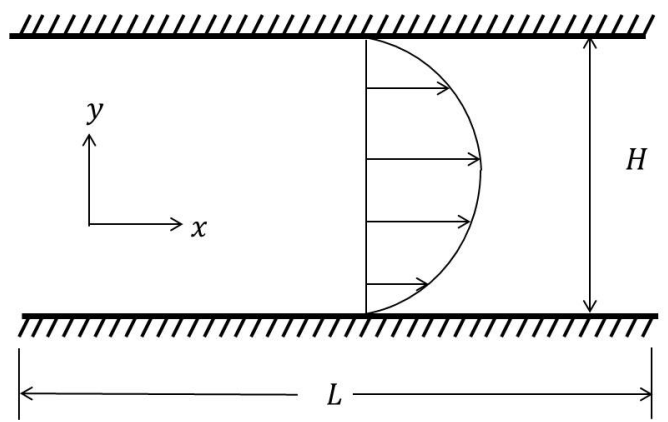

Figura 5.2: Esquema do escoamento através de um canal bidimensional, de comprimento $L$, formada por duas placas paralelas e infinitas, distantes $H$ uma da outra

$\left(D_{H}\right)$ da seção. Neste problema o diâmetro hidráulico é calculado por:

$$
D_{H}=2 . H
$$

\subsubsection{Obtenção da Expressão Analítica para o Cálculo do Gradiente}

A solução analítica para esse escoamento é trivial de ser obtida. Aplica-se a equação de conservação da quantidade de movimento na direção $x$ e adotam-se as seguintes hipóteses: regime estacionário e escoamento plenamente desenvolvido na maior parte da passagem, ou $u=u(y)$. Com tais hipóteses, simplifica-se a equação de conservação da quantidade de movimento e encontra-se o perfil parabólico de velocidades que, integrado na direção $y$ fornece a vazão média com a qual se calcula a velocidade média do escoamento. Calculam-se por fim a tensão de cisalhamento nas paredes, o coeficiente de fricção e $C_{d}$. A expressão obtida é dada por (WHITE, 2006):

$$
C_{d}=\frac{48}{R e}
$$

Sabendo-se que $R e=\frac{U_{\infty} D_{H}}{\nu}$, é possível obter uma expressão para $C_{d} \mathrm{em}$ função das três escalas de $R e$ :

$$
C_{d}=\frac{48 \nu}{U_{\infty} D_{H}}
$$

Derivando a equação acima, obtém-se a expressão analítica para o gradiente de $C_{d}$ em relação a $U_{\infty}$ :

$$
\frac{d C_{d}}{d U_{\infty}}=-\frac{48 \nu}{U_{\infty}^{2} D_{H}}=-\frac{48}{U_{\infty} R e}
$$




\subsubsection{Cálculo do Gradiente via Diferenças Finitas Centradas}

A expressão para cálculo do gradiente via diferenças finitas centradas é amplamente difundida na literatura. Para o caso específico desse problema (e de todos os outros que calculem a sensibilidade de $C_{d}$ em relação a $U_{\infty}$ ):

$$
\frac{\mathrm{d} C_{d}}{\mathrm{~d} U_{\infty}}=\frac{\left.C_{d}\right|_{U_{\infty}+\delta U_{\infty}}-\left.C_{d}\right|_{U_{\infty}-\delta U_{\infty}}}{2\left(\delta U_{\infty}\right)}+\mathcal{O}\left(\delta U_{\infty}\right)^{2}
$$

\subsubsection{Cálculo do Gradiente via Método Adjunto}

No capítulo 3 foi apresentado o cálculo do gradiente adjunto nas eqs. (5.25) e (3.121). Adimensionalizando essas expressões, obtém-se a expressão para o cálculo do gradiente de sensibilidade via método adjunto de uma medida de mérito em relação a um parâmetro $\alpha$ :

$$
\begin{aligned}
\tilde{T}\langle\delta \tilde{\alpha}, \tilde{\mathbf{a}}\rangle & =\int_{0} \oint_{B} \tilde{G} \delta\left|\frac{d S^{\prime}}{d S}\right| \mathrm{d} \tilde{S} \mathrm{~d} \tilde{t}+\int_{\tilde{\Omega}} \frac{\tilde{\Theta}}{J}\left[\delta\left(J \beta_{q^{\prime}}^{k}\right) \tilde{u}^{q^{\prime}}\right]_{, k} \mathrm{~d} \tilde{\Omega}+ \\
& -\int_{\tilde{\Omega}} \frac{\tilde{\varphi}_{r^{\prime}}}{J}\left\{\partial_{o}\left(\delta J \tilde{u}^{r^{\prime}}\right)+\left[\delta\left(J \beta_{n^{\prime}}^{j}\right)\left(\tilde{u}^{r^{\prime}} \tilde{u}^{n^{\prime}}+g^{r^{\prime} n^{\prime}} \tilde{p}-\frac{1}{R e} \tilde{u}_{, p^{\prime}}^{r^{\prime}} g^{p^{\prime} n^{\prime}}\right)\right]_{, j}\right\} \mathrm{d} \tilde{\Omega} \\
& +\int_{0}^{\oint_{\tilde{S}_{i}}}\left[-\frac{1}{R e}\left(\left.\tilde{\varphi}_{i}\right|_{j}+\left.\tilde{\varphi}_{j}\right|_{i}\right) n^{j}+\tilde{\Theta} n_{i}\right] \frac{\partial \tilde{f}^{i}}{\partial \tilde{\mathbf{a}}^{k}} \delta \tilde{\mathbf{a}}^{k} \mathrm{~d} \tilde{S} \mathrm{~d} \tilde{t}
\end{aligned}
$$

Visto que o parâmetro sobre o qual será calculado o gradiente é a velocidade na fronteira de entrada, tem-se que $\tilde{\mathbf{u}}=\tilde{\mathbf{f}}\left(U_{\infty}\right)$ o que leva a:

$$
\frac{\partial \tilde{f}}{\partial \tilde{\alpha}}=1
$$

Definem-se agora as escalas adimensionais do problema:

$$
\tilde{D_{H}}=\frac{D_{H}}{L_{R}} \quad ; \quad \tilde{U}_{\infty}=\frac{U}{U_{R}}
$$

onde $L_{R}=D_{H}$. Utilizando as escalas adimensionais exibidas na equação (5.12) obtém-se:

$$
\tilde{x}^{i^{\prime}}=\tilde{D}_{H} \tilde{x}^{i}
$$

Os operadores da transformação e parâmetros da métrica são dados por:

$$
\beta_{j^{\prime}}^{i}=\frac{1}{\tilde{D_{H}}} \delta_{j^{\prime}}^{i} \quad ; \quad \beta_{i}^{j^{\prime}}=\tilde{D_{H}} \delta_{i}^{j^{\prime}} \quad ; \quad J=\tilde{D_{H}}{ }^{2} \quad ; \quad\left|\frac{\mathrm{d} S^{\prime}}{\mathrm{d} S}\right|=\tilde{D_{H}}
$$

baseado no conteúdo disponível nos apêndices A e B. Variacionando os termos 
acima:

$\delta \beta_{j^{\prime}}^{i}=-\frac{\delta \tilde{D_{H}}}{{\tilde{D_{H}}}^{2}} \delta_{j^{\prime}}^{i} \quad ; \quad \delta\left(J \beta_{j^{\prime}}^{i}\right)=\delta \tilde{D_{H}} \delta_{j^{\prime}}^{i} \quad ; \quad \delta J=2 \tilde{D_{H} \delta \tilde{D_{H}}} \quad ; \quad \delta\left|\frac{\mathrm{d} S^{\prime}}{\mathrm{d} S}\right|=\delta \tilde{D_{H}}$

Substituindo todas essas expressões na eq. (5.10):

$$
\begin{aligned}
\tilde{T}\langle\delta \tilde{\alpha}, \tilde{\mathbf{a}}\rangle & =\int_{0} \oint_{B} \tilde{G} \frac{\partial \tilde{D_{H}}}{\partial \tilde{\mathbf{a}}} \delta \tilde{\mathbf{a}} \mathrm{d} \tilde{S} \mathrm{~d} \tilde{t}+\int_{\tilde{\Omega}} \frac{\tilde{\Theta}}{\tilde{D}_{H}{ }^{2}} \frac{\partial \tilde{D_{H}}}{\partial \tilde{\mathbf{a}}} \delta \tilde{\mathbf{a}}\left[\tilde{u}^{k}\right]_{, k} \mathrm{~d} \tilde{\Omega}+ \\
& -\int_{\tilde{\Omega}} \frac{\tilde{\varphi}_{r^{\prime}}}{{\tilde{D_{H}}}^{2}}\left\{2 \tilde{D}_{H} \partial_{o} \tilde{u}^{r^{\prime}}+\left[\left(\tilde{u}^{r^{\prime}} \tilde{u}^{n^{\prime}}+g^{r^{\prime} n^{\prime}} \tilde{p}-\frac{1}{R e} \tilde{u}_{, p^{\prime}}^{r^{\prime}} g^{p^{\prime} n^{\prime}}\right)\right]_{, n^{\prime}}\right\} \frac{\partial \tilde{D_{H}}}{\partial \tilde{\mathbf{a}}} \delta \tilde{\mathbf{a}} \mathrm{d} \tilde{\Omega} \\
& +\int_{0} \oint_{\tilde{S}_{i}}\left[-\frac{1}{R e}\left(\left.\tilde{\varphi}_{i}\right|_{j}+\left.\tilde{\varphi}_{j}\right|_{i}\right) n^{j}+\tilde{\Theta} n_{i}\right] \frac{\partial \tilde{f}^{i}}{\partial \tilde{\mathbf{a}}} \delta \tilde{\mathbf{a}} \mathrm{d} \tilde{S} \mathrm{~d} \tilde{t}
\end{aligned}
$$

Para esse problema, $\tilde{D_{H}}=1$ na solução base. Como $U_{R}=U_{\infty}$ é o único parâmetro de controle e dado que a solução satisfaz as equações de Navier-Stokes, chega-se na expressão final do gradiente adjunto para esse problema:

$$
\begin{aligned}
\tilde{T}\left\langle\tilde{\alpha}, \delta U_{\infty}\right\rangle & =\int_{0} \oint_{B} \tilde{G} \frac{\partial \tilde{D_{H}}}{\partial U_{\infty}} \delta U_{\infty} \mathrm{d} \tilde{S} \mathrm{~d} \tilde{t}-\int_{\tilde{\Omega}} \tilde{\varphi}_{r^{\prime}} \partial_{o} \tilde{u}^{r^{\prime}} \frac{\partial \tilde{D_{H}}}{\partial U_{\infty}} \delta U_{\infty}, \mathrm{d} \tilde{\Omega}+ \\
& +\int_{0} \oint_{\tilde{S}_{i}}\left[-\frac{1}{R e}\left(\left.\tilde{\varphi}_{i}\right|_{j}+\left.\tilde{\varphi}_{j}\right|_{i}\right) n^{j}+\tilde{\Theta} n_{i}\right] \frac{\partial \tilde{f}}{\partial U_{\infty}} \delta U_{\infty} \mathrm{d} \tilde{S} \mathrm{~d} \tilde{t}
\end{aligned}
$$

Para esse caso específico, a primeira integral será nula pois não há dependência de $D_{H}$ em relação a $U_{\infty}$ ao passo que a segunda integral será nula pois o escoamento é estacionário e, consequentemente, o termo de variação temporal será o canal bidimensional, na sua expressão mais simplificada é dado por:

$$
\frac{\mathrm{d} C_{d}}{\mathrm{~d} U_{\infty}}=\int_{\tilde{S}_{i}} \mathbf{n} \cdot\left\{-\frac{1}{R e}\left[\nabla \varphi+(\nabla \varphi)^{T}\right]+\Theta \mathbb{I}\right\} \frac{\partial \tilde{f}}{\partial U_{\infty}} d \tilde{S}
$$

onde $\tilde{S}_{i}$ representa a fronteira de entrada. Nota-se que a única integral restante neste caso é a integral na fronteira de entrada que advém da formulação do Cacuci (seção 3.3 em diante) cujo objetivo é, justamente, calcular sensibilidades em relação a parâmetros não geométricos.

O cálculo desse gradiente adjunto é feito em uma rotina externa ao código utilizado para a solução do escoamento e das equações adjuntas. A rotina foi elaborada em Matlab e a integração é efetuada utilizando Quadratura de Gauss- 
Lobatto-Legendre. Esse gradiente, calculado em relação a um parâmetro não geométrico, permite verificar o quanto a solução (ótima ou não) é sensível em relação a esse parâmetro. Como já mencionado, o problema apresentado é um caso simples com fins exclusivos de validação do método. Em problemas mais complexos, com mais parâmetros de controle, o cálculo do gradiente permite análises de sensibilidade de modo a fornecer quais parâmetros de controle influenciam mais uma determinada medida de mérito do problema.

\subsubsection{Soluções do Escoamento e Adjunto - SEMTEX}

Aqui serão apresentadas as soluções das equações do escoamento e adjuntas utilizando o código SEMTEX. A malha utilizada para a simulação das soluções do escoamento e adjunta está exibida a seguir. É uma malha elaborada no software comercial Gambit, possui 800 elementos quadrilaterais. O teste de convergência para obtenção dessa malha está disponível no apêndice C.

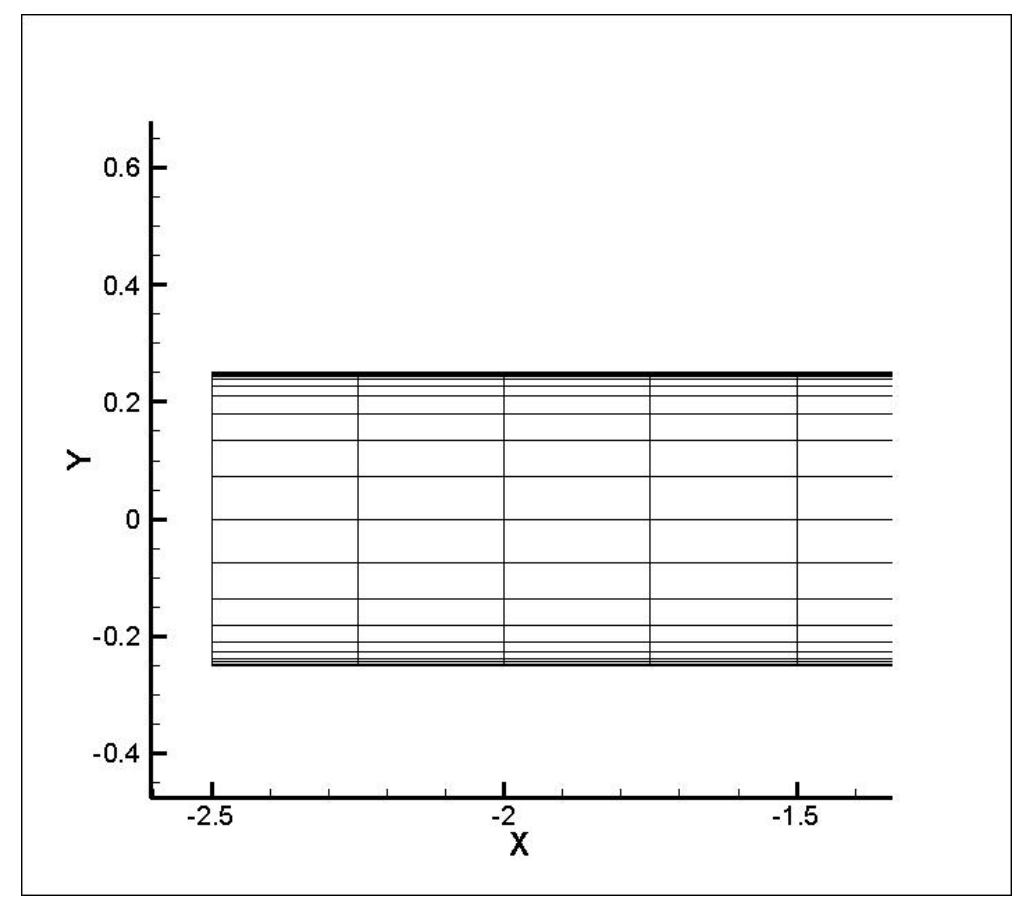

Figura 5.3: Malha Computacional utilizada nas simulações para o escoamento e adjunto

A malha está definida em $-2.5 \leq x \leq 30$ e tem espessura igual a 0,5 unidades. Para melhor visualização foi mostrado apenas o início da malha. Entre $-2.5 \leq x \leq 0$ foi utilizado um artifício para evitar singularidades de condições de contorno. Dessa forma, no intervalo dado foram atribuídas condições de contorno de simetria nas fronteiras inferior e superior. A partir de $x=0$ tem início o trecho da passagem, com condições de contorno de parede. Caso tal artifício não fosse 
adotado, no ponto de intersecção das fronteiras inferior e esquerda, haveria duas atribuições de valor distintas para a velocidade horizontal $(u=0$ para condição de parede e $u=1$ para condição de entrada), originando singularidades. Dessa forma, para valores de $x \geq 0$ foram atribuídas condições de contorno de parede para as fronteiras inferior e superior e condição de saída na fronteira direita. Abaixo, na figura (5.4) estão as soluções do escoamento e adjunta para $R e=30$, grau de polinômio $P=10$ e 7000 passos no tempo com magnitude $\Delta t=10^{-3}$ cada um.
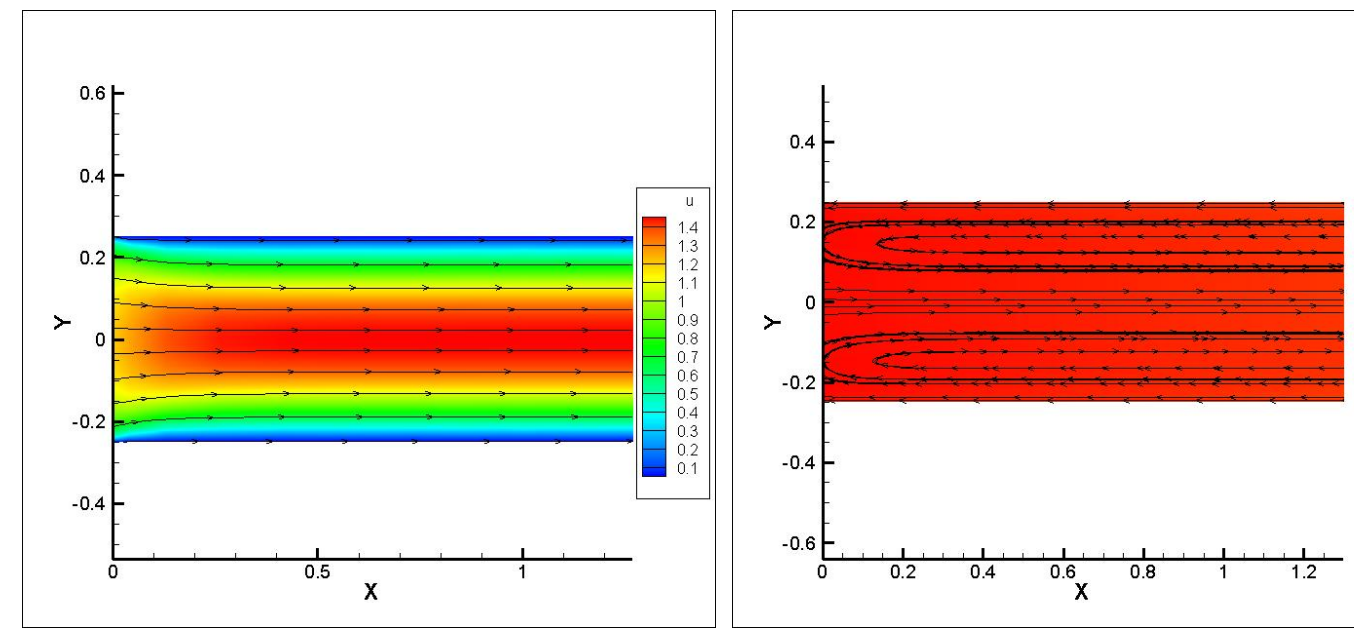

Figura 5.4: Escoamento através de um canal bidimensional - Detalhes das soluções para o escoamento $2-\mathrm{D}$ e adjunta, $R e=30$, grau do polinômio $\mathrm{P}=10$.

À esquerda, solução do escoamento com linhas de corrente, e ao fundo a escala de cores corresponde à intensidade da velocidade horizontal $u$. À direita, a solução adjunta com linhas de corrente de velocidade adjunta $\varphi_{k}$, e ao fundo a escala de cores corresponde à intensidade da pressão adjunta $\Theta$.

\subsubsection{Cálculo e Comparativo de Gradientes - SEMTEX}

Velocidade na entrada $U_{\infty}=1,0 \mathrm{~m} / \mathrm{s}$

Na figura 5.5 estão exibidos os gradientes calculados para diferentes valores de $R e$ e com velocidade do escoamento na entrada $U_{\infty}=1,0 \mathrm{~m} / \mathrm{s}$ via método adjunto e via diferenças finitas, para o código SEMTEX. Os parâmetros utilizados nas simulações computacionais foram apresentados anteriormente. O gráfico a seguir e os subsequentes possuem 16 pontos cada um divididos igualmente entre duas curvas representadas por cores distintas. Para a obtenção de cada ponto foram necessárias 2 simulações, o que faz com que ao todo tenham sido necessárias 32 simulações computacionais para a geração dos resultados expressos nos gráficos a seguir. 


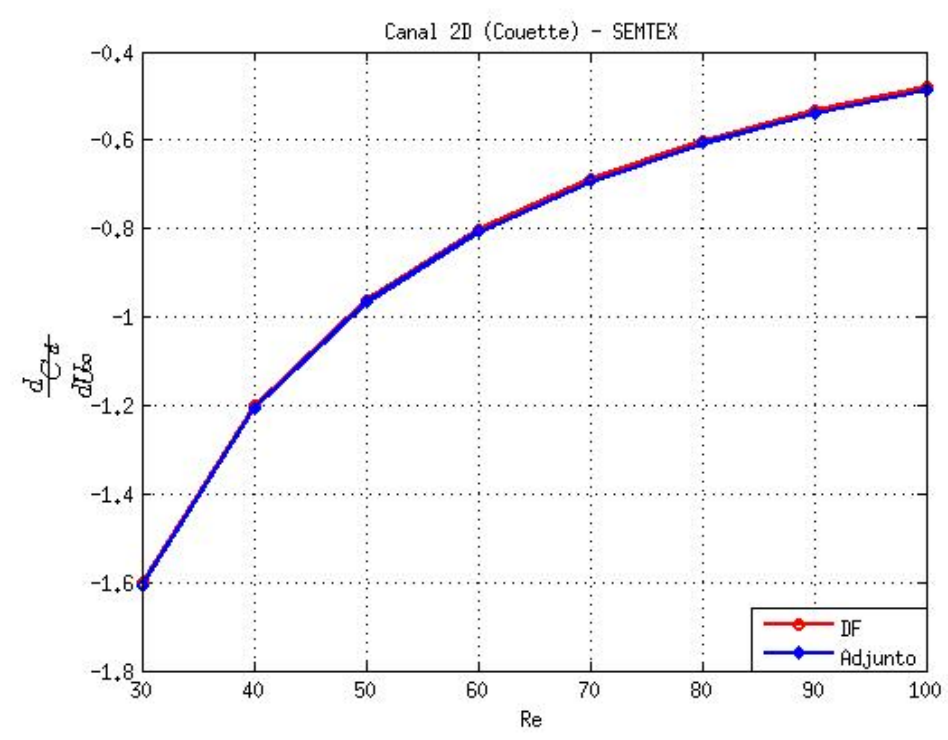

Figura 5.5: Gráfico comparativo dos gradientes calculados via método adjunto (azul) e via diferenças finitas (vermelho) para diferentes valores de $R e$

Nota-se que os gradientes calculados via método adjunto e via diferenças finitas apresentam valores muito próximos (erro inferior a 1.5\%) validando, assim, a expressão para o gradiente de sensibilidade em relação a um parâmetro não geométrico (neste caso, a velocidade). Como citado, este problema possibilita o cálculo do mesmo gradiente de maneira analítica e assim, é possível comparar o gradiente adjunto com o gradiente analítico, o que está apresentado na figura (5.6)

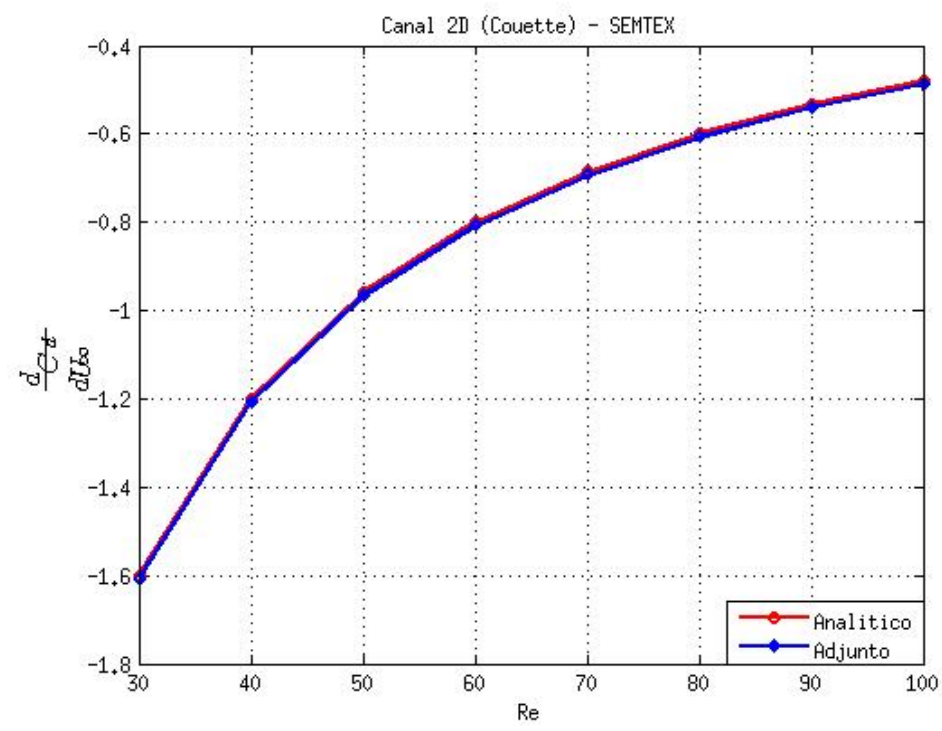

Figura 5.6: Gráfico comparativo dos gradientes calculados via método adjunto (azul) e analiticamente (vermelho) para diferentes valores de $R e$ 
Novamente, observa-se grande proximidade entre os gradientes adjunto e analítico (erro inferior a 1,5\%). Optou-se por apresentar esse comparativo separadamente do gráfico que contém o gradiente calculado via diferenças finitas, devido à proximidade das curvas o que dificultaria a visualização.

$\underline{\text { Velocidade na entrada } U_{\infty}=2,0 \mathrm{~m} / \mathrm{s}}$

Na figura 5.7 estão exibidos os gradientes calculados de maneira semelhante ao caso anterior porém com velocidade na entrada igual a $2,0 \mathrm{~m} / \mathrm{s}$. Nota-se que

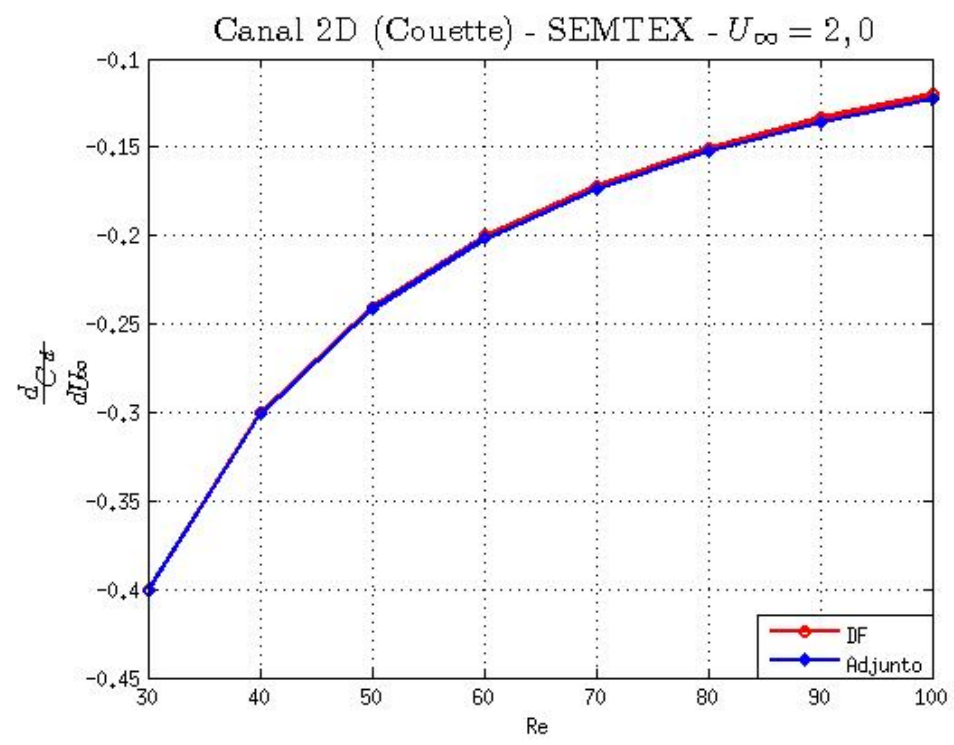

Figura 5.7: Gráfico comparativo dos gradientes calculados via método adjunto (azul) e via diferenças finitas (vermelho) para diferentes valores de $R e$ com velocidade na entrada $U_{\infty}=2,0 \mathrm{~m} / \mathrm{s}$

também neste caso os gradientes calculados via método adjunto e via diferenças finitas apresentam valores muito próximos (erro inferior a 2.0\%). Comparando, agora, com a expressão analítica para o gradiente: 


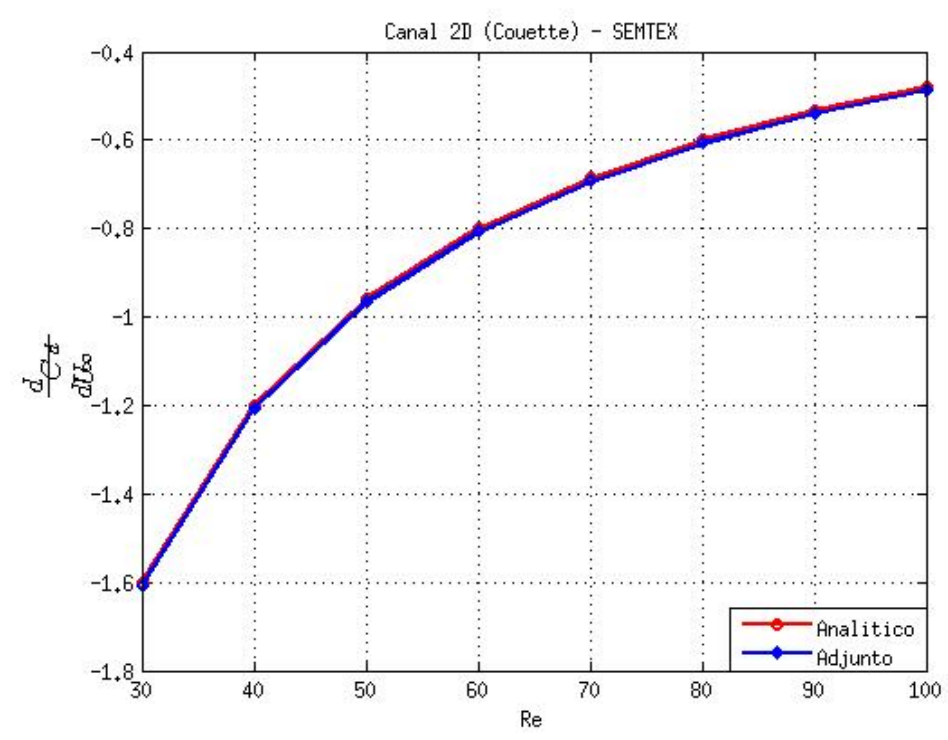

Figura 5.8: Gráfico comparativo dos gradientes calculados via método adjunto (azul) e analiticamente (vermelho) para diferentes valores de $R e$ com velocidade na entrada $U_{\infty}=2,0 \mathrm{~m} / \mathrm{s}$

Novamente nota-se a proximidade entre os valores dos gradientes calculados por duas vias distintas (erro inferior a $2.0 \%$ ).

\subsubsection{Soluções do Escoamento e Adjunto - FreeFem ++}

Nesta seção serão apresentadas as soluções das equações do escoamento e adjuntas utilizando o código FreeFem ++ . A malha utilizada para a simulação das soluções do escoamento e adjunta está exibida abaixo. A mesma possui 32414 elementos triangulares. O teste de convergência para obtenção dessa malha também está disponível no apêndice C.

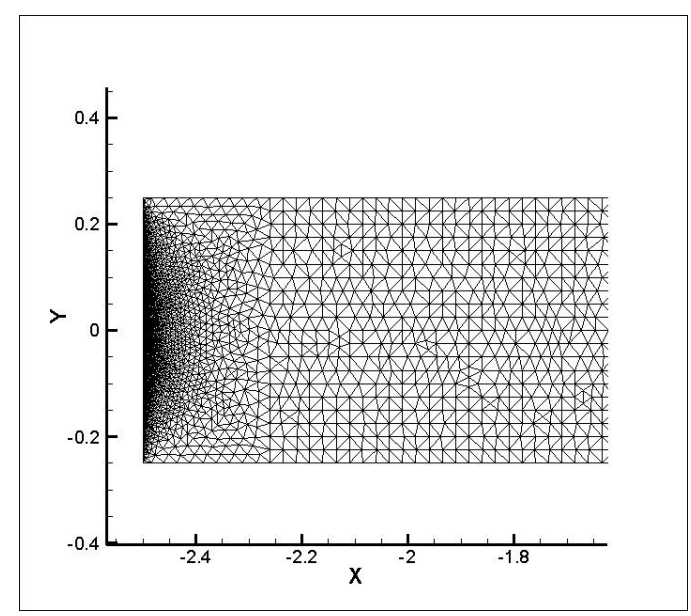

Figura 5.9: Malha Computacional utilizada nas simulações para o escoamento e adjunto 
A malha utilizada neste caso está definida em $-2.5 \leq x \leq 30$. Para melhor visualização, novamente, foi mostrado apenas o início da malha. Neste caso utilizou-se o mesmo artifício computacional utilizado no SEMTEX ao atribuir condição de contorno de simetria nos trechos superior e inferior da malha entre a fronteira de entrada e o início do canal. Abaixo, na figura (5.10) estão as soluções do escoamento e adjunta para $R e=30$ e grau de polinômio $P=4$. Como no FreeFem ++ resolve-se somente o estado estacionário, não escolhe-se magnitude do passo de integração no tempo nem a quantidade de passos.
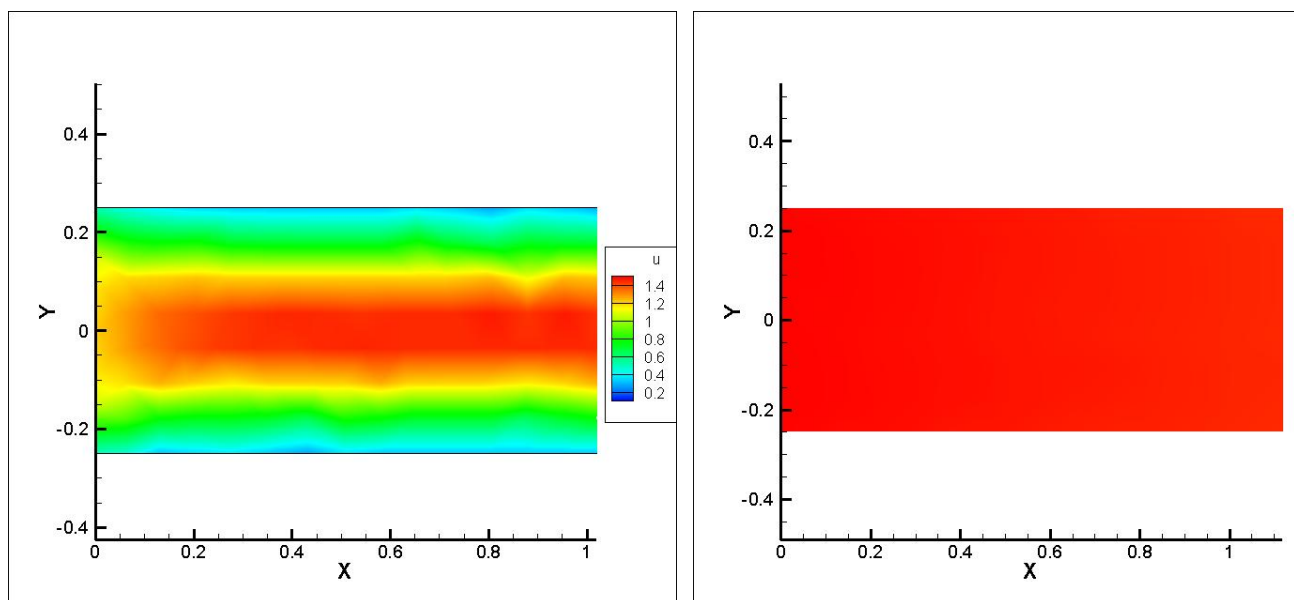

Figura 5.10: Escoamento através de um canal bidimensional - Detalhes das soluções para o escoamento $2-\mathrm{D}$ e adjunta, $R e=30$, grau de polinômio $\mathrm{P}=4$.

À esquerda, solução do escoamento com escala de cores ao fundo correspondente à intensidade da velocidade horizontal $u$. À direita, a solução adjunta com escala de cores ao fundo correspondente à intensidade da pressão adjunta $\Theta$.

\subsubsection{Cálculo e Comparativo de Gradientes - FreeFem ++}

$\underline{\text { Velocidade na entrada } U_{\infty}=1,0 \mathrm{~m} / \mathrm{s}}$

Na figura (5.11) estão exibidos os gradientes calculados para diferentes valores de $R e$ e com velocidade do escoamento na entrada $U_{\infty}=1,0 \mathrm{~m} / \mathrm{s}$ via método adjunto e via diferenças finitas centradas, para o código FreeFem ++ . 


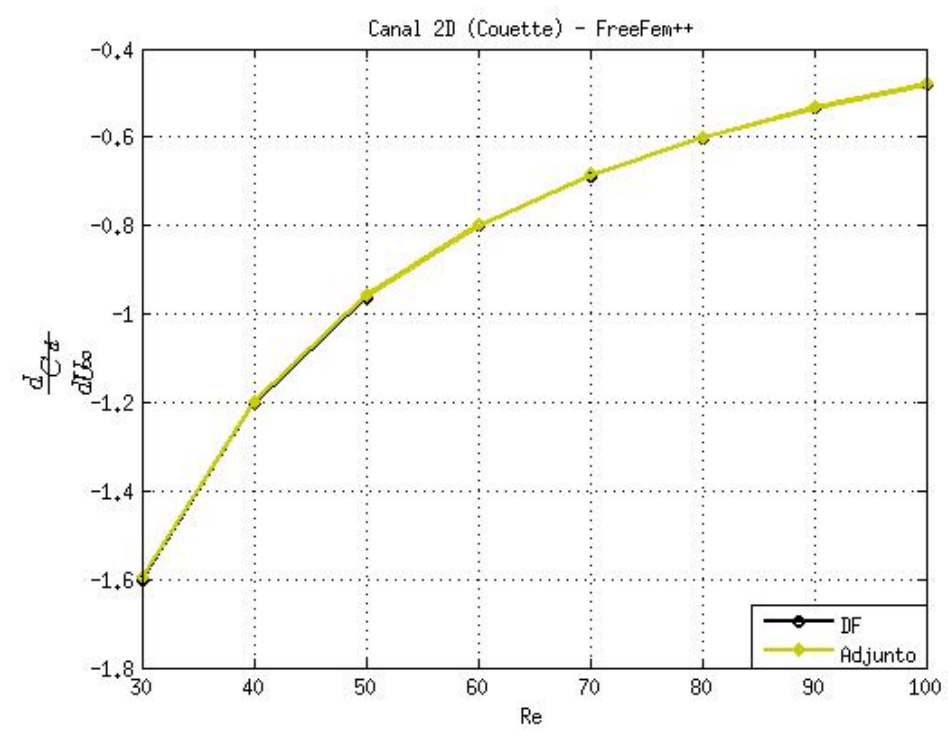

Figura 5.11: Gráfico comparativo dos gradientes calculados via método adjunto (amarelo) e via diferenças finitas (preto) para diferentes valores de $R e$

Neste caso, nota-se uma maior proximidade dos valores dos gradientes calculados via método adjunto e diferenças finitas (erro inferior a 0,5\%) em comparação com os valores calculados utilizando o SEMTEX. Aqui, novamente, compara-se o gradiente adjunto com o gradiente analítico, que está apresentado na figura (5.12) Novamente, observa-se grande proximidade entre os gradientes adjunto e

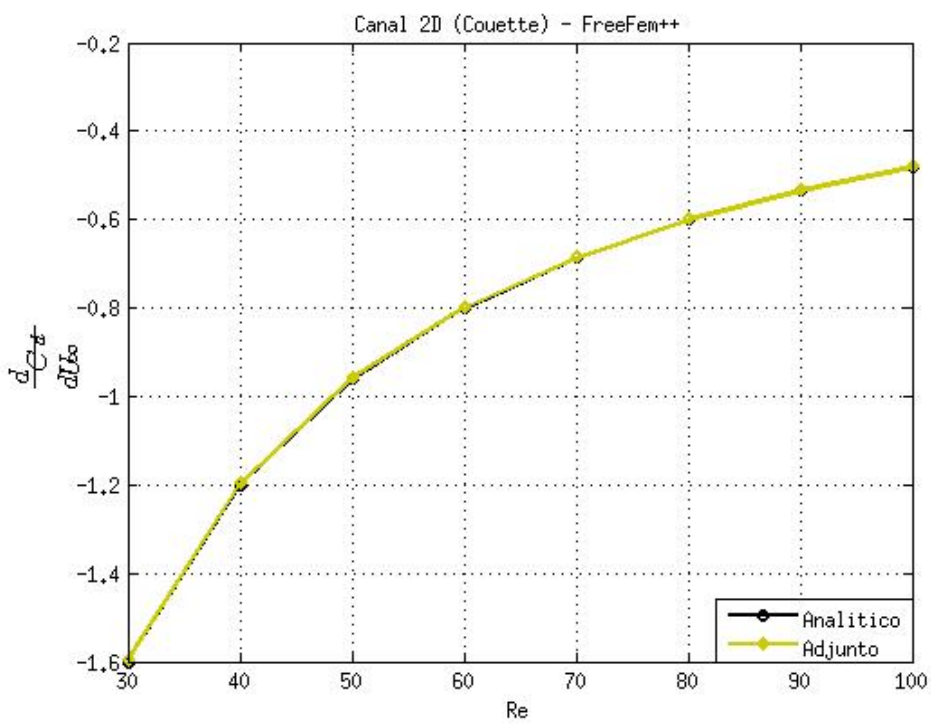

Figura 5.12: Gráfico comparativo dos gradientes calculados via método adjunto (amarelo) e analiticamente (preto) para diferentes valores de $R e$

analítico mesmo com a imprecisão relatada anteriormente (erro inferior a 0,5\%). 
$\underline{\text { Velocidade na entrada } U_{\infty}=2,0 \mathrm{~m} / \mathrm{s}}$

Repete-se agora, para o FreeFem ++ o mesmo teste com velocidade na fronteira de entrada igual a $2,0 \mathrm{~m} / \mathrm{s}$

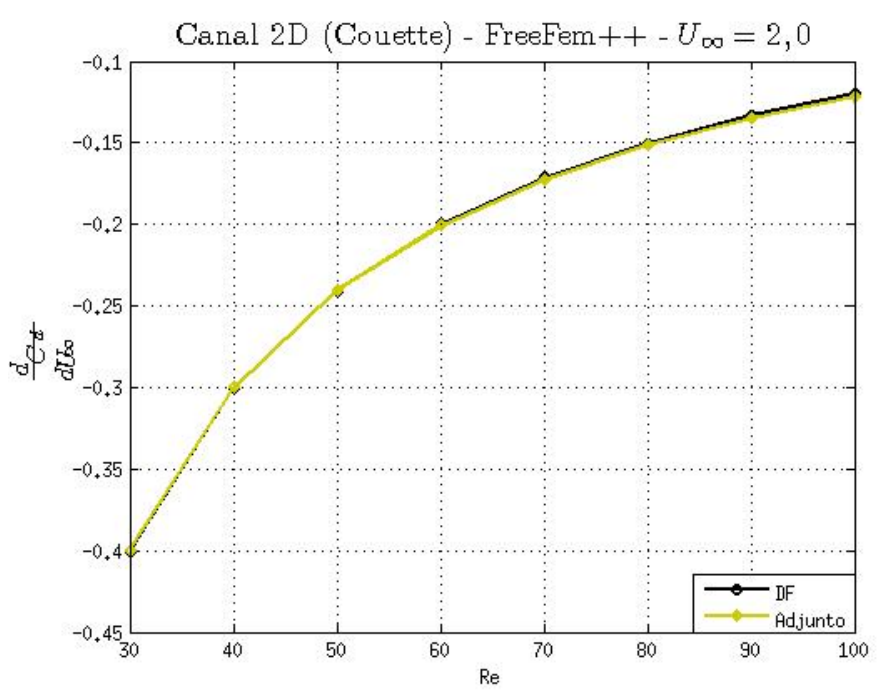

Figura 5.13: Gráfico comparativo dos gradientes calculados via método adjunto (amarelo) e via diferenças finitas (preto) para diferentes valores de $R e$ com velocidade na entrada $U_{\infty}=2,0 \mathrm{~m} / \mathrm{s}$

Verifica-se a proximidade dos valores calculados via método adjunto e via diferenças finitas (erro inferior a 1,5\%). Aqui, novamente, compara-se o gradiente adjunto com o gradiente analítico, o que está apresentado na figura 5.14

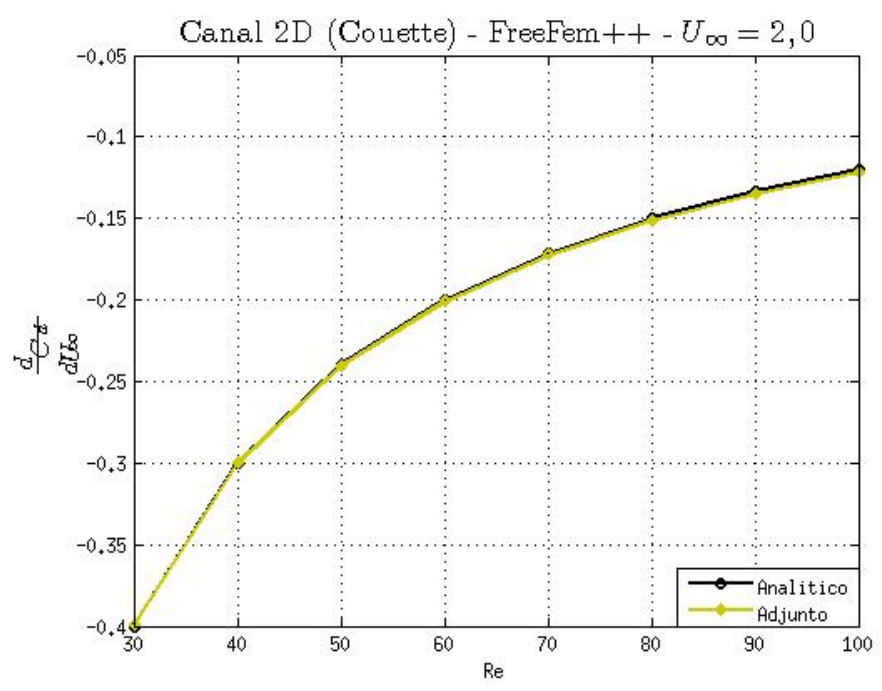

Figura 5.14: Gráfico comparativo dos gradientes calculados via método adjunto (amarelo) e analiticamente (preto) para diferentes valores de $R e$ com velocidade na entrada $U_{\infty}=2,0 \mathrm{~m} / \mathrm{s}$ 
Finalmente, observa-se grande proximidade entre os gradientes adjunto e analítico mesmo com a imprecisão relatada anteriormente (erro inferior a 1,5\%).

\subsubsection{Comparativo de Gradientes - SEMTEX x FreeFem ++}

Aqui é apresentado um gráfico comparativo entre os gradientes calculados via método adjunto em ambos os códigos computacionais.

$\underline{\text { Velocidade na entrada } U_{\infty}=1,0 \mathrm{~m} / \mathrm{s}}$ Nota-se que os valores calculados pelos

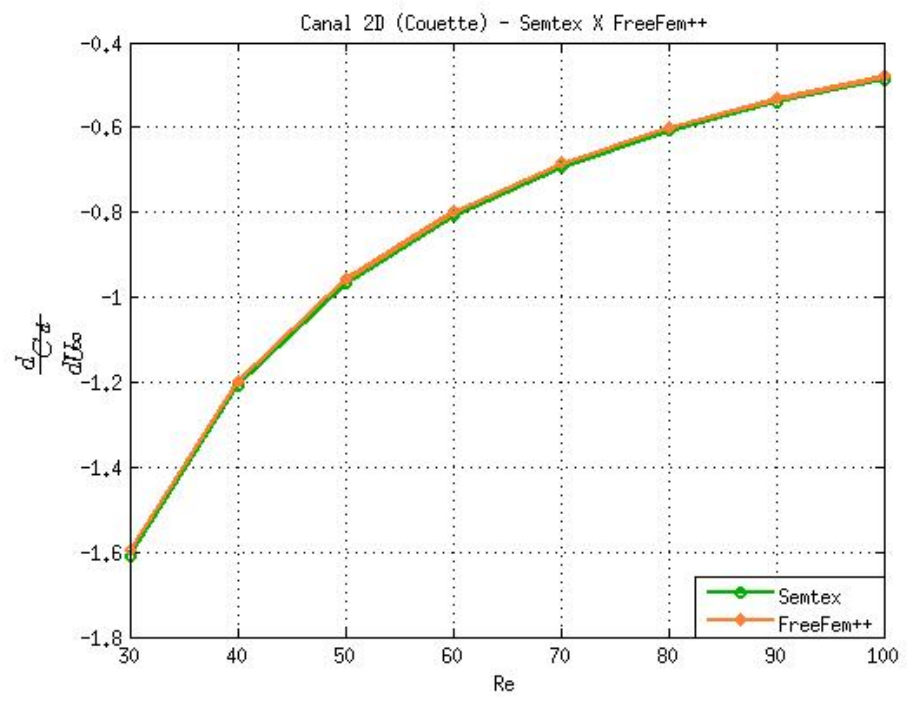

Figura 5.15: Gráfico comparativo dos gradientes calculados via método adjunto nos códigos Semtex (verde) e FreeFem ++ (laranja) para diferentes valores de $R e$

dois códigos são bastante próximos (diferença de $0,5 \%$ ) o que mostra a capacidade de ambos os códigos, mesmo com suas diferenças, serem utilizados para o cálculo de sensibilidades utilizando o método adjunto. Isso só é possível, como já mencionado, pelo fato da formulação contínua do método estar sendo utilizada.

$\underline{\text { Velocidade na entrada } U_{\infty}=2,0 \mathrm{~m} / \mathrm{s}}$

Faz-se a mesma comparação do item anterior para os testes com velocidade na entrada igual a $2,0 \mathrm{~m} / \mathrm{s}$ Aqui observa-se também a proximidade entre os gradientes calculados através dos dois códigos (erro inferior a 1,0\%). 


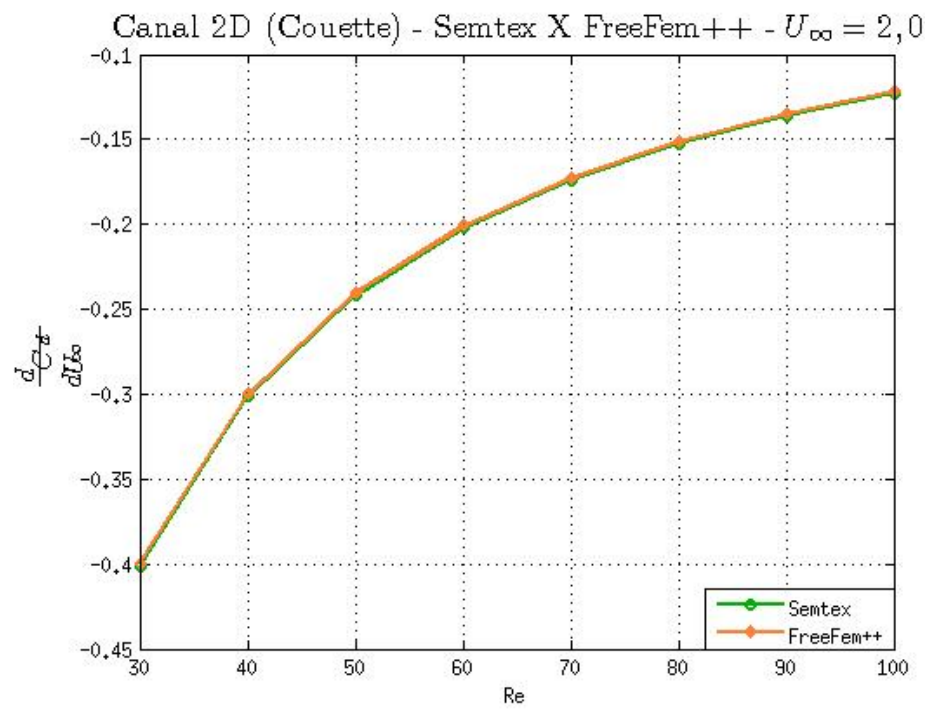

Figura 5.16: Gráfico comparativo dos gradientes calculados via método adjunto nos códigos Semtex (verde) e FreeFem ++ (laranja) para diferentes valores de $R e$ com velocidade na entrada $U_{\infty}=2,0 \mathrm{~m} / \mathrm{s}$

\subsubsection{Escoamento Através de Um Canal Bidimensional com Variação de Espessura}

\subsubsection{Descrição do Problema}

O segundo caso de estudo corresponde a um escoamento através de um canal bidimensional com variação de espessura. Uma ilustração do problema é exibida a seguir:

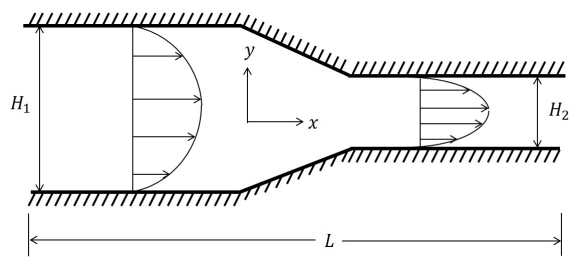

Figura 5.17: Esquema do escoamento através de um canal bidimensional, de comprimento $L$, com variação de espessura. Na primeira parte as placas paralelas e infinitas distam $H_{1}$ uma da outra ao passo que na segunda parte após a restrição - elas distam $H_{2}$ uma da outra sendo $H_{1}>H_{2}$

\subsubsection{Cálculo do Gradiente via Método Adjunto}

Para estre problema, calcular-se-á novamente o gradiente do coeficiente de arrasto em relação à velocidade na fronteira de entrada $U_{\infty}$. A expressão para o cálculo do gradiente via método adjunto é idêntica à expressão utilizada para o 
canal bidimensional sem variação de espessura. Recuperando a expressão:

$$
\frac{\mathrm{d} C_{d}}{\mathrm{~d} U_{\infty}}=\int_{\tilde{S}_{i}} \mathbf{n} \cdot\left\{-\frac{1}{R e}\left[\nabla \varphi+(\nabla \varphi)^{T}\right]+\Theta \mathbb{I}\right\} \frac{\partial \tilde{f}}{\partial U_{\infty}} d \tilde{S}
$$

Nesse problema não é possível encontrar uma expressão analítica de maneira trivial para o cálculo do gradiente. Assim, o gradiente adjunto será comparado apenas com o mesmo calculado via diferenças finitas centradas.

\subsubsection{Soluções do Escoamento e Adjunto - SEMTEX}

Aqui serão apresentadas as soluções das equações do escoamento e adjuntas utilizando o código SEMTEX. A malha utilizada para a simulação das soluções do escoamento e adjunta está exibida a seguir. É uma malha elaborada no software comercial Gambit e possui 3960 elementos quadrilaterais. O teste de convergência para obtenção dessa malha está disponível no apêndice C.

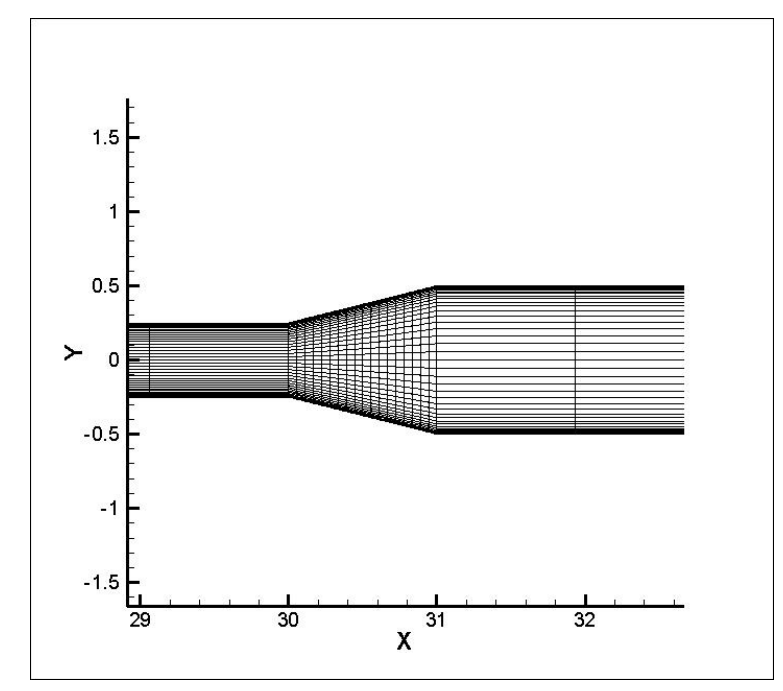

Figura 5.18: Malha Computacional utilizada nas simulações para o escoamento e adjunto

A malha está definida em $-2.5 \leq x \leq 60$ e possui espessura igual a 0,5 unidades na entrada e 1,0 unidade na saída. O trecho no qual ocorre a mudança de espessura do canal está compreendido no intervalo $30 \leq x \leq 31$. Nesta malha, assim como na malha do canal com espessura constante, foi utilizado o artifício de implementar condição de contorno de simetria no trecho que antecede a entrada do canal $(-2.5 \leq x \leq 0)$ para evitar singularidades numéricas. Adiante, na figura 5.19 estão as soluções do escoamento e adjunta para $R e=30$, grau de polinômio $P=10$ e 10000 passos no tempo com magnitude $\Delta t=10^{-3}$ cada um.

À esquerda, solução do escoamento com linhas de corrente, e ao fundo a 


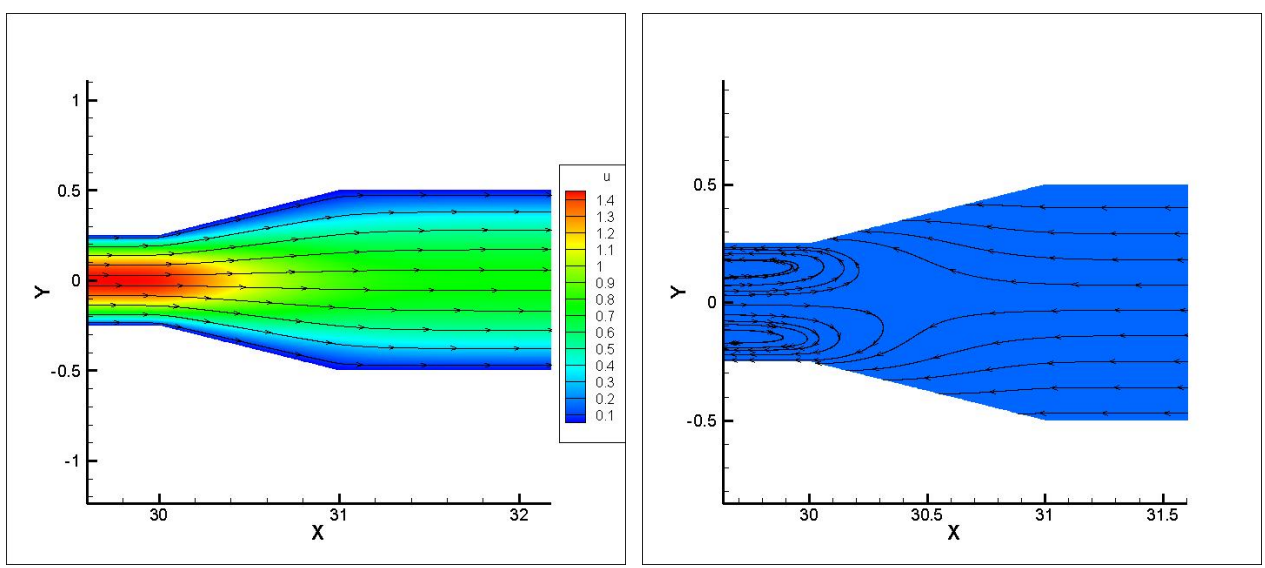

Figura 5.19: Escoamento através de um canal bidimensional com variação de espessura - Detalhes das soluções para o escoamento 2-D e adjunta, $R e=30$, grau de polinômio $\mathrm{P}=10$.

escala de cores corresponde à intensidade da velocidades horizontal $u$. À direita, a solução adjunta com linhas de corrente de velocidade adjunta $\varphi_{k}$, e ao fundo a escala de cores corresponde à intensidade da pressão adjunta $\Theta$.

\subsubsection{Cálculo e Comparativo de Gradientes - SEMTEX}

$\underline{\text { Velocidade na entrada } U_{\infty}=1,0 \mathrm{~m} / \mathrm{s}}$

A seguir, na figura (5.20) estão exibidos os gradientes calculados para diferentes valores de $R e$ e com velocidade do escoamento na entrada $U_{\infty}=1,0 \mathrm{~m} / \mathrm{s}$ via método adjunto e via diferenças finitas centradas, para o código SEMTEX. Neste caso, será feita somente a comparação com o gradiente calculado via diferenças finitas, visto que a obtenção de uma expressão analítica não é trivial. 


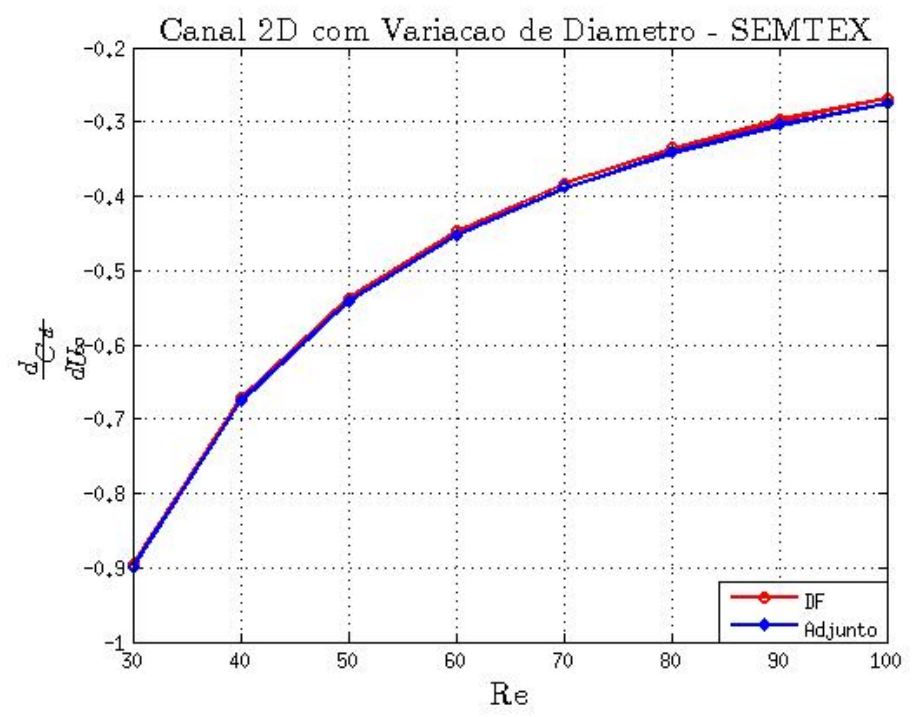

Figura 5.20: Gráfico comparativo dos gradientes calculados via método adjunto (amarelo) e via diferenças finitas (preto) para diferentes valores de $R e$

Neste caso nota-se a proximidade entre os valores dos gradientes calculados pelos dois métodos (erro inferior a $2,5 \%$ )".

\subsubsection{Soluções do Escoamento e Adjunto - FreeFem ++}

Aqui serão apresentadas as soluções das equações do escoamento e adjuntas para o FreeFem ++ . A malha utilizada para a simulação das soluções do escoamento e adjunta está exibida a seguir. Essa malha possui 43594 elementos triangulares. O teste de convergência para obtenção dessa malha também está disponível no apêndice $\mathrm{C}$.

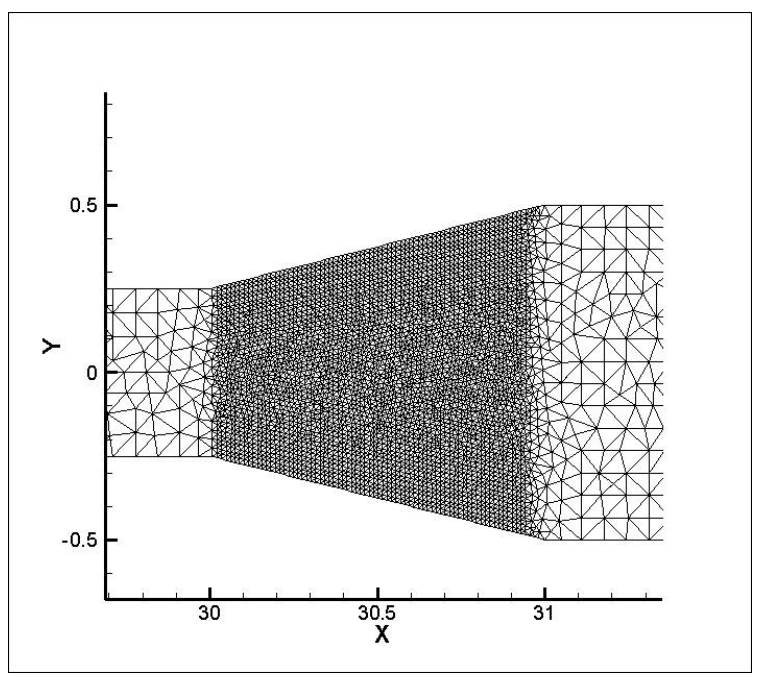

Figura 5.21: Malha Computacional utilizada nas simulações para o escoamento e adjunto 
A malha utilizada neste caso está definida em $-2.5 \leq x \leq 60$ e possui espessura igual a 0,5 unidades na entrada e 1,0 unidade na saída. O trecho no qual ocorre a mudança de espessura do canal está compreendido no intervalo $30 \leq x \leq 31$. Para melhor visualização, foi mostrado apenas o trecho da malha no qual ocorre a mudança de espessura. Na figura (5.22) estão as soluções do escoamento e adjunta para $R e=30$, grau de polinômio $P=4$.
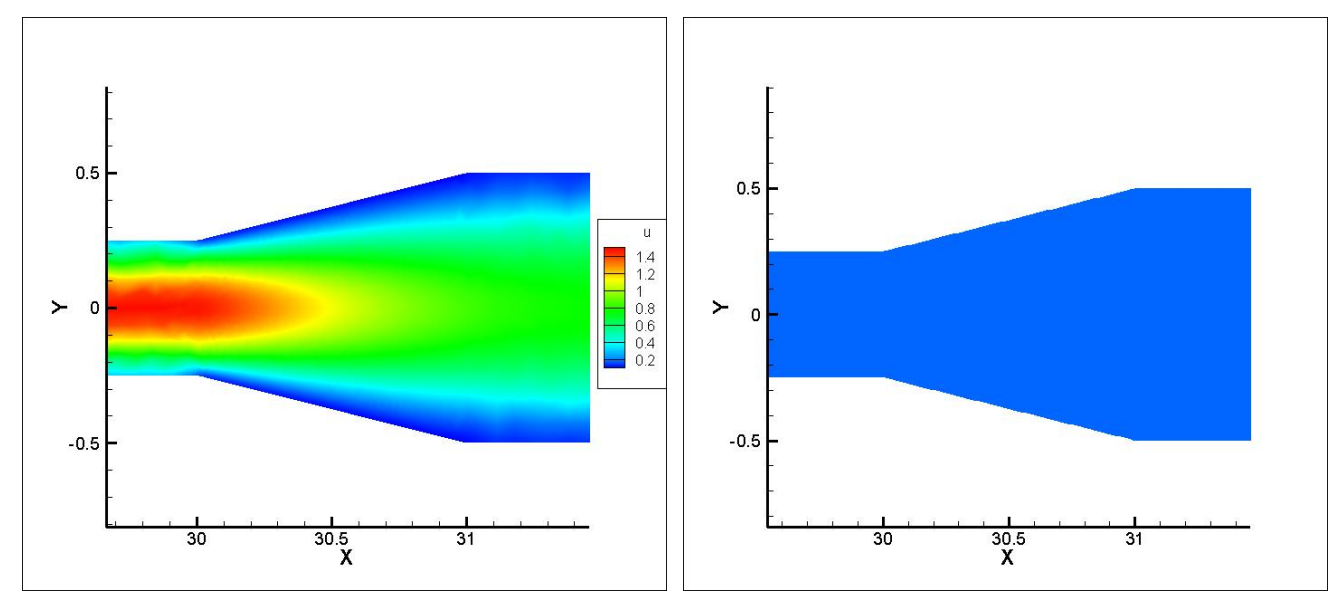

Figura 5.22: Escoamento através de um canal bidimensional com variação de espessura - Detalhes das soluções para o escoamento $2-\mathrm{D}$ e adjunta, $R e=30$, grau de polinômio $\mathrm{P}=4$.

À esquerda, solução do escoamento com escala de cores ao fundo correspondente à intensidade da velocidades horizontal $u$. À direita, a solução adjunta com escala de cores ao fundo correspondente à intensidade da pressão adjunta $\Theta$.

\subsubsection{Cálculo e Comparativo de Gradientes - FreeFem ++}


Para o FreeFem ++ , os resultados estão exibidos na figura (5.23) abaixo: Aqui

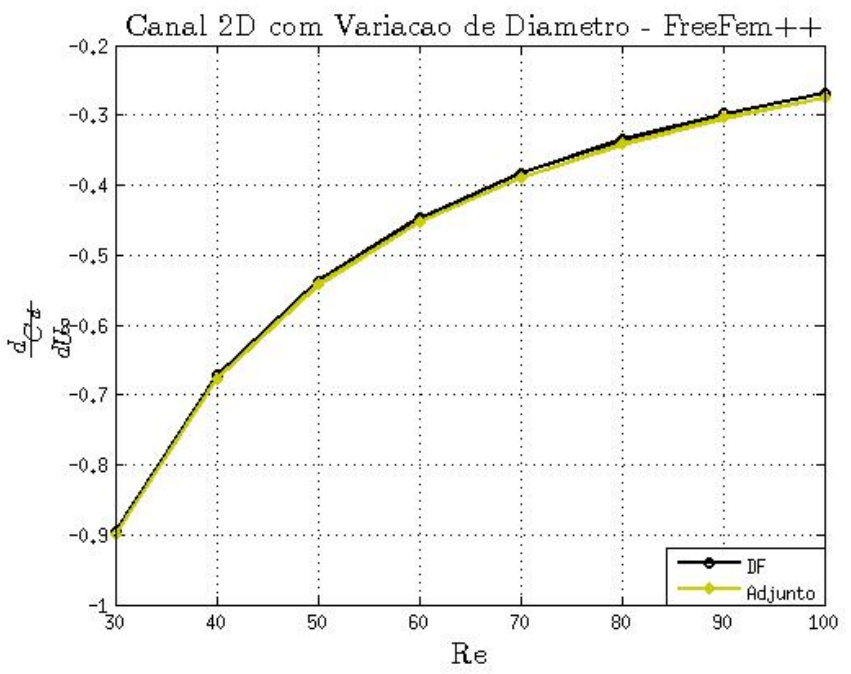

Figura 5.23: Gráfico comparativo dos gradientes calculados via método adjunto (amarelo) e via diferenças finitas (preto) para diferentes valores de $R e$

a proximidade entre os valores também é observada com precisão próxima àquela obtida no SEMTEX (erro inferior a 2,5\%).

\subsubsection{Comparativo de Gradientes - SEMTEX x FreeFem ++}

Comparando-se os gradientes para este caso calculados via SEMTEX e FreeFem ++ :

$\underline{\text { Velocidade na entrada } U_{\infty}=1,0 \mathrm{~m} / \mathrm{s}}$

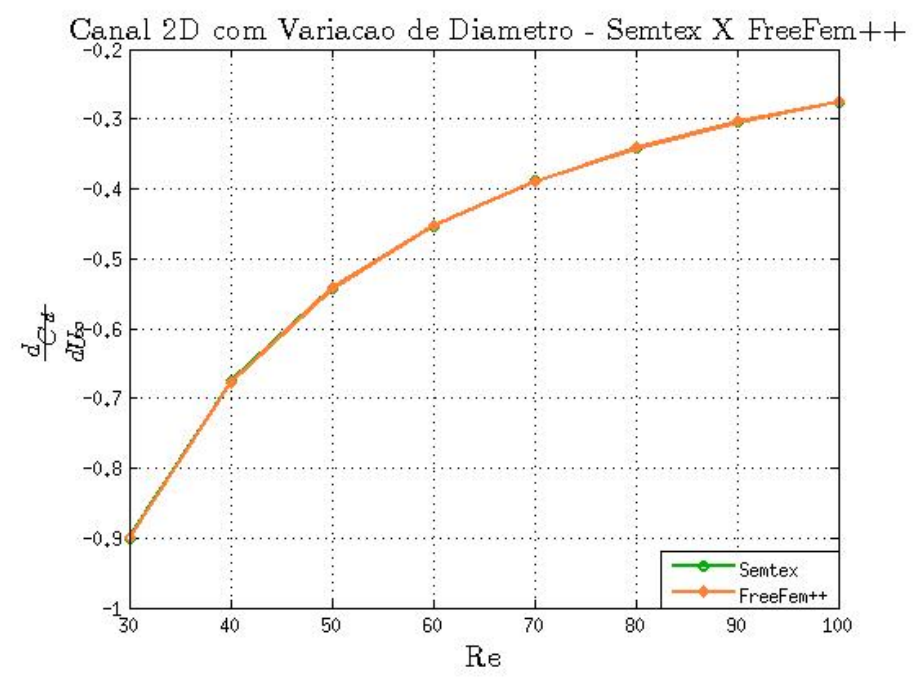

Figura 5.24: Gráfico comparativo dos gradientes calculados via método adjunto nos códigos Semtex (verde) e FreeFem ++ (laranja) para diferentes valores de $R e$ 
Assim como no problema anterior (canal com espessura constante) os valores calculados pelos dois códigos foram próximos (erro inferior a $0,5 \%$ ).

\subsection{Cálculo de sensibilidades geométricas}

Nesta seção será validada a fórmula obtida no capítulo 3 e exibida também neste capítulo (eq. (5.10)) para o gradiente de sensibilidade em relação a parâmetros geométricos, apresentada pela primeira vez na eq. (5.25).

\subsubsection{Escoamento Através de um Canal Bidimensional com Restrição de Espessura}

Para este teste, será estudado o escoamento através de um canal bidimensional com uma restrição de espessura. O esquema do problema a ser estudado está exibido na figura abaixo:

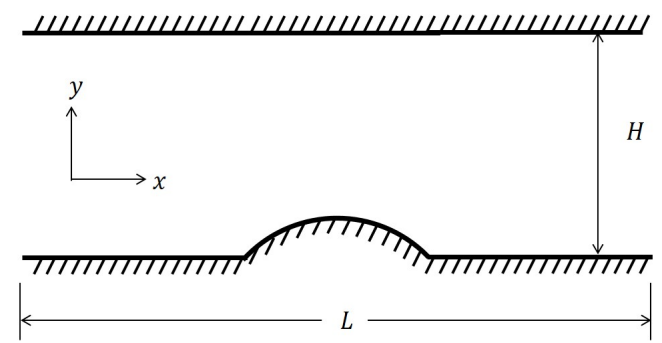

Figura 5.25: Esquema do escoamento através de um canal bidimensional, de comprimento $L$, com restrição de espessura na metade do canal.

\subsubsection{Parametrização da geometria}

Para descrever matematicamente a geometria da restrição, foi escolhida uma parametrização que: permite o cálculo da sensibilidade da medida de mérito em relação a parâmetros da geometria; apresenta unicidade na representação da geometria em questão e possui independência entre os parâmetros. Essa parametrização recebe o nome de parametrização CST (Class-Shape-function Transformation) e sua descrição detalhada está exibida em (KULFAN; BUSSOLETTI, 2006). A seguir é apresentada a formulação básica que foi utilizada nos testes deste trabalho baseando-se na metodologia utilizada em (CHIEREGATTI, 2008) e (CEZE; HAYASHI; VOLPE, 2009).

A equação fundamental da parametrização fornece o valor da ordenada dos 
pontos da geometria:

$$
\tilde{y}(\tilde{x})=C(\tilde{x}) \cdot B(\tilde{x})
$$

onde $\tilde{x}=x / L$ e $\tilde{y}=y / L$ são as coordenadas adimensionais sendo $L$ o comprimento da restrição. As funções $C(\tilde{x})$ e $B(\tilde{x})$ são, respectivamente, função de classe e função de forma. A primeira é dada por:

$$
C(\tilde{x})=\tilde{x}^{N_{1}}(1-\tilde{x})^{N_{2}}
$$

onde $N_{1}$ e $N_{2}$ são expoentes que definem a geometria (KULFAN; BUSSOLETTI, 2006). Para esse teste temos $N_{1}=N_{2}=1$. A função de forma corresponde aos polinômios de Bernstein, uma família de polinômios representada por:

$$
B(\tilde{x})=\sum_{j=0}^{n} a_{k_{j}}\left(\begin{array}{l}
n \\
j
\end{array}\right)(1-\tilde{x})^{n-j} \tilde{x}^{j}
$$

que corresponde ao polinômio de grau $n$ com coeficientes $a_{k}$. Esses coeficientes serão os parâmetros de controle e em relação a eles é que serão calculados os gradientes de sensibilidade. Pensando em um projeto de otimização geométrica (fora do escopo deste trabalho), o cálculo dos gradientes em relação a esses parâmetros, devidamente inseridos em um loop de otimização com as demais etapas (algoritmo de busca, atualização de parâmetros, etc), torna possível a obtenção de novas geometrias atualizando os valores dos coeficientes dos polinômios que compõem a função de forma.

Para fins de validação a metodologia seguida será a mesma empregada ao longo deste trabalho para outros cálculos de sensibilidade ou seja, serão calculados os gradientes de sensibilidade em relação a uma determinada medida de mérito em relação a esses parâmetros utilizando o método adjunto e o método de diferenças finitas. Neste último, cada parâmetro será variado separadamente. Todavia é necessário levar em consideração uma característica dessa família de polinômios que consiste na influência de um coeficiente no outro (CEZE; HAYASHI; VOLPE, 2009). Na referência citada comenta-se que a variação de um coeficiente tem efeitos nos demais inviabilizando, assim, uma análise pontual de cada coeficiente isoladamente. Os únicos coeficientes que não sofrem influência dos demais parâmetros são os coeficientes para $j=0$ e $j=n$. Assim para fins de validação serão utilizados polinômios de grau 1 com dois ceficientes e calcular-se-ão as sensibilidades em relação a esses dois coeficientes $a_{k_{0}}$ e $a_{k_{1}}$. A geração de geometria utilizando essa parametrização segue as seguintes etapas:

- Definição dos pontos pelos quais a geometria deve passar 
- Cálculo dos coeficientes $a_{k}$ utilizando o Método dos Mínimos Quadrados

- Dados os coeficientes $a_{k}$ encontrados, obtém-se pontos intermediários para a geração da malha utilizando os polinômios de Bernstein

\subsubsection{Cálculo do Gradiente via Diferenças Finitas Centradas}

Neste caso, serão necessárias duas simulações para cada parâmetro $a_{k}$ de modo a calcular o gradiente de $C_{d}$ em relação a esses parâmetros via diferenças finitas centradas. Como a geometria escolhida é parametrizada por dois parâmetros $a_{k}$, serão necessárias quatro simulações computacionais. Aqui será observada uma das vantagens do método adjunto que, como já mencionado, é a possibilidade de calcular o gradiente em relação aos dois parâmetros com apenas duas simulações (escoamento e adjunto). As expressões para o gradiente via diferenças finitas estão exibidas a seguir:

$$
\begin{aligned}
\frac{\mathrm{d} C_{d}}{d a_{k_{0}}} & =\frac{\left.C_{d}\right|_{a_{k_{0}}+\delta a_{k_{0}}}-\left.C_{d}\right|_{a_{k_{0}}-\delta a_{k_{0}}}}{2\left(\delta a_{k_{0}}\right)}+\mathcal{O}\left(\delta a_{k_{0}}\right)^{2} \\
\frac{\mathrm{d} C_{d}}{d a_{k_{1}}} & =\frac{\left.C_{d}\right|_{a_{k_{1}}+\delta a_{k_{1}}}-\left.C_{d}\right|_{a_{k_{1}}-\delta a_{k_{1}}}}{2\left(\delta a_{k_{1}}\right)}+\mathcal{O}\left(\delta a_{k_{1}}\right)^{2}
\end{aligned}
$$

\subsubsection{Cálculo do Gradiente via Método Adjunto}

A expressão do gradiente adjunto para este caso está, novamente, exibida a seguir:

$$
\begin{aligned}
T \delta I & =\int_{0} \oint_{B} G \delta\left|\frac{d S^{\prime}}{d S}\right| \mathrm{d} S \mathrm{~d} t+\int_{\Omega}\left(\frac{\Theta}{J}-\varphi_{i} u^{i}\right)\left[\delta\left(J \beta_{q^{\prime}}^{k}\right) u^{q^{\prime}}\right]_{, k} \mathrm{~d} \Omega+ \\
& -\int_{\Omega} \frac{\varphi_{r^{\prime}}}{J}\left\{\partial_{o}\left(\delta J u^{r^{\prime}}\right)+\left[\delta\left(J \beta_{n^{\prime}}^{j}\right)\left(u^{r^{\prime}} u^{n^{\prime}}+g^{r^{\prime} n^{\prime}} p v-\nu u_{, p^{\prime}}^{r^{\prime}} g^{p^{\prime} n^{\prime}}\right)\right]_{, j}\right\} \mathrm{d} \Omega
\end{aligned}
$$

As expressões dos gradientes em relação aos parâmetros $a_{k_{0}}$ e $a_{k_{1}}$ não estão exibidas aqui na forma que foram implementadas nas rotinas de pós processamento para não carregar o texto pois tratam-se de expressões com muitos termos. Inclusive, para chegar às suas respectivas formas finais, utilizou-se o software de manipulação simbólica Mathematica.

Para o cálculo de sensibilidades geométricas, serão testadas duas formas de 
cálculo do gradiente adjunto. Observando a expresão do gradiente acima, nota-se que há duas integrais calculadas ao longo do domínio. Normalmente, integrais assim apresentam maior precisão numérica embora requeiram um custo computacional elevado (KAVVADIAS; KIACHAGIAS; GIANNAKOGLOU, 2015). Uma alternativa a isso é obter uma expressão para o mesmo gradiente que contenha integrais de superfície, reduzindo o custo computacional para o cálculo do gradiente. Esse procedimento é apresentado em (JAMESON; KIM, 2003a) para as equações de Euler. Aqui será testada a expressão do gradiente com integrais de superfície para as equações de Navier-Stokes. A derivação dessas expressões está detalhada no apêndice D pois essa é, também, uma contribuição inédita deste trabalho. Por falta de uma nomenclatura apropriada, o gradiente que envolve integrais de volume será denominado gradiente estendido e o que envolve integrais de superfície, gradiente reduzido. É bom frisar que a nomenclatura adotada não tem relação com a quantidade de termos de cada um deles, foi apenas adotada para diferenciá-los.

Na seção onde serão apresentados os cálculos dos gradientes, serão feitos dois tipos de comparações: comparação do gradiente via diferenças finitas com o gradiente estendido (adjunto) e coparação do gradiente estendido com o reduzido.

\subsubsection{Soluções do Escoamento e Adjunto}

Aqui serão apresentadas as soluções das equações do escoamento e adjuntas utilizando o código SEMTEX. Nesse caso será utilizado somente o SEMTEX para o estudo desse problema. A malha utilizada está exibida abaixo e possui 4800 elementos quadrilaterais. Foi elaborada no software comercial Gambit.

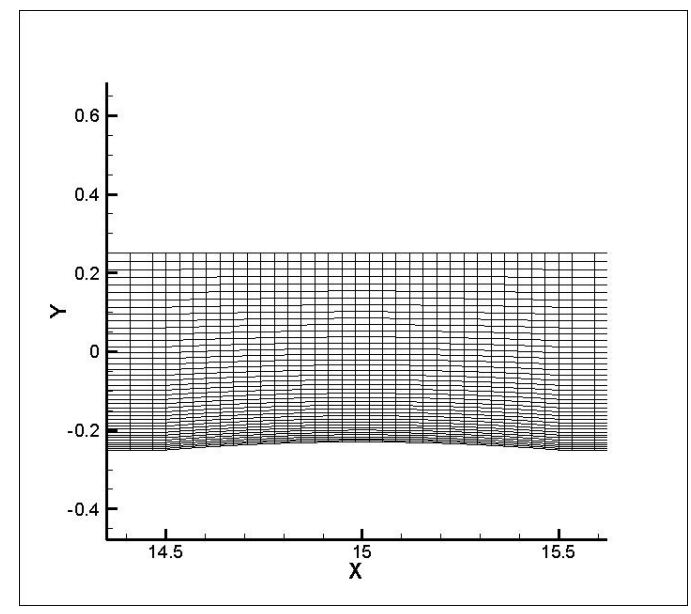

Figura 5.26: Malha Computacional utilizada nas simulações para o escoamento e adjunto 
A malha está definida em $-3 \leq x \leq 30$ com espessura igual a 0,5 e a restrição está contida em $14,5 \leq x \leq 15,5$. Na figura a seguir, estão as soluções do escoamento e adjunta para $R e=30$, grau de polinômio $P=10$ e 10000 passos no tempo com magnitude $\Delta t=10^{-3}$.
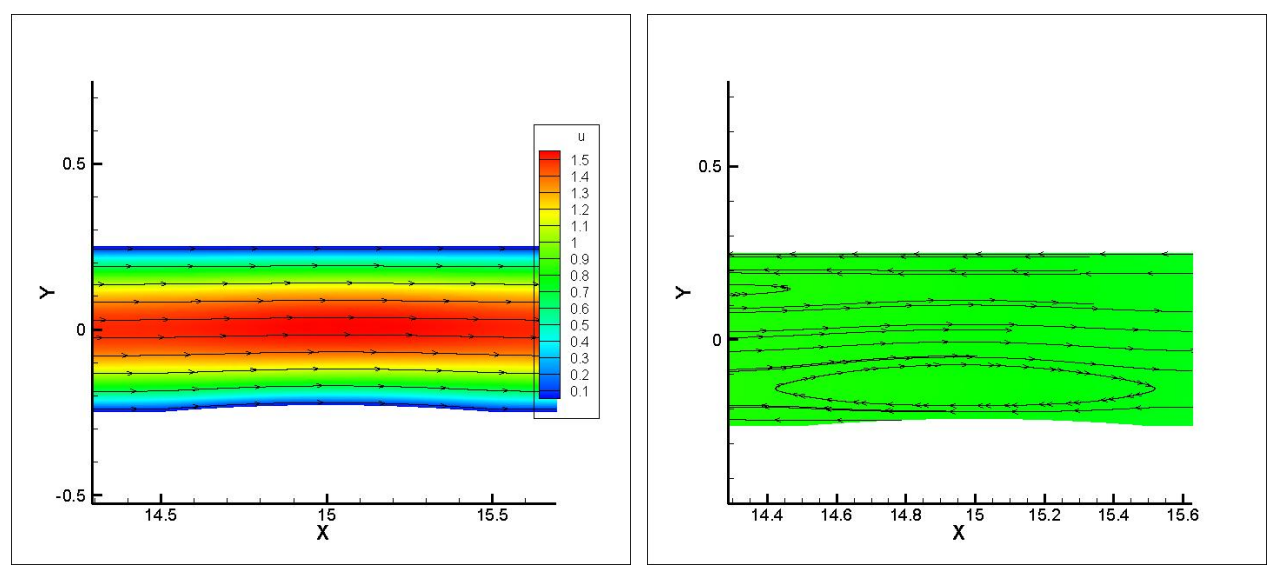

Figura 5.27: Escoamento através de um canal bidimensional com restrição Detalhes das soluções para o escoamento $2-\mathrm{D}$ e adjunta, $R e=30$, grau de polinômio $\mathrm{P}=10$.

À esquerda, solução do escoamento com linhas de corrente, e ao fundo a escala de cores corresponde à intensidade da velocidade horizontal $u$. À direita, a solução adjunta com linhas de corrente de velocidade adjunta $\varphi_{k}$, e ao fundo a escala de cores corresponde à intensidade da pressão adjunta $\Theta$.

\subsubsection{Cálculo e Comparativo de Gradientes}

Gradiente Estendido A seguir, nas figuras (5.28) e (5.29) estão exibidos os gradientes de $F_{x}$ (força exercida pelo fluido nas paredes) em relação aos parâmetros $a_{k_{0}}$ e $a_{k_{1}}$ calculados via método adjunto (formulação estendida) e diferenças finitas centradas. 


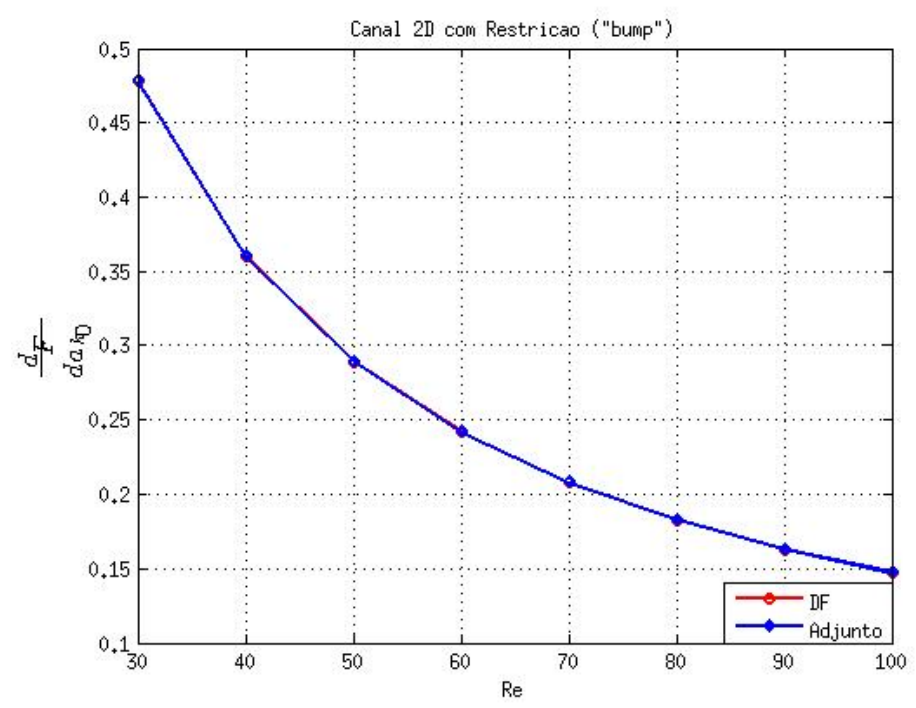

Figura 5.28: Gráfico comparativo dos gradientes calculados via método adjunto (azul) e via diferenças finitas (vermelho) para diferentes valores de $R e$

É possível observar que os gradientes calculados via método adjunto e diferenças finitas estão bastante próximos (erro inferior a 0,08\%). A seguir, os mesmos gradientes calculados em relação ao parâmetro $a_{k_{1}}$ :

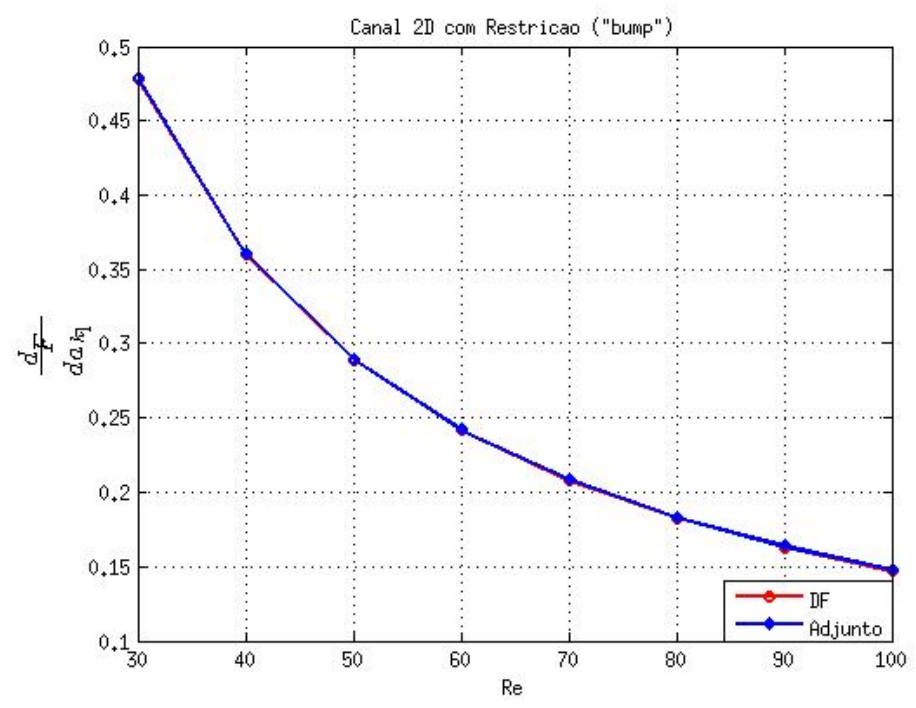

Figura 5.29: Gráfico comparativo do componente $\frac{\partial F_{x}}{\partial a_{k_{0}}}$ do gradiente calculado via método adjunto (azul) e via diferenças finitas (vermelho) para diferentes valores de $R e$ 
Novamente, observa-se a proximidade entre os valores dos gradientes calculados pelos dois métodos (erro inferior a $0,15 \%$ ).

Gradiente Reduzido A seguir, nas figuras (5.30) e (5.31) estão exibidos os gradientes de $F_{x}$ em relação aos parâmetros $a_{k_{0}}$ e $a_{k_{1}}$ calculados via método adjunto (formulação reduzida) e diferenças finitas centradas.

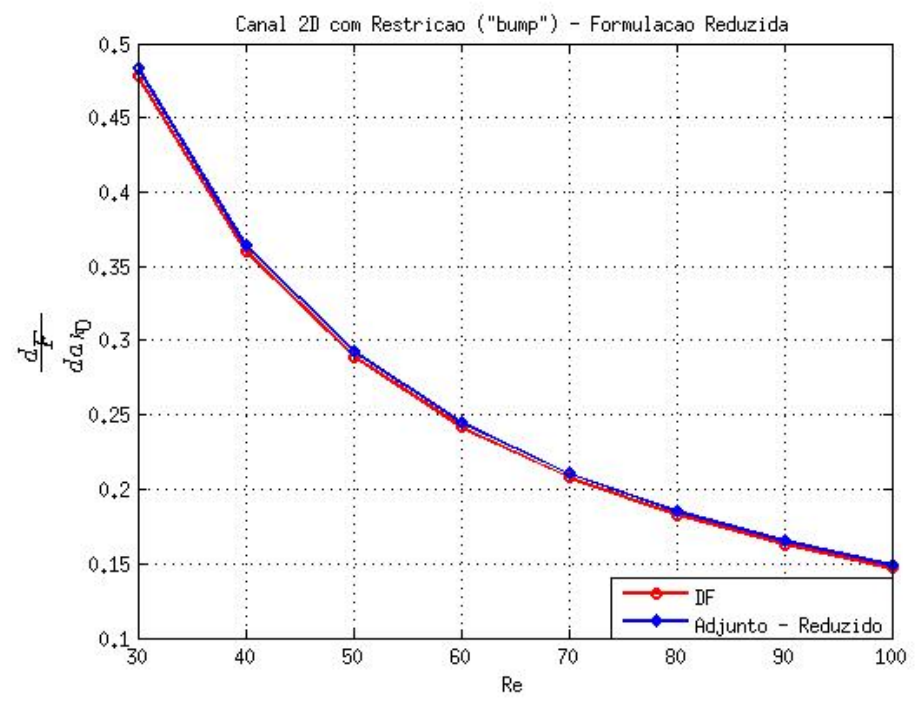

Figura 5.30: Gráfico comparativo do componente $\frac{\partial F_{x}}{\partial a_{k_{1}}}$ do gradiente calculado via método adjunto utilizando a formulação reduzida (azul) e via diferenças finitas (vermelho) para diferentes valores de $R e$ em relação ao parâmetro $a_{k_{0}}$

Observa-se que os valores calculados via método adjunto utilizando a formulação reduzida e vida diferenças finitas estão próximos (erro inferior a 1,2 \%). A precisão nesse caso é inferior à precisão encontrada no comparativo entre a formulação estendida e o método de diferenças finitas pois integrais calculadas em todo o domínio apresentam maior precisão se comparadas com integrais de superfície (KAVVADIAS; KIACHAGIAS; GIANNAKOGLOU, 2015). Entretanto o custo computacional da formulação estendida é maior do que o custo da formulação reduzida visto que o cálculo de integrais de volume requer maior processamento de dados do que o cálculo de integrais de superfície. Uma maneira de melhorar a precisão é utilizar refinamento $p$ ou $h$ na fronteira de integração. Embora encareça o custo computacional ainda será inferior do que a formulação estendida. A seguir, para $a_{k_{1}}$ nota-se que a precisão foi melhor do que para $a_{k_{0}}$ : 


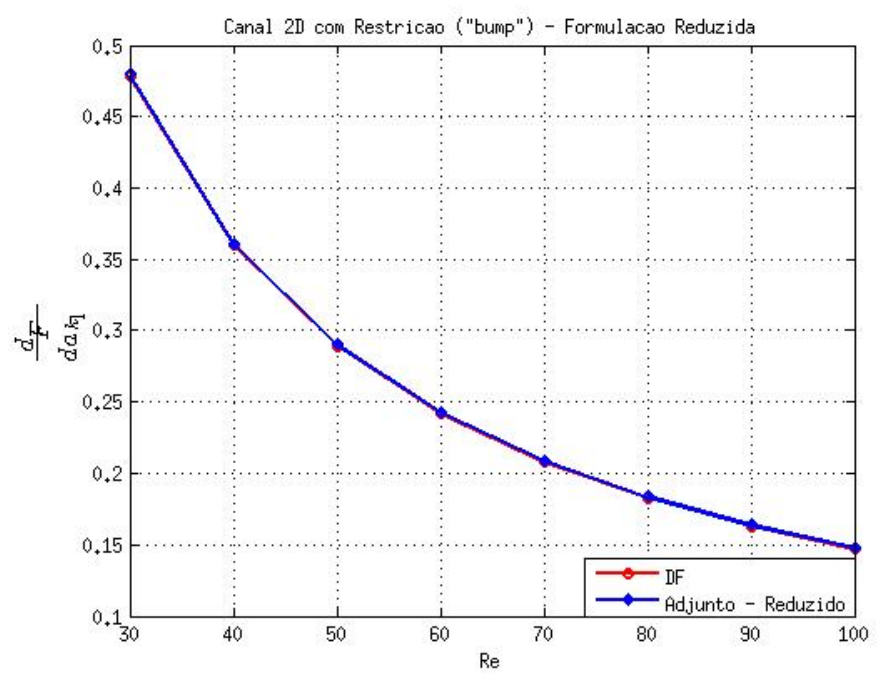

Figura 5.31: Gráfico comparativo dos gradientes calculados via método adjunto utilizando a formulação reduzida (azul) e via diferenças finitas (vermelho) para diferentes valores de $R e$ em relação ao parâmetro $a_{k_{1}}$

Para esse parâmetro nota-se que a precisão é superior (erro inferior a 0,3\%) do que aquela encontrada para o parâmetro $a_{k_{0}}$ (erro inferior a $1,2 \%$ ).

\section{Gradiente Estendido x Gradiente Reduzido}

Nas figuras (5.32) e (5.33) a seguir estão apresentados os gradientes calculados via método adjunto com as duas formulações propostas para fins de comparação e de validação da formulação reduzida.

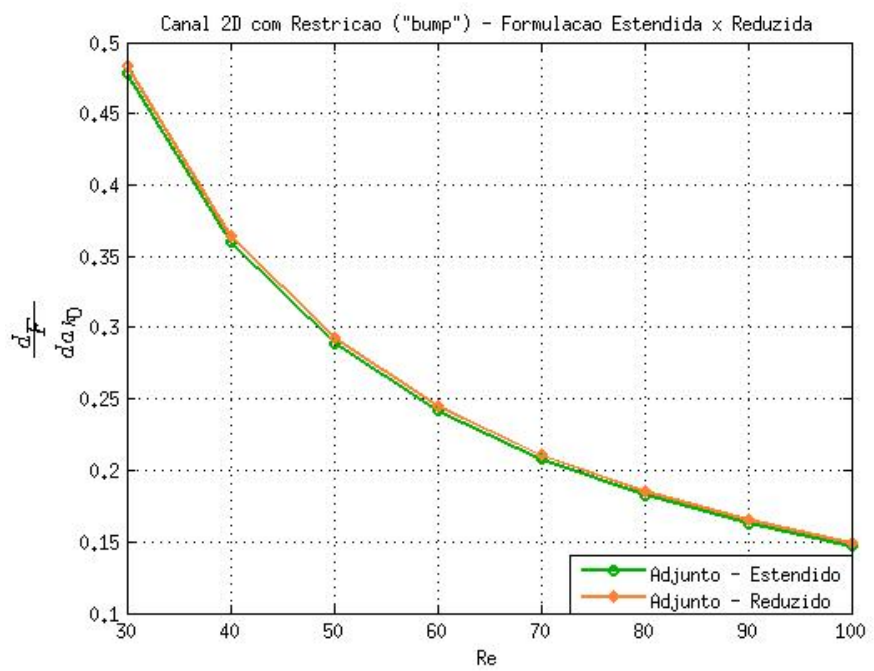

Figura 5.32: Gráfico comparativo dos gradientes calculados via método adjunto utilizando as formulações estendida (verde) e reduzida (laranja) para diferentes valores de $R e$

Observa-se que os valores calculados via método adjunto utilizando as duas 
formulações apresentadas (estendida e reduzida) estão próximos (erro inferior a 1,3\%). A justificativa para essa precisão é a mesma apresentada anteriormente. A seguir, o comparativo para $a_{k_{1}}$ :

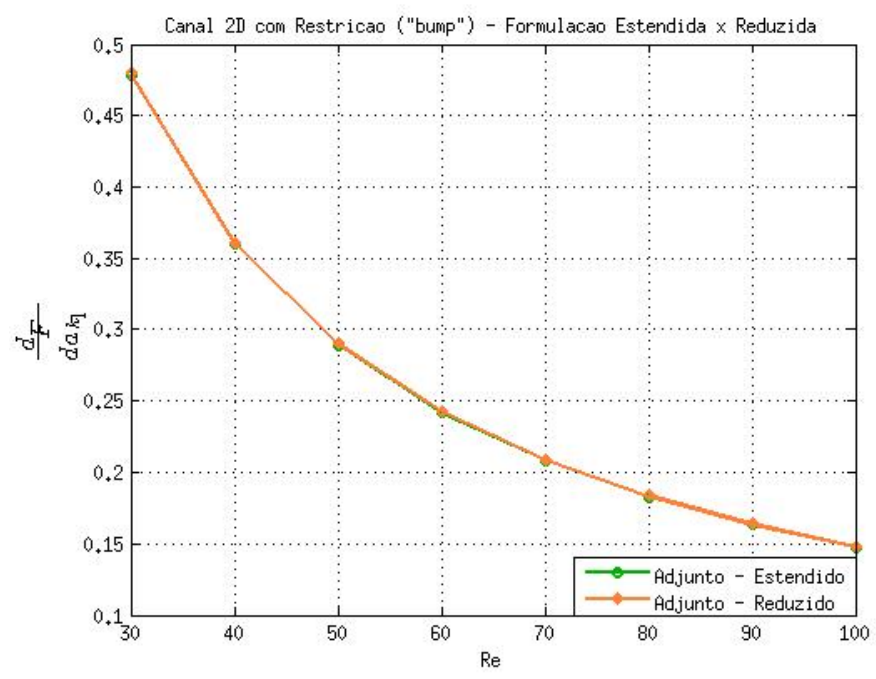

Figura 5.33: Gráfico comparativo dos gradientes calculados via método adjunto utilizando as formulações estendida (verde) e reduzida (laranja) para diferentes valores de $R e$

Para esse parâmetro nota-se, novamente, que a precisão é melhor do que aquela encontrada para o parâmetro $a_{k_{0}}$ (erro inferior a $0,2 \%$ ). 


\section{Conclusões E Trabalhos Futuros}

\subsection{Conclusões}

O objetivo desse trabalho era desenvolver uma metodologia para cálculo de sensibilidades geométricas e não geométricas para escoamentos viscosos incompressíveis utilizando o Método Adjunto. Para tal, uma série de etapas precisaram ser cumpridas para atingir o objetivo proposto inicialmente.

A primeira etapa consistiu em obter as equações adjuntas e o conjunto de condições de contorno para escoamentos viscosos incompressíveis. Para validar esse conjunto de equações obtidos, as equações foram resolvidas nos dois códigos computacionais utilizados no trabalho. Os primeiros testes feitos no SEMTEX validaram as equações encontradas visto que foram obtidas soluções convergidas das equações adjuntas. Como discutido neste trabalho, lançou-se mão da formulação contínua do Método Adjunto embora, na literatura, seja bastante comum o uso da formulação discreta do método. Essa escolha foi tomada para ser possível derivas as expressões dos gradientes de sensibilidade em relação a parâmetros não geométricos, que é a contribuição mais relevante deste trabalho. Além disso há o benefício adicional da independência do método numérico utilizado para resolver as equações adjuntas. Ao obter soluções convergidas das equações adjuntas para o FreeFem + + verificou-se a citada independência do código computacional.

Em seguida, partiu-se para a etapa de validação das expressões encontradas para os gradientes de sensibilidade em relação a parâmetros geométricos e não geométricos, esse último seguindo a abordagem proposta por Cacuci, devidamente citada no capítulo 3. As fórmulas obtidas foram exibidas no capítulo 3, eqs. (5.25) e (3.121). Como o objetivo era validar a nova metodologia proposta, e também por limitações de tempo e escopo, foram escolhidos problemas bidimensionais embora a teoria apresentada seja aplicável a problemas tridimensionais e/ou tempo dependentes sendo necessários apenas mais testes de validação para 
viabilizar tal uso. A validação dos gradientes foi dividida em dois grandes blocos: sensibilidades geométricas e não geométricas. Em todos os testes, as malhas utilizadas foram feitas após um teste de convergência de malhas para as equações do escoamento. Talvez fosse interessante ter feito esse teste também para as equações adjuntas.

No bloco das sensibilidades não geométricas, foram estudados dois problemas distintos e em um deles ainda variou-se a velocidade do escoamento ao longe. A diferença entre os gradientes calculados via método adjunto e via diferenças finitas, em ambos os códigos, não passou de $2.0 \%$ em todos os casos, sendo que em alguns casos chegou a menos de $0.5 \%$. Essa pequena diferença pode ser atribuída a resíduos numéricos das condições de contorno implementadas nos códigos. Possivelmente um melhor trato das malhas em determinadas fronteiras pode reduzir essas pequenas diferenças ainda mais, embora a validação da metodologia proposta tenha sindo atingida.

Já no bloco das sensibilidades geométricas, como foram realizadas duas validações - expressão para o gradiente geométrico e formulação reduzida - optou-se por utilizar somente o SEMTEX em virtude do tempo necessário para as simulações. Aqui, escolheu-se somente um caso para teste no qual os resultados para os gradientes, em função dos parâmetros escolhidos, encontrados via método adjunto foram bastante próximos dos mesmos gradientes encontrados via diferenças finitas. A máxima diferença foi de $0,15 \%$. Além disso, foi possível realizar o mesmo procedimento proposto em (JAMESON; KIM, 2003a) para escoamentos incompressíveis e validá-lo nesta etapa. A diferença entre essa formulação (a qual foi denominada formulação reduzada no cap. 5) e a formulação denominada estendida foi, no máximo, de 1,1 \% em um dos parâmetros. Segundo (KAVVADIAS; KIACHAGIAS; GIANNAKOGLOU, 2015) essa diferença pode ser atribuída ao fato de que integrais de superfície requerem uma malha refinada na região de integração para que a integral apresente boa precisão. Boa precisão essa observada na formulação estendida, a despeito de um custo computacional um pouco mais alto. Aqui ainda foi possível observar uma vantagem do Método Adjunto para o cálculo de sensibilidades: enquanto foram necessárias quatro simulações convergidas para calcular os gradientes via diferenças finitas centradas em relação aos dois parâmetros da geometria, o mesmo cálculo via Método Adjunto requereu apenas duas simulações. Pensando em um problema com muitos parâmetros, o ganho computacional se torna ainda maior.

É importante citar que com alguns casos de validação já rodados foram feitos novos estudos de malhas que indicaram uma melhora do gradiente quando 
determinadas fronteiras (entrada, saída e parede para o caso geométrico) foram refinadas o que sugere que essas imprecisões encontradas tenham natureza numérica. Vale ressaltar que o fato de os valores dos gradientes serem baixos (tanto para sensibilidades geométricas e não-geométricas) e as variações dos parâmetros de geometria $a_{K}$ serem pequenas, contribuem significativamente com o erro. Porém, novamente, acredita-se que com um trabalho diferenciado com as malhas essas imprecisões sejam contornadas.

\subsection{Sugestões para Trabalhos Futuros}

A primeira sugestão para uma continuidade do que foi apresentado neste trabalho é utilizar a expressão do gradiente em relação a parâmetros geométricos e implementar esse cálculo em um ciclo de otimização, como aquele apresentado na seção (1.4). Seria necessário implementar também um algoritmo de busca e de mudança de parâmetros, mas com esses pontos implementados ter-se-á uma ferramenta de otimização para problemas que envolvem escoamentos viscosos e incompressíveis.

Uma segunda sugestão consiste em melhorar o refino das malhas nas fronteiras de modo a reduzir ainda mais as diferenças encontradas entre os gradientes adjunto e via diferenças finitas. Com isso, é possível atacar outra classe de problemas que não foi contemplada aqui, a de escoamentos externos. Como foi identificada a natureza numérica dessas diferenças, com o devido trato nas malhas possivelmente os resultados para escoamentos externos apresentarão a mesma precisão vista aqui para escoamentos internos. 


\section{REFERÊNCIAS}

ALONSO, J. J.; KROO, I. M. Advanced algorithms for design and optimization of quiet supersonic platforms. In: AIAA Computational Fluid Dynamics Conference. Reno, NV: [s.n.], 2002. AIAA-2002-0144.

BARKLEY, D.; BLACKBURN, H. M.; SHERWIN, S. J. Direct optimal growth for timesteppers. International Journal for Numerical Methods in Fluids, v. 1, n. 231, p. 1-21, September 2002.

CACUCI, D. G.; WeBER; F., C.; M., O. E.; MARABLE, J. H. Sensitivity theory for general systems of non-linear equations. Nuclear Science and Engineering, v. 75, p. 88-110, 1980.

CARMO, B. PME 5425- Metodo de Elementos Finitos de Alta Ordem com Aplicacões em Mecânica dos Fluidos e Transferência de Calor. 2014. Departamento de Engenharia Mecânica - EPUSP. Notas de Aula.

CARMO, B. S. Estudo Numerico do Escoamento ao Redor de Cilindros Alinhados. Dissertação (Mestrado) — Universidade de São Paulo, SãoPaulo, SP - Brasil, 2005.

CARRIER, G. F.; PEARSON, C. E. Partial Differential Equations. 2nd. ed. NY: Academic Press, 1988. Theory and Technique.

CEZE, M. A. B.; HAYASHI, M. T.; VOLPE, E. V. A study of the cst parameterization characteristics. In: AIAA. AIAA Applied Aerodynamics Conference. San Antonio, TX, 2009. 2009-3767.

CHIEREGATTI, B. G. Otimizacao aerodinamica de aerofolios utilizando o metodo adjunto. São Paulo, Dezembro 2008.

DUTA, M. C.; GILES, M. B.; CAMPOBASSO, M. S. The harmonic adjoint approach to unsteady turbomachinery design. Int. J. Numer. Meth. Fluids, v. 40, p. $323-332,2002$.

FLüGGE, W. Tensor Analysis and Continuum Mechanics. 1st. ed. N.Y.: Springer-Verlag, 1972.

GELFAND, I. M.; FOMIN, S. V. Calculus of Variations. 1st. ed. N.J.: Prentice-Hall, Inc., 1963. (Selected Russian Publications in Mathematical Sciences). Translated and Edited by R. A. Silverman.

GILES, M.; PIERCE, N.; SüLI, E. Progress in adjoint error correction for integral functionals. Comput Visual Sci, v. 6, p. 113-121, 2004.

GILES, M.; SüLI, E. Adjoint methods for pdes: a posteriori error analysis and postprocessing by duality. Acta Numerica, v. 11, p. 145-236, 2002. 
GILES, M. B. Aerospace Design: a Complex Task. Oxford, July 1997.

HALL, M.; CACUCI, D. Physical interpretation of the adjoint functions for sensitivity analysis of atmospheric models. Journal of Atmospheric Sciences, v. 40, p. 2537-2546, October 1983.

HECHT, F. New development in freefem ++ . Journal of Numerical Mathematics, v. 20, n. 3-4, p. 251-265, 2012.

HIRSCH, C. Numerical Computation of Internal and External Flows. 1st. ed. NY: John Wiley \& Sons, 1994. (Wiley Series in Numerical Methods in Engineering, v. 2). Computational Methods for Inviscid and Viscous Flows.

HIRSCH, C. Numerical Computation of Internal and External Flows. 1st. ed. NY: John Wiley \& Sons, 1994. (Wiley Series in Numerical Methods in Engineering, v. 1). Fundamentals of Numerical Discretization.

JAMESON, A. Aerodynamic design via control theory. In: 12th IMACS World Congress on Scientific Computation. Paris: [s.n.], 1988. (MAE Report 1824).

JAMESON, A. Optimum Aerodynamic Design via Boundary Control. [S.l.], April 1994.

JAMESON, A. Re-engineering the design process through computation. In: AMERICAN INSTITUTE OF AERONAUTICS AND ASTRONAUTICS. 35th Aerospace Sciences Meeting $\&$ Exhibit. Reno, NV: AIAA, 1997. AIAA-97-0641.

JAMESON, A.; KIM, S. Reduction of the adjoint gradient formula for aerodynamic shape optimization problems. AIAA Journal, v. 41, n. 11, p. 2114-2129, November 2003.

JAMESON, A.; KIM, S. Reduction of the adjoint gradient formula in the continuous limit. In: AIAA Computational Fluid Dynamics Conference. [S.l.: s.n.], 2003. AIAA 2003-0040.

JAMESON, A.; MARTINELLI, L.; PIERCE, N. A. Optimum aerodynamic design using the Navier-Stokes equations. Theoretical and Computational Fluid Dynamics, v. 1, n. 10, p. 213-237, 1998.

JAMESON, A.; SRIRAM, A.; MARTINELLI, L. A continuous adjoint method for unstructured grids. In: AIAA Computational Fluid Dynamics Conference. Orlando, FL: [s.n.], 2003. AIAA 2003-3955.

KAPLAN, W. Advanced Calculus. NY: Addison-Wesley Pub. Co., 1971.

KARNIADAKIS, G. E.; SHERWIN, S. Spectral/hp element methods for CFD. 2nd. ed. New York: Oxford University Press, 2005.

KARPOUZAS, G. K.; KIACHAGIAS, E. M. P.; SCHUMACHER, T.; VILLIERS, E.; GIANNAKOGLOU, K. C.; OTHMER, C. Adjoint optimization for vehicle external aerodynamics. In: JSAE Annual Congress. Japan: [s.n.], 2015. p. $1-7$.

KAVVADIAS, I. S.; KIACHAGIAS, E. M. P.; GIANNAKOGLOU, K. C. On the proper treatment of grid sensitivities in continuous adjoint methods for shape optimization. Computers and Fluids, v. 301, p. 1-18, 2015. 
KIACHAGIAS, E. M. P.; GIANNAKOGLOU, K. C. Continuous adjoint methods for turbulent flows, applied to shape and topology optimization: Industrial applications. Archives of Computational Methods in Engineering, -, p.,- 2015.

KIACHAGIAS, E. M. P.; MAGOUlAS, N.; GIANNAKOGLOU, K. C.; OTHMER, C.; MUELLER, J. Noise reduction in car aerodynamics using a surrogate objective function and the continuous adjoint method with wall functions. Computers and Fluids, v. 122, p. 223-232, 2015.

KIM, H.; NAKAHASHI, K. Unstructured adjoint method for Navier-Stokes equations. JSME International Journal, v. 48, n. 2, 2005.

KIM, S.; ALONSO, J. J.; JAMESON, A. Multi-element high-lift configuration design optimization using viscous continuous adjoint method. Journal of Aircraft, v. 41, n. 5, September-October 2004.

KULFAN, B. M.; BUSSOLETTI, J. E. Fundamental parametric geometry representations for aircraft component shapes. In: 11th AIAA Multidisciplinary Analysis and Optimization Conference. Portsmouth: [s.n.], 2006. AIAA-20066948 .

LAX, P. D. Linear Algebra and Its Applications. 2nd. ed. NJ: Wiley, 2007. (Pure and Applied Mathematics).

LIONS, J. L. Optimal Control of Systems Governed by Partial Differential Equations. 1st. ed. Berlin: Springer-Verlag, 1971. (Die Grundelehren der mathematischen Wissenschaften, 170).

LUSTERNICK, L. A.; SOBOLEV, V. J. Elements of Functional Analysis. 1st. ed. Delhi: Hindustan Pub. Co., 1961. (Russian Monographs and Texts on Advanced Mathematics and Physics, V).

MACCORMACK, R. A perspective on a quarter century of cfd research. In: AMERICAN INSTITUTE OF AERONAUTICS AND ASTRONAUTICS. 11th AIAA Computational Fluid Dynamics Conference. Orlando, FL: AIAA, 1993. AIAA-93-3291.

MAO, X. Vortex Instability and Transient Growth. Tese (Doutorado) - Imperial College of Science, Technology and Medicine, London, UK, 2010.

MOHAMADI, B.; PIRRONEAU, O. Applied Shape Optimization for Fluids. 1st. ed. [S.1.]: Oxford University Press, 2001.

MORSE, P. M.; FESHBACH, H. Methods of Theoretical Physics. 1st. ed. NY: MacGraw-Hill, 1953.

MORSE, P. M.; FESHBACH, H. Methods of Theoretical Physics. 1st. ed. NY: MacGraw-Hill, 1953.

NADARAJAH, S.; JAMESON, A. Optimum shape design for unsteady three-dimensional viscous flows using a non-linear frequency domain method. In: AMERICAN INSTITUTE OF AERONAUTICS AND ASTRONAUTICS. 24th Applied Aerodynamics Conference. San Francisco, CA: AIAA, 2007. 
NADARAJAH, S. K.; JAMESON, A. A comparison of the continuous and discrete adjoint approach to automatic aerodynamic optimization. AIAA, 2000.

NADARAJAH, S. K.; JAMESON, A. Optimum shape design for unsteady flows with time-accurate continuous and discrete adjoint methods. AIAA Journal, v. 45 , n. 7 , p. $1478-1491$, July 2007.

PETER, J. E. V.; DWIGHT, R. P. Numerical sensitivity analysis for aerodynamic optimization: A survey of approaches. Computers and Fluids, v. 39, p. 373-391, 2010.

PIRRONEAU, O. On optimal profiles in stokes flow. Journal of Fluid Mechanics, v. 59, n. 1, p. 117-128, 1973.

PIRRONEAU, O. On optimal design in fluid dynamics. Journal of Fluid Mechanics, v. 64, n. 1, p. 97-110, 1974.

PISKUNOV, N. S. Differential and Integral Calculus. 1st. ed. Moscow: Mir Publishers, 1968. Translated from Russian by G. Yankovsy.

REUTHER, J. J. Aerodynamic Shape Optimization Using Control Theory. Tese (Doutorado) - University of California, 1996.

SANTOS, L. C. C. A Hybrid Inverse Optimization Method for Aerodynamic Design of Lifting Surfaces. Tese (Doutorado) - Georgia Institute of Technology, 1993.

SANTOS, L. C. C. A Study on Aerodynamic Design Optimization Using An Adjoint Method. Braunschweig, July 1995.

SPIVAK, M. Calculus on Manifolds - a Modern Approach to Classical theorems of Advanced Calculus. 24th. ed. NY: Addison-Wesley Pub. Co., 1995.

VENDITTI, D. A.; DARMOFAL, D. L. A multilevel error estimation and grid adaptive strategy for improving the accuracy of integral outputs. 1999. AIAA Paper 99-3292.

VENDITTI, D. A.; DARMOFAL, D. L. Adjoint error estimation and grid adaptation for functional outputs: Application to quasi-one-dimensional flow. Journal of Computational Physics, v. 164, p. 204-227, 2000.

VENDITTI, D. A.; DARMOFAL, D. L. Anisotropic grid adaptation for functional outputs: application to two-dimensional viscous flows. Journal of Computational Physics, v. 187, p. 22-46, 2003.

VOLPE, E. V. Estudo da Transferência de Calor e Quantidade de Movimento no Escoamento Laminar através de Passagens com Geometria Arbitrária: Modelos Compressivel e Incompressivel. Dissertação (Mestrado) - Escola Politécnica da Universidade de São Paulo, São Paulo, SP, Brasil, 1993.

VOLPE, E. V. Continuous Formulation of the Adjoint Problem for Unsteady Incompressible Navier-Stokes Flows. SP, June 2011. Research Project:

ANP-CFD-ANFLEX, Desenvolvimento de Modelos Preditivos para Análise do Comportamento Dinâmico de Risers.

WHITE, F. M. Viscous Fluid Flow. 3rd. ed. [S.1.]: McGraw Hill, 2006. 
XU, S.; JAHN, W.; MüLLER, J.-D. Cad-based shape optimisation with cfd using a discrete adjoint. International Journal for Numerical Methods in Fluids, v. 74 , p. $153-168,2013$. 


\section{ApÊNDICE A - Forma GeneralizadA DAS EquaÇÕes DE NAVIER StOKes}

Neste apêndice é apresentado o conjunto de equações de Navier-Stokes escrito em coordenadas generalizadas. É possível encontrar duas maneiras distintas para descrever tais equações desse modo: a forma estendida na qual os operadores diferenciais estão completamente generalizados; e a forma reduzida a qual envolve tanto coordenadas cartesianas como coordenadas transformadas. Para a primeira forma são necessárias noções a respeito do formalismo em álgebra tensorial generalizada (FLüGGE, 1972; VOLPE, 1993). Já para a segunda forma, as equações podem ser obtidas simplesmente pelo uso de um operador de transformação (JAMESON; MARTINELLI; PIERCE, 1998). Neste trabalho faz-se uso de ambas as formas e, por isso, é conveniente mostrar que as duas formas são, de fato, equivalentes.

\section{A.1 Introdução}

Admite-se aqui que o domínio do escoamento é descrito em coordenadas cartesianas enquanto o espaço transformado é descrito em coordenadas generalizadas. Admite-se também que no espaço físico as coordenadas generalizadas conformamse às fronteiras do corpo, para que a especificação das condições de contorno seja a mais simples possível. Um esquema dessa relação entre espaços pode ser visto na figura A.1:

$$
\left\{\begin{array}{l}
\text { Domínio do Escoamento } \Rightarrow\left(x^{j^{\prime}}, t\right) \\
\text { Espaço Transformado } \Rightarrow\left(\xi^{i}, t\right)
\end{array}\right.
$$

Para ser possível diferenciar ambos os sistemas de coordenadas, todos os elementos que estiverem sendo expressos em coordenadas cartesianas serão identificados com um apóstrofo $\left({ }^{\prime}\right)$. Elementos que não estiverem idenficados com o apóstrofo serão elementos no espaço transformado. Definindo-se os operadores de transfor- 


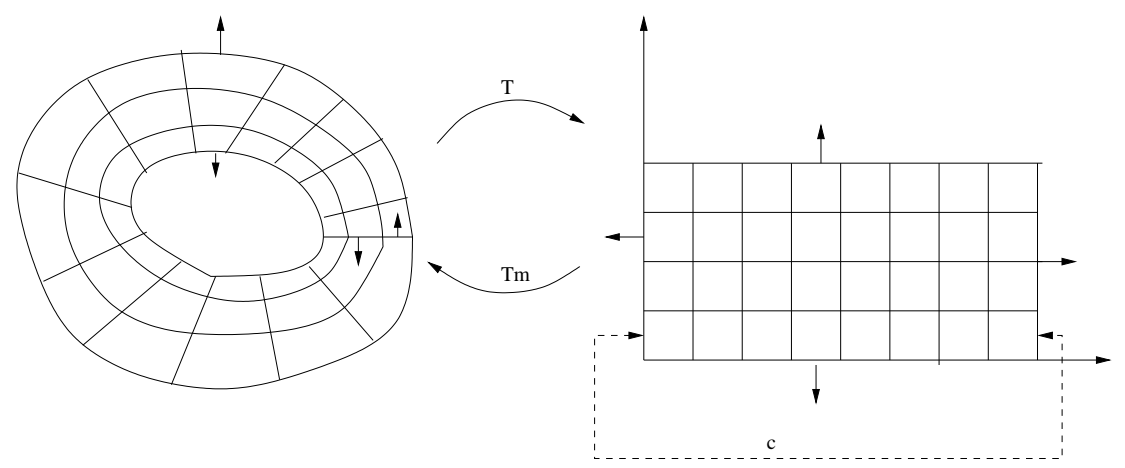

Figura A.1: Espaços físico e transformado e a relação entre eles por meio do operador $\beta$ e seu inverso $\beta^{-1}$

mação:

$$
\left\{\begin{array}{cll}
\beta \Rightarrow \beta_{j^{\prime}}^{i}=\frac{\partial \xi^{i}}{\partial x^{j^{\prime}}} \quad: \quad u^{i}=\beta_{j^{\prime}}^{i} u^{j^{\prime}} \\
\beta^{-1} \Rightarrow \beta_{i}^{j^{\prime}}=\frac{\partial x^{j^{\prime}}}{\partial \xi^{i}} \quad: \quad u^{j^{\prime}}=\beta_{i}^{j^{\prime}} u^{i}
\end{array}\right.
$$

O Jacobiano da transformação é dado por: $J=\operatorname{det}\left(\beta_{i}^{j^{\prime}}\right)$. Aqui, entendese que as componentes contravariante $\left(u^{i}\right)$ e covariante $\left(u_{i}\right)$ do vetor são iguais somente para o sistema cartesiano. O conjunto das equações de Navier-Stokes para escoamentos viscosos e incompressíveis pode ser completamente expresso por (HIRSCH, 1994b):

$$
\begin{cases}\nabla \cdot(\mathbf{u}) & =0 \\ \frac{\partial(\mathbf{u})}{\partial t}+\nabla \cdot(\mathbf{u u}) & =-\frac{\nabla p}{\rho}+\frac{1}{\rho} \nabla \cdot \tau\end{cases}
$$

O tensor das tensões viscosas $\tau$, é expresso por:

$$
\boldsymbol{\tau}=\mu\left[\left(\nabla \mathbf{u}+\nabla \mathbf{u}^{T}\right)-\frac{2}{3} \mathbf{I} \nabla \cdot \mathbf{u}\right]
$$

onde o símbolo I denota o tensor identidade. Neste apêndice, as propriedades $\mu$ e $k$ são assumidos como constantes. A versão generalizada e completa da equação (A.3) é obtida escrevendo todos os operadores diferenciais na forma generalizada (FLüGGE, 1972). Tal procedimento leva a:

$$
\left\{\begin{array}{l}
\partial_{o} \rho+\left.\left(\rho u^{k}\right)\right|_{k}=0 \\
\partial_{o}\left(\rho u^{i}\right)+\left.\left(\rho u^{i} u^{j}\right)\right|_{j}=-\left.\left(g^{i j} p\right)\right|_{j}+\left.\tau^{i j}\right|_{j}
\end{array}\right.
$$

Aqui, o símbolo $\partial_{o}$ denota diferenciação temporal, $\partial_{o}=\partial / \partial t$. Seguindo a mesma notação, o tensor das tensões generalizado é expresso por:

$$
\tau^{i j}=\mu\left[\left(\left.u^{i}\right|^{j}+\left.u^{j}\right|^{i}\right)-\left.\frac{2}{3} g^{i j} u^{k}\right|_{k}\right]
$$

Em princípio, há duas maneiras distintas de derivar o conjunto de equações 
descrito acima. Na primeira, aplica-se o operador de transformação (A.2) diretamente às equações de Navier-Stokes em coordenadas cartesianas ou; ou pode-se partir da forma generalizada completa (A.5) e obter a forma reduzida após manipulações algébricas. Aqui, obter-se-ão as equações reduzidas através da segunda maneira descrita. Depois, será mostrada a equivalência entre ambas as metodologias.

\section{A.2 Noções de Álgebra Tensorial Generalizada}

Aqui serão apresentadas passagens, noções e conceitos que são utilizados na derivação de equações o capítulo (3). Alguns conhecimentos prévios de álgebra tensorial serão utilizados.

Partir-se-á dos operadores diferenciais que são exibidos em (A.5) e (A.6). A derivada covariante define uma versão generalizada do operador gradiente $(\nabla)$ (FLüGGE, 1972). A expressão depende da variância da entidade sobre a qual ele opera. Em se tratando de escalares redundará em derivadas parciais do tipo $\left.p\right|_{k}=p_{, k}$, onde a vírgula indica a diferenciação parcial ()$_{, k}=\partial / \partial \xi^{k}$. Já para vetores, ocorre o seguinte:

$$
\left\{\begin{array}{l}
\left.u^{i}\right|_{j}=u_{, j}^{i}+u^{k} \Gamma_{j k}^{i} \\
\left.u_{i}\right|_{j}=u_{i, j}-u_{k} \Gamma_{i j}^{k}
\end{array}\right.
$$

Como resultado, são obtidos tensores de segunda ordem, cuja variância pode ser modificada através do tensor métrico $g^{i j}:\left.u_{i}\right|_{j} g^{i k} g^{j p}=\left.u^{k}\right|^{p}$. Para tensores de segunda ordem, têm-se (FLüGGE, 1972):

$$
\left\{\begin{array}{l}
\left.A_{i j}\right|_{k}=A_{i j, k}-A_{l j} \Gamma_{i k}^{l}-A_{i l} \Gamma_{k j}^{l} \\
\left.A_{j}^{i}\right|_{k}=A_{j, k}^{i}+A_{j}^{l} \Gamma_{k l}^{i}-A_{l}^{i} \Gamma_{j k}^{l} \\
\left.A_{i}^{j}\right|_{k}=A_{i, k}^{j}-A_{l}^{j} \Gamma_{i k}^{l}+A_{i}^{l} \Gamma_{k l}^{j} \\
\left.A^{i j}\right|_{k}=A_{, k}^{i j}+A^{l j} \Gamma_{k l}^{i}+A^{i l} \Gamma_{k l}^{j}
\end{array}\right.
$$

Como resultados, obtém-se tensores de terceira ordem, que também podem ter sua variância modificada através do tensor métrico.

É conveniente mencionar duas propriedades relevantes da derivada covariante. A primeira é que ela satisfaz a regra de diferenciação do produto de tensores. A segunda, diz que a ordem na segunda derivada covariante pode ser modificada caso o vetor de espaço não apresente nenhuma curvatura intrínseca, embora as 
coordenada, por si só, possam ser curvilíneas. (FLüGGE, 1972).

$$
\begin{aligned}
\left.\left(u_{i} v_{j}\right)\right|_{k} & =\left.u_{i}\right|_{k} v_{j}+\left.u_{i} v_{j}\right|_{k} \\
\left.\left(u_{i} A^{i j}\right)\right|_{j} & =\left.u_{i}\right|_{j} A^{i j}+\left.u_{i} A^{i j}\right|_{j} \\
\left.\left(u^{i} A_{i j}\right)\right|^{j} & =u^{i}{ }^{j} A_{i j}+\left.u^{i} A_{i j}\right|^{j} \\
\left.\left.u_{i}\right|_{j}\right|_{k} & =\left.\left.u_{i}\right|_{k}\right|_{j} \text { em espaços que não apresentam curvatura intrínseca }
\end{aligned}
$$

Em todo este trabalho, o vetor de espaço, $\mathbf{R}^{3}$, que não apresenta nenhuma curvatura intrínseca e pode ser descrito tanto em coordenadas cartesianas quanto curvilíneas.

O símbolo $\Gamma_{j k}^{i}$, exibido nas equações acima, denotam os símbolods de Christoffel. Em resumo, eles levam em consideração os efeitos de curvatura locais no sistema de coordenadas, na diferenciação (FLüGGE, 1972). Duas propriedades de $\Gamma_{j k}^{i}$ são importantes para as deduções apresentadas neste trabalho: os símbolos de Christoffel são simétricos em relação aos seus dois primeiros índices; e o terceiro índice, isoladamente, pode subir ou descer por meio do tensor métrico.

$$
\left\{\begin{array}{l}
\Gamma_{i j}^{k}=\Gamma_{j i}^{k} \\
\Gamma_{i j}^{k} g_{k n}=\Gamma_{i j n}
\end{array}\right.
$$

É importante ressaltar que esses entes não são tensores de terceira ordem. Uma maneira de calcular os símbolos de Christoffel em termos dos operadores de transformação será derivada a seguir:

Os símbolos $g_{i j}$ e $g^{i j}$ correspondem às componentes covariante e contravariante do tensor métrico, respectivamente: $(\mathrm{d} s)^{2}=g^{i j} \mathrm{~d} \xi_{i} \mathrm{~d} \xi_{j}=g_{i j} \mathrm{~d} \xi^{i} \mathrm{~d} \xi^{j}$. Algumas propriedades relevantes dessas componentes: são simétricas e assumem forma diagonal se associadas com sistemas de coordenadas localmente ortogonais.

Em todos os casos aqui abordados, o sistema de coordenadas é assumido como sendo localmente ortogonal. Com isso, os termos fora da diagonal do tensor métrico são sempre nulos. Uma identidade útil, e geral, que permite inverter o tensor métrico $g^{i j} g_{j k}=\delta_{k}^{i}$, é mostrada a seguir:

$$
\begin{aligned}
g^{i k} & =\delta_{j}^{i} g^{j k}=\frac{1}{2} \epsilon^{i p q} \epsilon_{j p q} g^{j k} \\
& =\frac{1}{2} \epsilon^{i p q} \epsilon^{r l m} g_{r j} g_{p l} g_{q m} g^{j k} \\
& =\frac{1}{2} \epsilon^{i p q} \epsilon^{k l m} g_{p l} g_{q m}
\end{aligned}
$$

sendo $\epsilon^{i j k}$ o tensor de permutação e $\delta_{j}^{i}$, o delta de Kronecker. 
Os tensores de permutação, na forma que eles aparecem na equação (A.10), são associados com o espaço transformado. Eles são definidos em termos dos símbolos de permutação tais como $\epsilon_{i j k}=J e_{i j k}$, para componente covariantes; e tais como $\epsilon^{i j k}=J^{-1} e^{i j k}$, para componente contravariantes. Tais símbolos são conhecidos como:

$e^{i j k}=e_{i j k}=\left\{\begin{array}{l}+1 \text { se }(i, j, k)=(1,2,3) \text { ou qualquer permutação par nessa sequência } \\ -1 \text { se }(i, j, k) \text { qualquer permutação ímpar dos índices acima } \\ 0 \quad \text { se quaisquer dois subescritos forem iguais }\end{array}\right.$

Diferentemente de tensores, os símbolos de permutação não estão associados com nenhum sistema de coordenadas. Além disso, somente no sistema cartesiano tensores e símbolos de permutação são coincidentes.

De maneira similar à equação (A.10), uma expressão para o operador da transformada inversa, $\beta_{j^{\prime}}^{i} \beta_{k}^{j^{\prime}}=\delta_{k}^{i}$, é obtido fazendo:

$$
\begin{aligned}
\delta_{n}^{k} & =\frac{1}{2} \epsilon^{i j k} \epsilon_{i j n}=\frac{1}{2} \epsilon^{i j k} \epsilon_{r^{\prime} l^{\prime} s^{\prime}} \beta_{i}^{r^{\prime}} \beta_{j}^{l^{\prime}} \beta_{n}^{s^{\prime}} \\
\beta_{p^{\prime}}^{k} \beta_{n}^{p^{\prime}} & =\frac{1}{2 J} e^{i j k} e_{r^{\prime} l^{\prime} s^{\prime}} \beta_{i}^{r^{\prime}} \beta_{j}^{l^{\prime}} \beta_{n}^{s^{\prime}} \\
\beta_{q^{\prime}}^{k} & =\frac{1}{2 J} e^{i j k} e_{r^{\prime} l^{\prime} q^{\prime}} \beta_{i}^{r^{\prime}} \beta_{j}^{l^{\prime}}
\end{aligned}
$$

onde se fez uso da relação entre tensores e símbolos de permutação, bem como a igualdade entre ambos os entes no sistema cartesiano (FLüGGE, 1972; VOLPE, 1993). A última linha da equação acima é obtida multiplicando a anterior por $\beta_{q^{\prime}}^{n}$

Ao multiplicar a última linha de (A.12) por $J$, substituindo $\left(\beta_{i}^{r^{\prime}} \beta_{j}^{l^{\prime}}\right)$ por suas definições, (A.2), e diferenciando o resultado, chega-se a:

$$
\begin{aligned}
\frac{\partial}{\partial \xi^{k}}\left(J \beta_{q^{\prime}}^{k}\right) & =\frac{1}{2} e^{i j k} e_{r^{\prime} l^{\prime} q^{\prime}}\left(\frac{\partial^{2} x^{r^{\prime}}}{\partial \xi^{k} \partial \xi^{i}} \frac{\partial x^{l^{\prime}}}{\partial \xi^{j}}+\frac{\partial x^{r^{\prime}}}{\partial \xi^{i}} \frac{\partial^{2} x^{l^{\prime}}}{\partial \xi^{k} \partial \xi^{j}}\right) \\
\left(J \beta_{q^{\prime}}^{k}\right)_{, k} & =0
\end{aligned}
$$

O zero é obtido devido a uma dupla contração entre tensores simétricos e o símbolo de permutação anti-simétrico, $e^{i j k}$. Os resultados acima, (A.12) e (A.13), são extremamente importantes para a redução gradiente adjunto (JAMESON; KIM, 2003a; JAMESON; KIM, 2003b).

Além disso, utilizando símbolos de permutação e sua relação com os tensores correspondentes, é possível escrever a equação (A.10) de uma maneira alternativa: 


$$
g^{i k}=\frac{e^{i p q} e^{k l m}}{2 J^{2}} g_{p l} g_{q m}
$$

onde se verifica um resultado conhecido (FLüGGE, 1972) o qual $\operatorname{det}\left(g_{i j}\right) \equiv g=J^{2}$. Em tempo, é interessante notar nas equações (A.12) e (A.14) a fórmula clássica da inversão de matrizes

$$
\mathbf{A}^{-1}=\frac{1}{\operatorname{det}(\mathbf{A})} \mathbf{C}^{T}
$$

onde $\mathbf{C}^{T}$ é a matriz transposta dos cofatores $\mathbf{A}$.

Uma expressão para o cálculo dos símbolos de Christoffel em termos do operador de transformação, pode ser obtida por meio da relação entre esse símbolos e o tensor métrico (FLüGGE, 1972). Substituindo esse último pela sua definição, expandido o resultado e agrupando termos semelhantes, chega-se a:

$$
\begin{aligned}
2 \Gamma_{i j k} & =g_{j k, i}+g_{k i, j}-g_{i j, k} \\
& =\frac{\partial}{\partial \xi^{i}}\left(\frac{\partial x^{p^{\prime}}}{\partial \xi^{j}} \frac{\partial x^{p^{\prime}}}{\partial \xi^{k}}\right)+\frac{\partial}{\partial \xi^{j}}\left(\frac{\partial x^{s^{\prime}}}{\partial \xi^{k}} \frac{\partial x^{s^{\prime}}}{\partial \xi^{i}}\right)-\frac{\partial}{\partial \xi^{k}}\left(\frac{\partial x^{q^{\prime}}}{\partial \xi^{i}} \frac{\partial x^{q^{\prime}}}{\partial \xi^{j}}\right) \\
2 \Gamma_{i j k} & =2 \frac{\partial^{2} x^{p^{\prime}}}{\partial \xi^{i} \xi^{j}} \frac{\partial x^{p^{\prime}}}{\partial \xi^{k}} \\
\Gamma_{i j k} & =\left(\beta_{j}^{p^{\prime}}\right)_{, i} \beta_{k}^{p^{\prime}}
\end{aligned}
$$

Assim, chega-se ao símbolo de Christoffel com o terceiro índice rebaixado.

Duas expressões similares são utilizadas para o cálculo do símbolo com o terceiro índice elevado. A primeira é obtida da equação (A.16), combinando-a com as equações (A.10) e (A.12). Os principais passos nessa derivação são os seguintes:

$$
\begin{aligned}
\Gamma_{k j}^{p} & =\Gamma_{k j l} g^{l p}=g^{l p} \frac{\partial^{2} x^{q^{\prime}}}{\partial \xi^{k} \xi^{j}} \frac{\partial x^{q^{\prime}}}{\partial \xi^{l}} \\
& =\frac{\epsilon^{p s r} \epsilon^{l m n}}{2} g_{s m} g_{r n} \frac{\partial^{2} x^{q^{\prime}}}{\partial \xi^{k} \xi^{j}} \frac{\partial x^{q^{\prime}}}{\partial \xi^{l}} \\
& =\frac{\epsilon^{p s r} \epsilon^{l m n}}{2} \frac{\partial x^{i^{\prime}}}{\partial \xi^{s}} \frac{\partial x^{i^{\prime}}}{\partial \xi^{m}} \frac{\partial x^{v^{\prime}}}{\partial \xi^{r}} \frac{\partial x^{v^{\prime}}}{\partial \xi^{n}} \frac{\partial x^{q^{\prime}}}{\partial \xi^{l}} \frac{\partial^{2} x^{q^{\prime}}}{\partial \xi^{k} \xi^{j}} \\
& =\frac{\epsilon^{p s r} \epsilon^{q^{\prime} i^{\prime} v^{\prime}}}{2} \frac{\partial x^{i^{\prime}}}{\partial \xi^{s}} \frac{\partial x^{v^{\prime}}}{\partial \xi^{r}} \frac{\partial^{2} x^{q^{\prime}}}{\partial \xi^{k} \xi^{j}} \\
& =\frac{e^{p s r} e_{q^{\prime} i^{\prime} v^{\prime}}}{2 J} \frac{\partial x^{i^{\prime}}}{\partial \xi^{s}} \frac{\partial x^{v^{\prime}}}{\partial \xi^{r}} \frac{\partial^{2} x^{q^{\prime}}}{\partial \xi^{k} \xi^{j}} \\
\Gamma_{k j}^{p} & =\beta_{q^{\prime}}^{p}\left(\beta_{j}^{q^{\prime}}\right)_{, k}
\end{aligned}
$$

aqui, utilizou-se da igualdade entre tensor e símbolo de permutação no sistema de coordenadas cartesianas, bem como a equivalência entre componentes covariante e contravariante, nesse mesmo sistema. Finalmente, utilizando a equação (A.17) 
para obter a última das expressões:

$$
\begin{aligned}
\left(\delta_{j}^{p}\right)_{, k}=\left(\beta_{q^{\prime}}^{p} \beta_{j}^{q^{\prime}}\right)_{, k} & =0 \\
\left(\beta_{q^{\prime}}^{p}\right)_{, k} \beta_{j}^{q^{\prime}}+\Gamma_{k j}^{p} & =0 \\
\Gamma_{k j}^{p} & =-\left(\beta_{q^{\prime}}^{p}\right)_{, k} \beta_{j}^{q^{\prime}}
\end{aligned}
$$

Um resultado importante diz respeito à contração entre dois dos índices do símbolo de Christoffel. Como ponto de partida para isso, utiliza-se a relação entre esses entes e o tensor métrico que aparecem na primeira linha da equação (A.16):

$$
\begin{aligned}
& 2 \Gamma_{i j}^{l}=2 \Gamma_{i j k} g^{l k}=g^{l k}\left(g_{j k, i}+g_{k i, j}-g_{i j, k}\right) \\
& 2 \Gamma_{j i}^{i}=g^{i k}\left(g_{j k, i}+g_{k i, j}-g_{i j, k}\right) \\
& 2 \Gamma_{j i}^{i}=g^{i k} g_{j k, i}+g^{i k} g_{k i, j}-g^{i k} g_{j k, i} \\
& 2 \Gamma_{j i}^{i}=g^{i k} g_{k i, j}
\end{aligned}
$$

Ao passar da segunda para a terceira linha, o produto foi expandido e os índices contraídos do terceiro termo do lado direito da equação foram permutados, revelando, assim, uma similaridade em relação ao primeiro. Ao cancelá-los, chega-se ao resultado final. Neste ponto, a equação (A.14) pode ser substituída por $g^{i k}$ na equação (A.19), chegando em:

$$
2 \Gamma_{j i}^{i}=\frac{1}{J^{2}}\left(\frac{1}{2} e^{i p q} e^{k l m} g_{p l} g_{q m} g_{k i, j}\right)
$$

O termo entre parênteses foi separado para efeito de comparação com a derivada parcial do determinante de $g_{i j}: g=\operatorname{det}\left(g_{i j}\right)=J^{2}$. O símbolo $g$ será utilizado apenas por conveniência algébrica. Este determinante pode ser calculado pela fórmula (FLüGGE, 1972):

$$
g=\frac{1}{6} e^{i p q} e^{k l m} g_{i k} g_{p l} g_{q m}
$$

Assim sendo, faz-se:

$$
\begin{aligned}
g_{, j} & =\frac{1}{6} e^{i p q} e^{k l m}\left(g_{i k} g_{p l} g_{q m}\right)_{, j} \\
& =\frac{1}{6} e^{i p q} e^{k l m}\left(g_{i k, j} g_{p l} g_{q m}+g_{i k} g_{p l, j} g_{q m}+g_{i k} g_{p l} g_{q m, j}\right) \\
& =\frac{1}{6}\left(e^{i p q} e^{k l m}+e^{p i q} e^{l k m}+e^{q p i} e^{m l k}\right) g_{i k, j} g_{p l} g_{q m} \\
g_{, j} & =\frac{1}{2} e^{i p q} e^{k l m} g_{p l} g_{q m} g_{i k, j}
\end{aligned}
$$

Novamente, ao passar da segunda para a terceira linham os índices contraídos foram trocados convenientemente. É possível notar na equação final que os ín- 
dices sobrescritos dos símbolos de permutação nos últimos dois termos sofreram permutações ímpares, se comparados aos da primeira equação. Enquanto isso faz com que haja mudança nos sinais desses símbolos, não há interferência no resultado, já que as permutações ocorrem aos pares e ambos os elementos em cada par sofre a mesma mudança.

Por fim, substituindo o termo entre parênteses da equação (A.20) pela equação (A.22) e, ainda, substituindo o valor de $J^{2}$ por $g$, chega-se a:

$$
\Gamma_{j i}^{i}=\frac{1}{J} \frac{\partial J}{\partial \xi^{j}}
$$

Vale recordar que os tensores métrico e de permutação possuem derivada covariante nula (FLüGGE, 1972):

$$
\left.g^{i j}\right|_{k}=\left.g_{i j}\right|_{k}=\left.\epsilon^{i j k}\right|_{l}=\left.\epsilon_{i j k}\right|_{l}=0
$$

Importante salientar que isso não implica que as derivadas parciais desse entes são nulas. Os resultados obtidos acima permitem que sejam feitas uma série de simplificações na derivada covariante. Uma simplificação importante é a redução do operador divergente, o que é feito por meio da equação (A.23). De volta à primeira equação de (A.7):

$$
\left.u^{i}\right|_{i}=u_{, i}^{i}+u^{k} \Gamma_{i k}^{i}=\frac{1}{J}\left(J u^{i}\right)_{, i}
$$

Da última linha da equação (A.8):

$$
\left.A^{i j}\right|_{j}=A_{, j}^{i j}+A^{l j} \Gamma_{j l}^{i}+A^{i l} \Gamma_{j l}^{j}=\frac{1}{J}\left(J A^{i j}\right)_{, j}+A^{l j} \Gamma_{j l}^{i}
$$

As equações (A.25) e (A.26) são bem conhecidas na literatura. Outras identidades, mais específicas para as aplicações deste trabalho, podem ser provadas. Da equação (A.17),

$$
\begin{aligned}
\beta_{r^{\prime}}^{s}\left(\beta_{n}^{r^{\prime}} u^{n}\right)_{, k} & =\beta_{r^{\prime}}^{s} \beta_{n}^{r^{\prime}}\left(u_{, k}^{n}\right)+u^{n} \beta_{r^{\prime}}^{s}\left(\beta_{n}^{r^{\prime}}\right)_{, k} \\
& =\delta_{n}^{s}\left(u_{, k}^{n}\right)+u^{n} \Gamma_{k n}^{s} \\
& =\left.u^{s}\right|_{k}
\end{aligned}
$$

A equação acima fornece um meio de expressar a derivada covariante em termos do operador de transformação e de derivadas parciais. Pode-se utilizar a equação (A.18) para obter a seguinte expressão;

$$
\beta_{s}^{r^{\prime}}\left(\beta_{r^{\prime}}^{n} u_{n}\right)_{, k}=\left.u_{s}\right|_{k}
$$


A equação (A.17) também permite simplificar o divergente dos tensores de segunda ordem em termos dos componente contravariantes. Da equação: (A.26):

$$
\begin{aligned}
\left.A^{i j}\right|_{j} & =\frac{1}{J}\left(J A^{i j}\right)_{, j}+A^{l j} \Gamma_{j l}^{i} \\
& =\frac{1}{J}\left(J A^{i j}\right)_{, j}+A^{l j} \beta_{k^{\prime}}^{i}\left(\beta_{l}^{k^{\prime}}\right)_{, j} \\
\left.A^{i j}\right|_{j} & =\frac{1}{J} \beta_{k^{\prime}}^{i}\left(\beta_{l}^{k^{\prime}} J A^{l j}\right)_{, j}
\end{aligned}
$$

A última linha da equação (A.29) pode ser utilizada para resgatar a anterior simplemente derivando-a em relação a $\xi^{j}$.

Os resultados acima, em especial as equações (A.25)-(A.29), serão utilizados para derivar as equações de Navier Stokes na forma reduzida.

\section{A.3 Forma Reduzida da Equação de Navier-Stokes}

Cada equação do conjunto (A.5)-(A.6) será trablalhada separadamente. Iniciando pela equação da continuidade, pode-se substituir a equação (A.25) para o divergente para obter:

$$
\partial_{o}(J \rho)+\left(J \rho u^{k}\right)_{, k}=0
$$

posto que os termos relativos à métrica não são tempo dependentes nas aplicações aqui descritas. A equação (A.30) é a forma reduzida da equação da continuidade.

A equação de quantidade de movimento será tratada agora. A equação (A.29) deve ser substituida para os termos divergentes. Assim, multiplica-se essa equação por $J \beta_{i}^{r^{\prime}}$, que é independente do tempo embora dependa de $\xi^{k}$ e obtém-se.

$$
\begin{aligned}
\partial_{o}\left(\rho u^{i}\right)+\left.\left(\rho u^{i} u^{j}\right)\right|_{j}+\left.g^{i j} p\right|_{j} & =\left.\tau^{i j}\right|_{j} \\
\partial_{o}\left(\rho u^{i}\right)+\frac{1}{J} \beta_{k^{\prime}}^{i}\left(\beta_{l}^{k^{\prime}} J u^{l} u^{j}\right)_{, j}+g^{i j} p_{, j} & =\frac{1}{J} \beta_{k^{\prime}}^{i}\left(\beta_{l}^{k^{\prime}} J \tau^{l j}\right)_{, j} \\
\partial_{o}\left(J \rho u^{r^{\prime}}\right)+\delta_{k^{\prime}}^{r^{\prime}}\left(J u^{k^{\prime}} u^{j}\right)_{, j}+J \beta_{i}^{r^{\prime}} g^{i p} \beta_{p}^{n^{\prime}} \beta_{n^{\prime}}^{j} p_{, j} & =\delta_{k^{\prime}}^{r^{\prime}}\left(\beta_{l}^{k^{\prime}} J \tau^{l j}\right)_{, j} \\
\partial_{o}\left(J \rho u^{r^{\prime}}\right)+\left[J \beta_{n^{\prime}}^{j}\left(u^{r^{\prime}} u^{n^{\prime}}+g^{r^{\prime} n^{\prime}} p\right)\right]_{, j} & =\left(\beta_{l}^{r^{\prime}} J \tau^{l j}\right)_{, j}
\end{aligned}
$$

Aqui, utilizou-se da identidade $\beta_{p}^{n^{\prime}} \beta_{n^{\prime}}^{j}=\delta_{p}^{j}$, da equação (A.13) e do fato de que o delta de Kronecker independe de espaço e tempo. A mesma propriedade é válida para o tensor métrico em coordenadas cartesianas, uma vez que $g^{r^{\prime} n^{\prime}} \equiv \delta_{n^{\prime}}^{r^{\prime}}$.

Antes de dar seguimento com a equação (A.31), é conveniente simplificar a expressão do tensor das tensões. Isso é feito substituindo as equações (A.25) e 
(A.27) pelos termos correspodentens:

$$
\begin{aligned}
\tau^{l j} & =\mu\left[\left(\left.u^{l}\right|^{j}+\left.u^{j}\right|^{l}\right)-\left.\frac{2}{3} g^{l j} u^{k}\right|_{k}\right] \\
& =\mu\left[\beta_{s^{\prime}}^{l}\left(\beta_{n}^{s^{\prime}} u^{n}\right)_{, p} g^{p j}+\beta_{s^{\prime}}^{j}\left(\beta_{n}^{s^{\prime}} u^{n}\right)_{, p} g^{p l}-\frac{2 g^{l j}}{3 J}\left(J u^{p}\right)_{, p}\right]
\end{aligned}
$$

Multiplicando-se a equação resultante por $J \beta_{l}^{r^{\prime}}$, chega-se a:

$$
\begin{aligned}
J \beta_{l}^{r^{\prime}} \tau^{l j} & =\mu\left[J\left(u^{r^{\prime}}\right)_{, p} g^{p j}+J \beta_{s^{\prime}}^{j}\left(u^{s^{\prime}}\right)_{, p} \beta_{l}^{r^{\prime}} g^{p l}-\frac{2 \beta_{l}^{r^{\prime}} g^{l j}}{3}\left(J u^{p}\right)_{, p}\right] \\
& =\mu\left[J\left(u^{r^{\prime}}\right)_{, p} \beta_{n^{\prime}}^{p} g^{n^{\prime} s^{\prime}} \beta_{s^{\prime}}^{j}+J \beta_{s^{\prime}}^{j}\left(u^{s^{\prime}}\right)_{, p} \beta_{n^{\prime}}^{p} g^{r^{\prime} n^{\prime}}-\frac{2 g^{r^{\prime} s^{\prime}} \beta_{s^{\prime}}^{j}}{3}\left(J \beta_{n^{\prime}}^{p} u^{n^{\prime}}\right)_{, p}\right] \\
& =\mu\left[J \beta_{s^{\prime}}^{j}\left(u_{, n^{\prime}}^{r^{\prime}} g^{n^{\prime} s^{\prime}}+u_{,{ }^{\prime}}^{s^{\prime}} g^{r^{\prime} n^{\prime}}-\frac{2}{3} u_{,{ }^{\prime}}^{n^{\prime}} g^{r^{\prime} s^{\prime}}\right)\right] \\
J \beta_{l}^{r^{\prime}} \tau^{l j} & =J \beta_{s^{\prime}}^{j} \tau^{r^{\prime} s^{\prime}}
\end{aligned}
$$

O resultado é uma função do tensor das tensões em coordenadas cartesianas, o que já era esperado.

Finalmente substituindo a equação (A.33) para o termo correspondente na equação (A.31) e utilizando a equação (A.13), chega-se a:

$$
\partial_{o}\left(J \rho u^{r^{\prime}}\right)+J \beta_{n^{\prime}}^{j}\left(u^{r^{\prime}} u^{n^{\prime}}+g^{r^{\prime} n^{\prime}} p-\tau^{r^{\prime} n^{\prime}}\right)_{, j}=0
$$

que corresponde a forma reduzida da equação de momento.

Por fim, rearranjando o conjunto de equações governantes do escoamento com as equações (A.30) e (A.34), chega-se a:

$$
\begin{cases}\partial_{o}(J \rho)+J \beta_{q^{\prime}}^{k}\left(\rho u^{q^{\prime}}\right)_{, k} & =0 \\ \partial_{o}\left(J \rho u^{r^{\prime}}\right)+J \beta_{n^{\prime}}^{j}\left(u^{r^{\prime}} u^{n^{\prime}}+g^{r^{\prime} n^{\prime}} p-\tau^{r^{\prime} n^{\prime}}\right)_{, j} & =0\end{cases}
$$

que representa a forma reduzida das equações de Navier-Stokes generalizadas que é amplamente difundida na literatura (JAMESON; MARTINELLI; PIERCE, 1998). Nota-se que o conjunto acima envolve tanto o sistema cartesiano de coordenadas quanto o transformado, como fora mencionado anteriormente. Para escoamentos incompressíveis a equação da continuidade impõe a condição de divergência nula no campo de velocidades.

$$
\left.u^{i}\right|_{i}=0
$$

Como resultado, o tensor das tensões (A.6) é reduzido a:

$$
\tau^{i j}=\mu\left(\left.u^{i}\right|^{j}+\left.u^{j}\right|^{i}\right)
$$


Calculando a divergência de $\tau$, da maneira a qual ela aparece na equação do momento, uma outra simplificação é possível:

$$
\begin{aligned}
\left.\tau^{i j}\right|_{j} & =\mu\left(u^{i}\left|{ }^{j}\right|_{j}+\left.\left.u^{j}\right|^{i}\right|_{j}\right) \\
& =\left.\left.\mu u^{i}\right|^{j}\right|_{j}
\end{aligned}
$$

uma vez que a ordem das derivadas covariantes podem ser trocadas, como já apresentado, isso implica que $\left.\left.u^{j}\right|_{j}\right|^{i}=0$.

Fazendo esa simplificação no conjunto de equações (A.5)-(A.6), isso o reduz a:

$$
\left\{\begin{aligned}
\left.u^{i}\right|_{i} & =0 \\
\partial_{o} u^{i}+\left.\left(u^{i} u^{j}+g^{i j} p v\right)\right|_{j} & =\left.\nu u^{i}{ }^{j}\right|_{j}
\end{aligned}\right.
$$

Faz-se a mesma simplificação ao conjunto (A.35):

$$
\left\{\begin{array}{r}
\left(J \beta_{q^{\prime}}^{k} q^{q^{\prime}}\right)_{, k}=0 \\
\partial_{o}\left(J u^{r^{\prime}}\right)+\left\{J \beta_{n^{\prime}}^{j}\left[u^{r^{\prime}} u^{n^{\prime}}+g^{r^{\prime} n^{\prime}} p v-\nu\left(u_{, p^{\prime}}^{r^{\prime}}\right) g^{p^{\prime} n^{\prime}}\right]\right\}_{, j}=0
\end{array}\right.
$$

onde o termos relativos à métrica foram colocados dentro da derivada por conveniência algébrica. Aqui, mais uma vez, utilizou-se a equação (A.13).

\section{A.4 Conclusões}

A derivação para obter o conjunto de equações de Navier-Stokes reduzidas (A.35) partindo do conjunto estendido (A.5) foi um processo puramente algébrico. Um ponto importante a ser ressaltado é o fato de ambos os conjuntos, reduzido e estendido, serem equivalentes do ponto de vista físico.

Comparando-se o conjunto reduzido com o estendido, nota-se a simplicidade do primeiro em relação ao segundo, explicando, um pouco, o porquê da forma reduzida ser facilmente encontrada na literatura. No conjunto reduzido há poucos termos relativos à métrica e os existentes são explícitos. Isso facilita o trato algébrico bem como o cálculo de gradientes. Com isso conclui-se que o conjunto reduzido é mais vantajoso de se trabalhar do que o estendido.

Diante de tantas vantagens do conjunto reduzido, vale apontar uma vantagem do conjunto estendido. Sua forma expressa em termos de divergentes facilita a 
aplicação do Teorema de Gauss na sua forma generalizada:

$$
\left.\int_{\mathcal{D}} u^{k}\right|_{k} \mathrm{~d} V=\oint_{\partial \mathcal{D}} u^{k} n_{k} \mathrm{~d} A
$$

E isso é um passo fundamental para todo o desenvolvimento do problema adjunto. 


\section{APÊNDICE B - RELAÇÕES ENTRE Elementos de Área dos EspaÇos Físico E TRANSFORMADO}

Partindo-se de uma superfície genérica em R3, $S^{\prime}$, expressa por meio de coordenadas cartesianas $x^{3^{\prime}}=x^{3^{\prime}}\left(x^{1^{\prime}}, x^{2^{\prime}}\right)$. Do Cálculo diferencial e integral, o elemento de área dessa superfície é dado por (KAPLAN, 1971; PISKUNOV, 1968):

$$
\mathrm{d} S^{\prime}=\sqrt{1+\left(\frac{\partial x^{3^{\prime}}}{\partial x^{1^{\prime}}}\right)^{2}+\left(\frac{\partial x^{3^{\prime}}}{\partial x^{2^{\prime}}}\right)^{2}} \mathrm{~d} x^{1^{\prime}} \mathrm{d} x^{2^{\prime}}
$$

Assumindo que a superfície $S^{\prime}$ pode ser transformada em um plano constante $S$ no espaço transformado $\left(S^{\prime} \Leftrightarrow S: \xi^{3}=0\right)$ e que a transformação é definida como na equação (A.2):

$$
J=\operatorname{det}\left(\beta_{i}^{j^{\prime}}\right)=\frac{\partial\left(x^{1^{\prime}}, x^{2^{\prime}}, x^{3^{\prime}}\right)}{\partial\left(\xi^{1}, \xi^{2}, \xi^{3}\right)}
$$

Assim, o elemento de área nesse sistema em particular será dado por $\mathrm{d} S=\mathrm{d} \xi^{1} \mathrm{~d} \xi^{2}$. Sob essas condições a relação entre os elementos de área dos espaços físico e transformado é dada por:

$$
\begin{aligned}
\left|\frac{\mathrm{d} S^{\prime}}{\mathrm{d} S}\right| \mathrm{d} S & =\sqrt{1+\left(\frac{\partial x^{3^{\prime}}}{\partial x^{1^{\prime}}}\right)^{2}+\left(\frac{\partial x^{3^{\prime}}}{\partial x^{2^{\prime}}}\right)^{2}} \frac{\partial\left(x^{1^{\prime}}, x^{2^{\prime}}\right)}{\partial\left(\xi^{1}, \xi^{2}\right)} \mathrm{d} \xi^{1} \mathrm{~d} \xi^{2} \\
\left|\frac{\mathrm{d} S^{\prime}}{\mathrm{d} S}\right| & =\sqrt{1+\left(\frac{\partial x^{3^{\prime}}}{\partial x^{1^{\prime}}}\right)^{2}+\left(\frac{\partial x^{3^{\prime}}}{\partial x^{2^{\prime}}}\right)^{2}} \frac{\partial\left(x^{1^{\prime}}, x^{2^{\prime}}\right)}{\partial\left(\xi^{1}, \xi^{2}\right)}
\end{aligned}
$$

O Jacobiano bidimensional do lado direito da equação (B.3) pode ser tratato como o determinante que é obtido ao suprimir a terceira linha e a terceira coluna do Jacobiano da transformação (B.2). Por fim, através da regra da cadeia e das definições $\beta_{j^{\prime}}^{i}$ e $\beta_{i}^{j^{\prime}}$, da equação (A.2), obtem-se:

$$
\left|\frac{\mathrm{d} S^{\prime}}{\mathrm{d} S}\right|=\sqrt{1+\left(\frac{\beta_{1^{\prime}}^{3}}{\beta_{3^{\prime}}^{3}}\right)^{2}+\left(\frac{\beta_{2^{\prime}}^{3}}{\beta_{3^{\prime}}^{3}}\right)^{2}} \frac{\operatorname{Cof}\left(\beta_{3}^{3^{\prime}}\right)}{\beta_{3}^{3^{\prime}}}
$$


onde o símbolo Cof $\left(\beta_{3}^{3^{\prime}}\right)$ denota o cofator do elemento $\beta_{3}^{3^{\prime}}$. A equação (B.4) é válida para valores de $\beta_{3}^{3^{\prime}}$ e $\beta_{3}^{3^{\prime}}$ não nulos.

Para o problema bidimensional, considera-se uma fronteira descrita por $S^{\prime}$ : $x^{2^{\prime}}=x^{2^{\prime}}\left(x^{1^{\prime}}\right)$, em coordenadas cartesianas. Assumindo que essa curva pode ser transformada em uma reta no espaço transformado, pode-se escrever:

$$
S^{\prime} \Leftrightarrow S: \xi^{2}\left(x^{1^{\prime}}, x^{2^{\prime}}\right)=0 \Leftrightarrow\left\{\begin{array}{l}
x^{1^{\prime}}=x^{1^{\prime}}\left(\xi^{1}, 0\right) \\
x^{2^{\prime}}=x^{2^{\prime}}\left(\xi^{1}, 0\right)
\end{array}\right.
$$

Neste caso, um elemento infinitesimal da fronteira é dado, em coordenadas cartesianas, por:

$$
\left|\mathrm{d} S^{\prime}\right|=\sqrt{1+\left(\frac{\partial x^{2^{\prime}}}{\partial x^{1^{\prime}}}\right)^{2}}\left|\mathrm{~d} x^{1^{\prime}}\right|
$$

e, no espaço transformado, o elemento de fronteira é dado por $\mathrm{d} S=\mathrm{d} \xi^{1}$. Assim, seguindo a mesma abordagem acima, chega-se em:

$$
\begin{aligned}
\left|\frac{\mathrm{d} S^{\prime}}{\mathrm{d} \xi^{1}}\right| \mathrm{d} \xi^{1} & =\sqrt{1+\left(\frac{\partial x^{2^{\prime}} / \partial \xi^{1}}{\partial x^{1^{\prime}} / \partial \xi^{1}}\right)^{2}} \frac{\partial x^{1^{\prime}}}{\partial \xi^{1}} \mathrm{~d} \xi^{1} \\
\left|\frac{\mathrm{d} S^{\prime}}{\mathrm{d} \xi^{1}}\right| & =\sqrt{\left(\frac{\partial x^{1^{\prime}}}{\partial \xi^{1}}\right)^{2}+\left(\frac{\partial x^{2^{\prime}}}{\partial \xi^{1}}\right)^{2}}=\sqrt{g_{11}}
\end{aligned}
$$

onde o resultado coincide com o componente covariante do tensor métrico $g_{i j}$. 


\section{Apêndice C - Testes de Convergência DE MALHAS}

Neste apêndice são apresentados os testes de convergência feitos para encontrar as melhores malhas para as simulações de modo a garantir a confiabilidade das soluções numéricas. Desse modo, realizaram-se testes de convergência em relação a alguns parâmetros de simulação para ambos os códigos computacionais utilizados no trabalho: SEMTEX e FreeFem ++ .

Como ponto de partida, para cada código, uma malha foi elaborada com determinados parâmetros e, em seguida, foram feitos os testes variando cada um desses parâmetros separadamente. Como cada código computacional tem suas particularidades, os testes não são iguais para ambos e as diferenças serão apresentadas a seguir.

\section{C.1 SEMTEX}

Iniciando os testes pelo SEMTEX, lista-se os parâmetros de simulação que são avaliados: quantidade de iterações $(\mathrm{N})$, grau do polinômio interpolador $(\mathrm{P}) \mathrm{e}$ o passo de integração temporal $(\Delta t)$.

\section{C.1.1 Análise da Quantidade de Iterações}

O primeiro parâmetro a ser testado é a quantidade de iterações. É importante que o tempo de simulação $(N \times \Delta t)$ seja suficientemente grande de modo a desprezar o transiente inicial da simulação. As simulações iniciais foram realizadas com $P=10$ e $\Delta t=0,001$ e os resultados obtidos (variando-se apenas $N$ ) estão exibidos a seguir: 


\section{C.1.1.1 Malha para o Escoamento Através de um Canal Bidimensio- nal}

Tabela C.1: Teste de Convergência - Número de Iterações - Canal Bidimensional

\begin{tabular}{|c|c|c|}
\hline Teste & $\mathbf{N}$ & $\boldsymbol{C}_{\boldsymbol{d}}$ \\
\hline 1 & 100 & 2,01896 \\
\hline 2 & 1000 & 1,60654 \\
\hline 3 & 7000 & 1,60652 \\
\hline 4 & 10000 & 1,60652 \\
\hline
\end{tabular}

\section{C.1.1.2 Malha para o Escoamento Através de um Canal Bidimensio- nal com Variação de Espessura}

Tabela C.2: Teste de Convergência - Número de Iterações - Canal Bidimensional com Variação de Espessura

\begin{tabular}{|c|c|c|}
\hline Teste & $\mathbf{N}$ & $\boldsymbol{C}_{\boldsymbol{d}}$ \\
\hline 1 & 100 & 2,07212 \\
\hline 2 & 1000 & 1,83821 \\
\hline 3 & 7000 & 1,82889 \\
\hline 4 & 10000 & 1,82889 \\
\hline
\end{tabular}

Analisando os dados, conclui-se que para o canal bidimensional, simulando até $N=7000$ o transiente inicial será desprezado e o escoamento simulado já se encontra em regime permanente. Para o canal bidimensional com variação de espessura, simular-se-á também até $N=7000$.

\section{C.1.2 Grau do Polinômio Interpolador}

O segundo parâmetro a ser testado é o grau do polinômio interpolador. Como o SEMTEX é baseado no Método dos Elementos Espectrais, uma das maneiras de aumentar a precisão da solução é o refinamento espacial tipo $P$ que consiste, justamente, em aumentar a ordem do polinomio interpolador. Quanto maior o valor de $P$ haverá mais pontos de colocação dentro de cada elemento melhorando, assim, a precisão da solução. Entretanto, quanto maior o valor de $P$ maior o custo computacional então convém encontrar o melhor valor de $P$ para que a solução seja o mais precisa quanto se deseja porém sem comprometer significativamente o custo computacional. As simulações iniciais foram realizadas com $N=7000$ e $\Delta t=0,001$ e os resultados obtidos (variando-se apenas $P$ ) estão exibidos a seguir: 


\section{C.1.2.1 Malha para o Escoamento Através de um Canal Bidimensio- nal}

Tabela C.3: Teste de Convergência - Grau do Polinômio - Canal Bidimensional

\begin{tabular}{|c|c|c|}
\hline Teste & $\mathbf{P}$ & $\boldsymbol{C}_{\boldsymbol{d}}$ \\
\hline 1 & 3 & 1,60467 \\
\hline 2 & 7 & 1,60617 \\
\hline 3 & 10 & 1,60652 \\
\hline 4 & 12 & 1,60664 \\
\hline
\end{tabular}

\section{C.1.2.2 Malha para o Escoamento Através de um Canal Bidimensio- nal com Variação de Espessura}

Tabela C.4: Teste de Convergência - Grau do Polinômio - Canal Bidimensional com Variação de Espessura

\begin{tabular}{|c|c|c|}
\hline Teste & $\mathbf{P}$ & $\boldsymbol{C}_{\boldsymbol{d}}$ \\
\hline 1 & 3 & 1,82646 \\
\hline 2 & 10 & 1,82889 \\
\hline 3 & 12 & 1,82904 \\
\hline
\end{tabular}

Analisando os dados, conclui-se que, para o canal bidimensional, simulandose com $P=10$ obtém-se uma precisão aceitável (erro inferior a 0,01\%). Para o canal bidimensional com variação de espessura, utilizar-se-á também $P=10$

\section{C.1.3 Passo de Integração Temporal}

O último parâmetro a ser testado é o passo de integração temporal $\Delta t$. Como visto na equação (4.30) parte do termo difusivo é tratada de forma explícita, com isso o passo de integração deve ser pequeno o bastante para garantir a convergência da solução (Condição de Courant-Friedrich-Levy) mas não pequeno a ponto de elevar demasiadamente o custo computacional. As simulações iniciais foram realizadas com $N=7000$ e $P=10$ e os resultados obtidos (variando-se apenas $\Delta t$ ) estão exibidos a seguir:

\section{C.1.3.1 Malha para o Escoamento Através de um Canal Bidimensio- nal}


Tabela C.5: Teste de Convergência - Passo de Integração - Canal Bidimensional

\begin{tabular}{|c|c|c|}
\hline Teste & $\boldsymbol{\Delta} \boldsymbol{t}$ & $\boldsymbol{C}_{\boldsymbol{d}}$ \\
\hline 1 & 0,01 & 1,60654 \\
\hline 2 & 0,001 & 1,60652 \\
\hline 3 & 0,0005 & 1,60652 \\
\hline
\end{tabular}

\section{C.1.3.2 Malha para o Escoamento Através de um Canal Bidimensio- nal com Variação de Espessura}

Tabela C.6: Teste de Convergência - Passo de Integração - Canal Bidimensional com Variação de Espessura

\begin{tabular}{|c|c|c|}
\hline Teste & $\boldsymbol{\Delta} \boldsymbol{t}$ & $\boldsymbol{C}_{\boldsymbol{d}}$ \\
\hline 1 & 0,01 & 1,82876 \\
\hline 2 & 0,001 & 1,82890 \\
\hline 3 & 0,0005 & 1,82891 \\
\hline
\end{tabular}

Analisando os dados, conclui-se que, para o canal bidimensional, simulando com $\Delta t=0,001$ a solução está convergida e este será o passo adotado para as simulações. O mesmo é válido para o canal bidimensional com variação de espessura,

\section{C.2 FreeFem ++}

Para o FreeFem ++ os parâmetros de simulação são outros. Como este código é utilizado para resolver a equação correspondente ao estado estacionário, não há número de iterações nem passo de integração para ser avaliado. E visto que o código fornece poucas opções de refinamento através do aumento do grau do polinômio interpolador, o único parâmetro que será avaliado é a quantidade de elementos $(E)$ na horizontal e na vertical. Será utilizado

\section{C.2.1 Quantidade de Elementos}

Quanto mais elementos distribuídos nas fronteiras horizontal e vertical, melhor a precisão da solução e maior o custo computacional. Deseja-se, então, encontrar uma quantidade de elementos que faça com que a solução seja precisa mas que não eleve muito o custo computacional. As simulações iniciais foram realizadas com $P=4$ que é o máximo grau possível no FreeFem ++ e os resultados obtidos estão exibidos a seguir: 


\section{C.2.1.1 Malha para o Escoamento Através de um Canal Bidimensio- nal}

Quantidade de Elementos na Entrada $N_{\text {entrada }}$

Tabela C.7: Teste de Convergência - $N_{\text {entrada }}$ - Canal Bidimensional

\begin{tabular}{|c|c|c|}
\hline Teste & $\boldsymbol{N}_{\text {entrada }}$ & $\boldsymbol{C}_{\boldsymbol{d}}$ \\
\hline 1 & 100 & 1,60617 \\
\hline 2 & 200 & 1,60612 \\
\hline 3 & 400 & 1,60609 \\
\hline
\end{tabular}

$\underline{\text { Quantidade de Elementos na Saída } N_{\text {saida }}}$

Tabela C.8: Teste de Convergência - $N_{\text {saida }}$ - Canal Bidimensional com Variação de Espessura

\begin{tabular}{|c|c|c|}
\hline Teste & $\boldsymbol{N}_{\text {saida }}$ & $\boldsymbol{C}_{\boldsymbol{d}}$ \\
\hline 1 & 100 & 1,60606 \\
\hline 2 & 400 & 1,60609 \\
\hline 3 & 600 & 1,60609 \\
\hline
\end{tabular}

Quantidade de Elementos nas fronteiras Superior e Inferior $N_{\text {topo/baixo }}$

Tabela C.9: Teste de Convergência - $N_{\text {topo/baixo }}$ - Canal Bidimensional com Variação de Espessura

\begin{tabular}{|c|c|c|}
\hline Teste & $\boldsymbol{N}_{\text {topo/baixo }}$ & $\boldsymbol{C}_{\boldsymbol{d}}$ \\
\hline 1 & 200 & 1,60552 \\
\hline 2 & 400 & 1,60609 \\
\hline 3 & 600 & 1,60634 \\
\hline
\end{tabular}

\section{C.2.1.2 Malha para o Escoamento Através de um Canal Bidimensio- nal com Variação de Espessura}

Quantidade de Elementos na Entrada $N_{\text {entrada }}$

Tabela C.10: Teste de Convergência - $N_{\text {entrada }}$ - Canal Bidimensional com Variação de Espessura

\begin{tabular}{|c|c|c|}
\hline Teste & $\boldsymbol{N}_{\text {entrada }}$ & $\boldsymbol{C}_{\boldsymbol{d}}$ \\
\hline 1 & 100 & 1,79781 \\
\hline 2 & 200 & 1,79795 \\
\hline 3 & 400 & 1,79797 \\
\hline
\end{tabular}


$\underline{\text { Quantidade de Elementos na Saída } N_{\text {saida }}}$

Tabela C.11: Teste de Convergência - $N_{\text {saida }}$ - Canal Bidimensional

\begin{tabular}{|c|c|c|}
\hline Teste & $\boldsymbol{N}_{\text {saida }}$ & $\boldsymbol{C}_{\boldsymbol{d}}$ \\
\hline 1 & 100 & 1,7981 \\
\hline 2 & 400 & 1,7979 \\
\hline 3 & 600 & 1,7979 \\
\hline
\end{tabular}

$\underline{\text { Quantidade de Elementos nas fronteiras Superior e Inferior } N_{\text {topo/baixo }}}$

Tabela C.12: Teste de Convergência - $N_{\text {topo/baixo }}$ - Canal Bidimensional

\begin{tabular}{|c|c|c|}
\hline Teste & $\boldsymbol{N}_{\text {topo/baixo }}$ & $\boldsymbol{C}_{\boldsymbol{d}}$ \\
\hline 1 & 200 & 1,7962 \\
\hline 2 & 400 & 1,7980 \\
\hline 3 & 600 & 1,7981 \\
\hline
\end{tabular}

Analisando os resultados exibidos acima, conclui-se que para o canal bidimensional a malha possuirá 200 elementos na entrada, 400 nas fronteiras superior e inferior e 400 elementos na saída. Para o canal bidimensional com variação de espessura, a malha possuirá a mesma quantidade de elementos nas fronteiras que a malha do canal bidimensional. 


\section{APÊNDICE D - SimplificAÇÃO DAS InTEGRAIS Do GRADIENTE ADJunto}

Este apêndice apresenta a simplificação das integrais do gradiente adjunto. Essa simplificação baseia-se no trabalho (JAMESON; KIM, 2003a), no qual as integrais de volume que compõem o gradiente adjunto são transformadas em integrais de superfície. A idéia central por trás desse procedimento consiste no fato de que as variações métricas no domínio resultam somente de variações nas fronteiras do mesmo. Consequentemente é possível expressar, matematicamente, as primeiras em função das segundas. Para tal, os autores definem pseudo-variações físicas (denominação dos autores) que ocorrem única e exclusivamente devido a variações na malha computacional, isto é, com parâmetros de geometria e do escoamento constantes. O trabalho citado implementa esse procedimento para as equações de Euler enquanto aqui serão tratadas as equações da Navier-Stokes.

\section{D.1 Introdução}

A expressão completa do gradiente adjunto está exibida na eq. (5.10),na qual as segunda e terceira integrais serão tratadas nesse apêndice. Antes de iniciar o procedimento é importante definir de maneira clara o que é uma variação métrica. De acordo com a figura A.1, a idéia consiste no seguinte: quando a geometria do corpo do problema é modificada no espaço físico, os operadores de transformação espacial $\beta$ e $\beta^{-1}$ devem mudar da mesma forma. Isso é necessário para garantir que, no espaço transformado (novo espaço físico), contornos e faces dos elementos na malha sejam mapeados para um domínio retangular. Naturalmente, outros termos relativos à métrica do problema serão modificados como consequência desse mapeamento.

Para qualquer parametrização geométrica do corpo, as coordenadas no espaço transformado, $\xi^{i}$, são funções conhecidas das coordenadas do espaço físico, $x^{k^{\prime}}$, e de um número finito de parâmetros, $a^{j}: \xi^{i}=f^{i}\left(x^{k^{\prime}} ; a^{j}\right)$. A forma da função $f^{i}$ 
depende da parametrização adotada. Aqui apenas assume-se que a função é de classe $\mathcal{C}^{2}$ em um domínio aberto $\mathcal{D} \subset \mathbb{R}^{3}$ que contenha o domínio do escoamento e que também seja $\mathcal{C}^{2}$ em relação aos parâmetros $a^{j}$.

Com todos os parâmetros fixos, as funções são definidas por: $f^{i}: \mathcal{D} \rightarrow \mathbb{R}^{3}$, e o teorema da função inversa (SPIVAK, 1995) implica que, dado $x_{c}^{k^{\prime}} \in \mathcal{D}$ | $\operatorname{det}\left[\mathrm{D} f^{i}\left(x_{c}^{k^{\prime}}\right)\right] \neq 0$, resulta em dois conjuntos abertos:

$\mathrm{X} \subset \mathcal{D} \mid x_{c}^{k^{\prime}} \in \mathrm{X}$ e $\Xi \subset \mathbb{R}^{3} \mid \xi_{c}^{i}=f^{i}\left(x_{c}^{k^{\prime}}\right) \in \Xi$, e existem funções $g^{k^{\prime}}: \Xi \rightarrow \mathcal{D}$, $\operatorname{logo} f^{i}\left[g^{k^{\prime}}\left(\xi^{p}\right)\right]=\xi^{i} \forall \xi^{i} \in \Xi$ e $g^{k^{\prime}}\left[f^{i}\left(x^{r^{\prime}}\right)\right]=x^{k^{\prime}} \forall x^{k^{\prime}} \in \mathrm{X}$. Ademais, tem-se que:

$$
\mathrm{D} f^{i} \equiv \frac{\partial f^{i}}{\partial x^{k^{\prime}}}=\frac{\partial \xi^{i}}{\partial x^{k^{\prime}}} \equiv \beta_{k^{\prime}}^{i} \quad \text { e } \quad \mathrm{D} g^{k^{\prime}} \equiv \frac{\partial g^{k^{\prime}}}{\partial \xi^{i}}=\left\{\mathrm{D} f^{i}\left[g^{k^{\prime}}\left(\xi^{p}\right)\right]\right\}^{-1}=\left\{\beta_{k^{\prime}}^{i}\right\}^{-1} \equiv \beta_{i}^{k^{\prime}}
$$

que estabelece a existência de uma transformação inversa e recupera resultados do apêndice A, como esperado. É importante salientar que esse teormea também assegura que $g^{k^{\prime}}$ deve ser diferenciável como $f^{i}$, o que significa $\mathcal{C}^{2}(\Xi ; \mathcal{D})$ neste caso.

Quando se tratam de variações paramétricas, o cenário é um pouco diferente. É necessário, nesse caso, levar em conta um espaço de parâmetros, tal qual $a^{j} \in \mathbb{R}^{p}$, onde $1 \leq j \leq p$. Neste caso, pode-se estabelecer um conjunto aberto: $\Omega \subset \mathbb{R}^{3} \times \mathbb{R}^{p+3}$, tal que $\mathcal{D} \subset \Omega$. Neste conjunto aberto, define-se o vetor $\chi \equiv\left(x^{1^{\prime}}, x^{2^{\prime}}, x^{3^{\prime}} ; a^{1}, \ldots, a^{p}\right)^{T} \mid \chi \in \Omega$, onde $\chi^{r^{\prime}}=x^{r^{\prime}}$ para $1 \leq r^{\prime} \leq 3 \mathrm{e}$ $\chi^{r^{\prime}=j+3}=a^{j}$ para $3<r^{\prime} \leq p$. Dadas essas condições, define-se:

$$
F^{i}\left(\xi^{n}, \chi^{r^{\prime}}\right) \equiv f^{i}\left(\chi^{r^{\prime}}\right)-\xi^{i}=0
$$

Com essa definição e com as propriedades de $f^{i}, F^{i}$ deve ser $\mathcal{C}^{2}\left(\Omega ; \mathbb{R}^{3}\right)$. Assumese ainda que existe um ponto $\left(\xi_{c}, \chi_{c}\right)$ em $\Omega$, onde $\operatorname{det}\left(\partial F^{i} / \partial \xi^{n}\right) \neq 0$. O teorema da função implícita (SPIVAK, 1995) pode ser aplicado para $F^{i}: \Omega \rightarrow \mathbb{R}^{3}$, mesmo com a função $f^{i}$ sendo desconhecida. Isso implica que existem dois conjuntos abertosX $\subset \Omega \mid \chi_{c} \in \mathrm{X}$ and $\Xi \subset \mathbb{R}^{3} \mid \xi_{c} \in \Xi$, tais que:

I. -Para todo $\chi^{r^{\prime}} \in \mathrm{X}$, existe único $\xi^{i}=h^{i}\left(\chi^{r^{\prime}}\right) \in \Xi \mid F^{i}\left[h^{n}\left(\chi^{s^{\prime}}\right), \chi^{r^{\prime}}\right]=0$. Assim, neste caso, a unicidade implica que $h^{i}\left(\chi^{r^{\prime}}\right) \equiv f^{i}\left(\chi^{r^{\prime}}\right)$.

II. -A função implícita $h^{i}: \mathrm{X} \rightarrow \Xi$ é $\mathcal{C}^{2}\left(\mathrm{X} ; \mathbb{R}^{3}\right), \xi_{c}^{i}=h^{i}\left(\chi_{c}^{r^{\prime}}\right)$, e:

$$
\mathrm{D} h^{i} \equiv \underbrace{\left(\frac{\partial h^{i}}{\partial \chi^{r^{c}}}\right)}_{3 \times(3+p)}=-\underbrace{\left\{\frac{\partial F^{n}}{\partial \xi^{i}}\left[h^{m}\left(\chi^{s^{\prime}}\right), \chi^{p^{\prime}}\right]\right\}^{-1}}_{3 \times 3} \cdot \underbrace{\left\{\frac{\partial F^{n}}{\partial \chi^{r^{\prime}}}\left[h^{m}\left(\chi^{s^{\prime}}\right), \chi^{p^{\prime}}\right]\right\}}_{3 \times(3+p)}
$$


Considerando que, neste caso, $h^{i} \equiv f^{i}$ a eq. (D.3) pode ser traduzida como:

$$
\begin{aligned}
\frac{\partial f^{i}}{\partial \chi^{r^{6}}} & =-\left(-\delta_{n}^{i}\right) \frac{\partial F^{n}}{\partial \chi^{r^{\prime}}} \\
\frac{\partial \xi^{i}}{\partial \chi^{r^{6}}} & =(\underbrace{\beta_{r^{\prime}}^{i}}_{1 \leq r^{\prime} \leq 3} \mid \underbrace{\frac{\partial f^{i}}{\partial a^{r^{\prime}-3}}}_{3<r^{\prime} \leq p})_{1 \leq i \leq 3}
\end{aligned}
$$

De onde vêm dois resultados importantes:

$$
\beta_{r^{\prime}}^{i} \equiv \frac{\partial \xi^{i}}{\partial x^{r^{\prime}}}=\frac{\partial f^{i}}{\partial x^{r^{\prime}}} \quad \text { e } \quad \frac{\partial \xi^{i}}{\partial a^{j}}=\frac{\partial f^{i}}{\partial a^{j}}
$$

A primeira equação recupera (D.1), como esperado, porém a segunda é nova. Além disso, elas mostram que, por diferenciação direta, somente, resulta nas derivadas parciais de $\xi^{i}$ em relação a $x^{k^{\prime}}$ e $a^{j}$. Ainda mais importante, é o fato de que $x^{k^{\prime}}$ e $a^{j}$ são independentes no contexto dessa transformação. Em particular, isso implica que $\beta_{r^{\prime}}^{i}=\beta_{r^{\prime}}^{i}\left(x^{k^{\prime}} ; a^{j}\right)$.

Já a transformação inversa é um pouco mais complicada. Parte-se da mesma relação entre $\xi^{i}, x^{k^{\prime}}$ e $a^{j}$ que está por trás de $F^{i}$ na eq. (D.2). Entretanto, as variavéis dependentes são agora $x^{k^{\prime}}$, então a relação deve ser reescrita. Para isso, defini-se um conjunto aberto $\Sigma \subset \mathbb{R}^{3} \times \mathbb{R}^{(p+3)}$, de modo que $\Xi \subset \Sigma$. Assim, define-se o vetor $\zeta \equiv\left(\xi^{1}, \xi^{2}, \xi^{3} ; a^{1}, \ldots, a^{p}\right)^{T} \mid \zeta \in \Sigma$, onde $\zeta^{i}=\xi^{i}$ para $1 \leq i \leq 3 \mathrm{e}$ $\zeta^{i=j+3}=a^{j}$ para $3<i \leq p$. Dadas essas condições, é possível escrever $F^{i}: \Sigma \rightarrow$ $\mathbb{R}^{3}$ na forma:

$$
\begin{aligned}
F^{i}\left(\xi^{n} ; x^{k^{\prime}} ; a^{j}\right) & =f^{i}\left(x^{k^{\prime}}, a^{j}\right)-\xi^{i}=0 \\
F^{i}\left(\zeta^{s} ; x^{k^{\prime}}\right) & =f^{i}\left(x^{k^{\prime}},\left.\zeta^{s}\right|_{3<s \leq p}\right)_{1 \leq i \leq 3}-\left.\zeta^{i}\right|_{1 \leq i \leq 3}=0
\end{aligned}
$$

que é $\mathcal{C}^{2}\left(\Sigma ; \mathbb{R}^{3}\right)$, graças a sua definição e em virtude das propriedades de $f^{i}$. Assim, novamente, assume-se que existe um ponto $\left(x_{c}, \zeta_{c}\right)$ em $\Sigma$, onde $\operatorname{det}\left(\partial F^{i} / \partial x^{k^{\prime}}\right) \neq$ 0. O teorema da função implícita (SPIVAK, 1995) implica que existem dois conjuntos abertos, $\mathrm{Z} \subset \Sigma \mid \zeta_{c} \in \mathrm{Z}$ e $\mathrm{X} \subset \mathbb{R}^{3} \mid x_{c} \in \mathrm{X}$, logo:

I. -Para todo $\zeta^{i} \in \mathrm{Z}$, existe único $x^{k^{\prime}}=g^{k^{\prime}}\left(\zeta^{s}\right) \in \mathrm{X} \mid F^{i}\left[g^{k^{\prime}}\left(\zeta^{s}\right), \zeta^{m}\right]=0$.

II. -A função implícita $g^{k^{\prime}}: \mathrm{Z} \rightarrow \mathrm{X}$ é $\mathcal{C}^{2}\left(\mathrm{Z} ; \mathbb{R}^{3}\right), x_{c}^{k^{\prime}}=g^{k^{\prime}}\left(\zeta_{c}^{s}\right)$, e:

$$
\mathrm{D} g^{k^{\prime}} \equiv \underbrace{\left(\frac{\partial g^{k^{\prime}}}{\partial \zeta^{i}}\right)}_{3 \times(3+p)}=-\underbrace{\left\{\frac{\partial F^{n}}{\partial x^{k^{\prime}}}\left[g^{p^{\prime}}\left(\zeta^{s}\right), \zeta^{m}\right]\right\}^{-1}}_{3 \times 3} \cdot \underbrace{\left\{\frac{\partial F^{n}}{\partial \zeta^{i}}\left[g^{p^{\prime}}\left(\zeta^{s}\right), \zeta^{m}\right]\right\}}_{3 \times(3+p)}
$$


Considerando que $x^{k^{\prime}}=g^{k^{\prime}}\left(\zeta^{s}\right)$, a equação acima. (D.7) leva a:

$$
\begin{aligned}
& \frac{\partial g^{k^{\prime}}}{\partial \zeta^{i}}=-\left(\beta_{k^{\prime}}^{n}\right)^{-1} \frac{\partial F^{n}}{\partial \zeta^{i}} \\
& \frac{\partial x^{k^{\prime}}}{\partial \zeta^{i}}=-\beta_{n}^{k^{\prime}}(\underbrace{-\delta_{i}^{n}}_{1 \leq i \leq 3} \mid \underbrace{\frac{\partial F^{n}}{\partial a^{i-3}}}_{3<i \leq p})_{1 \leq n \leq 3}
\end{aligned}
$$

Da onde obtém-se outro importante par de resultados, para $x^{k^{\prime}}=g^{k^{\prime}}\left(\zeta^{s}\right)=$ $g^{k^{\prime}}\left(\xi^{r} ; a^{p}\right)$ :

$$
\frac{\partial g^{k^{\prime}}}{\partial \xi^{i}}=\beta_{i}^{k^{\prime}}=\frac{\partial x^{k^{\prime}}}{\partial \xi^{i}} \quad \text { e } \frac{\partial g^{k^{\prime}}}{\partial a^{j}}=-\beta_{i}^{k^{\prime}} \frac{\partial F^{i}}{\partial a^{j}} \Leftrightarrow \frac{\partial x^{k^{\prime}}}{\partial a^{j}}=-\beta_{i}^{k^{\prime}} \frac{\partial \xi^{i}}{\partial a^{j}}
$$

O que é realmente imporante é que $\xi^{i}$ e $a^{j}$ são independentes no contexto desta transformação. Neste caso particular, imploca que $\beta_{i}^{k^{\prime}}=\beta_{i}^{k^{\prime}}\left(\xi^{i} ; a^{j}\right)$.

Fazendo uso nos resultados obtidos anteriormente, e das equações (D.5) e (D.9), é possível calcular a variação dos operadores de transformação. Considerando que $f^{i}\left(x^{k^{\prime}}, a^{j}\right)$ foi assumida como $\mathcal{C}^{2}$, a ordem das derivadas parciais é irrelavante (Teorema de Clairaut-Schwartz). Consequentemente, para $\beta_{k^{\prime}}^{i}$, é possível escrever:

$$
\frac{\partial \beta_{k^{\prime}}^{i}}{\partial a^{j}}=\frac{\partial^{2} f^{i}}{\partial a^{j} \partial x^{k^{\prime}}}=\frac{\partial^{2} f^{i}}{\partial x^{k^{\prime}} \partial a^{j}}
$$

Para a transformação inversa, $\beta_{r}^{p^{\prime}}$, usa-se uma conhecida expressão para calcular a derivada de uma matriz inversa (LAX, 2007):

$$
\frac{\partial \beta_{r}^{p^{\prime}}}{\partial a^{j}}=-\beta_{s}^{p^{\prime}}\left(\frac{\partial \beta_{n^{\prime}}}{\partial a^{j}}\right) \beta_{r}^{n^{\prime}}
$$

juntamente com (D.10). Ou, alternativamente, pode-se utilizar a eq. (D.9), para fazer:

$$
\frac{\partial \beta_{r}^{p^{\prime}}}{\partial a^{j}}=\frac{\partial^{2} x^{p^{\prime}}}{\partial a^{j} \partial \xi^{r}}=\frac{\partial^{2} x^{p^{\prime}}}{\partial \xi^{r} \partial a^{j}}=-\frac{\partial}{\partial \xi^{r}}\left(\beta_{n}^{p^{\prime}} \frac{\partial \xi^{n}}{\partial a^{j}}\right)
$$

onde o fato de $x^{k^{\prime}}=g^{k^{\prime}}$ ser $\mathcal{C}^{2}$ foi utilizado.

Alguns fatos devem ser pontuados sobre $f^{i}\left(x^{k^{\prime}} ; a^{j}\right)$ e suas derivadas. Embora essas funções, sozinhas, não são comumente conhecidas dentro do domínio, elas devem ser especificadas nas fronteiras. Na fronteira do corpo essas funções são conhecidas de forma explícita, através da parametrização daquela superfície ao passo que, na fronteira de saída, elas devem ser independentes dos parâmetros de controle $a^{j}$, levando aos resultados das expressões anteriores.

Nesta situação, como deseja-se reduzir as integrais calculadas ao longo do domínio que envolvem variações métricas para integrais calculadas nas fronteiras, 
as expressões (D.9)-(D.12) são suficientes. Elas levarão a formas fechadas das variações nas fronteiras e, por este motivo, expressões que envolvem as derivadas de $f^{i}$ são a base da álgebra apresentada a seguir. Mesmo que ela necessitem ser apresentadas em formas alternativas durante a derivação como, por exemplo, em função de $\xi^{k}, x^{p^{\prime}}$ e $\beta_{k}^{p^{\prime}}$.

\section{D.2 Variação do Jacobiano da Transformação}

Conforme discutido no apêndice A, o Jacobiano da transformação entre os espaços físico e transformado é definido por $J=\operatorname{det}\left(\beta_{k}^{j^{\prime}}\right)$. Uma forma comum de expressar o determinante é fazer o seguinte (FLüGGE, 1972)

$$
J=\frac{1}{6} e^{i r s} e_{j^{\prime} p^{\prime} q^{\prime}} \beta_{i}^{j^{\prime}} \beta_{r}^{p^{\prime}} \beta_{s}^{q^{\prime}}
$$

que é a mesma idéia que está por trás da eq. (A.21).

A primeira variação do Jacobiano resulta em:

$$
\begin{aligned}
\delta J & =\frac{1}{6} e^{i r s} e_{j^{\prime} p^{\prime} q^{\prime}}\left(\delta \beta_{i}^{j^{\prime}} \beta_{r}^{p^{\prime}} \beta_{s}^{q^{\prime}}+\beta_{i}^{j^{\prime}} \delta \beta_{r}^{p^{\prime}} \beta_{s}^{q^{\prime}}+\beta_{i}^{j^{\prime}} \beta_{r}^{p^{\prime}} \delta \beta_{s}^{q^{\prime}}\right) \\
& =\frac{1}{2} e^{i r s} e_{j^{\prime} p^{\prime} q^{\prime}} \delta \beta_{i}^{j^{\prime}} \beta_{r}^{p^{\prime}} \beta_{s}^{q^{\prime}}
\end{aligned}
$$

que é o resultado depois de uma troca dos indíces contraídos. A expressão acima pode ser simplificada utilizando a equação (D.12) para substituir $\delta \beta_{i}^{j^{\prime}}$, e dividindoa por $\delta a^{k}$. Isso resulta em:

$$
\frac{\partial J}{\partial a^{k}}=\frac{1}{2} e^{i r s} e_{j^{\prime} p^{\prime} q^{\prime}} \frac{\partial \beta_{i}^{j^{\prime}}}{\partial a^{k}} \beta_{r}^{p^{\prime}} \beta_{s}^{q^{\prime}}=-\frac{1}{2} e^{i r s} e_{j^{\prime} p^{\prime} q^{\prime}} \frac{\partial}{\partial \xi^{i}}\left(\beta_{n}^{j^{\prime}} \frac{\partial \xi^{n}}{\partial a^{k}}\right) \beta_{r}^{p^{\prime}} \beta_{s}^{q^{\prime}}
$$

Realizando permutações pares dos índices na equação (A.12), pode-se escrever:

$$
J \beta_{j^{\prime}}^{i}=\frac{1}{2} e^{i r s} e_{j^{\prime} p^{\prime} q^{\prime}} \beta_{r}^{p^{\prime}} \beta_{s}^{q^{\prime}}
$$

que pode, então, ser combinado com a eq. (D.15) para obter:

$$
\frac{\partial J}{\partial a^{k}}=-J \beta_{j^{\prime}}^{i} \frac{\partial}{\partial \xi^{i}}\left(\beta_{n}^{j^{\prime}} \frac{\partial \xi^{n}}{\partial a^{k}}\right)=-\frac{\partial}{\partial \xi^{i}}\left(J \frac{\partial \xi^{i}}{\partial a^{k}}\right)
$$

onde foi utilizada a eq. (A.13). 


\section{D.3 Variação do Produto $\boldsymbol{J} \boldsymbol{\beta}_{\boldsymbol{j}^{\prime}}^{i}$}

Além do Jacobiano, o outro termo de variação métrica que aparece explicitamente nas integrais calculadas ao longo do domínio do gradiente adjunto (5.10) é $J \beta_{j^{\prime}}^{i}$. Embora não possua uma nomenclatura específica, esse termo é tão importante no procedimento que está sendo descrito que recebe uma notação especial em (JAMESON; KIM, 2003a),

$$
S_{j^{\prime}}^{i} \equiv J \beta_{j^{\prime}}^{i}=\frac{1}{2} e^{i r s} e_{j^{\prime} p^{\prime} q^{\prime}} \beta_{r}^{p^{\prime}} \beta_{s}^{q^{\prime}} \Rightarrow \frac{\partial S_{j^{\prime}}^{i}}{\partial \xi^{i}}=\left(S_{j^{\prime}}^{i}\right)_{, i}=0
$$

onde foi utilizada a eq. (D.16), e a eq. (A.13) é utilizada em termos de $S_{j^{\prime}}^{i}$ para enfatizar uma propriedade crucial desse novo tensor.

A primeira variação de $S_{j^{\prime}}^{i}$ pode ser derivada diretamente de (D.18). Fazendose duas permutações ímpares com os índices dos símbolos de permutação, e depois trocando apropriadamente os índices sub e superscritos contraídos, chega-se a

$$
\begin{aligned}
\delta S_{j^{\prime}}^{i} & =\frac{1}{2} e^{i r s} e_{j^{\prime} p^{\prime} q^{\prime}} \delta \beta_{r}^{p^{\prime}} \beta_{s}^{q^{\prime}}+\frac{1}{2} e^{i r s} e_{j^{\prime} p^{\prime} q^{\prime}} \beta_{r}^{p^{\prime}} \delta \beta_{s}^{q^{\prime}} \\
& =e^{i r s} e_{j^{\prime} p^{\prime} q^{\prime}} \delta \beta_{r}^{p^{\prime}} \beta_{s}^{q^{\prime}} \\
\frac{\partial S_{j^{\prime}}^{i}}{\partial a^{k}} & =e^{i r s} e_{j^{\prime} p^{\prime} q^{\prime}} \frac{\partial \beta_{r}^{p^{\prime}}}{\partial a^{k}} \beta_{s}^{q^{\prime}}=-e^{i r s} e_{j^{\prime} p^{\prime} q^{\prime}} \frac{\partial}{\partial \xi^{r}}\left(\beta_{n}^{p^{\prime}} \frac{\partial \xi^{n}}{\partial a^{k}}\right) \beta_{s}^{q^{\prime}}
\end{aligned}
$$

onde o último termo do RHS é um resultado direto da eq. (D.12). A regra da diferenciação do produto permite escrever:

$$
\begin{aligned}
\frac{\partial S_{j^{\prime}}^{i}}{\partial a^{k}} & =-e^{i r s} e_{j^{\prime} p^{\prime} q^{\prime}}\left[\frac{\partial}{\partial \xi^{r}}\left(\beta_{n}^{p^{\prime}} \frac{\partial \xi^{n}}{\partial a^{k}} \beta_{s}^{q^{\prime}}\right)-\beta_{n}^{p^{\prime}} \frac{\partial \xi^{n}}{\partial a^{k}} \frac{\partial^{2} x^{q^{\prime}}}{\partial \xi^{r} \partial \xi^{s}}\right] \\
& =-e^{i r s} e_{j^{\prime} p^{\prime} q^{\prime}} \frac{\partial}{\partial \xi^{r}}\left(\beta_{n}^{p^{\prime}} \beta_{s}^{q^{\prime}} \frac{\partial \xi^{n}}{\partial a^{k}}\right)=-\frac{\partial}{\partial \xi^{r}}\left(e^{i r s} e_{j^{\prime} p^{\prime} q^{\prime}} \beta_{n}^{p^{\prime}} \beta_{s}^{q^{\prime}} \frac{\partial \xi^{n}}{\partial a^{k}}\right)(\mathrm{D} .20)
\end{aligned}
$$

O segundo termo do RHS da primeira equação é eliminado por dupla contração com o símbolo de permutação antisimétrico. E a última equação destaca o fato de que, diferentemente de tensores, símbolos de permutação não estão associados a nenhum sistema de coordenadas (A.11).

Agora, a mesma definição (A.11) implica que $e^{i r s}=J \varepsilon^{i r s}$, enquanto o sistema cartesiano fornece $e_{j^{\prime} p^{\prime} q^{\prime}}=\varepsilon_{j^{\prime} p^{\prime} q^{\prime}}$. Substituindo esses tensores pelos termos 
respectivos em (D.20), chega-se a:

$$
\begin{aligned}
\frac{\partial S_{j^{\prime}}^{i}}{\partial a^{k}} & =-\frac{\partial}{\partial \xi^{r}}\left(J \varepsilon^{i r s} \varepsilon_{j^{\prime} p^{\prime} q^{\prime}} \beta_{n}^{p^{\prime}} \beta_{s}^{q^{\prime}} \frac{\partial \xi^{n}}{\partial a^{k}}\right) \\
& =-\frac{\partial}{\partial \xi^{r}}\left(\varepsilon^{i r s} \varepsilon_{m n s} J \beta_{j^{\prime}}^{m} \frac{\partial \xi^{n}}{\partial a^{k}}\right) \\
& =-\frac{\partial}{\partial \xi^{r}}\left[\left(\delta_{m}^{i} \delta_{n}^{r}-\delta_{n}^{i} \delta_{m}^{r}\right) S_{j^{\prime}}^{m} \frac{\partial \xi^{n}}{\partial a^{k}}\right] \\
\frac{\partial S_{j^{\prime}}^{i}}{\partial a^{k}} & =-\frac{\partial}{\partial \xi^{r}}\left(S_{j^{\prime}}^{i} \frac{\partial \xi^{r}}{\partial a^{k}}-S_{j^{\prime}}^{r} \frac{\partial \xi^{i}}{\partial a^{k}}\right)
\end{aligned}
$$

onde foi feito uso do conhecido resultado para uma contração simples entre tensores de permutação (FLüGGE, 1972).

\section{D.4 Definição de Pseudo-Variações Físicas}

O cerne da derivação que é aqui apresentado é baseado nas equações (D.5), (D.9), (D.12), (D.17) e (D.21). O ponto de partida é a equação já mencionada (5.10), aqui reproduzida novamente por conveniência.

$$
\begin{aligned}
\tilde{T}\langle\tilde{\alpha}, \delta \tilde{\mathbf{a}}\rangle= & \int_{0} \oint_{B} \tilde{G} \delta\left|\frac{d S^{\prime}}{d S}\right| \mathrm{d} \tilde{S} \mathrm{~d} \tilde{t}+\int_{\tilde{\Omega}}\left(\frac{\tilde{\Theta}}{J}-\tilde{\varphi}_{i} \tilde{u}^{i}\right)\left[\delta\left(J \beta_{q^{\prime}}^{k}\right) \tilde{u}^{q^{\prime}}\right]_{, k} \mathrm{~d} \tilde{\Omega}+ \\
- & \int_{\tilde{\Omega}} \frac{\tilde{\varphi}_{r^{\prime}}}{J}\left\{\partial_{o}\left(\delta J \tilde{u}^{r^{\prime}}\right)+\left[\delta ( J \beta _ { n ^ { \prime } } ^ { j } ) \left(\tilde{u}^{r^{\prime}} \tilde{u}^{n^{\prime}}+g^{r^{\prime} n^{\prime}} \tilde{p}+\right.\right.\right. \\
& \left.\left.\left.-\frac{1}{R e}\left(\tilde{u}_{, p^{\prime}}^{r^{\prime}} g^{r^{\prime} n^{\prime}}+\tilde{u}_{,{ }^{\prime}}^{p^{\prime}} g^{p^{\prime} n^{\prime}}\right)\right)\right]_{, j}\right\} \mathrm{d} \tilde{\Omega} \\
& +\int_{0} \oint_{\tilde{S}_{i}}\left[-\frac{1}{R e}\left(\left.\tilde{\varphi}_{i}\right|_{j}+\left.\tilde{\varphi}_{j}\right|_{i}\right) n^{j}+\tilde{\Theta} n_{i}\right] \frac{\partial \tilde{f}^{i}}{\partial \tilde{\mathbf{a}}^{k}} \delta \tilde{\mathbf{a}}^{k} \mathrm{~d} \tilde{S} \mathrm{~d} \tilde{t}
\end{aligned}
$$

onde o termo $\tilde{u}_{,{ }^{\prime}}^{p^{\prime}} g^{p^{\prime} n^{\prime}}$ foi acrescentado ao integrando da terceira integral para que a derivação seja feita com a forma completa da equação. A última integral desaparece na ausência de variações não-geométricas. Assim, dividindo-se os termos restantes por $\delta \mathbf{a}^{i}$ - para um $i$ fixo e específico - e tomando o limte $\delta \mathbf{a}^{i} \rightarrow 0$ 
na expressão resultante, obtem-se:

$$
\begin{aligned}
& \tilde{T} \frac{\partial\langle\tilde{\alpha}, \delta \tilde{\mathbf{a}}\rangle}{\partial a^{i}}= \int_{0} \oint_{B} \tilde{G} \frac{\partial}{\partial a^{i}}\left|\frac{d S^{\prime}}{d S}\right| \mathrm{d} \tilde{S} \mathrm{~d} \tilde{t}+\int_{\tilde{\Omega}}\left(\frac{\tilde{\Theta}}{J}-\tilde{\varphi}_{i} \tilde{u}^{i}\right)\left[\frac{\partial S_{q^{\prime}}^{k}}{\partial a^{i}} \tilde{u}^{q^{\prime}}\right]_{, k} \mathrm{~d} \tilde{\Omega}+ \\
&- \int_{\tilde{\Omega}} \frac{\tilde{\varphi}_{r^{\prime}}}{J}\left\{\partial_{o}\left(\frac{\partial J}{\partial a^{i}} \tilde{u}^{r^{\prime}}\right)+\left[\frac { \partial S _ { n ^ { \prime } } ^ { j } } { \partial a ^ { i } } \left(\tilde{u}^{r^{\prime}} \tilde{u}^{n^{\prime}}+g^{r^{\prime} n^{\prime}} \tilde{p}+\right.\right.\right. \\
&\left.\left.\left.-\frac{1}{R e}\left(\tilde{u}_{, p^{\prime}}^{r^{\prime}} g^{r^{\prime} n^{\prime}}+\tilde{u}_{,{ }^{\prime}}^{p^{\prime}} g^{p^{\prime} n^{\prime}}\right)\right)\right]_{, j}\right\} \mathrm{d} \tilde{\Omega}
\end{aligned}
$$

onde a variação da primeira integral pode ser simplesmente derivada da eq. (B.4) ou da eq. (B.7), dependendo da dimensionalidade do escoamento. Como as duas últimas integrais na equação acima são objeto desse apêndice, elas serão tratadas separadamente.

Inicialmente, será tratada a integral em (D.23), aqui denominada $\Delta_{1}$. Para melhor visualização e exibição das equações, as variáveis adjuntas estão agrupadas da seguinte forma $\varpi \equiv\left(\tilde{\Theta} / J-\tilde{\varphi}_{i} \tilde{u}^{i}\right)$. E o parâmetro de derivação nesta integral é substituído pela eq. (D.21), para obter:

$$
\begin{aligned}
\Delta_{1} & =\int_{\tilde{\Omega}} \varpi\left[\frac{\partial S_{q^{\prime}}^{k}}{\partial a^{i}} \tilde{u}^{q^{\prime}}\right]_{, k} \mathrm{~d} \tilde{\Omega}=-\int_{\tilde{\Omega}} \varpi\left[\left(S_{q^{\prime}}^{k} \frac{\partial \xi^{r}}{\partial a^{i}}-S_{q^{\prime}}^{r} \frac{\partial \xi^{k}}{\partial a^{i}}\right)_{, r} \tilde{u}^{q^{\prime}}\right]_{, k} \mathrm{~d} \tilde{\Omega} \\
& \left.=-\int_{\tilde{\Omega}} \varpi\left\{\left[S_{q^{\prime}}^{k} \frac{\partial \xi^{r}}{\partial a^{i}}\right)_{, r} \tilde{u}^{q^{\prime}}\right]_{, k}-\left[S_{q^{\prime}}^{r}\left(\frac{\partial \xi^{k}}{\partial a^{i}}\right)_{, r} \tilde{u}^{q^{\prime}}\right]_{, k}\right\}_{\mathrm{\Omega}} \mathrm{d} \tilde{\Omega} \\
& =-\int_{\tilde{\Omega}} \varpi\left\{\left[\left(J \tilde{u}^{k} \frac{\partial \xi^{r}}{\partial a^{i}}\right)_{, r}-\tilde{u}_{, r}^{q^{\prime}} S_{q^{\prime}}^{k} \frac{\partial \xi^{r}}{\partial a^{i}}-\left(J \tilde{u}^{r} \frac{\partial \xi^{k}}{\partial a^{i}}\right)_{, r}+\frac{\partial \xi^{k}}{\partial a^{i}}\left(J \tilde{u}^{r}\right)_{, r}\right]_{, k}\right\} \mathrm{d} \tilde{\Omega} \\
& \left.=-\int_{\tilde{\Omega}} \varpi\left\{\left(J \tilde{u}^{k} \frac{\partial \xi^{r}}{\partial a^{i}}\right)_{, r k}-\left(\tilde{u}_{, r}^{q^{\prime}} S_{q^{\prime}}^{k} \frac{\partial \xi^{r}}{\partial a^{i}}\right)_{, k}-\left(J \tilde{u}^{r} \frac{\partial \xi^{k}}{\partial a^{i}}\right)_{, r k}\right\} \mathrm{d} \tilde{\Omega}{ }_{, i} \mathrm{D} .24\right) \\
& =\int_{\tilde{\Omega}} \varpi\left(\tilde{u}_{, r}^{q^{\prime}} S_{q^{\prime}}^{k} \frac{\partial \xi^{r}}{\partial a^{i}}\right)_{, k} \mathrm{~d} \tilde{\Omega}
\end{aligned}
$$

onde a eq. (D.18) foi utilizada entre as primeira e segunda linhas. A equação da continuuidade em (A.40) implica que o último termo na integral da terceira linha desaparece. Na quarta linha, o caráter $\mathcal{C}^{2}$ de todas as variações métricas permite que a ordem das derivadas parciais seja trocada, juntamente com os índices contraidos dos primeiro e terceiro termos. Como resultado disso, os termos se cancelam e haverá somente um termo restante na quinta linha. Este termo, 
por sua vez, pode ser tratado da seguinte forma:

$$
\Delta_{1}=\int_{\tilde{\Omega}} \varpi J \beta_{q^{\prime}}^{k} \frac{\partial}{\partial \xi^{k}}\left(\frac{\partial \tilde{u}^{q^{\prime}}}{\partial \xi^{r}} \frac{\partial \xi^{r}}{\partial a^{i}}\right)=\int_{\tilde{\Omega}} J \varpi \frac{\partial}{\partial x^{q^{\prime}}}\left(\frac{\partial \tilde{u}^{q^{\prime}}}{\partial \xi^{r}} \frac{\partial \xi^{r}}{\partial a^{i}}\right) \mathrm{d} \tilde{\Omega}
$$

Aqui, novamente, utilizou-se a eq. (D.18), e a regra da cadeia.

A pseudo-variação física do vetor velocidade é definido baseando-se na equação acima:

$$
\frac{\partial \check{u}^{q^{\prime}}}{\partial a^{i}} \equiv \frac{\partial \tilde{u}^{q^{\prime}}}{\partial \xi^{r}} \frac{\partial \xi^{r}}{\partial a^{i}}=\beta_{r}^{s^{\prime}} \frac{\partial \tilde{u}^{q^{\prime}}}{\partial x^{s^{\prime}}}\left(-\beta_{k^{\prime}}^{r} \frac{\partial x^{k^{\prime}}}{\partial a^{i}}\right)=-\frac{\partial \tilde{u}^{q^{\prime}}}{\partial x^{k^{\prime}}} \frac{\partial x^{k^{\prime}}}{\partial a^{i}}
$$

onde se utilizou a regra da cadeia e a eq. (D.9). Assim, substituindo essa definição pelo termo correspondente na eq. (D.25) obtem-se:

$$
\Delta_{1}=\int_{\tilde{\Omega}}\left(\tilde{\Theta}-J \tilde{\varphi}_{i} \tilde{u}^{i}\right)\left(\frac{\partial \check{u}^{q^{\prime}}}{\partial a^{i}}\right)_{, q^{\prime}} \mathrm{d} \tilde{\Omega}=\int_{\tilde{\Omega}}(\tilde{\Theta}-J \tilde{\varphi} \cdot \tilde{\mathbf{u}}) \nabla \cdot\left(\frac{\partial \check{\mathbf{u}}}{\partial a^{i}}\right) \mathrm{d} \tilde{\Omega}
$$

que é a forma final da integral $\Delta_{1}$. É importante notar aqui a similaridade dessa integral com o funcional $I_{2}$ da eq. (3.37).

Agora, será tratada a terceira integral de (D.23), aqui denominada $\Delta_{2}$ :

$$
\begin{array}{r}
\Delta_{2}=-\int_{\tilde{\Omega}} \frac{\tilde{\varphi}_{r^{\prime}}}{J}\left\{\partial_{o}\left(\frac{\partial J}{\partial a^{i}} \tilde{u}^{r^{\prime}}\right)+\left[\frac { \partial S _ { n ^ { \prime } } ^ { j } } { \partial a ^ { i } } \left(\tilde{u}^{r^{\prime}} \tilde{u}^{n^{\prime}}+g^{r^{\prime} n^{\prime}} \tilde{p}+\right.\right.\right. \\
\left.\left.\left.-\frac{1}{R e}\left(\tilde{u}_{, p^{\prime}}^{r^{\prime}} g^{p^{\prime} n^{\prime}}+\tilde{u}_{,{ }^{\prime}}^{p^{\prime}} g^{r^{\prime} n^{\prime}}\right)\right)\right]_{, j}\right\} \mathrm{d} \tilde{\Omega}
\end{array}
$$

Para facilitar a álgebra, define-se o tensor cartesiano de segunda ordem

$$
\begin{aligned}
A^{r^{\prime} n^{\prime}} \equiv \tilde{u}^{r^{\prime}} \tilde{u}^{n^{\prime}}+g^{r^{\prime} n^{\prime}} \tilde{p}-\frac{1}{R e}\left(\tilde{u}_{,{ }^{\prime}}^{r^{\prime}} g^{k^{\prime} n^{\prime}}+\tilde{u}_{, r^{\prime}}^{k^{\prime}} g^{r^{\prime} n^{\prime}}\right) \\
\Rightarrow \partial_{o}\left(J \tilde{u}^{r^{\prime}}\right)=-\left(S_{n^{\prime}}^{j} A^{r^{\prime} n^{\prime}}\right)_{, j}=-\left(J A^{r^{\prime} j}\right)_{, j}
\end{aligned}
$$

Aqui, a equação de momento de (A.40) é escrita em termos de $A^{r^{\prime} n^{\prime}}$, na direita. Com essa definição, juntamente com as eqs. (D.17) e (D.21), pode-se escrever $\Delta_{2}$ na seguinte forma:

$$
\begin{aligned}
\Delta_{2} & =\int_{\tilde{\Omega}} \frac{\tilde{\varphi}_{r^{\prime}}}{J}\left\{\partial_{o}\left[\left(J \frac{\partial \xi^{k}}{\partial a^{i}}\right)_{, k} \tilde{u}^{r^{\prime}}\right]+\left[\left(S_{n^{\prime}}^{j} \frac{\partial \xi^{p}}{\partial a^{i}}-S_{n^{\prime}}^{p} \frac{\partial \xi^{j}}{\partial a^{i}}\right)_{, p} A^{r^{\prime} n^{\prime}}\right]_{, j}\right\} \mathrm{d} \tilde{\Omega} \\
& =\int_{\tilde{\Omega}} \frac{\tilde{\varphi}_{r^{\prime}}}{J}\left\{\left(J \frac{\partial \xi^{k}}{\partial a^{i}}\right)_{, k} \partial_{o} \tilde{u}^{r^{\prime}}+\left[\left(S_{n^{\prime}}^{j} \frac{\partial \xi^{p}}{\partial a^{i}}\right)_{, p} A^{r^{\prime} n^{\prime}}-S_{n^{\prime}}^{p}\left(\frac{\partial \xi^{j}}{\partial a^{i}}\right)_{, p} A^{r^{\prime} n^{\prime}}\right]_{, j}\right\} \mathrm{d} \tilde{\Omega}
\end{aligned}
$$


onde foi assumido que nem os termos relativos à métrica nem suas respectivas variações são tempo dependentes - embora a física do escoamento possa sê-lo. Utilizou-se também a eq. (D.18), e a expressão resultante pode ser manipulada da seguinte maneira:

$$
\begin{aligned}
\Delta_{2}= & \int_{\tilde{\Omega}} \frac{\tilde{\varphi}_{r^{\prime}}}{J}\left\{\left(J \frac{\partial \xi^{k}}{\partial a^{i}}\right)_{, k} \partial_{o} \tilde{u}^{r^{\prime}}+\left[\left(S_{n^{\prime}}^{j} \frac{\partial \xi^{p}}{\partial a^{i}}\right)_{, p} A^{r^{\prime} n^{\prime}}-\left(\frac{\partial \xi^{j}}{\partial a^{i}}\right)_{, p} J A^{r^{\prime} p}\right]_{, j}\right\} \mathrm{d} \tilde{\Omega} \\
= & \int_{\tilde{\Omega}} \frac{\tilde{\varphi}_{r^{\prime}}}{J}\left\{\left(J \frac{\partial \xi^{k}}{\partial a^{i}} \partial_{o} \tilde{u}^{r^{\prime}}\right)_{, k}-J \partial_{o} \frac{\partial \check{u}^{r^{\prime}}}{\partial a^{i}}+\left[\left(S_{n^{\prime}}^{j} \frac{\partial \xi^{p}}{\partial a^{i}}\right)_{, p} A^{r^{\prime} n^{\prime}}-\left(\frac{\partial \xi^{j}}{\partial a^{i}}\right)_{, p} J A^{r^{\prime} p}\right]_{, j}\right\} \mathrm{d} \tilde{\Omega} \\
= & \int_{\tilde{\Omega}} \frac{\tilde{\varphi}_{r^{\prime}}}{J}\left\{\left(J \frac{\partial \xi^{k}}{\partial a^{i}} \partial_{o} \tilde{u}^{r^{\prime}}\right)_{, k}-J \partial_{o} \frac{\partial \check{u}^{r^{\prime}}}{\partial a^{i}}+\left(J A^{r^{\prime} j} \frac{\partial \xi^{p}}{\partial a^{i}}\right)_{, p j}-\left[S_{n^{\prime}}^{j} \frac{\partial \xi^{p}}{\partial a^{i}}\left(A^{r^{\prime} n^{\prime}}\right)_{, p}\right]_{, j}+\right. \\
& \left.\left.-\left(\frac{\partial \xi^{j}}{\partial a^{i}} J A^{r^{\prime} p}\right)_{, p j}+\left[\frac{\partial \xi^{j}}{\partial a^{i}}\left(J A^{r^{\prime} p}\right)_{, p}\right]_{, j}\right\} \mathrm{~d} \tilde{\Omega} .31\right)
\end{aligned}
$$

A definição da pseudo-variação da velocidade foi introduzida na segunda linha. O terceito termo na terceira linha e o primeiro na quarta linha se cancelam, uma vez que a ordem das derivadas parciais é irrelevante. Ademais, o último termo na quarta linha pode ser substituído pelo LHS da eq. (D.29),

$$
\begin{gathered}
\Delta_{2}=\int_{\tilde{\Omega}} \frac{\tilde{\varphi}_{r^{\prime}}}{J}\left\{\left(J \frac{\partial \xi^{k}}{\partial a^{i}} \partial_{o} \tilde{u}^{r^{\prime}}\right)_{, k}-J \partial_{o} \frac{\partial \check{u}^{r^{\prime}}}{\partial a^{i}}-\left[S_{n^{\prime}}^{j} \frac{\partial \xi^{p}}{\partial a^{i}}\left(A^{r^{\prime} n^{\prime}}\right)_{, p}\right]_{, j}+\right. \\
\left.-\left(\frac{\partial \xi^{j}}{\partial a^{i}} J \partial_{o} \tilde{u}^{r^{\prime}}\right)_{, j}\right\} \mathrm{d} \tilde{\Omega} \\
\Delta_{2}=-\int_{\tilde{\Omega}} \frac{\tilde{\varphi}_{r^{\prime}}}{J}\left\{J \partial_{o} \frac{\partial \check{u}^{r^{\prime}}}{\partial a^{i}}+\left[S_{n^{\prime}}^{j} \frac{\partial \xi^{p}}{\partial a^{i}}\left(A^{r^{\prime} n^{\prime}}\right)_{, p}\right]_{, j}\right\} \mathrm{d} \tilde{\Omega}
\end{gathered}
$$

Aqui, utilizou-se o fato do termo relativo à métrica $J$ não ser tempo-dependente, o que permite que os primeiro e quarto termos dentro dos parênteses sejam cancelados.

Agora, a definição de $A^{r^{\prime} n^{\prime}}$ em (D.29) pode substituir os termos correspondentes na equação acima (D.32), para obter:

$$
\begin{aligned}
\Delta_{2}=-\int_{\tilde{\Omega}} \frac{\tilde{\varphi}_{r^{\prime}}}{J}\left\{J \partial_{o} \frac{\partial \check{u}^{r^{\prime}}}{\partial a^{i}}\right. & {\left[S _ { n ^ { \prime } } ^ { j } \frac { \partial \xi ^ { p } } { \partial a ^ { i } } \left(\tilde{u}^{r^{\prime}} \tilde{u}^{n^{\prime}}+g^{r^{\prime} n^{\prime}} \tilde{p}+\right.\right.} \\
& \left.\left.\left.-\frac{1}{R e}\left(\tilde{u}_{, k^{\prime}}^{r^{\prime}} g^{k^{\prime} n^{\prime}}+\tilde{u}_{, r^{\prime}}^{k^{\prime}} g^{r^{\prime} n^{\prime}}\right)\right)_{, p}\right]_{, j}\right\} \mathrm{d} \tilde{\Omega}
\end{aligned}
$$

e é bom frisar que o tensor métrico cartesiano $g^{p^{\prime} n^{\prime}}$ é uma matriz identidade. 
Maiores detalhes do tratamento do termo difusivo estão disponíveis em (VOLPE, 2011). Consequentemente, a equação acima passa a ser:

$$
\begin{gathered}
\Delta_{2}=-\int_{\tilde{\Omega}} \frac{\tilde{\varphi}_{r^{\prime}}}{J}\left\{J \partial_{o} \frac{\partial \check{u}^{r^{\prime}}}{\partial a^{i}}+S_{n^{\prime}}^{j}\left[\frac { \partial \xi ^ { p } } { \partial a ^ { i } } \left(\tilde{u}^{r^{\prime}} \tilde{u}^{n^{\prime}}+g^{r^{\prime} n^{\prime}} \tilde{p}+\right.\right.\right. \\
\left.\left.\left.-\frac{1}{R e}\left(\tilde{u}_{, k^{\prime}}^{r^{\prime}} g^{k^{\prime} n^{\prime}}+\tilde{u}_{,{ }^{\prime}}^{k^{\prime}} g^{r^{\prime} n^{\prime}}\right)\right)_{, p}\right]_{, j}\right\} \mathrm{d} \tilde{\Omega} \\
=-\int_{\tilde{\Omega}} \frac{\tilde{\varphi}_{r^{\prime}}}{J}\left\{J \partial_{o} \frac{\partial \check{u}^{r^{\prime}}}{\partial a^{i}}+S_{n^{\prime}}^{j}\left[\frac{\partial \check{u}^{r^{\prime}}}{\partial a^{i}} \tilde{u}^{n^{\prime}}+\tilde{u}^{r^{\prime}} \frac{\partial \check{u}^{r^{\prime}}}{\partial a^{i}}+g^{r^{\prime} n^{\prime}} \frac{\partial \check{p}}{\partial a^{i}}+\right.\right. \\
\left.\left.-\frac{1}{R e}\left(\left(\frac{\partial \check{u}^{r^{\prime}}}{\partial a^{i}}\right)_{, k^{\prime}} g^{k^{\prime} n^{\prime}}+\left(\frac{\partial \check{u}^{k^{\prime}}}{\partial a^{i}}\right)_{, r^{\prime}} g^{r^{\prime} n^{\prime}}\right)\right]_{, j}\right\} \mathrm{d} \tilde{\Omega}
\end{gathered}
$$

onde a pseudo-variação física da pressão é definida analogamente à da velocidade

$$
\frac{\partial \check{p}}{\partial a^{i}} \equiv \frac{\partial \tilde{p}}{\partial \xi^{p}} \frac{\partial \xi^{p}}{\partial a^{i}}=\beta_{p}^{s^{\prime}} \frac{\partial \tilde{p}}{\partial x^{s^{\prime}}}\left(-\beta_{k^{\prime}}^{p} \frac{\partial x^{k^{\prime}}}{\partial a^{i}}\right)=-\frac{\partial \tilde{p}}{\partial x^{k^{\prime}}} \frac{\partial x^{k^{\prime}}}{\partial a^{i}}
$$

e, novamente, foram utilizadas a regra de cadeia e a eq. (D.9). A equação (D.34) é a forma final da integral $\Delta_{2}$. Aqui, novamente, é importante notar a semelhança entre a integral acima e o funcional $I_{3}$ da eq. (3.37).

Substituindo as eqs. (D.27) e (D.34) por $\Delta_{1}$ e $\Delta_{2}$ em (D.23), a expressão do gradiente geométrico passa a ser:

$$
\begin{array}{r}
\tilde{T} \frac{\partial\langle\tilde{\alpha}, \delta \tilde{\mathbf{a}}\rangle}{\partial a^{i}}= \\
\int_{0} \oint_{B} \tilde{G} \frac{\partial}{\partial a^{i}}\left|\frac{d S^{\prime}}{d S}\right| \mathrm{d} \tilde{S} \mathrm{~d} \tilde{t}+\int_{\tilde{\Omega}}\left(\tilde{\Theta}-J \tilde{\varphi}_{i} \tilde{u}^{i}\right)\left(\frac{\partial \check{u}^{q^{\prime}}}{\partial a^{i}}\right)_{, q^{\prime}} \mathrm{d} \tilde{\Omega}-\int_{\tilde{\Omega}} \frac{\tilde{\varphi}_{r^{\prime}}}{J}\left\{J \frac{\partial^{2} \check{u}^{r^{\prime}}}{\partial t \partial a^{i}}+\right. \\
+S_{n^{\prime}}^{j}\left[\frac{\partial \check{u}^{r^{\prime}}}{\partial a^{i}} \tilde{u}^{n^{\prime}}+\tilde{u}^{r^{\prime}} \frac{\partial \check{u}^{r^{\prime}}}{\partial a^{i}}+g^{r^{\prime} n^{\prime}} \frac{\partial \check{p}}{\partial a^{i}}-\frac{1}{R e}\left(\left(\frac{\partial \check{u}^{r^{\prime}}}{\partial a^{i}}\right)_{, k^{\prime}} g^{k^{\prime} n^{\prime}}+\right.\right. \\
\left.\left.+\left(\frac{\partial \check{u}^{k^{\prime}}}{\partial a^{i}}\right)_{, r^{\prime}} g^{r^{\prime} n^{\prime}}\right)\right]_{, j} \mathrm{~d} \tilde{\Omega}
\end{array}
$$

Agora, o próximo passo é integrar $\Delta_{1}$ e $\Delta_{2}$ por partes, de maneira análoga àquela que foi feita com as integrais correspondentes $I_{2}$ e $I_{3}$, da eq. (3.37). 


\section{D.5 Integração por Partes dos Funcionais do Gra- diente}

Como mencionado, o procedimento é similar ao feito no capítulo 3 e, portanto, as etapas da derivação serão suprimidas para facilitar a leitura. Iniciar-se-á pela integral $\Delta_{1}$, cujo integrando é similar ao da integral $I_{2}$ da eq. (3.37). O resultado da integração por partes é:

$$
\Delta_{1}=\int_{0}^{\tilde{T}} \oint_{B}\left(\tilde{\Theta}-J \tilde{\varphi}_{i} \tilde{u}^{i}\right) \delta \check{u}^{q^{\prime}} n_{q^{\prime}} d S d t-\int_{\tilde{\Omega}}\left(\tilde{\Theta}-J \tilde{\varphi}_{i} \tilde{u}^{i}\right)_{, q^{\prime}} \delta \check{u}^{q^{\prime}} d \tilde{\Omega}
$$

onde definiu-se: $\check{u}^{q^{\prime}} \equiv \frac{\partial \breve{u}^{q^{\prime}}}{\partial a^{p}} d a^{p}$ Observando a integral $\Delta_{2}$ nota-se que o integrando é similar ao da integral $I_{3}$ da eq. (3.37). Fazendo-se o mesmo procedimento para todos os termos de $\Delta_{2}$, agrupando-os juntamente com os demais termos do gradiente e suprimindo o símbolo , vem:

$$
\begin{aligned}
T \delta I= & \int_{0}^{T} \oint_{V}\left\{\left.\varphi^{r^{\prime}}\right|_{r^{\prime}}(\delta p-\delta \check{p})+\left[\partial_{0} \varphi_{r^{\prime}}+\left.u^{n^{\prime}} \varphi_{r^{\prime}}\right|_{n^{\prime}}-\left.\varphi_{n^{\prime}} u^{n^{\prime}}\right|_{r^{\prime}}-\left.\Theta\right|_{r^{\prime}}\right.\right. \\
& \left.\left.+\left.S_{n^{\prime}}^{j} \frac{1}{R e}\left(\left.\varphi_{r^{\prime}}\right|^{n^{\prime}}+\left.\varphi^{n^{\prime}}\right|_{r^{\prime}}\right)\right|_{n^{\prime}}\right] \times\left(\delta u^{i}-\delta \check{u}^{r^{\prime}}\right)\right\} \mathrm{d} V \mathrm{~d} t \\
& -\int_{0}^{T} \oint_{\partial V}\left\{\left[u^{n^{\prime}} \varphi_{r^{\prime}} \mathbf{n}_{\mathbf{n}^{\prime}}-\Theta \mathbf{n}_{\mathbf{r}^{\prime}}+\frac{1}{R e}\left(\left.\varphi_{r^{\prime}}\right|_{n^{\prime}}+\left.\varphi_{n^{\prime}}\right|_{r^{\prime}}\right) \mathbf{n}^{\mathbf{n}^{\prime}}\right]\left(\delta u^{r^{\prime}}-\delta \check{u}^{r^{\prime}}\right)\right. \\
& \left.+\varphi_{r^{\prime}}\left[\left(\delta p \mathbf{n}^{\mathbf{r}^{\prime}}-\delta \mathcal{F}^{r^{\prime}}\right)-\left(\delta \check{p} \mathbf{n}^{\mathbf{i}}-\delta \check{\mathcal{F}}^{r^{\prime}}\right)\right]\right\} \mathrm{d} S \mathrm{~d} t \\
& \left.+\oint_{V} \varphi_{r^{\prime}}\left(\delta u^{i}-\delta \check{u}^{r^{\prime}}\right) \mathrm{d} V\right]_{0}^{T}+\int_{0}^{T} \oint_{\partial V} G \delta\left|\frac{d S^{\prime}}{d S}\right| \mathrm{d} S \mathrm{~d} t \\
& \int_{0}^{T} \oint_{\partial V}\left[\frac{\partial G}{\partial p} \delta p+\frac{\partial G}{\partial \mathcal{F} k} \delta \mathcal{F}^{k}\right]\left|\frac{d S^{\prime}}{d S}\right| \mathrm{d} S \mathrm{~d} t
\end{aligned}
$$

A primeira integral, como é possível observar, contém as equações adjuntas exibidas na eq. (3.46). Quando essas equações são satisfeitas, a primeira integral da expressão do gradiente se anula. A quarta integrais permanece da forma que foi apresentada enquanto as segunda, terceira e quinta integrais possuem termos que se anulam com a definição do problema de contorno adjunto, como definido no capítulo 3 na seção 3.2.3. Mais especificamente, os termos que multiplicam a combinação das variações físicas e pseudo-variações, se anulam nas condições de entrada, saída e planos de simetria. A condição de parede faz com que somente a quinta integral se anule mas não anula todos os termos da segunda integral. $\mathrm{Na}$ verdade somente o primeiro termo da terceira integral é nulo na parede enquanto os demais não são. Assim, restarão apenas os termos que multiplicam as 
pseudo-variações visto que variações físicas se anulam nessas fronteiras. Por fim, as condições temporais fazem com que a terceira integral seja igual a zero. $\mathrm{Na}$ avaliação das integrais restantes considera-se que as pseudo variações se anulam em fronteiras fixas. Isso está discutido mais detalhadamente na conclusão deste apêndice, apresentada a seguir. A equação final do gradiente reduzido é dada por:

$$
\begin{aligned}
T \delta I=\int_{0}^{T} \oint_{\partial V}\left\{\left[-\Theta \mathbf{n}_{\mathbf{r}^{\prime}}\right.\right. & \left.+\frac{1}{R e}\left(\left.\varphi_{r^{\prime}}\right|_{n^{\prime}}+\varphi_{n^{\prime}} \mid r^{\prime}\right) \mathbf{n}^{\mathbf{n}^{\prime}}\right] \delta \check{u}^{r^{\prime}} \\
& \left.+\varphi_{r^{\prime}}\left[\left(\delta p \mathbf{n}^{\mathbf{r}^{\prime}}-\delta \mathcal{F}^{r^{\prime}}\right)-\left(\frac{\partial \check{p}}{\partial a_{i}} \mathbf{n}^{\mathbf{i}}-\delta \check{\mathcal{F}}^{r^{\prime}}\right)\right]\right\} \mathrm{d} S \mathrm{~d} t+ \\
& +\int_{0}^{T} \oint_{\partial V} G \delta\left|\frac{d S^{\prime}}{d S}\right| \mathrm{d} S \mathrm{~d} t
\end{aligned}
$$

\section{D.6 Conclusões}

Ao final da seção D.1, foi mencionado que esperava-se que as fronteiras ao longe do escoamento fossem independentes dos parâmetros de controle $a^{i}$, que controlam a geometria do corpo: $\xi^{i}=f^{i}\left(x^{k^{\prime}}, a^{j}\right)$. De fato, isso deve ocorrer com qualquer superfície fixa dentro do domínio - $\xi^{i}=\xi^{i}\left(x^{k^{\prime}}\right)$ - seja uma fronteira de entrada, saída ou até um plano de simetria. Essas fronteiras independem dos parâmetros de controle e, consequentemente, a eq. (D.5) deve resultar, em cada uma elas, em $\partial \xi^{i} / \partial a^{k}=0$. Embora essas derivadas possam ser não nulas em uma vizinhança dessas fronteiras, o caráter $\mathcal{C}^{2}$ assumido para a transformação $\xi^{i}=f^{i}\left(x^{k^{\prime}}, a^{j}\right)$ garante que elas se anulem suavemente.

Um resultado imediato disso é que as derivadas paramétricas nas eqs. (D.9), (D.10), (D.12), (D.17) e (D.21) devem ser nulas em toda superfície fixa. Por outro lado, significa que as pseudo-variações físicas da velocidade (D.26) e pressão (D.35) devem ir a zero ali. Isso implica, ainda, que a variação geométrica das equações governantes do escoamento (3.36) devem desaparecer nessas superfícies, embora possam ser não nulas nas suas respectivas vizinhanças. Isso possui impacto direto na derivação das condições de contorno adjuntas. 